\title{
WHAT DO PALEO-GEOCHEMICAL TRACERS TELL US ABOUT THE DEEP OCEAN CIRCULATION DURING THE

LAST ICE AGE?

by

\section{Pascal LeGrand}

M.S. Ecole Centrale Paris (1990)

Submitted in partial fulfillment of the requirements for the degree of Doctor of Philosophy

at the

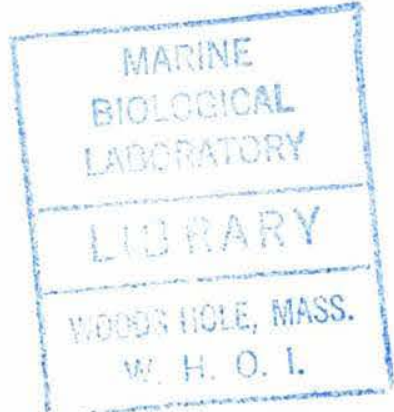
MASSACHUSETTS INSTITUTE OF TECHNOLOGY
and the

WOODS HOLE OCEANOGRAPHIC INSTITUTION

September 1994

(C) Pascal LeGrand 1994

The author hereby grants to MIT and to WHOI permission to reproduce and to distribute copies of this thesis document in whole or in part.

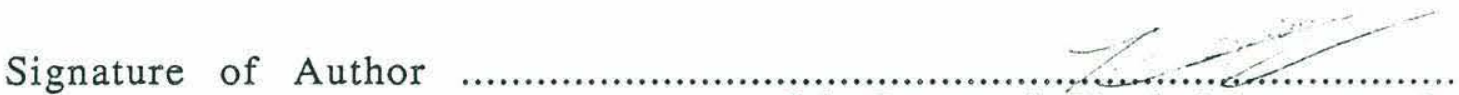
Joint Program in Physical Oceanography Massachusetts Institute of Technology Woods Hole Qceanographic Institution

Certified by

May 23, 1994

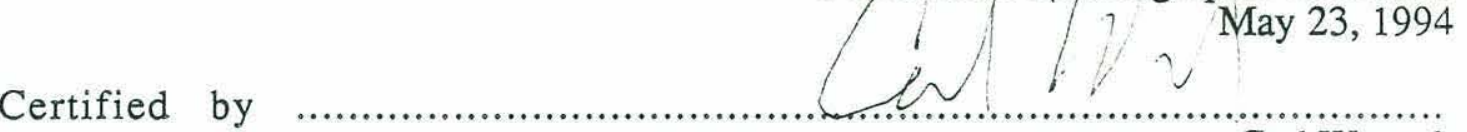

Carl Wunsch

Cecil and Ida Green Professor of Physical Oceanography

Accepted by

Thesis Supervisor

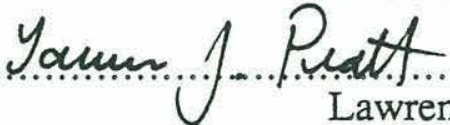

Lawrence J. Pratt

Chairman, Joint Committee for Physical Oceanography

Massachusetts Institute of Technology

Woods Hole Oceanographic Institution

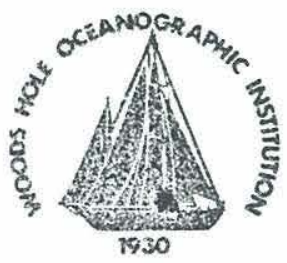




\begin{abstract}
:
Paleo-tracers such as carbon 13 and cadmium show that the deep Atlantic was enriched in nutrients during the Last Ice Age. The conventionally accepted interpretation of these higher nutrient levels is that a reduction of the rate of formation of nutrient-depleted Lower North Atlantic Deep Water (Lower NADW) allowed nutrient-rich Antarctic Bottom Water (AABW) to push further north during the Last Glacial Maximum (LGM) (Boyle and Keigwin, 1982; 1987; Duplessy et al., 1988). The evidence for this interpretation is re-examined in this work, with an emphasis on the quantitative analysis of the paleo-data.

An end-member analysis of the $\delta^{13} \mathrm{C}$ data indicates a larger volume of $\mathrm{AABW}$ and a smaller volume of Lower NADW during the LGM. It is not yet possible, however, to quantify the extent of the volume differences between the modern and the glacial distributions, because the LGM $\delta^{13} \mathrm{C}$ end-members are poorly known.

The second issue examined in this thesis deals with the interpretation of the water mass distribution, inferred from paleo-tracers, in terms of the oceanic circulation. Using a dynamical inverse model of the North Atlantic and a kinematic inverse model of the South Atlantic, it is shown that a tracer distribution corresponding to a significantly reduced volume of Lower NADW does not necessarily correspond to a reduced flux of NADW. Indeed, a circulation almost identical to a modern ocean reference circulation is consistent with the available LGM $\delta^{13} \mathrm{C}$ and $\delta^{18} \mathrm{O}$ data. A flux of Lower NADW reduced by $50 \%$, though not needed to explain the LGM tracer distribution, is also consistent with the data. Thus, the paleo-tracers $\delta^{13} \mathrm{C}$ and $\delta^{18} \mathrm{O}$ do not suffice to quantify the flux of NADW in the glacial ocean. The modern ocean circulation is one of many possible circulations consistent with the available $\delta^{13} \mathrm{C}$ and $\delta^{18} \mathrm{O}$ data.
\end{abstract}




\section{Acknowledgments}

I would first like to thank my thesis advisor, Carl Wunsch, for the guidance and support he provided me throughout this work. One of Carl's greatest qualities is his ability to work in very different fields and to immediately see what the important problems are. I greatly benefited from his experience and his insight, and I hope I will be able to keep the broad view of oceanography he has taught me.

I am indebted to Ed Boyle for his help and patience. I learned most of what I know in paleoceanography from him. I am also grateful to the other members of my thesis committee: John Marshall, Jochem Marotzke, Eric Sundquist, and Bruce Warren for their constant enthusiasm and support.

Thanks are due to Jean-Francois Minster and the people who work at the GRGS Laboratory in Toulouse for hosting me in Spring 1994, and to Pat Lohmann and Lloyd Keigwin who kindly provided me with some pre-published data.

I would never have been able to put up with living at MIT for several years without the constant presence of my friends. I am going to miss them and all the fun time we had together. I am also going to miss Woods Hole in the summer and the "free-food" provided by the Joint program. Emmanuelle gave me the support I needed during my stay in the US, and it was

refreshing to be with someone who thinks that there are things more important in life than getting a Ph.D.

I would like to dedicate this thesis to my father. He did not have the opportunity to stay in school, but I am sure he would have liked to study the way nature works.

This research was funded by the National Science Foundation under grant OCE-9205942. 


\section{Table of contents}

Chapter I - Introduction.

Chapter II - Geochemical evidence for a reduced volume of Lower North Atlantic Deep Water during the Last Glacial

Maximum.

A - The data

1) Deep water geochemical tracers 16

a/ $\delta^{18} \mathrm{O}$, a proxy for global ice volume and temperature: $\quad 16$

b/ Nutrient related tracers 18

$\mathrm{c} / \Delta^{14} \mathrm{C}$, a radioactive tracer $\quad 20$

d/ The conventionally accepted interpretation of deep water paleo-tracers $\quad 21$

2) Surface water 22

3) Availability and quality of oceanic climate indicators 24

4) Land and atmospheric records 26

B - Geochemical cycles in the ocean 26

1) The nutrient cycle 27

2) Preformed nutrients 29

3) Preformed $\delta^{13} \mathrm{C}$

a/ Biological fractionation $\quad 30$

b/ Ocean-Atmosphere exchanges 30

C - Data compilation $\quad 34$

D - Interpretation of the paleo-data in terms of changes in the water $\begin{array}{ll}\text { mass distribution } & 41\end{array}$

1) Quantifying the changes in the water mass distribution 41

2) Discrepancies between $\delta^{13} \mathrm{C}$ data and Cadmium data 54

3) $\delta^{18} \mathrm{O}$ and temperature effects 54

4) Carbon 14 data 56 
Summary $\quad 58$

Chapter III - Combining physical and observational constraints: $\begin{array}{ll}\text { an optimization problem. } & 61\end{array}$

1) Least square objective function 63

2) A practical approach 66

3) Minimax algorithm (for small systems only) 68

4) Local minima 69

Chapter IV - A Coarse resolution inverse model of the North 71 Atlantic.

1) Model domain 71

2) Numerical representation of the physics 75

3) Physical constraints 76

$\begin{array}{ll}\text { a/ Mass conservation } & 76\end{array}$

b/ Potential temperature, salinity, phosphate and ${ }^{13} \mathrm{C}$ conservation $\quad 77$

1) modern ocean $\quad 79$

2) $L G M$ ocean $\quad 79$

c/ Thermal wind $\quad 82$

d/ Linear vorticity balance $\quad 83$

e/ Linear equation of state $\quad 86$

4) Data constraints 89

5) How much imbalance in the physical constraints is acceptable? 94

a/ Mass conservation $\quad 96$

b/ Tracer conservation $\quad 97$

$\begin{array}{ll}\mathrm{c} / \text { Thermal wind balance } & 98\end{array}$

d/ Linear vorticity balance $\quad 98$

6) Problems associated with the non-linear inversion 99

a/ Preventing a no flow solution $\quad 99$

b/ Transports in the Deep Western Boundary Current 101 
$\mathrm{c}$ Local minima of the cost function

102

d/ Sensitivity to weight matrices

104

\section{Chapter V - Changes in the Deep Atlantic circulation during} the Last Glacial Maximum. Results from a quantitative inverse modeling study.

A - Run [0]: A reference solution in the modern ocean 108

B - Last Glacial Maximum Inversions 119

1) Difficulties associated with Last Glacial Maximum simulations: 119

2) Run [1]: smallest changes to the modem ocean circulation necessary to make it consistent with the Last Glacial Maximum $\delta^{18} \mathrm{O}$ and $\delta^{13} \mathrm{C}$ data

3) Run [2]: rate of formation of Lower NADW reduced to half its modern ocean value

4) Are the results of the North Atlantic Model consistent with the South Atlantic data?

a/ Modern ocean simulation

b/ LGM simulation of the South Atlantic with strong NADW formation

c/ LGM simulation of the South Atlantic with reduced Lower NADW formation

C - Discussion

1) Why is it possible to reproduce the LGM tracer distribution without changing much the modem ocean circulation?

2) Why is it possible to find several oceanic circulations compatible with the LGM $\delta^{13} \mathrm{C}$ distribution?

3) Other sources of ambiguity on the interpretation of tracer distribution in terms of circulation

$\mathrm{a} /$ Problems due to the lack of data or noise in the data

b/ The ocean may not be in steady state balance 
$\begin{array}{ll}\text { Summary and concluding remarks } & 175\end{array}$

Chapter VI - Summary and discussion. 


\section{Chapter I - Introduction.}

Great concern has been expressed about the possibility of drastic climate changes occurring in the middle of the next century (IPCC, 1990). Indeed, atmospheric concentrations of carbon dioxide $\left(\mathrm{CO}_{2}\right)$ and of other greenhouse gases have been rising at an exponential rate since the onset of the industrial revolution, and climate models predict that the increased greenhouse effect caused by these higher concentrations will result in a global warming of the lower atmosphere by at least $1.9^{\circ} \mathrm{C}$, and by potentially as much as $5.2^{\circ} \mathrm{C}$ (IPCC, 1990; warming predicted for a doubling of the partial pressure of $\mathrm{CO}_{2}$ in the atmosphere compared to preindustrial levels). By definition, these predictions cannot be verified. One can, however, test the ability of models to simulate climate changes by comparing observations of past climatic variations with model simulations of these variations.

Such a testing can be done using various paleo-climatic records, particularly records from the Last Ice Age, about 22000 years Before Present (BP). There is now ample evidence that climate was very different during this period (CLIMAP, 1976; 1981). Sea level was at 120 meters below the present level (Fairbanks, 1989). Northern Europe was covered with glaciers all the way to the British Isles (CLIMAP, 1976). Measurements of the oxygen isotopic composition in ice cores indicate that atmospheric temperatures were $7^{\circ} \mathrm{C}$ cooler over Central Greenland (Dansgaard et al., 1989). The partial pressure of carbon dioxide in the atmosphere $\left(\mathrm{pCO}_{2}\right)$ was about $2 / 3$ of its modern value (Jouzel et al., 1993).

The lower concentration of $\mathrm{CO}_{2}$ in the Last Ice Age atmosphere provides a case study of the effect of $\mathrm{CO}_{2}$ on the radiative balance and on climate. This effect has been simulated by Manabe and Bryan (1985) using a coupled ocean-atmosphere model. One interesting result of their study is the weakening of the thermohaline circulation in low $\mathrm{pCO}_{2}$ climates, which results in a reduced poleward transport of heat by the ocean. This reduced poleward heat transport is consistent with the comparatively cold climate observed in the Last Ice Age record of Northern Europe (Broecker et al., 1985). 
It has been argued that a change in the thermohaline circulation is also observed in the paleo-climatic record. Using Cadmium (Cd) and carbon $13\left({ }^{13} \mathrm{C}\right)$ data, Boyle and Keigwin (1982; 1987) and Duplessy et al. (1988) document a transfer of the nutrient maximum, located in the intermediate layers of the Atlantic in the modern ocean, to the deep layers of the Atlantic during the LGM. Because AABW is characterized by a relatively high nutrient content, they interpret the larger nutrient concentrations in deep waters as a consequence of a greater northward extent of Antarctic Bottom Water (AABW) in the deep glacial Atlantic, at the expense of waters formed in the North Atlantic. They link this larger volume of AABW to a reduced rate of formation of deep water in the North Atlantic during glacial times, which allows AABW to push further north. This reduction in the rate of formation of NADW is qualitatively consistent with the weakening of the thermohaline circulation obtained by Manabe and Bryan (1985).

It is difficult, however, to interpret the paleo-data in a quantitative manner. First, it is not clear how large are the differences between the volumes of the water masses in the modern and in the glacial ocean. The data coverage of the LGM Atlantic is still incomplete, with few data points in the Western Atlantic and in the South Atlantic. Moreover, interpreting changes in the extent of the various water masses in terms of circulation changes is not a straightforward undertaking. A clear distinction must be made between the water mass distribution implied by the paleo-tracers, and the rates of formation of these water masses. It is not obvious, for instance, whether large circulation changes are required to explain observed changes in tracer distributions. A comparatively slight reduction of the rate of formation of Lower NADW during the LGM may be sufficient to allow AABW to fill most of the deep Atlantic, especially if the reduction is sustained for thousands of years.

The objective of the work presented here is to determine whether the available paleoceanographic data are sufficient to establish in a quantitative manner the extent to which the circulation in the deep Atlantic was different during the Last Ice Age. Note that the issue of understanding how the ocean can switch from the glacial to the modern tracer distribution is not addressed. Modeling the glacial-interglacial transition would require the compilation of detailedtime series of the tracer distributions over several thousand years. Such a compilation is not available yet (existing compilations usually produce a LGM and a Holocene value only). Thus, 
this work is only concerned with the comparison of the modern and the glacial circulations, which are assumed to be in steady state, but not with the transition from one circulation to another.

In chapter $\Pi$, an attempt is made to quantify the extent of the change in volume of AABW and NADW implied by the ${ }^{13} \mathrm{C}$ data. The nature of the paleo-data, and their geographical coverage, is also discussed in this chapter. The rest of the thesis concerns the interpretation of the water mass distribution, as indicated by the paleo-tracers $\delta^{13} \mathrm{C}$ and $\delta^{18} \mathrm{O}$, in terms of circulation changes ( $\delta^{13} \mathrm{C}$ and $\delta^{18} \mathrm{O}$ are related to the isotopic composition of foraminifera shells from which the paleo-data are obtained, as explained in chapter II). The interpretation of the paleo-tracers is done using an inverse model of the circulation of the Atlantic, described in chapter IV. Using an optimization procedure, introduced in the previous chapter, the model is constrained to best-fit the paleo-data. Three model runs are described in chapter V. The first run estimates the modern ocean circulation, which is then used as a reference to which paleo-circulations can be compared. The second run consists in trying to find the smallest changes in the modern ocean circulation required to make it compatible with the water mass distribution implied by the paleo-data. Finally, the third run examines if a reduction of $50 \%$ of the flux of Lower NADW is consistent with the paleo-data. The results of these runs and their implications for the interpretation of paleo-tracer data in terms of oceanic circulation are discussed in part C of chapter V. 


\section{Chapter II - Geochemical evidence for a reduced volume of Lower North Atlantic Deep Water during the Last Glacial Maximum.}

In this chapter, the evidence for different water mass distributions in the modern and in the glacial Atlantic is examined. The paleo-tracer carbon 13, which can be thought of as a proxy for nutrients, is used to identify the water masses North Atlantic Deep Water (NADW) and Antarctic Bottom Water $(\mathrm{AABW})$ in the glacial ocean. These water masses are viewed as volumes of water delimited by transition zones. These transition zones are defined as rigorously as possible in order to compare the volumes of NADW and $\mathrm{AABW}$ in the glacial and in the modern Atlantic.

The chapter outline is as follows: part A is an overview of the data available for the LGM. Part B describes the nutrient and carbon cycles which involve paleo-geochemical tracers such as carbon 13. Part $\mathrm{C}$ presents a compilation of carbon 13 and oxygen 18 data for the Last Ice Age. Finally, part D examines different reconstructions of the water mass distribution in the Glacial Atlantic, and compares them to the present distribution.

\section{A - The data}

Much of the data available in paleoceanography are geochemical tracer distributions inferred from measurements in the shells of microscopic animals called foraminifera. These animals live either at the surface of the ocean (planktonic foraminifera), or at the bottom of the ocean (benthic foraminifera). They grow shells made of calcium carbonate $\left(\mathrm{CaCO}_{3}\right)$ whose isotopic composition reflects that of the surrounding waters. When foraminifera die, their shells sink and are incorporated in the sediments. It is possible to retrieve these shells in sediment cores, and by analyzing them to reconstruct the distribution of various geochemical tracers in ancient oceans.

It is also possible to estimate past Sea Surface Temperature (SST) patterns from the study of the geographical distribution of the shells of different species of planktonic foraminifera. Planktonic foraminifera are sensitive to temperature, and different species grow in different 
temperature environments. SST in the LGM world ocean has been reconstructed (CLIMAP, 1976; 1981) assuming that the relation linking the distribution of planktonic foraminifera to SST in the glacial ocean was similar to that in the modern ocean. This method is not applicable to the deep ocean since temperature gradients appear to be too small there to influence the geographical distribution of benthic foraminifera.

Foraminifera only live in the upper layers and at the bottom of the ocean, and there is no information available about the rest of the water column. The problem of studying intermediate waters can be partly solved by looking at the sediments around islands, seamounts, ridges and continental margins.

The first two sections of part A describe in greater detail the different types of data available in the deep and upper oceans. Section 3 examines the uncertainties associated with these data. Section 4 concludes with a short overview of other data sources, namely glaciers and land records.

\section{1) Deep water geochemical tracers}

Empirical studies have determined the relation between the composition of benthic foraminifera shells and the composition of sea water. Foraminifera species are chosen so that they really represent the composition of the ocean. For instance, species which live inside sediments should not be considered since the chemical composition of the sediments is different from that of the overlying water. The Cibicidoides species, which has been shown to live above the sediment/water interface (Lutze and Thiel, 1987), is thought to reliably record chemical tracers present in sea water, and is the most widely used foraminifera.

An extensive review of deep ocean paleoceanography is given in Boyle (1990). The most commonly used tracers are summarized here.

a/ $\delta^{18} \mathrm{O}$, a proxy for global ice volume and temperature:

$\delta^{18} \mathrm{O}$ is defined as: 


$$
\delta^{18} \mathrm{O}=\left\{\frac{\left\langle\frac{\left[{ }^{18 \mathrm{O}]}\right.}{\left[{ }^{16 \mathrm{O}]}\right]}\right\rangle_{\text {sample }}}{\left[\frac{{ }^{18 \mathrm{O}]}}{\left[{ }^{16 \mathrm{O}]}\right]}\right\rangle_{\text {standard }}}-1\right\} \times 1000
$$

where:

$$
\left\langle\frac{[18 \mathrm{O}]}{[16 \mathrm{O}]}\right\rangle_{\text {standard }}
$$

is the ratio of ${ }^{18} \mathrm{O}$ over ${ }^{16} \mathrm{O}$ in a reference sample (usually PDB from the Pee Dee Belemnite formation in the United States, or SMOW, Standard Mean Ocean Water); [18 O] and [16 O] indicate the quantity of the oxygen isotopes 18 and 16 present in the sample.

The ratio of ${ }^{18} \mathrm{O}$ over ${ }^{16} \mathrm{O}$ is measured by mass spectrometry and indicates the relative proportion of the oxygen 18 isotope present in foraminifera shells $\left(\mathrm{CaCO}_{3}\right)$, in water molecules $\left(\mathrm{H}_{2} \mathrm{O}\right)$, or in the total dissolved inorganic carbon of sea water $\left(\mathrm{CO}_{2}\right)$. This ratio being close to one, it is convenient to use the $\delta^{18} \mathrm{O}$ notation (if the ratio is 1.001 , then $\delta^{18} \mathrm{O}=1 \%$ ).

There is some information about the $\delta^{18} \mathrm{O}$ of sea water during the LGM because the $\delta^{18} \mathrm{O}$ of calcium carbonate, which constitutes foraminifera shells, is nearly in equilibrium with that of sea water. There is an offset, however, between the values of $\delta^{18} \mathrm{O}$ in foraminifera shells and the values of $\delta^{18} \mathrm{O}$ in water molecules which depends on the water temperature. Additionally, different species show variable departures from equilibrium as well.

The $\delta^{18} \mathrm{O}$ values recorded in foraminifera which grew during the Last Ice Age are generally higher than the $\delta^{18} \mathrm{O}$ values recorded in foraminifera which grew recently, because the volume of ice stored in continental glaciers was larger and oceanic temperatures were lower during glacial times. About two thirds of the difference between modern and glacial values is due to ice volume changes. The water which composes high latitude atmospheric precipitation is depleted in oxygen 18 because of a distillation process occurring in the evaporation-precipitation cycle (Mix, 1987). Thus, glaciers, which are produced by atmospheric precipitation, are depleted in oxygen 18 , and hence for larger continental glaciers, more oxygen 18 is left in the ocean. Using sea level reconstructions (Fairbanks, 1989), the volume of the ocean during the LGM, and therefore that of the continental ice sheets, can be calculated quite accurately. Assuming a particular average $\delta^{18} \mathrm{O}$ 
for the ice, the volume difference between glacial and modern times translates to a difference between the mean $\delta^{18} \mathrm{O}$ of sea water which is recorded in foraminifera shells.

Most of the remaining one third of the temporal change of $\delta^{18} \mathrm{O}$ is due to lower temperatures in the deep ocean in glacial times. Indeed, the $\delta^{18} \mathrm{O}$ recorded in foraminifera shells increases when water temperature decreases, by about $0.25 \%$ oper ${ }^{\circ} \mathrm{C}$, and it has been shown that the deep ocean was, on average, $2^{\circ} \mathrm{C}$ cooler during the last glacial maximum (see review by Mix, 1987).

\section{b/ Nutrient related tracers}

Various geochemical tracers are related to the nutrient content of sea water. For paleooceanographic purposes, the main ones are carbon 13 and Cadmium (Cd).

The $\delta^{13} \mathrm{C}$ of the $\mathrm{CaCO}_{3}$ which constitutes foraminifera shells and the $\delta^{13} \mathrm{C}$ of total inorganic carbon in sea water can be measured by mass spectrometry. $\delta^{13} \mathrm{C}$ is defined in the same way as $\delta^{18} \mathrm{O}:$

$$
\delta^{13} C=\left\{\frac{\left\langle\frac{\left[{ }^{13 C}\right]}{\left[{ }^{12 C}\right]}\right\rangle_{\text {sample }}}{\left[\frac{\left.{ }^{13 C}\right]}{\left[{ }^{12 C}\right]}\right\rangle_{\text {standard }}}-1\right\} \times 1000
$$

where:

$$
\left\langle\frac{\left[{ }^{13} \mathrm{C}\right]}{\left[{ }^{12} \mathrm{C}\right]}\right\rangle_{\text {standard }}
$$

is the ratio of carbon 13 over carbon 12 in a standard sample (usually PDB). $\left[{ }^{12} \mathrm{C}\right]$ and $\left[{ }^{13} \mathrm{C}\right]$ are the concentrations of total inorganic carbon 12 and carbon 13 in samples of sea water or in samples of calcium carbonate. The concentration of total inorganic carbon in samples of sea water, $\Sigma \mathrm{CO}_{2}$, is the sum of the concentrations of dissolved $\mathrm{CO}_{2}$, carbonate $\left(\mathrm{CO}_{3}{ }^{2-}\right)$ and bicarbonate $\left(\mathrm{HCO}_{3}{ }^{-}\right)$ions. Thus the $\delta^{13} \mathrm{C}$ of sea water is the $\delta^{13} \mathrm{C}$ of the sum of these three species. Figure II-A-1 shows that $\delta^{13} \mathrm{C}$ inversely correlates with phosphate (PO4). This inverse correlation is a 


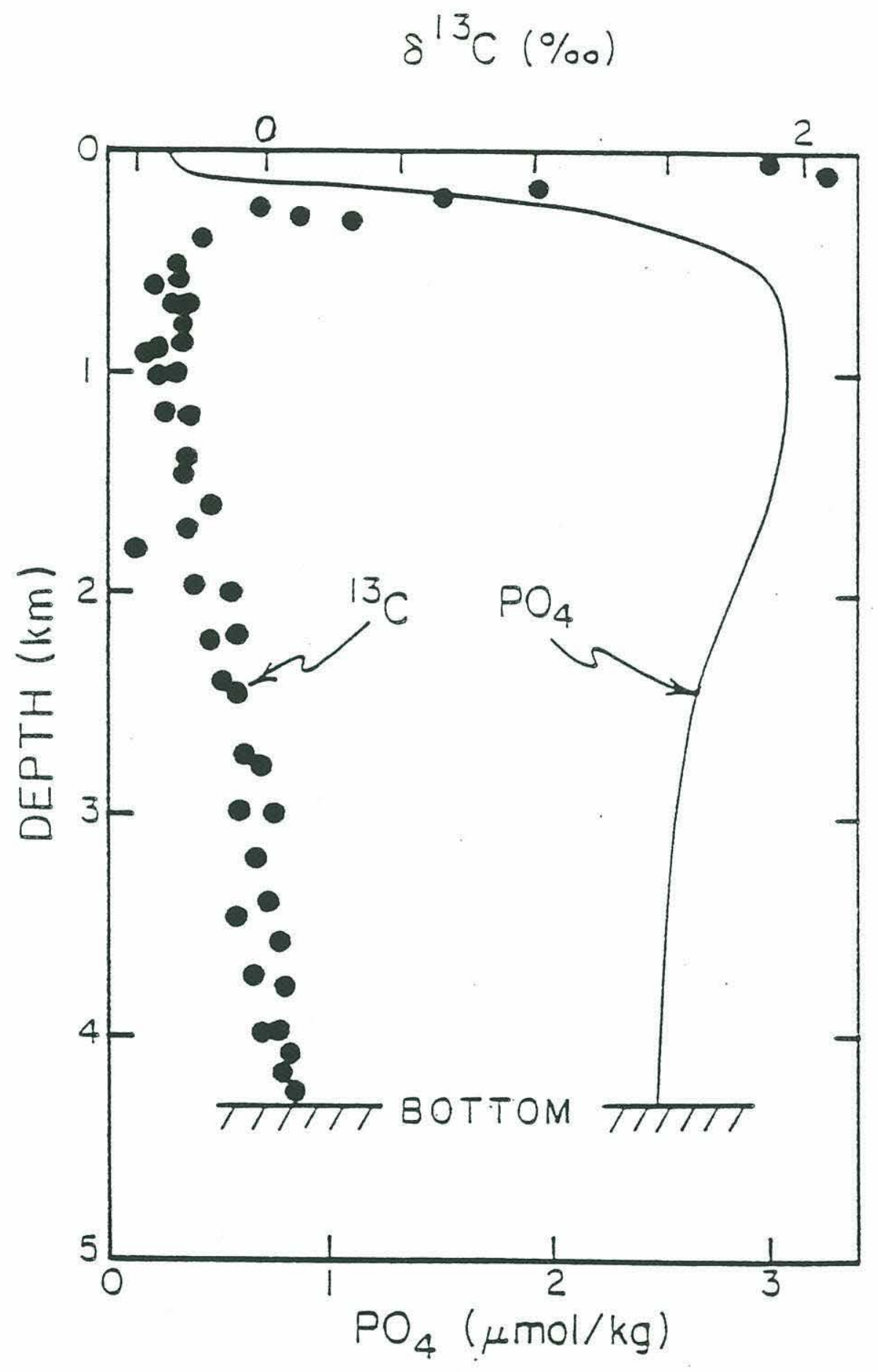

Figure II-A-1: Plots of $\delta^{13} \mathrm{C}$ and $\mathrm{PO}_{4}$ versus depth at GEOSECS station $346\left(28^{\circ} \mathrm{N}\right.$, $121^{\circ} \mathrm{W}$ ) in the northwestern Pacific Ocean. The ${ }^{13} \mathrm{C}$ analyses were made by Craig and Kroopnick at the Scripps Institution of Oceanography, and the $\mathrm{PO}_{4}$ analyses as part of the GEOSECS program. Figure from Broecker and Peng (1982). 
consequence of biological activity in the upper ocean: planktonic organisms take up carbon 12 preferentially to carbon 13 (this phenomenon is called biological fractionation). Thus, when these organisms die, they sink and carry $\delta^{13} \mathrm{C}$ depleted organic matter to the deep ocean. At the same time, this biological pump (downward flux of organic matter through the sinking of dead organisms, and fecal pellets) transports nutrients to the deep water. So, the stronger the biological pump is, the more depleted in carbon 13 and the more enriched in nutrients the deep waters are, whence the inverse relationship between $\delta^{13} \mathrm{C}$ and $\mathrm{PO} 4$.

Benthic foraminifera substitute $\mathrm{Cd}$ for $\mathrm{Ca}$ in the crystal lattices of their calcium carbonate shells in proportion to the bottom water cadmium concentration. The cadmium concentration is approximately linearly related to the phosphate concentration in the deep ocean (Boyle et al., 1976; Bruland et al., 1978) and, like $\delta^{13} \mathrm{C}$, it reflects the distribution of nutrients. Note that there are some discrepancies between $\delta^{13} \mathrm{C}$ and $\mathrm{Cd}$ data in several regions of the ocean; in the Southern Ocean, for instance, $\mathrm{Cd}$ data indicate that the nutrient content of AABW is about the same during glacial and interglacial times, whereas $\delta^{13} \mathrm{C}$ data indicate that the nutrient content of $\mathrm{AABW}$ is higher during glacial periods (Boyle, 1992). These discrepancies are not yet explained (see part IID).

\section{c) $\Delta^{14} \mathrm{C}$, a radioactive tracer}

The carbon 14 content of sea water may be expressed using the $\delta$ notation, as for $\delta^{13} \mathrm{C}$ :

$$
\delta^{14} C=\left\{\frac{\left\langle\frac{\left[\mathrm{C}^{14}\right]}{\left[\mathrm{C}^{12}\right]}\right. \text { sample }}{\left\langle\frac{\left.\mathrm{C}^{14}\right]}{\left[\mathrm{C}^{12}\right]}\right. \text { standard }}-1\right\} \times 1000
$$

In practice, however, $\Delta^{14} \mathrm{C}$ is used. $\Delta^{14} \mathrm{C}$ is the per mil difference from the ${ }^{13} \mathrm{C} /{ }^{12} \mathrm{C}$ ratio in the atmosphere prior to the onset of the industrial revolution (i.e. about 1850) and normalized to a constant ${ }^{13} \mathrm{C} /{ }^{12} \mathrm{C}$ ratio:

$$
\Delta^{14} \mathrm{C}=\delta^{14} \mathrm{C}-2\left(\delta^{13} \mathrm{C}+25\right)\left(1+\delta^{14} \mathrm{C} / 1000\right)
$$

Carbon 14 is created in the atmosphere by the action of cosmic rays on atmospheric nitrogen 14. The carbon 14 content of surface waters in regions of deep water formation is set by the 
balance between the transfer of atmospheric ${ }^{14} \mathrm{C}$ and mixing with older deep waters. Once it has sunk, deep water is isolated from the atmosphere and its carbon 14 content starts decreasing. Thus it is possible to estimate the "age" of a water mass in the modern ocean from radiocarbon dating. In paleoceanography, the age of a water mass is approximately given by the difference between the $\Delta^{14} \mathrm{C}$ of planktonic and benthic foraminifera which grew at the same time. This approach requires precise measurements on small samples ${ }^{1}$, which were impossible before Accelerator Mass Spectrometers became available in the early 1980 's. This capability of dating water masses makes carbon 14 a powerful constraint for the estimation of the rate of ventilation of the deep ocean during the LGM. However, there are still very few data points and the data base is only growing slowly because of the cost of making the measurements (it is about 20 times more expensive to measure ${ }^{14} \mathrm{C}$ than ${ }^{13} \mathrm{C}$ ). The potential of this technique is least in the Atlantic where deep waters are rapidly flushed and do not age much (aging of the order of a hundred years in the modern ocean, Broecker, 1981).

\section{d/ The conventionally accepted interpretation of deep water paleo-tracers}

Both $\delta^{13} \mathrm{C}$ and $\mathrm{Cd}$ data show that, compared to the modern ocean, the deep North Atlantic was enriched in nutrients during the LGM (Boyle, 1992). This enrichment has been interpreted as being due to a reduced production of Lower NADW which resulted in a reduced volume of Lower NADW and allowed nutrient-rich $\mathrm{AABW}$ to penetrate further north during the LGM (Boyle and Keigwin, 1982; 1987; Duplessy et al., 1988). A reduction in the volume of Lower NADW is consistent with the fact that the ${ }^{14} \mathrm{C}$ age difference between planktonic and benthic foraminifera in the Equatorial Atlantic went from 675 years ( \pm 80 years) during the LGM (Broecker et al., 1990) down to 350 years today. Indeed, $A A B W$ is older than NADW, and having AABW pushing further north should result in an increase in the planktonic-benthic foraminifera age difference. $\delta^{13} \mathrm{C}$ and $\mathrm{Cd}$ data also indicate a lower nutrient content in intermediate waters in the Northern and Equatorial Atlantic. This lower nutrient content has been interpreted as being due to an increased

\footnotetext{
1 The water mass age is the small difference between two large numbers: for the LGM, foraminifera shells are about twenty thousand years old but the age difference between planktonic and benthic foraminifera is no larger than a few thousand years.
} 
production of nutrient-depleted Upper NADW during the LGM (Boyle and Keigwin, 1987; Duplessy et al., 1988).

The conventional interpretation of the paleo-tracers in terms of production of deep water in the glacial Atlantic is challenged in chapter V.

\section{2) Surface water}

As mentioned above, a SST map has been reconstructed in the LGM world ocean using the geographical distribution of planktonic foraminifera as a proxy for temperature (CLIMAP, 1976; 1981; Figure II-A-2). This map shows that the polar fronts were shifted equatorward during the ice age. Apparently, the Gulf Stream was more zonal (Keffer et al., 1988), which suggests that the northward flow of surface water necessary to compensate deep water formation in the Norwegian Sea decreased. Such a decrease is intuitively consistent with a reduced rate of formation of Lower North Atlantic Deep Water. Deep water formation, however, could take place somewhere else, south of Iceland for instance. Thus the lack of a surface flow does not prove that there was a reduced production of Lower NADW in the North Atlantic.

There are also measurements of $\delta^{18} \mathrm{O}$ and $\delta^{13} \mathrm{C}$ in planktonic foraminifera shells. However, the interpretation of geochemical tracers in the upper ocean in terms of water mass distribution is difficult because of the large temporal and spatial variability in the upper few hundred meters of the ocean. Moreover, Keigwin and Boyle (1989) have analyzed coretop samples from the Atlantic, the Pacific and the Southern Ocean, and argue that there is no significant correlation between the $\delta^{13} \mathrm{C}$ of the $N$. Pachyderma foraminifera species and that of sea water in the data they analyzed. The fact that this species has been widely used to study the $\delta^{13} \mathrm{C}$ of surface waters cast some doubts on the validity of the surface water geochemical data set. Other workers have different views (Labeyrie et al., 1985), and the capability of planktonic foraminifera to record $\delta^{13} \mathrm{C}$ is still a matter of controversy. 


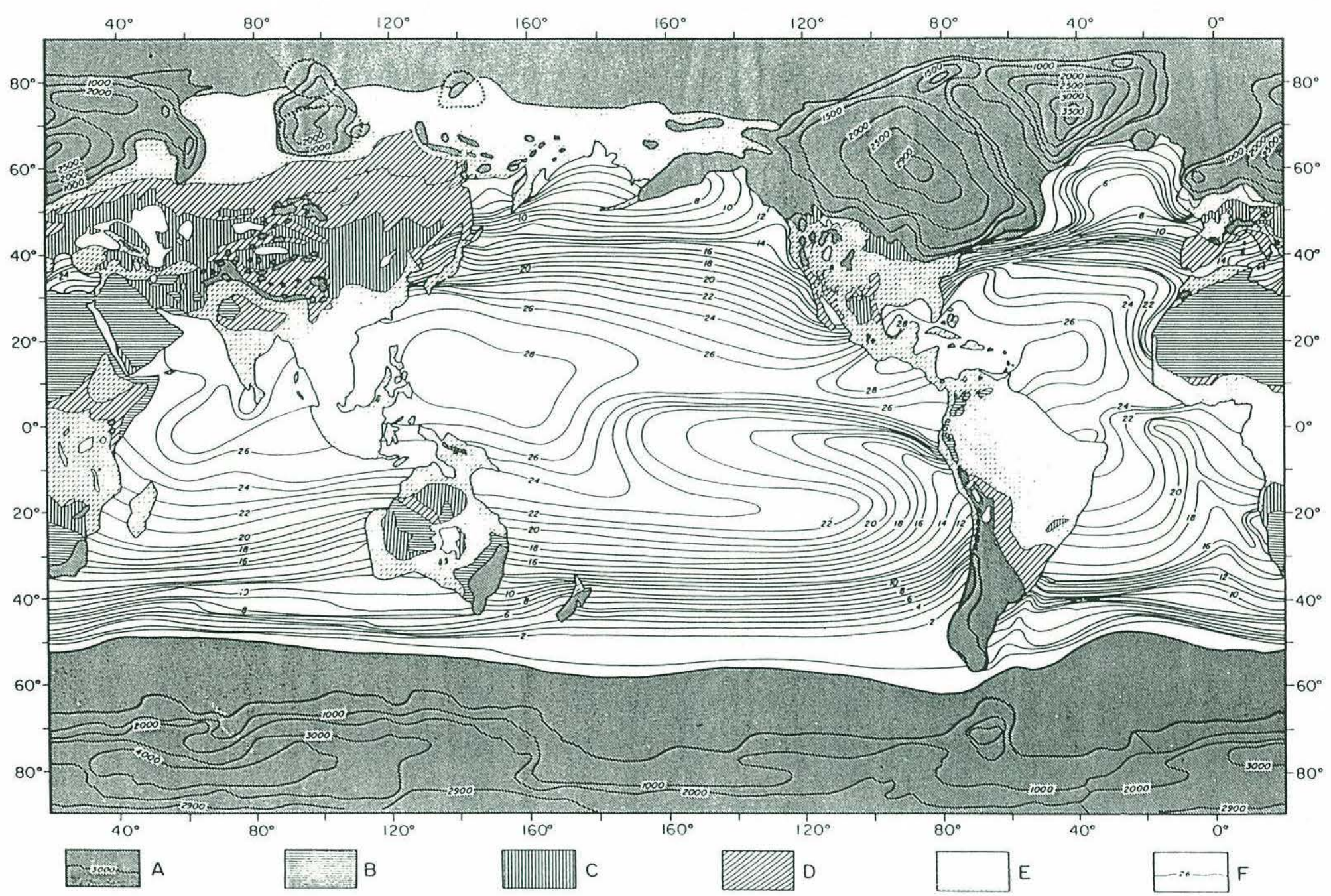

Figure II-A-2: Reconstruction of sea surface temperature during the LGM (in August).

Figure from CLIMAP (1976). 


\section{3) A vailability and quality of oceanic climate indicators}

Paleoceanography is a young science, and each measurement requires a large amount of work, both to collect sediment cores and to analyze them in the laboratory; thus, the set of measurements available for the LGM is relatively small compared to that of the modern ocean. The LGM North Atlantic is the best documented paleo-ocean: there are more than a hundred estimates of SST and of deep sea $\delta^{13} \mathrm{C}$ and $\delta^{18} \mathrm{O}$, a few tens of Cadmium data points and only four published measurements of carbon 14.

It is not possible to obtain data from the deep glacial ocean, below the depth called the Calcium Compensation Depth (CCD). The CCD is defined as the depth below which almost no calcium carbonate is preserved. Just as snow is preserved on top of terrestrial mountains, high marine topographic features are blanketed by a layer of calcium carbonate rich sediments. As depth increases, there is less and less calcium carbonate preserved in the sediments because the solubility of $\mathrm{CaCO}_{3}$ in sea water increases with pressure and with colder temperatures. Dissolution does not begin abruptly at the CCD. There is a zone of transition located between the lysocline (depth at which dissolution begins, several hundred meters above the CCD) and the CCD. In this zone of transition, only part of the calcium carbonate reaching the sediments is dissolved. Since foraminifera shells are made of $\mathrm{CaCO}_{3}$, there are essentially no data preserved below the CCD. In the modern Atlantic, foraminifera shells are preserved almost everywhere because the CCD is at a depth of about $5 \mathrm{~km}$. In the North Pacific, however, the CCD is shallower, and foraminifera shells are preserved at shallower depths only. Note that the CCD and the lysocline are though to have been deeper in the Pacific and shallower in the Atlantic during the LGM (Broecker and Peng, 1982). Thus, there might be some data preserved below the modern Pacific CCD and no LGM data preserved just above the modern Atlantic CCD.

There are fewer measurements of paleo-tracers available in the Pacific and Southern oceans. One difficulty in the Southern Ocean is the scarcity of foraminifera because the presence of large quantities of silica enables diatoms and radiolaria to flourish at the expense of foraminifera. 
Most paleo-records can go back to 200,000 years Before Present (BP), some to $2 \times 10^{6} \mathrm{BP}$, and some even further (several tens of million years). Carbon 14 records, however, are limited to about 25,000 years BP (the half life of ${ }^{14} \mathrm{C}$ is 5730 years, and after 25,000 years, there is not enough ${ }^{14} \mathrm{C}$ left to make reliable measurements using accelerator mass spectrometers).

The analytical precision of $\delta^{13} \mathrm{C}$ measurements is about $0.05 \%$, and that of $\delta^{18} \mathrm{O}$ is better than $0.1 \%$. However, there are other sources of uncertainty such as bioturbation which are larger than the analytical precision. Bioturbation is the mixing of the upper 10 centimeters, or so, of the sediments by fauna living there. The average sedimentation rate of the world ocean being 2 to 5 cm per thousand years (Broecker and Peng, 1982), bioturbation limits the time resolution of most sediment cores to a few thousand years. Because it acts as a low-pass filter, bioturbation smoothes out the extrema of $\delta^{18} \mathrm{O}$ and $\delta^{13} \mathrm{C}$ values characterizing the Last Ice Age, and therefore introduces some error in the LGM data. Quantifying this error is difficult because it requires information about sedimentation rates and models of the effects of mixing on the tracer records. Thus, most observations are published without observational uncertainties, and the reader is left to exercise his own judgment. There are a few high sedimentation rate cores (Lehman and Keigwin, 1992, for instance) which can accumulate deposits up to a rate of $200 \mathrm{~cm}$ per thousand years (due to the drift of fine grained material towards a particular site), and can record changes occurring over a time scale of a hundred years. These records are little affected by bioturbation. Note that bioturbation creates severe artefacts in ${ }^{14} \mathrm{C}$ measurements, which rules out the use of ${ }^{14} \mathrm{C}$ in all but higher sedimentation rate sediment cores.

Another source of error in $\delta^{13} \mathrm{C}$ records is the fallout of organic matter on the sediments in high productivity regions. Organic matter is depleted in $\delta^{13} \mathrm{C}$, and since a significant fraction of this organic matter oxydizes when reaching the sediment-water interface, it tends to lower the $\delta^{13} \mathrm{C}$ of the bottom water in which foraminifera grow. In this case, the $\delta^{13} \mathrm{C}$ recorded in foraminifera shells is representative of the water a few millimeters above the sediment-water interface, but not of the overlying water mass (Mackensen et al., 1993). It is also difficult to quantify this source of error. In low productivity regions, essentially all the organic matter dissolves in the upper and intermediate layers of the ocean, and does not influence the $\delta^{13} \mathrm{C}$ 
record. Thus the error introduced by fallout of organic matter is large only in high productivity regions.

The observational uncertainties must be estimated in order to use the data in the inverse model described in chapter IV. In part C of this chapter, an uncertainty of $0.2 \%$ is estimated for both $\delta^{13} \mathrm{C}$ and $\delta^{18} \mathrm{O}$ data. Because making an individual estimate for every published data point is beyond the scope of this study, the uncertainty due to bioturbation, fallout of organic matter, and the limited analytical precision of the measurements is assumed to be the same for every data point. This assumption is not very realistic because different cores have different accumulation rates (thus are affected by bioturbation in different ways), and biological productivity is greatly different from one region to another. Generally speaking, cores located below mid-latitude gyres are more likely to be affected by bioturbation because of the low sedimentation rates there; cores located near polar fronts in the Southern Ocean and cores from the upwelling regions off the African coast are more likely to be affected by fallout of organic matter.

\section{4) Land and atmospheric records}

It is possible to estimate atmospheric climatic fluctuations from several types of observations. To name only a few, bubbles of air trapped in high latitude glaciers produce information about the chemical composition of ancient air (Jouzel et al., 1993). The isotopic composition of ice can be interpreted in terms of atmospheric temperature (Dansgaard et al., 1989). Geological evidence provides information about the extent of glaciers, the position of the snow line, and sea level (CLIMAP, 1981). The ${ }^{14} \mathrm{C}$ content of Uranium-Thorium dated corals can be used to calibrate the ${ }^{14} \mathrm{C}$ time scale, which in turn provides a chronology of various climatic events since the LGM (Bard et al., 1990).

\section{B - Geochemical cycles in the ocean}

We have seen in part A that most of the LGM deep ocean data consist of measurements of $\delta^{13} \mathrm{C}$ and $\delta^{18} \mathrm{O}$ in foraminifera shells. We would like to interpret these measurements in terms of 
circulation changes so it is worthwhile to examine how the geochemical cycles of these tracers are influenced by the ocean circulation.

The nutrient cycle is described in section 1, and the concept of preformed nutrients is introduced in section 2 . Section 3 shows that the same concept applies to $\delta^{13} \mathrm{C}$, and explains how preformed values of this tracer can be used to identify the different water masses.

\section{1) The nutrient cycle}

Nutrients are used in the upper layers of the ocean by biological organisms to grow their organic tissues. Organic matter is in turn transferred to the deeper layers of the ocean under the form of sinking particles composed of dead organisms and fecal pellets. Most of these particles are dissolved during the early stages of their descent and do not leave the upper ocean. However, about $10 \%$ of the organic particle flux reaches the deep ocean. Of these $10 \%$, nine tenths are remineralized (dissolved in sea water), and only one tenth is incorporated into the sediments (Broecker and Peng, 1982). This remineralization can be described by the following chemical reaction:

$$
\mathrm{C}_{\beta} \mathrm{H}_{\xi} \mathrm{O}_{\varphi} \mathrm{N}_{\alpha} \mathrm{P}+\gamma \mathrm{O}_{2} \Leftrightarrow \mathrm{H}_{3} \mathrm{PO}_{4}+\alpha \mathrm{HNO}_{3}+\beta \mathrm{CO}_{2}+\lambda \mathrm{H}_{2} \mathrm{O}
$$

The stoichiometric coefficients $\alpha, \beta$, and $\gamma$ are the so-called "Redfield Ratios" and are relatively uniform throughout the world ocean, with $\alpha \approx 16, \beta \approx 117$, and $\gamma \approx 170$ (Anderson and Sarmiento, 1994). Thus, deep waters are enriched in phosphate, carbon, and other nutrients but depleted in oxygen as they flow away from their regions of formation. Eventually, they upwell and return the nutrients to the surface of the ocean, and the cycle can be repeated. One can see in Figure II-B-1 that waters formed in the deep North Atlantic are the most depleted in nutrients such as nitrate, and that they are gradually enriched along their way to the Pacific. The nutrient content 


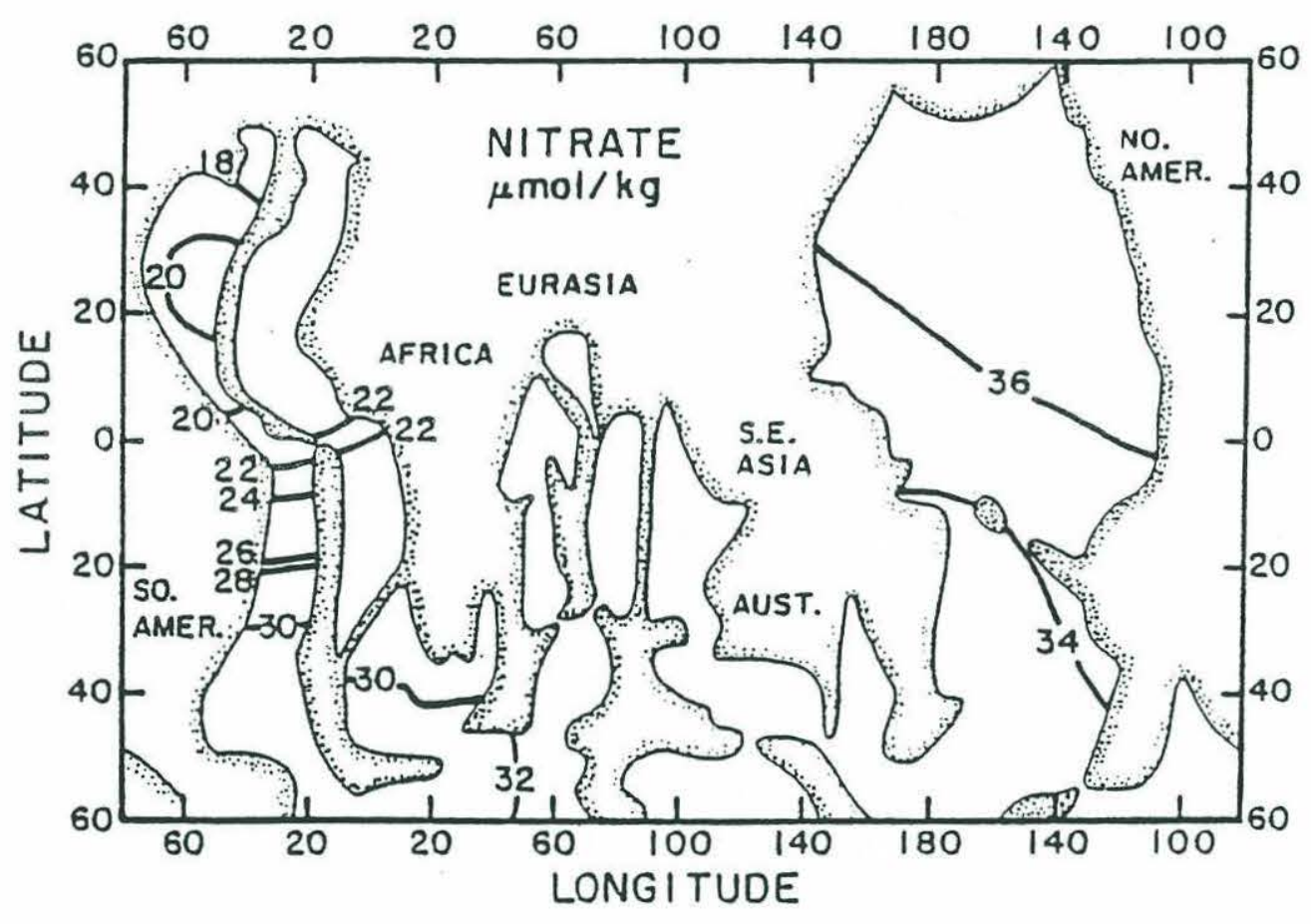

Figure II-B-1: Sketch of the distribution of dissolved nitrate at a depth of 4000 meters in the world's major ocean basins. Based on results obtained during the GEOSECS program. From Broecker and Peng (1982). 
of deep water formed in the North Atlantic, however, is different from the nutrient content of deep water formed in the Southern Ocean, because different sources of deep water have different preset nutrient contents.

Because the biological particle flux also contains the shells of planktonic foraminifera and of some other biological organisms, it transports calcium carbonate (inorganic matter) to the deep ocean. Almost all of this sinking inorganic material reaches the deep ocean. A large fraction of it reaches the sediment-water interface in regions located below the lysocline, and dissolves. Only $20 \%$ of the initial production of calcium carbonate is preserved in the sediments. Note that the remineralization of organic matter and calcium carbonate takes place at different depths.

\section{2) Preformed nutrients}

Mid-latitude surface waters are almost completely depleted in nutrients by biological activity, but not high latitude surface waters where deep water is formed. The nutrients are not fully utilized in high latitudes because of the lack of light in the winter and because the mixed layer is so deep that biological organisms are constantly entrained to depths where not enough light is available for photosynthesis to occur (Broecker and Peng, 1982). It has also been hypothesized that there is not enough iron in the Southern Ocean so biological activity reaches its limits before all the nutrients are consumed (Martin et al., 1990). Therefore, high latitude surface waters have a non-zero nutrient content. When deep water is formed, this nutrient surplus, called "preformed nutrients", is transferred to the deep ocean and can be used to trace the different water masses.

In the modern ocean, water masses formed in the Southern Ocean have larger preformed nutrients than waters formed in the North Atlantic, because surface waters from which the water masses originate are less depleted in nutrients in the Southern Ocean than in the North Atlantic. In fact, the nutrient content of NADW increases on its way to the Southern Ocean less because of aging due to the particle flux, than because NADW mixes with the nutrient-rich $A A B W$ and AAIW. 
Therefore, in order to interpret the nutrient distribution in terms of relative importance of the different sources of deep water, one needs to know the preformed nutrient concentration of each source.

\section{3) Preformed $\delta^{13} \mathrm{C}$}

As with nutrients, $\delta^{13} \mathrm{C}$ is closely linked to biological activity, so we expect this tracer to have different preformed values characterizing different water masses. However, unlike nutrients, its cycle is further complicated by exchanges with the atmosphere and by the carbonate chemistry.

\section{a/ Biological fractionation}

The fact that biological organisms preferentially take up light isotopes to make their organic tissues (typically, organic matter has a $\delta^{13} \mathrm{C} 20 \%$ lower than that of the surrounding waters) results in high $\delta^{13} \mathrm{C}$ values in surface waters and low $\delta^{13} \mathrm{C}$ values in deep waters. Because nutrients are removed more efficiently in the Northern Atlantic than in the Southern Ocean, preformed values of $\delta^{13} \mathrm{C}$ are higher in NADW than in $\mathrm{AABW}$, by about $0.7 \%$, as shown in Figure II-B-2 (Kroopnick, 1985). As in the case of phosphate, there is an aging trend as a parcel

of water goes from the North Atlantic to the North Eastern Pacific (Figure II-B-3): $\delta^{13} \mathrm{C}$ decreases from the North Atlantic to the North Pacific because of the fallout of organic matter. Particle remineralization in the deep ocean releases ${ }^{13} \mathrm{C}$ depleted organic matter, thereby decreasing the $\delta^{13} \mathrm{C}$ of deep waters, at the same time as it consumes oxygen. Thus, the values of $\delta^{13} \mathrm{C}$ and of $\mathrm{O}_{2}$ are strongly correlated in the ocean, and $\delta^{13} \mathrm{C}$ can be thought of as a proxy for oxygen.

\section{b/ Ocean-Atmosphere exchanges}

Unlike phosphorus or other nutrients, there is a significant exchange of carbon 13 with the atmosphere which complicates the cycle. The exchange of carbon between the atmosphere and the 

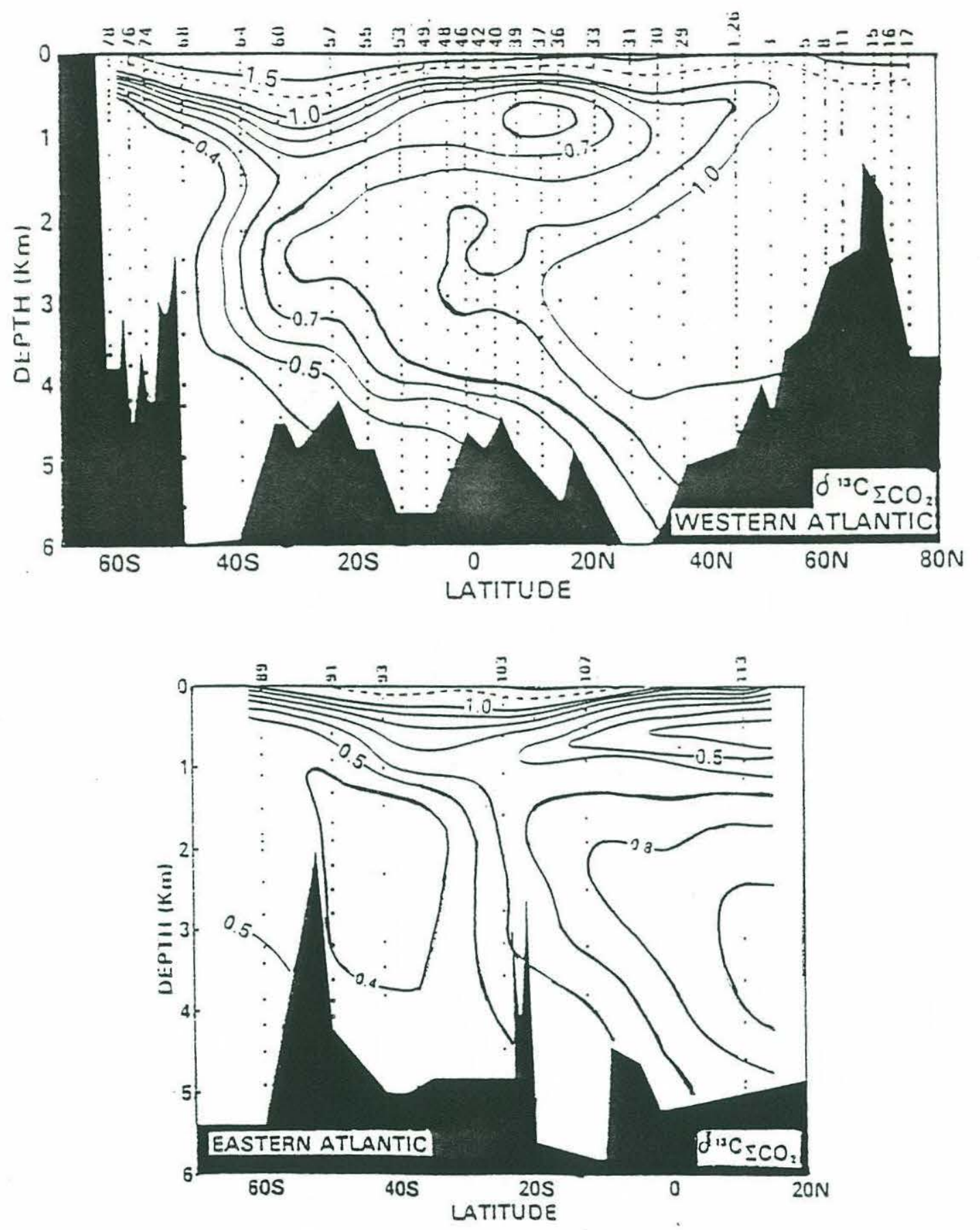

Figure II-B-2, top panel: Latitudinal distribution of $\delta^{13} \mathrm{C}$ versus depth for the Western Atlantic Ocean. bottom panel: Latitudinal distribution of $\delta^{13} \mathrm{C}$, as in top panel, except for the Eastern Atlantic Ocean. Station numbers are indicated across the top. The dots indicate depths at which samples were collected. The contour interval is $0.1 \%$. See GEOSECS Atlas for location of stations (GEOSECS,1980). Figures from Kroopnick (1985). 


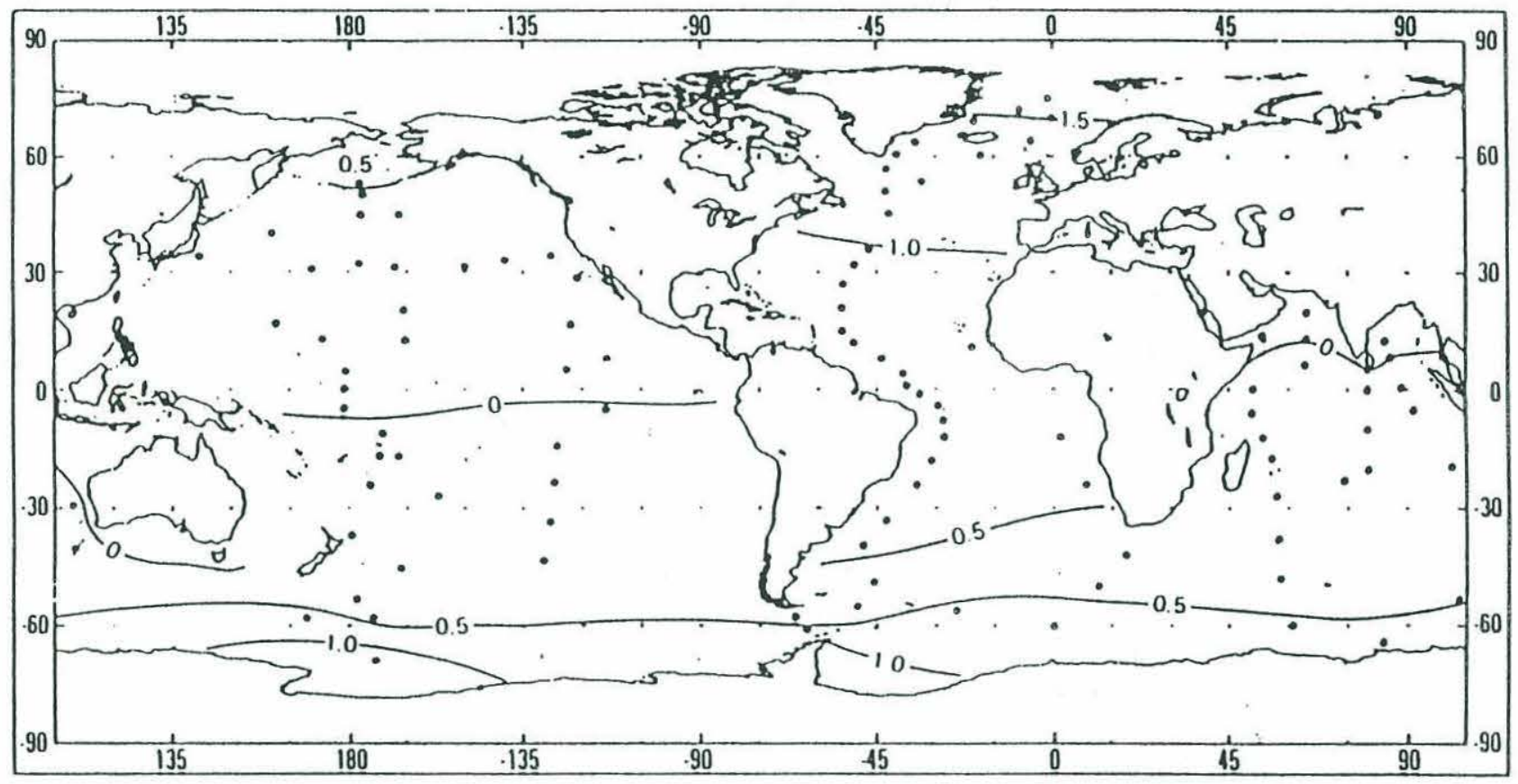

Figure II-B-3: Horizontal projection of $\delta^{13} \mathrm{C}$ at a depth of between $200(0)$ and $2500 \mathrm{~m}$. The depth corresponds to the isopycnal chosen by Reid (1981) to show the general patterns operating throughout the world oceans. The depth of this isopycnal rises to near the surface at high latitudes. Figure from Kroopnick (1985). 
ocean affects the preformed values of $\delta^{13} \mathrm{C}$ because the partial pressure of ${ }^{13} \mathrm{CO}_{2}$ in surface waters is not in equilibrium with that of the atmosphere.

The rate of gas exchange between the atmosphere and the ocean is such that it takes about a day to equilibrate the partial pressure of most gases with that of the atmosphere in a water column 3 meters high ("equilibrate" meaning that partial pressures in the ocean and the atmosphere are about equal). Therefore, it takes about 20 days for a 60 meter deep mixed layer to equilibrate with the atmosphere. This time scale would also be valid for carbon 13 if it were entirely in the form of dissolved ${ }^{13} \mathrm{CO}_{2}$. However, most of the carbon 13 (as well as most of the carbon 12) appears in the ocean in the form of bicarbonate ions $\mathrm{HCO}^{-}$. Dissolved $\mathrm{CO}_{2}$ converts to bicarbonate by the following reaction:

$$
\mathrm{H}_{2} \mathrm{O}+\mathrm{CO}_{2}+\mathrm{CO}_{3}^{2-} \Leftrightarrow 2 \mathrm{HCO}_{3}^{-}
$$

where $\mathrm{CO}_{2}$ designates dissolved $\mathrm{CO}_{2}$, and $\mathrm{CO}_{3}{ }^{2-}$ designates the carbonate ion. This reaction is very fast and can be considered to be instantaneous. Thus, if there is an imbalance between the ${ }^{13} \mathrm{C}$ partial pressure in the atmosphere and in the ocean, the concentration of ${ }^{13} \mathrm{CO}_{2}$ in the mixed layer begins to adjust; this adjustment is instantaneously buffered, however, because ${ }^{13} \mathrm{C}$ atoms present in $\mathrm{CO}_{2}$ are constantly exchanged with $\mathrm{HCO}_{3}$-. There is about 150 times more bicarbonate than dissolved $\mathrm{CO}_{2}$ in sea water, so it takes about 20 days $\mathrm{x} 150=3000$ days $\approx 10$ years for a 60 $\mathrm{m}$ deep mixed layer to adjust its ${ }^{13} \mathrm{C}$ partial pressure to that of the atmosphere. Therefore, surface waters cannot keep up with the seasonal cycle, and the $\delta^{13} \mathrm{C}$ of surface waters never equilibrates with that of the atmosphere. Thus, preformed values of $\delta^{13} \mathrm{C}$ also depend on how far from equilibrium regions of deep water formation are.

Another issue is that the $\delta^{13} \mathrm{C}$ of surface water in equilibrium with the atmosphere (hypothetical equilibrium) is strongly dependent on temperature. Different regions of formation of deep water have different temperatures, which contributes to different preformed values of $\delta^{13} \mathrm{C}$.

In summary, the various water masses are characterized by their preformed values of $\delta^{13} \mathrm{C}$. These preformed values depend both on the efficiency of the biology in high latitude surface waters and on air-sea interactions. The $\delta^{13} \mathrm{C}$ of deep water decreases as the water moves away from its source because of the remineralization of organic matter in the deep ocean. The Atlantic is 
flushed rapidly enough so that this remineralization is small compared to the effect of the mixing of Southern Ocean Waters and NADW.

\section{C - Data compilation}

In chapter $\mathrm{V}$, tracer data are used to constrain the deep circulation in an inverse model of the LGM Atlantic. Since $\delta^{13} \mathrm{C}$ and $\delta^{18} \mathrm{O}$ constitute the bulk of the data set, the study is limited to these isotopes. We keep in mind, however, that the results of the inverse model should be qualitatively consistent with the other data.

The $\delta^{13} \mathrm{C}$ and $\delta^{18} \mathrm{O}$ data set is compiled from published records, as well as from some unpublished data obtained from personal communications. This compilation is listed in appendix 1. All the measurements are made on Cibicidoides foraminifera. This species is thought to most reliably record the $\delta^{13} \mathrm{C}$ of total inorganic carbon dissolved in the deep ocean as well as the $\delta^{18} \mathrm{O}$ of sea water (with an offset due to temperature effects and a constant offset of $0.64 \%$ due to vital effects, see Mix, 1987, and section II-D-3). In the compilation, both $\delta^{13} \mathrm{C}$ and $\delta^{18} \mathrm{O}$ are referenced to the PDB standard (the standard ratio which appears in the $\delta$ notation, see section IIA-1). $\delta^{18} \mathrm{O}$ measurements referenced to the SMOW standard are converted to the PDB standard by adding $0.25 \%$ o to the SMOW values.

Note that there is an intercalibration problem between the different laboratories measuring $\delta^{18} \mathrm{O}$. This problem can create an offset of more than $0.1 \%$ o between different data sets (see Zahn and Mix, 1991). No systematic inter-laboratory calibration has been done yet, and it is not clear how to account for the offset. Therefore, the $\delta^{18} \mathrm{O}$ data are entered in the compilation with no correction; possible offsets are taken into account in the data error bars.

There are about a hundred $\delta^{18} \mathrm{O}$ and $\delta^{13} \mathrm{C}$ data points for the LGM in the North Atlantic (Figure II-C-1), but less than thirty data points in the South Atlantic. Most of the data come from the Eastern North Atlantic. Note that there are several data points for which $\delta^{18} \mathrm{O}$ measurements are published but $\delta^{13} \mathrm{C}$ measurements are not (or vice-versa). There are few data points from 

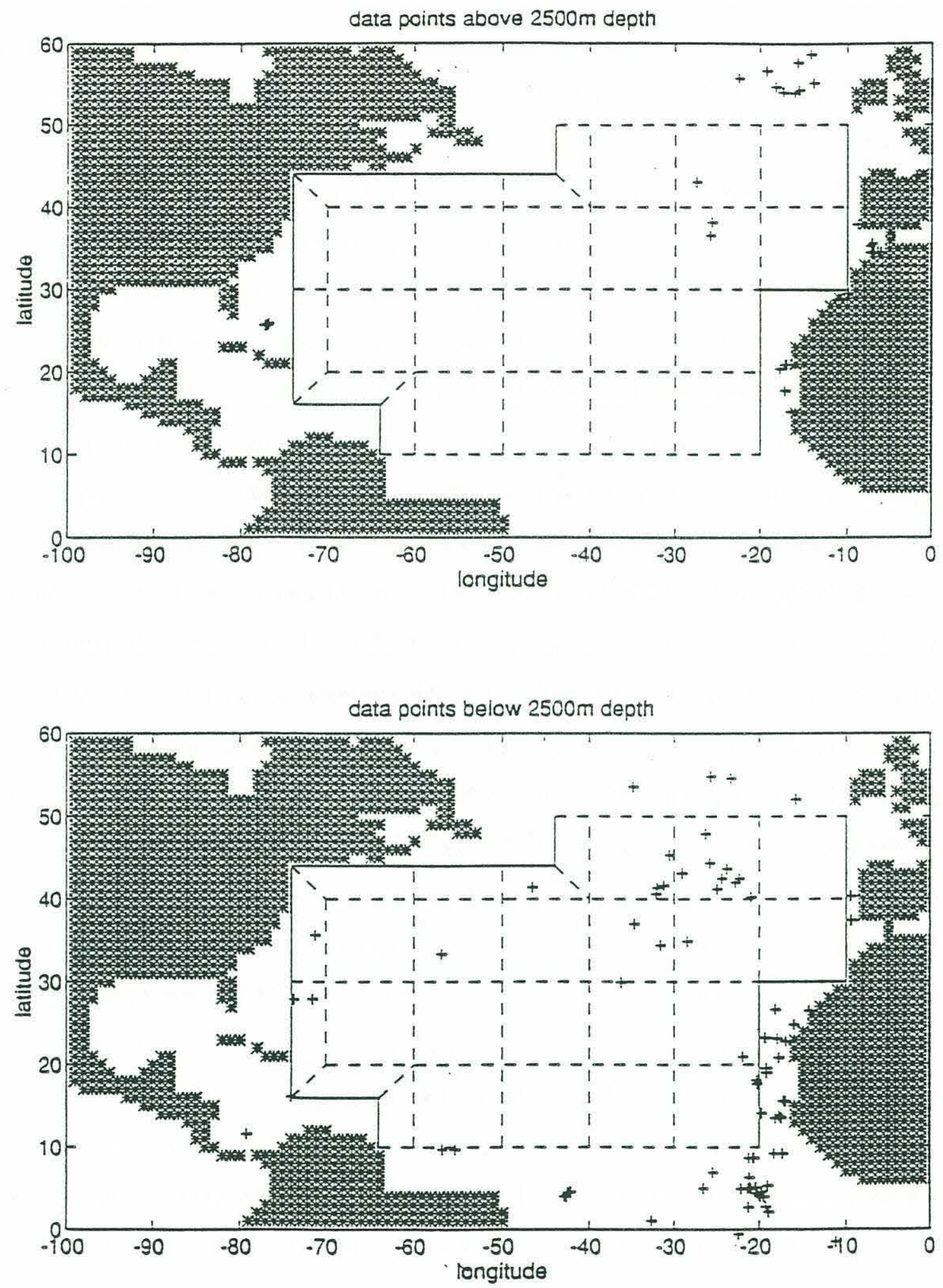

Figure II-C-1: Location of deep-sea sediment cores for which $\delta^{13} \mathrm{C}$ and $\delta^{18} \mathrm{O}$ LGM measurements are available. Top panel: sediment cores above $2500 \mathrm{~m}$ depth; bottom panel: sediment cores below $2500 \mathrm{~m}$ depth. 
intermediate layers of the ocean, partly because there are large areas of the North Atlantic where topography does not reach intermediate layers away from the continental margins. There are also few data points below $4.5 \mathrm{~km}$ depth in the North Atlantic because of calcium carbonate dissolution.

Figure II-C-2 shows a meridional section of $\delta^{13} \mathrm{C}$ in the interglacial Eastern Atlantic reconstructed from core-top foraminifera data. This figure is reconstructed by collapsing together all the data from the Eastern Atlantic onto a single section. This approach is not fully rigorous because it neglects the zonal gradients of $\delta^{13} \mathrm{C}$, but it is the only way to overcome the fact that benthic foraminifera data are available at the bottom of the ocean only. Foraminifera shells preserved in sediment core-tops grew during relatively recent times, and characterize the interglacial ocean. By comparison, Figure II-B-2 shows a Western Atlantic section, and part of an Eastern Atlantic section, of $\delta^{13} \mathrm{C}$ measured in sea water sampled during the GEOSECS expedition (Kroopnick, 1985). There are some discrepancies between the data sets such as the minimum observed in the $\delta^{13} \mathrm{C}$ values obtained from core-tops at about $4 \mathrm{~km}$ depth near the equator. No such minimum appears in the GEOSECS data. Also, high values in the top northern corner of the core-top data section (Figure II-C-2) are not matched in the Western Atlantic section of Kroopnick (Figure II-B-2, top panel). The differences may be due to the influence of Mediterranean water ( $\delta^{13} \mathrm{C}$-rich), which is much stronger in the Eastern Atlantic than in the Western Atlantic.

In general, the discrepancies are not very large (of the order of a few $0.1 \%$ ), and may be due to the fact that core-top data taken far apart are collapsed onto the same section. To confirm that these discrepancies are real, one would need measurements of $\delta^{13} \mathrm{C}$ in sea water just above the location of each sediment core. Very few of these measurements are available so far, but the most recent studies tend to measure $\delta^{13} \mathrm{C}$ values in the overlying water mass (see for instance Mackensen et al., 1993), and will help to better quantify the uncertainties associated with foraminifera data. Thus, in view of the available data, the $\delta^{13} \mathrm{C}$ distribution reconstructed from measurements in foraminifera shells appears to be consistent with measurements in sea water, and it seems reasonable to collapse foraminifera data onto meridional sections.

Figure II-C-3 suggests some similarity between the LGM water mass distribution and its modern counterpart (Figure II-C-2). In both reconstructions, the Atlantic is filled with $\delta^{13} \mathrm{C}$-rich 


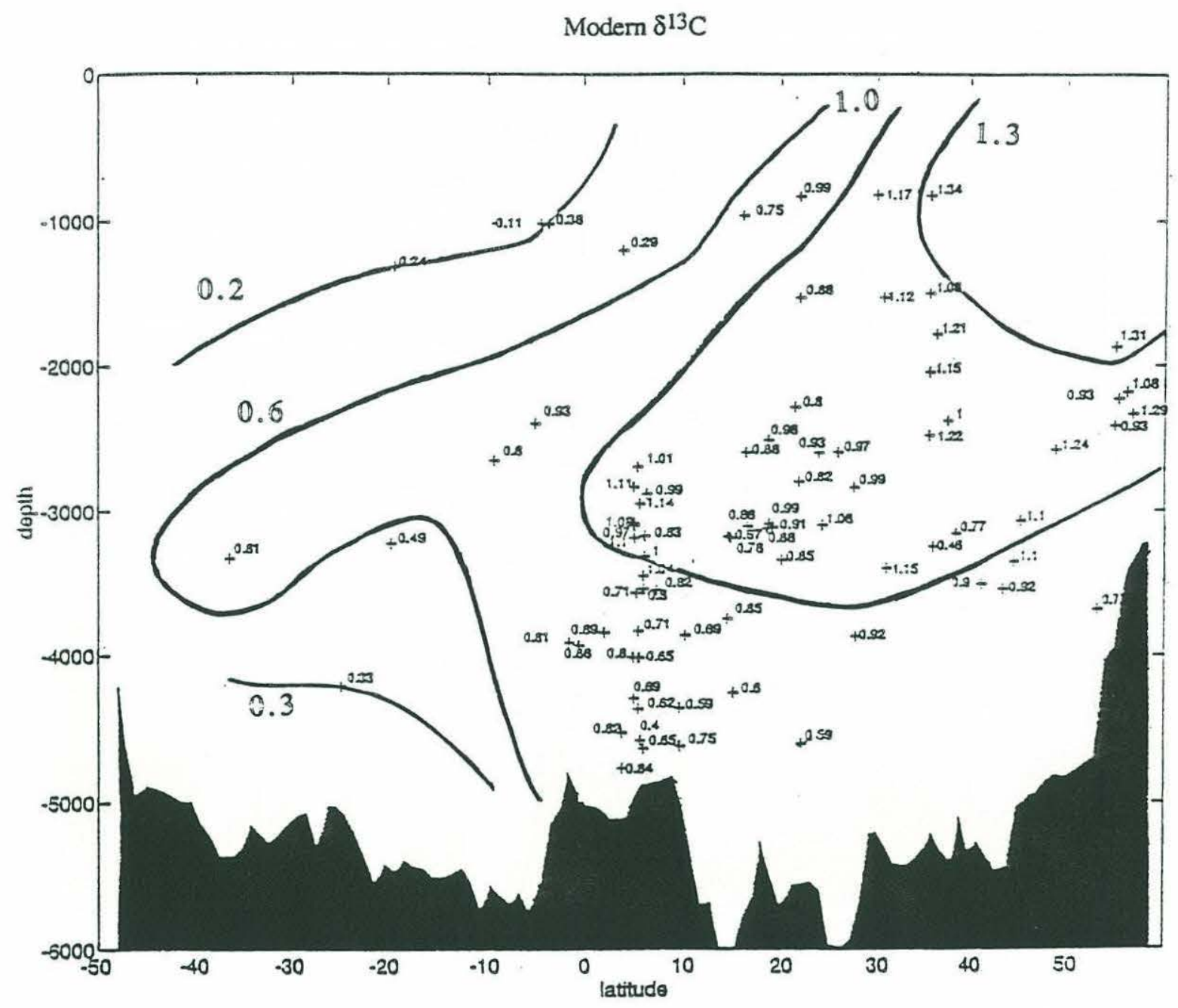

Figure II-C-2: Reconstruction of the latitudinal distribution of $8^{13} \mathrm{C}$ in the modern Eastern Atlantic. Data obtained from measurements in foraminifera shells preserved in sediment core-tops, see appendix 1 for provenance. Contours are hand-drawn. 
LGM $\delta^{13} \mathrm{C}$

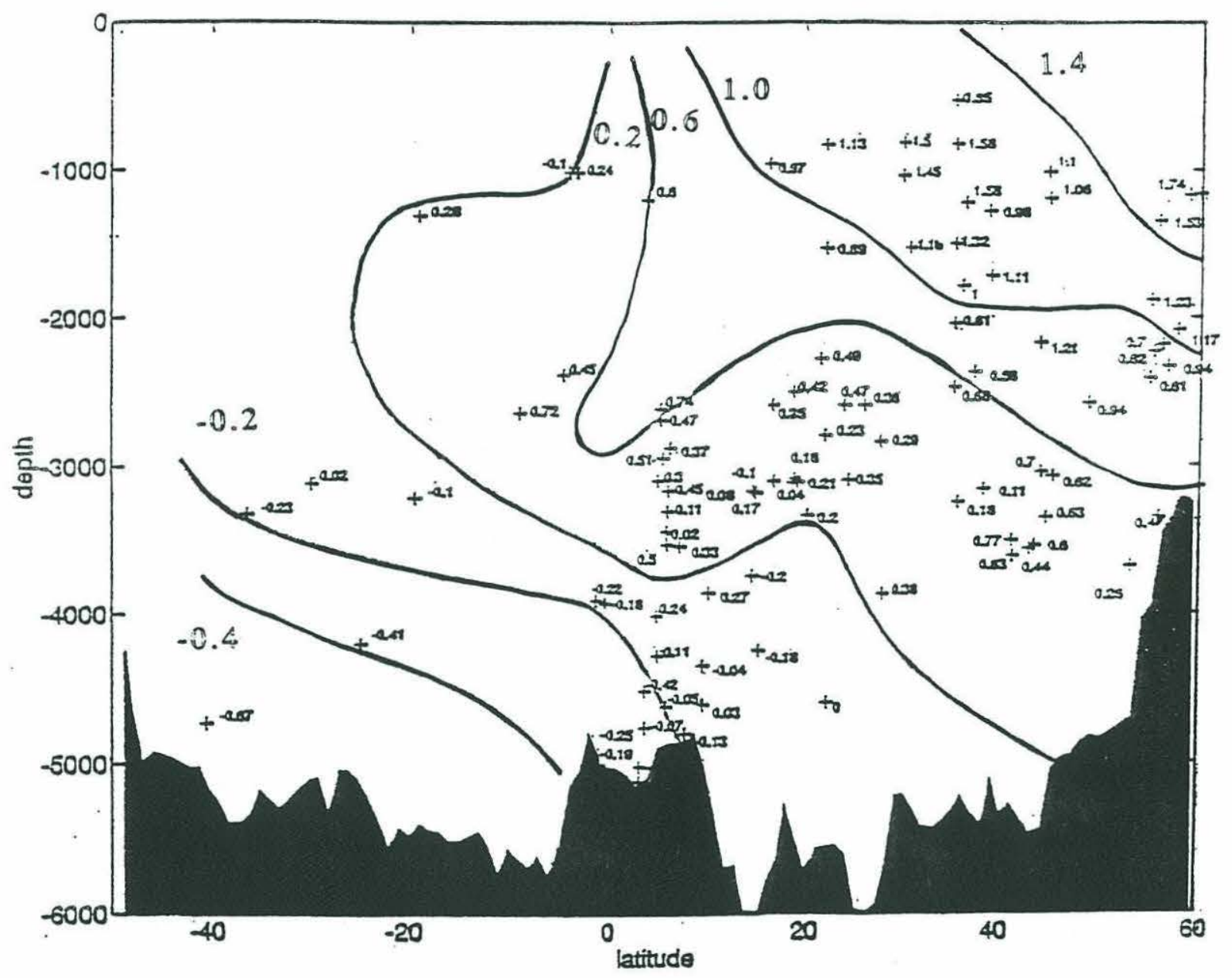

Figure II-C-3: Reconstruction of the latitudinal distribution of $\delta^{13} \mathrm{C}$ in the LGM Eastern Atlantic. Data obtained from measurements in foraminifera shells, see appendix 1 for provenance. Contours are hand-drawn. 
water (NADW in the modern ocean) overlying $\delta^{13} \mathrm{C}$-depleted water in the South Atlantic (AABW in the modern ocean). There is also some indication of the presence of $\delta^{13} \mathrm{C}$-depleted water in the intermediate layers of the glacial South Atlantic, which corresponds to AAIW in the modern ocean. This similarity suggests that one can use the same terminology to compare the water mass distribution in the glacial and in the modern ocean, and that it is not necessary to invoke the existence of radically different water masses.

Figure II-C-4 shows a section of the $\delta^{18} \mathrm{O}$ recorded in foraminifera shells. The $\delta^{18} \mathrm{O}$ signal recorded by foraminifera is significantly affected by temperature effects (see section II-D-3), and this section does not represent the $\delta^{18} \mathrm{O}$ of the water masses during the LGM.

Note that the choice of a contour interval of $0.4 \%$ in Figures II-C-2,3\&4 is deliberate 2 . Contouring the data with more finely spaced isolines (an isoline every $0.2 \%$ ) results in very complicated structures that are most probably data noise, even if the main outliers are removed. The fact that contours separated by at least $0.4 \%$ do not overlap suggests that the data have an error bar of about $\pm 0.2 \%$ on average. There are still some outliers when using the $0.4 \%$ o contour interval so this error bar is a rough standard deviation rather than an absolute bound on the data error. As discussed in part $\mathrm{B}$, the error bar is meant to represent the observational uncertainties due to bioturbation, fallout of organic matter, and to a lesser extent the limited analytical precision (as well as intercalibration problems for $\delta^{18} \mathrm{O}$ ). Individual error bars associated with each data point would allow a more realistic treatment of the observational uncertainties but are difficult to estimate. Experienced paleo-oceanographers can tell which data points are more reliable by looking at the stratigraphy of each sediment core, and thus produce a rough estimate of the observational uncertainty. Doing so for the entire available data set is beyond the scope of this study but should be done in the future in order to facilitate the use of paleo-data by modelers and in order to combine the data together in sections such as the ones shown in Figure II-C-2,3\&4.

\footnotetext{
2 All the contours in Figure II-C-2,3\&4 are hand-drawn.
} 


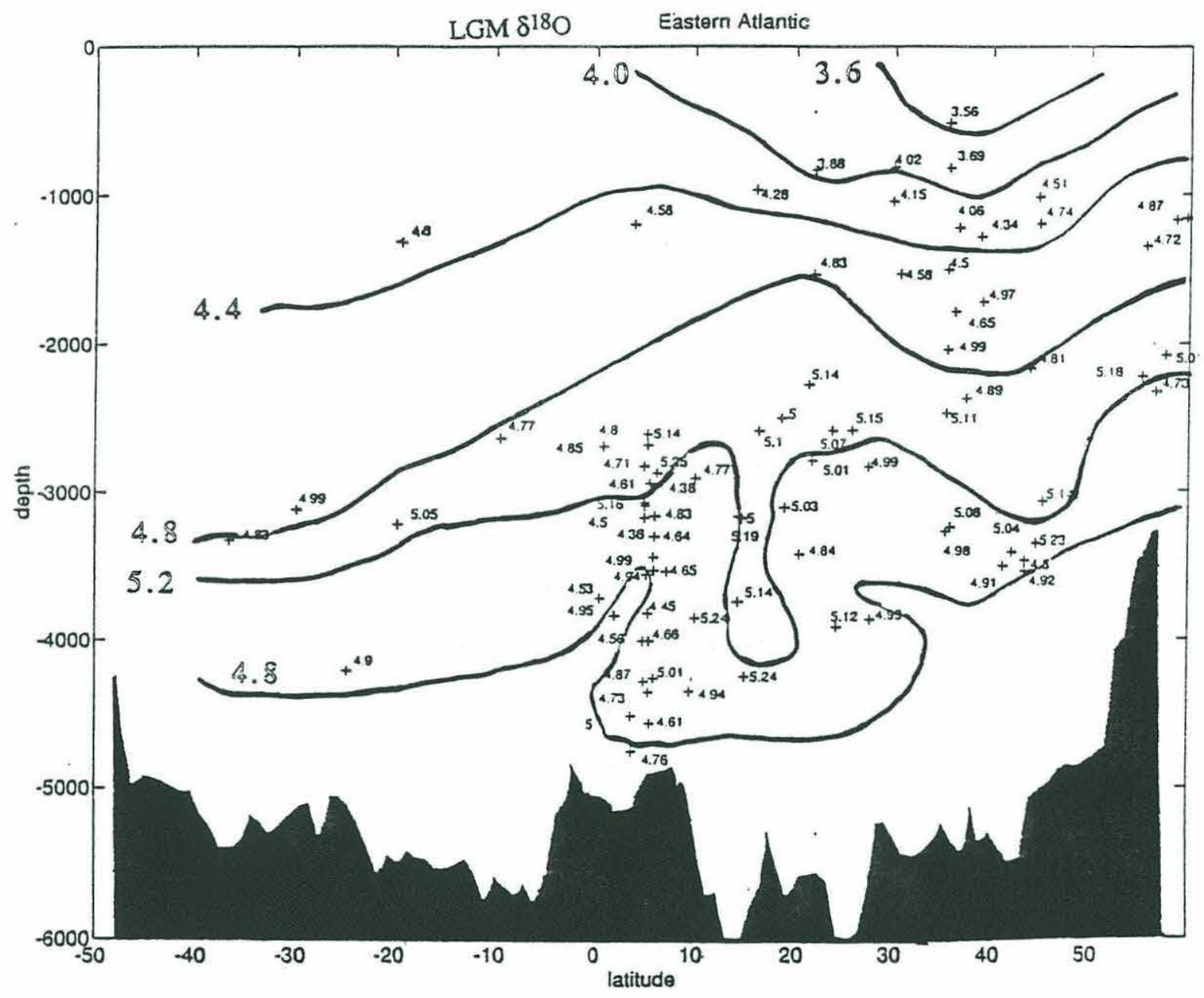

Figure II-C-4: Reconstruction of the latitudinal distribution of $\delta^{18} \mathrm{O}$ in the LGM Eastern Atlantic. Data obtained from measurements in foraminifera shells, see appendix 1 for provenance. Contours are hand-drawn. 


\section{D - Interpretation of the paleo-data in terms of changes in the water mass distribution}

In the modern Atlantic, the various water masses can be recognized in a meridional section of $\delta^{13} \mathrm{C}$ (Figure II-B-2, and Figure II-C-2). NADW is characterized by high preformed values of $\delta^{13} \mathrm{C}$ and waters from southern origins are characterized by low preformed values of $\delta^{13} \mathrm{C}$. The $\delta^{13} \mathrm{C}$ section reconstructed in part $\mathrm{C}$ of this chapter (Figure II-C-3) suggests that the same water masses were present in the Eastern Atlantic during the LGM.

It is tempting to compare the volumes of NADW and $\mathrm{AABW}$ in the modern ocean and in the glacial ocean, and see if they imply changes in the water mass distribution. Glacial preformed values of $\delta^{13} \mathrm{C}$ in NADW and in $\mathrm{AABW}$, however, are significantly different from their modern counterparts. We see here that preformed values in the glacial ocean are poorly determined, especially in the Southern Ocean, which renders the task of delimiting the various water masses difficult. Moreover, the contouring of the $\delta^{13} \mathrm{C}$ data is somewhat ambiguous, and different contouring suggest different water mass distributions.

Section 1 compares different reconstructions and their interpretation in terms of changes in the water mass distribution. The following sections examine the information provided by other paleotracers. Section 2 discusses the discrepancies existing between the $\delta^{13} \mathrm{C}$ data (a proxy for nutrients) and the Cadmium data (another proxy for nutrients). Section 3 describe the $\delta^{18} \mathrm{O}$ data, and their relation to oceanic temperatures. Finally, section 4 evaluates the potential of $\Delta^{14} \mathrm{C}$ data to constrain not only the distribution but also the rate of formation of the various water masses.

\section{1) Quantifying the changes in the water mass distribution}

The first attempt to contour a $\delta^{13} \mathrm{C}$ section in the LGM Eastern Atlantic is that of Duplessy et al. (1988, see Figure II-D-1). Their reconstruction suggests that the Last Ice Age water mass distribution is quite different from the modern ocean distribution. NADW is replaced by a small 


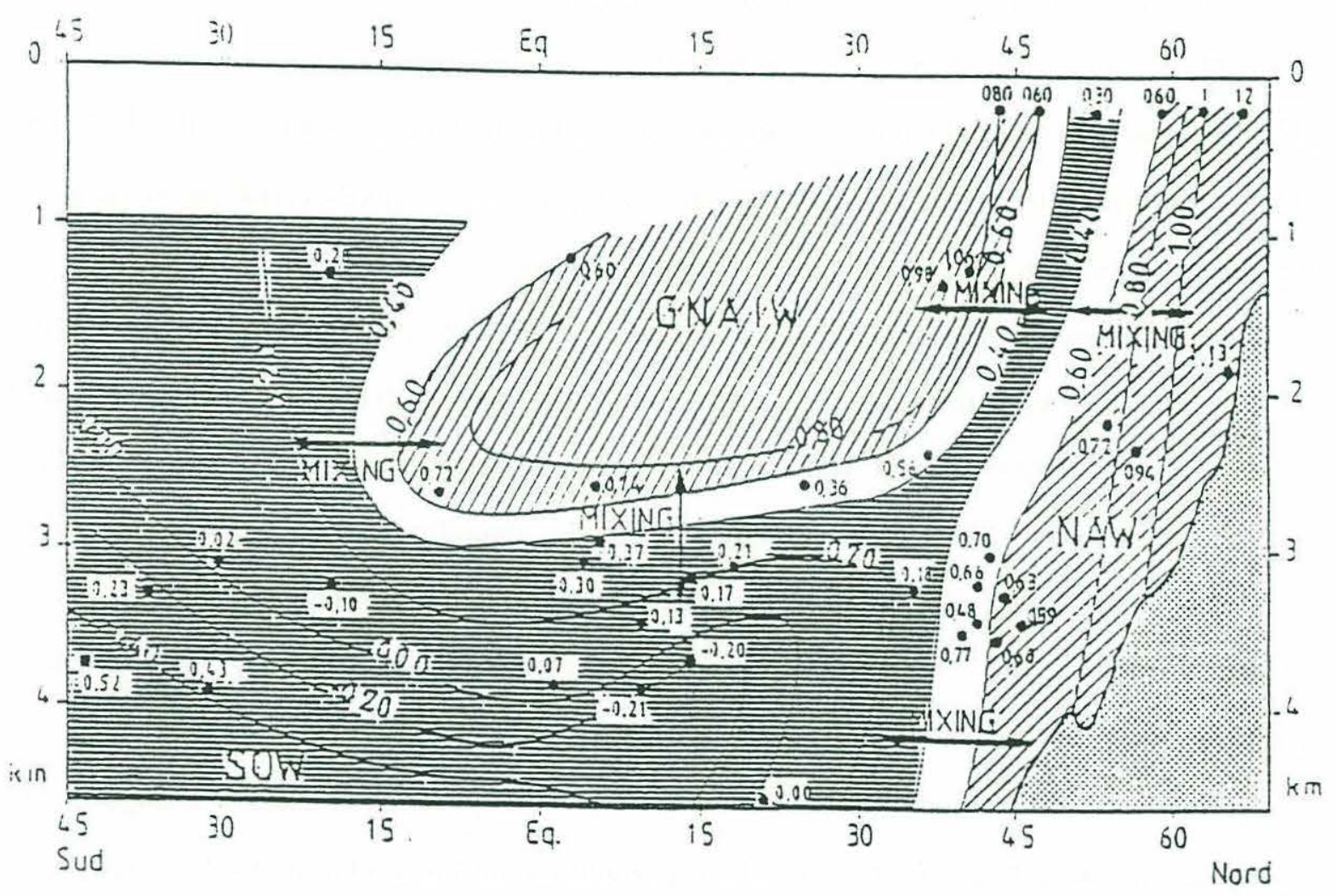

Figure II-D-1: Reconstruction of the latitudinal distribution of $8^{13} \mathrm{C}$ in the LGM Eastern Atlantic. The $\delta^{13} \mathrm{C}$ values of the total dissolved $\mathrm{CO}_{2}$ have been estimated from the $\delta^{13} \mathrm{C}$ value of benthic foraminifera genus Cibicides in 34 sediment cores. Surface $\delta^{13} \mathrm{C}$ values have been derived from those of $N$. Pachyderma using the correction factor of Labeyrie and Duplessy (1985). Figure from Duplessy et al. (1988). 
and shallow blob of $\delta^{13}$ C-rich water, which (following Boyle and Keigwin, 1987) they call Glacial North Atlantic Intermediate Water (GNAIW). The glacial deep Atlantic is filled with water of southern origin which they call Southern Ocean Water (SOW). GNAIW appears to be delimited by the $0.4 \%$ contour. This contour is closed by linking deep sea $\delta^{13} \mathrm{C}$ to surface water $\delta^{13} \mathrm{C}$. Doing so is not realistic because deep ocean water masses are separated from the surface by the thermocline and therefore decoupled from what happens in the upper ocean. Linking benthic data to planktonic data also creates very sharp fronts in the North Atlantic which are not plausible. Finally, more recent data at intermediate depths contradict the existence of such a blob of water (see Figure II-C-3).

Using the same data, Labeyrie et al. (1992) produce a more plausible reconstruction (see Figure II-D-2-b). The shading of the surfaces delimited by the $0.9 \%$ isolines in Figure II-D-2$\mathrm{a} \& \mathrm{~b}$, however, misleadingly leads the reader to compare the position of the $0.9 \%$ contour in the modern Atlantic and in the glacial Atlantic. In reality, the end-member composition of the waters formed in the North Atlantic is probably different in the glacial and the modern oceans, and the values of the isolines cannot be compared. Also, the almost vertical orientation of the $0.2 \%$, $0.6 \%$, and $0.9 \%$ contours in the northern section of Figure II-D-2-b is not required by the data, as shown in Figure II-C-3. In fact, no data could be found in the literature to constrain the Eastern Atlantic below $4 \mathrm{~km}$ depth, north of $20^{\circ} \mathrm{N}$. Note that the topography is different in Figure II-C-3 and in Figure II-D-2-b. The topography shown in Figure II-C-3 represents the maximum depth of the ocean in the Eastern Atlantic as a function of latitude. The topography shown in Figure II-D-2b probably represents the depth of the bottom as a function of latitude, along a fictitious cruise track in the Eastern Atlantic. An adaptation of Labeyrie et al's Figures, where the shading has been removed and the contours have been made more resembling to the contours of Figure II-C-3 in the northernmost region of the glacial Atlantic, is shown in Figures II-D-2-c\&d. As mentioned previously, significant differences between the Eastern and the Western Atlantic at shallow depths, north of $30^{\circ} \mathrm{N}$, are possible because of the stronger influence of waters of Mediterranean origins in the Eastern basin. Thus, the differences between the $\delta^{13} \mathrm{C}$ distributions in the top northern corners of the modern section (Figure II-B-2) and of the glacial section (Figure II-C-3) 

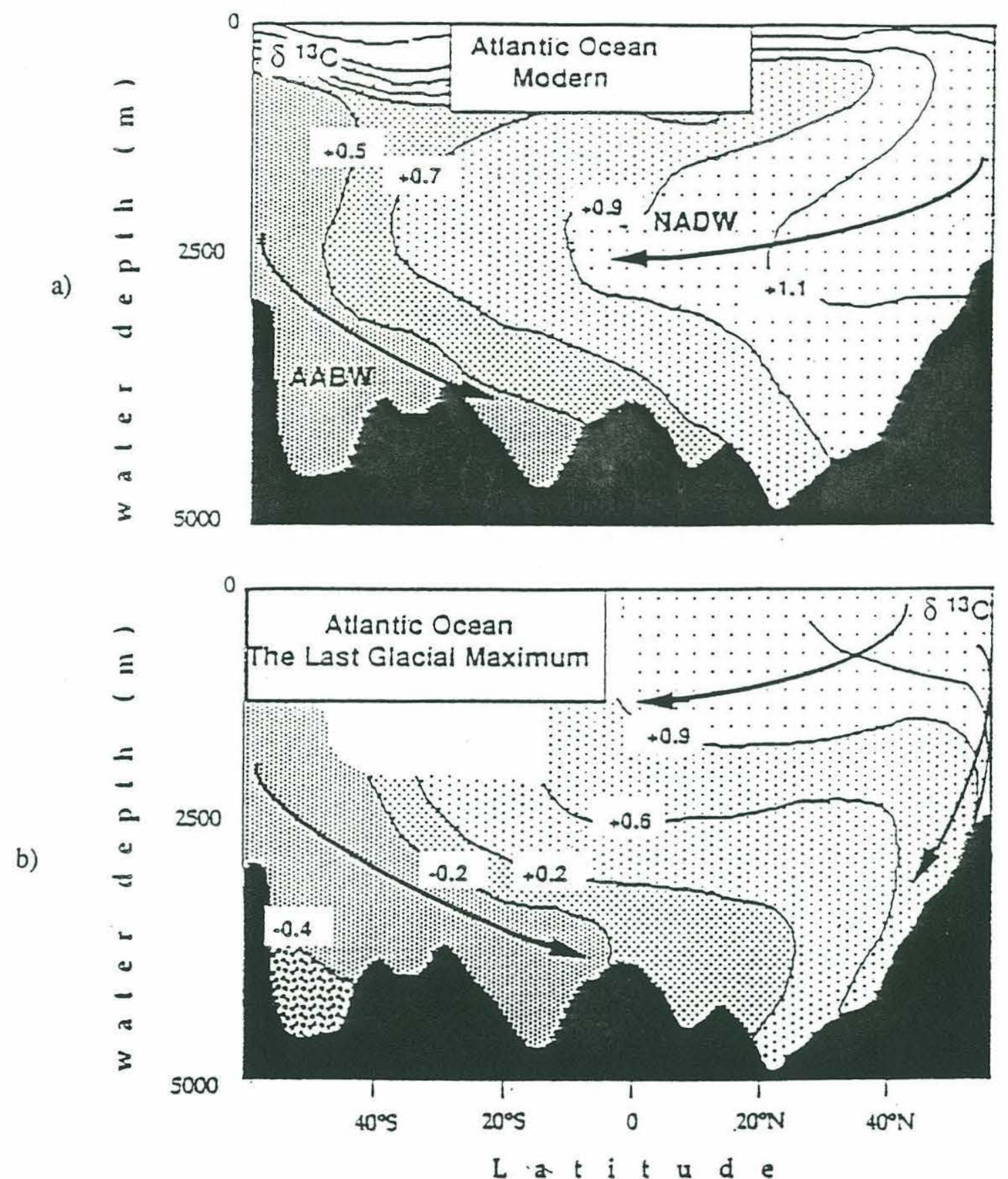

Figures II-D-2-a (top panel) \& II-D-2-b (bottom panel): Schematic comparison of the distribution of $\delta^{13} \mathrm{C}$ in the Atlantic during the modern period (II-D-2-a, adapted from Kroopnick, 1985) and during the glacial period (II-D-2-b, adapted from the $\delta^{13} \mathrm{C}$ distribution reconstructed by Duplessy et al. (1988) from measurements in benthic foraminifera). Figures from Labeyrie et al.(1992). 

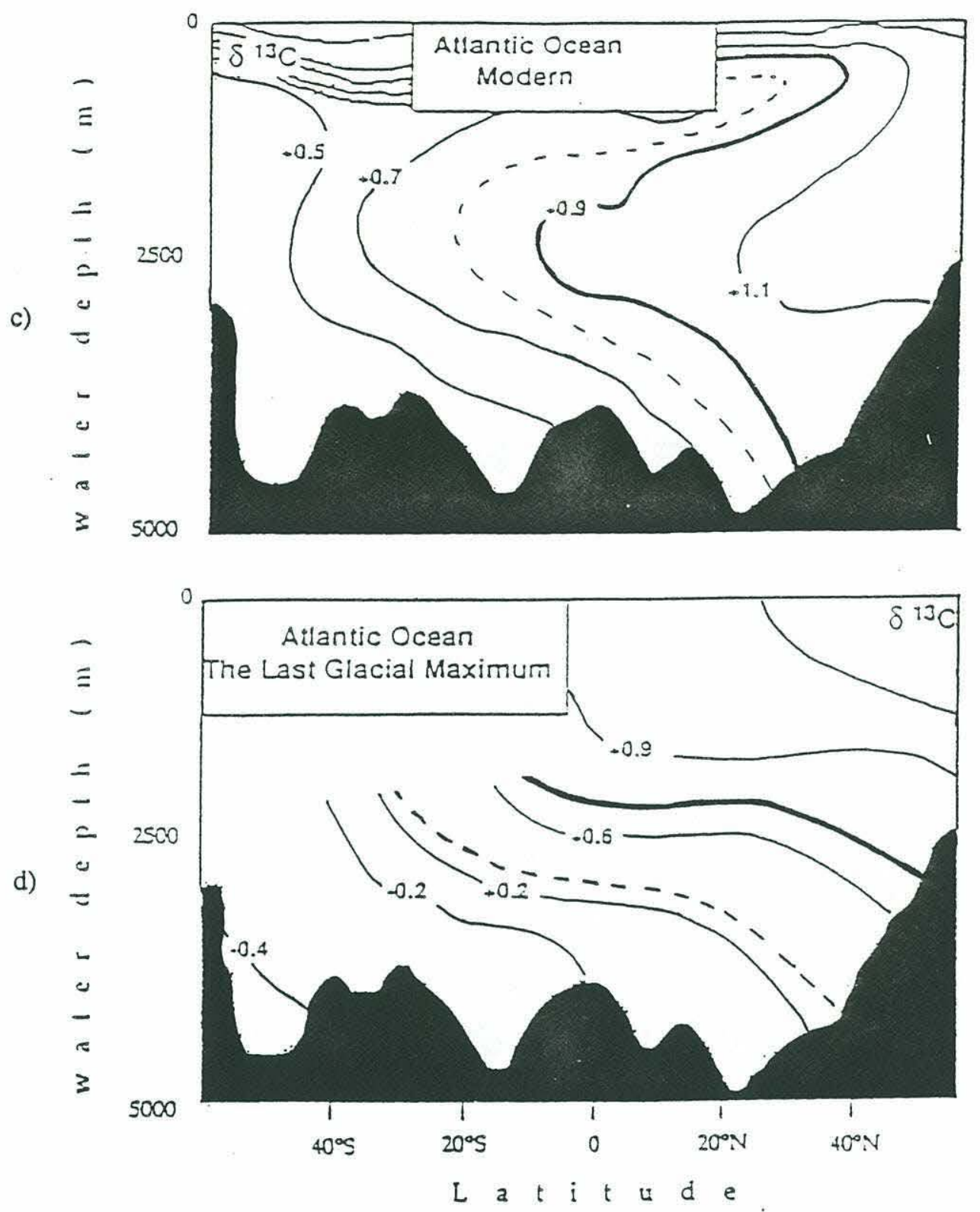

Figures II-D-2-c (top panel) \& II-D-2-d (bottom panel): Schematic comparison of the distribution of $\delta^{13} \mathrm{C}$ in the Atlantic during the modern period, as in Figure II-D-2-a\&b, except that the shading has been removed and the $0.2 \%, 0.6 \%$, and $0.9 \%$ contours have been redrawn in the northern section of the LGM reconstruction. Dashed lines indicate the position of waters composed of 50\% NADW and 50\% AABW $(0.8 \%$ contour in the interglacial section and $0.3 \%$ contour in the glacial ocean) for $A A B W$ end-member values of $0.5 \%$ in the interglacial Atlantic and $-0.85 \%$ in the glacial Atlantic, and for NADW end-member values of $1.1 \%$ in the interglacial Atlantic and $1.5 \%$ in the glacial Atlantic. Full lines indicate the position of waters composed of $2 / 3 \mathrm{NADW}$ and $1 / 3 \mathrm{AABW}(0.9 \%$ contour in the interglacial section and $0.7 \%$ contour in the glacial ocean) for the same end-member values. 

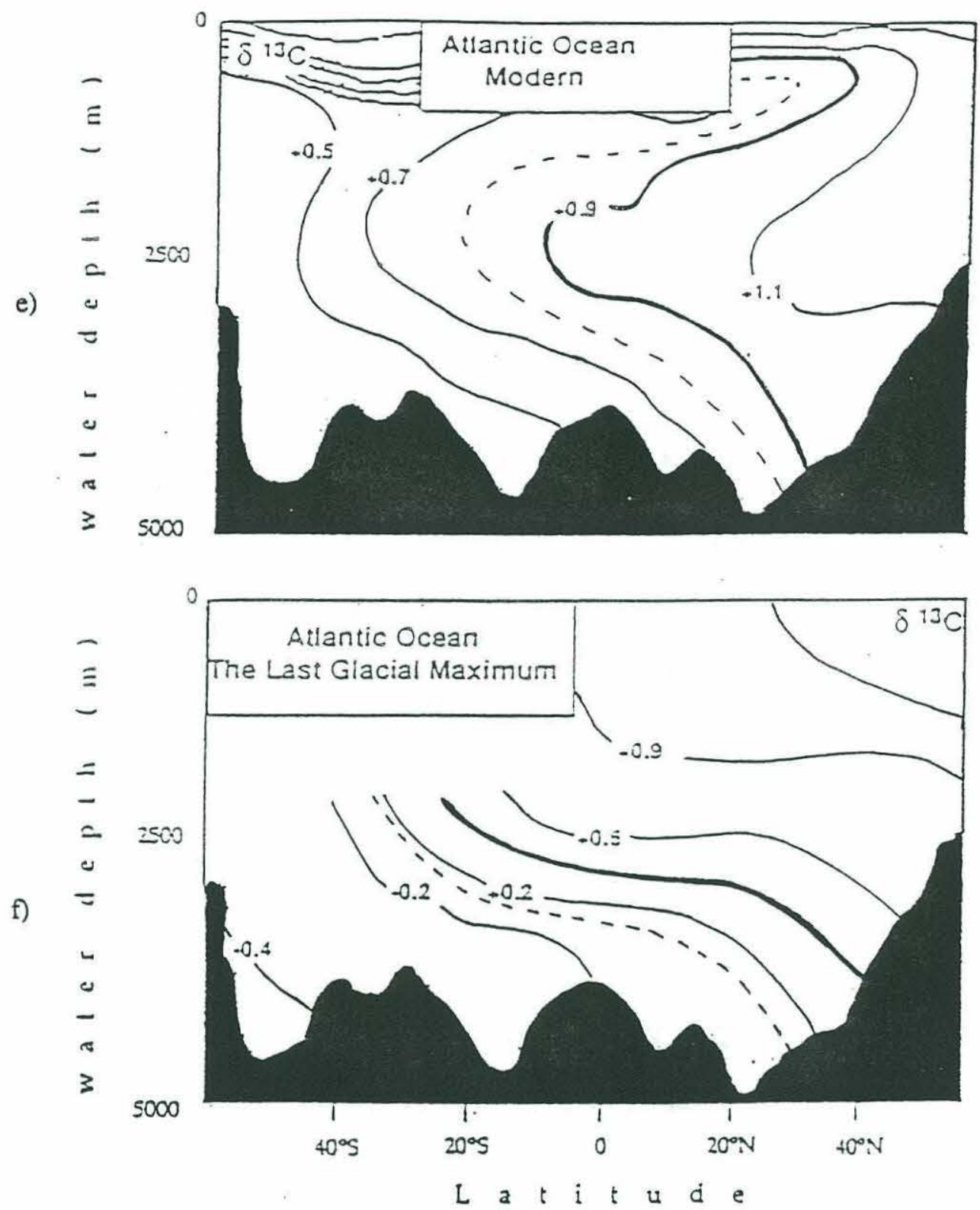

Figures II-D-2-e (top panel) \& II-D-2-f (bottom panel): Schematic comparison of the distribution of $\delta^{13} \mathrm{C}$ in the Atlantic during the modern period, as in Figure II-D-2-a\&b, except that the shading has been removed and the $0.2 \%, 0.6 \%$, and $0.9 \% c$ contours have been redrawn in the northern section of the LGM reconstruction. Dashed lines indicate the position of waters composed of 50\% NADW and $50 \%$ AABW ( $0.8 \%$ contour in the interglacial section and $0.1 \%$ contour in the glacial ocean) for $A A B W$ end-member values of $0.5 \%$ in the interglacial Atlantic and $-0.4 \%$ in the glacial Atlantic, and for NADW endmember values of $1.1 \%$ in the interglacial Atlantic and $1.1 \%$ in the glacial Atlantic. Full lines indicate the position of waters composed of $2 / 3$ NADW and $1 / 3$ AABW $(0.9 \%$ contour in the interglacial section and $0.4 \%$ contour in the glacial ocein) for the same end-member values. 
cannot be interpreted as temporal changes, because these two sections are meant to represent opposite sides of the Atlantic.

An approach to delimiting the various water masses in the Atlantic is to consider the tracer distribution in the deep ocean as a mixing process between the AABW end-member and the NADW end-member (Broecker et al., 1991). For the glacial ocean, it seems that a reasonable value for the southern end-member is $-0.85 \%$ (Charles and Fairbanks, 1992) and a reasonable value for the northern end-member is 1.5\%o (Oppo and Lehmann, 1993). A parcel of water made of $50 \%$ of each end-member has a $\delta^{13} \mathrm{C}$ of:

$$
(1.5-0.85) \div 2 \approx 0.3 \% \text { o }
$$

For the modern ocean, it seems that a reasonable value for the southern end-member is $0.5 \%$ o and a reasonable value for the northern end-member is $1.1 \%$ (Figure II-D-2-a). Thus, in the modern ocean, a mixture of $50 \%$ of each end-member has a $\delta^{13} \mathrm{C}$ of:

$$
(1.1+0.5)+2=0.8 \%
$$

There are only slight differences between the positions of the $50 \%$ lines defined by the $0.3 \%$ o contour in the glacial ocean, and by the $0.8 \%$ contour in the modern ocean (drawn as dashed lines in Figures II-D-2-c\&d). If one defines NADW as the water mass north of the $50 \%$ line, one obtains comparable volumes of NADW in the glacial and in the modern oceans.

One gets a different picture when comparing the position of the isolines delimiting waters composed of more than $2 / 3 \mathrm{NADW}$. With the same end-member values as above, the $2 / 3 \mathrm{NADW}$ $+1 / 3$ AABW mixture is delimited by the $0.7 \%$ line in the glacial Atlantic and the $0.9 \%$ line in the modern Atlantic (drawn as thicker lines in Figures II-D-2-c\&d). The volume located north the $0.7 \%$ line in the LGM section (Figure II-D-2-d) is shallower than the volume located north the $0.9 \%$ line in the modern section (Figure II-D-2-c), which suggests a reduced volume of Lower NADW during glacial times.

$\delta^{13} \mathrm{C}$ values as high as $1.5 \%$ are not found below 1500 meter depth in the LGM Atlantic (see Figure II-C-3; see also Oppo and Lehman, 1993), and hence one may argue that they are significantly affected by waters from the thermocline, which are rich in $\delta^{13} \mathrm{C}$ (see Slowey and Curry, 1992). In this case, the "true" value of the northern end-member may be lower than $1.5 \%$. Assuming that this "true" value is as low as the modern northern end-member, $1.1 \%$, one finds in 
the LGM ocean that the $50 \%$ line is defined by the $0.1 \%$ o contour, and that the line delimiting the $2 / 3 \mathrm{NADW}+1 / 3 \mathrm{AABW}$ mixture is defined by the $0.4 \%$ o contour. These two contours are drawn as a dashed line and a thick line in Figure II-D-2-f. The volumes delimited by these lines in Figure II-D-2-f (glacial) are only slightly shallower than the corresponding volumes in Figure II-D-2-e (interglacial), which suggests that the volume of Lower NADW was only slightly reduced during the LGM, compared to the modern situation.

The uncertainty in the end-member composition may also result in an under-estimation of the reduction of the volume of Lower NADW. Indeed, the value of $-0.85 \%$ o for the southern endmember in the LGM Atlantic may be too negative (Mackensen et al., 1993; see next section also). If instead one takes a value of $-0.4 \%$ o to characterize this end-member and a value of $1.5 \%$ to characterize the northern end-member, one obtains a value of $0.5 \%$ o for the $50 \%$ mixture, and a value of $0.9 \%$ for the $2 / 3 \mathrm{NADW}+1 / 3 \mathrm{AABW}$ mixture. The isolines delimiting waters containing more than $50 \%$ of NADW or more than $2 / 3$ of NADW are thus shallower than in the first example (with end-members of $-0.85 \%$ and $1.5 \%$ ), and significantly shallower than in the modern ocean (compare the position of the $0.9 \%$ isoline in figure II-D-2-c with the position of the $0.9 \%$ isoline in figure II-D-2-d). These relatively high end-member values therefore correspond to a much reduced volume of Lower NADW in the glacial ocean. The higher the end-member values are, the smaller the volume of Lower NADW is.

Thus, delimiting the water masses with tracer isolines corresponding to various mixtures of the end-members does not suffice to determine the water mass distribution, because the endmembers are not known well enough. It seems that the volume of Lower NADW was reduced during the LGM, but the extent of this reduction cannot be quantified.

Note that the water mass distribution is even less constrained in the Western Atlantic because of the scarcity of data there. A recent reconstruction by Lohmann and Lohmann (1994) suggests a $\delta^{13} \mathrm{C}$ distribution in the glacial ocean different from that of the modern ocean (Figure II-D-3). Their $\delta^{13} \mathrm{C}$ section implies a vertical stratification of $\delta^{13} \mathrm{C}$ in the glacial Western Atlantic. This vertical stratification is mainly constrained by a vertical profile located at the Rio Grande Rise 


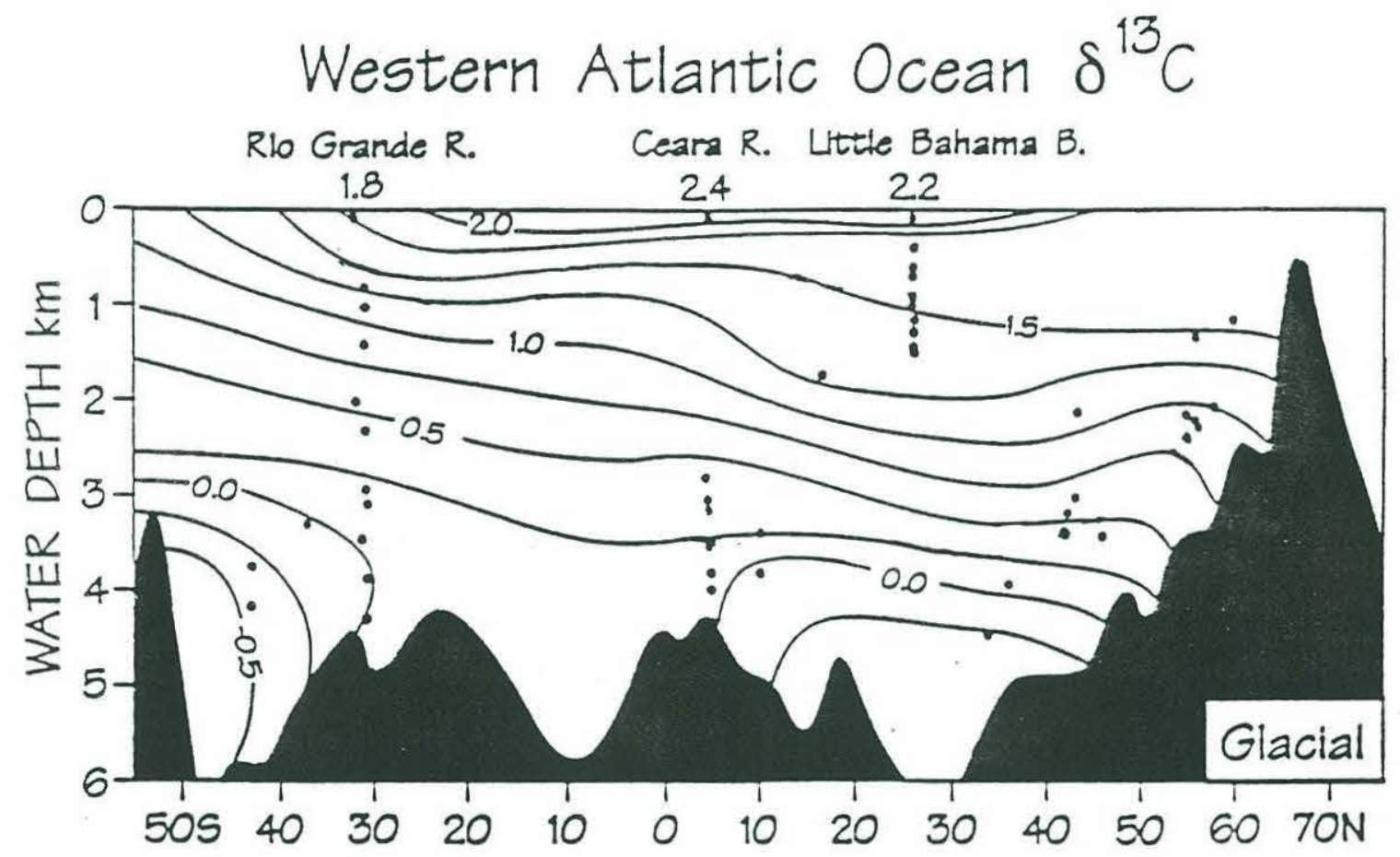

Figure II-D-3: Reconstruction of the latitudinal distribution of $\delta^{13} \mathrm{C}$ in the LGM Western Atlantic. Figure from Lohmann and Lohmann (1994). 
(Figure II-D-5; Lohmann and Lohmann, 1994). This low productivity region should not be influenced by fallouts of organic matter. A comparison of water column measurements and core top data in the modern ocean shows a very good agreement (Lohmann and Lohmann, 1994; see Figure II-D-4) which gives some confidence in the reliability of this data set.

The glacial Western Atlantic reconstruction appears quite different from the various glacial Eastern Atlantic reconstructions, especially in the South Atlantic. A large difference between the tracer concentration in Eastern and Western basins is plausible below the top of the Mid-Atlantic Ridge as shown in a zonal section of $\mathrm{PO}_{4}$ in the modern Atlantic, at $15^{\circ} \mathrm{S}$ (Figure II-D-6). However, the large difference in glacial $\delta^{13} \mathrm{C}$ values above the Mid-Atlantic Ridge (about $1 \%$ ) implied by the foraminifera data is improbable. Note that upper Eastern Atlantic data (3 data points) come from a high productivity region off the African coast, and may be too low because of fallout of organic carbon. In fact, a meridional section of $\delta^{13} \mathrm{C}$ in the Eastern Atlantic very similar to that of Lohmann and Lohmann (1994) can be reconstructed by simply removing these three data points (Figure II-D-7).

In summary, several different reconstructions of the meridional distribution of $\delta^{13} \mathrm{C}$ are possible in the LGM Atlantic. The interpretation of $\delta^{13} \mathrm{C}$ data in terms of water mass distribution is quite ambiguous. This ambiguity is due to the scarcity of data in the Deep North Atlantic and in the Southern and Western Atlantic, and the lack of information on the end-members. Depending on the criteria chosen to define the water masses, one finds that the water mass distribution during the LGM was moderately or was noticeably different from the modern ocean.

A final remark is that the fact that deep waters are depleted in $\delta^{13} \mathrm{C}$, and intermediate waters are enriched in $\delta^{13} \mathrm{C}$, as was the case in the glacial Atlantic, does not necessarily mean that the water mass distribution has changed. It can simply be due to a change in the preformed values of $\delta^{13} \mathrm{C}$ which characterize the end-members. Preformed values of $\delta^{13} \mathrm{C}$ in $\mathrm{AABW}$ seem to have been lower, and preformed values of $\delta^{13} \mathrm{C}$ in NADW seem to have been higher during the LGM than during interglacial times. This difference, by itself, can create $\delta^{13} \mathrm{C}$ depleted deep waters and $\delta^{13} \mathrm{C}$ enriched intermediate waters in the North Atlantic, and must explain part of the signal observed in the glacial ocean. 


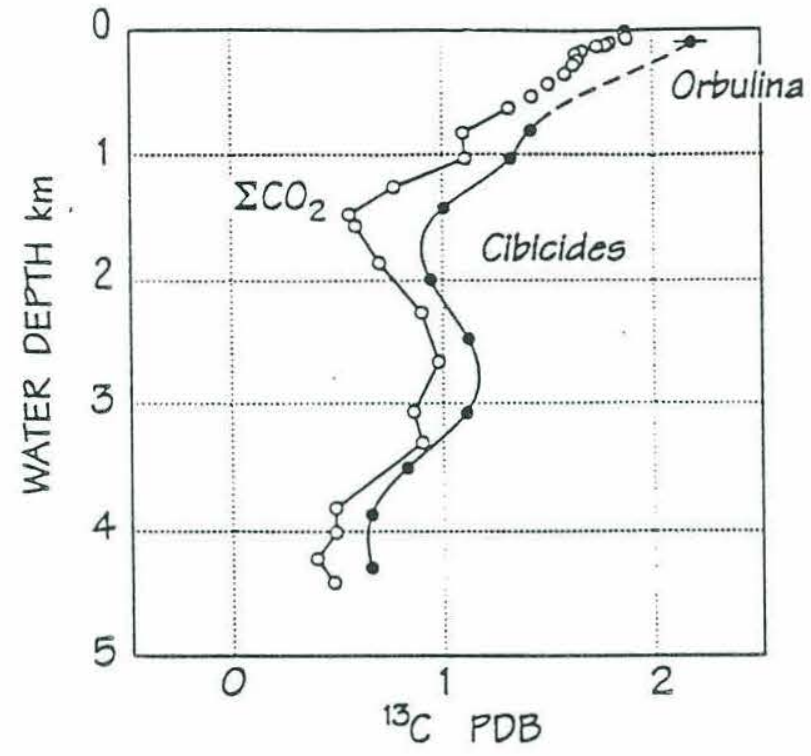

Figure II-D-4: Comparison of core-top data (full circles) and sea-water measurements in the modern ocean (open circles) at the Rio Grande Rise. The rise is located off the South American coast, at about $30^{\circ} \mathrm{S}$. The good agreement between the two data sets suggests that the core-top data are reliable despite the low sedimentation rate near the Rio Grande Rise. Figure from Lohmann and Lohmann (1994).

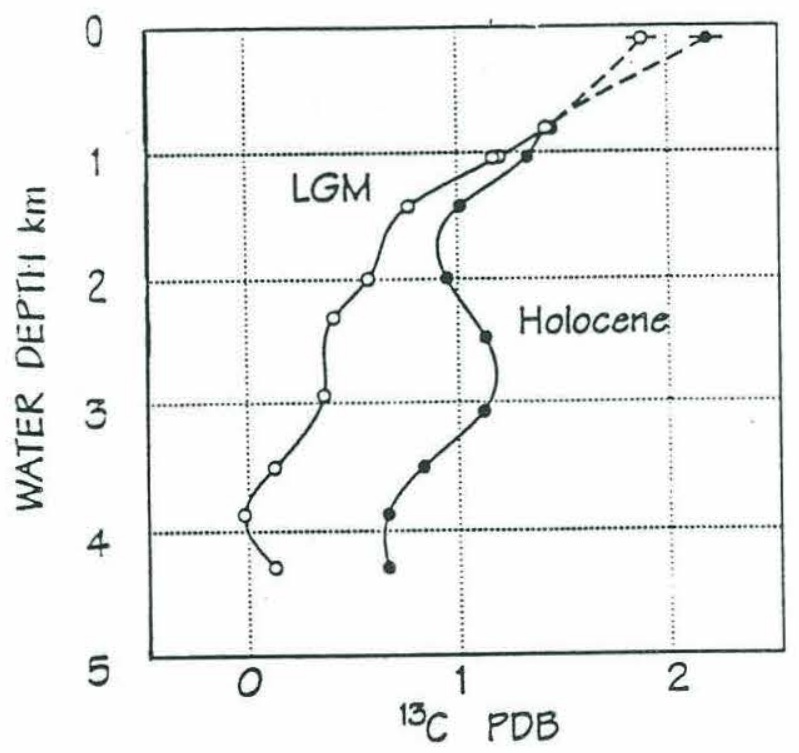

Figure II-D-5: Modern (Holocene) and glacial vertical profiles of $\delta^{13} \mathrm{C}$ at the Rio Grande Rise. The rise is located off the South American coast, at about $30^{\circ} \mathrm{S}$. Figure from Lohmann and Lohmann (1994). 


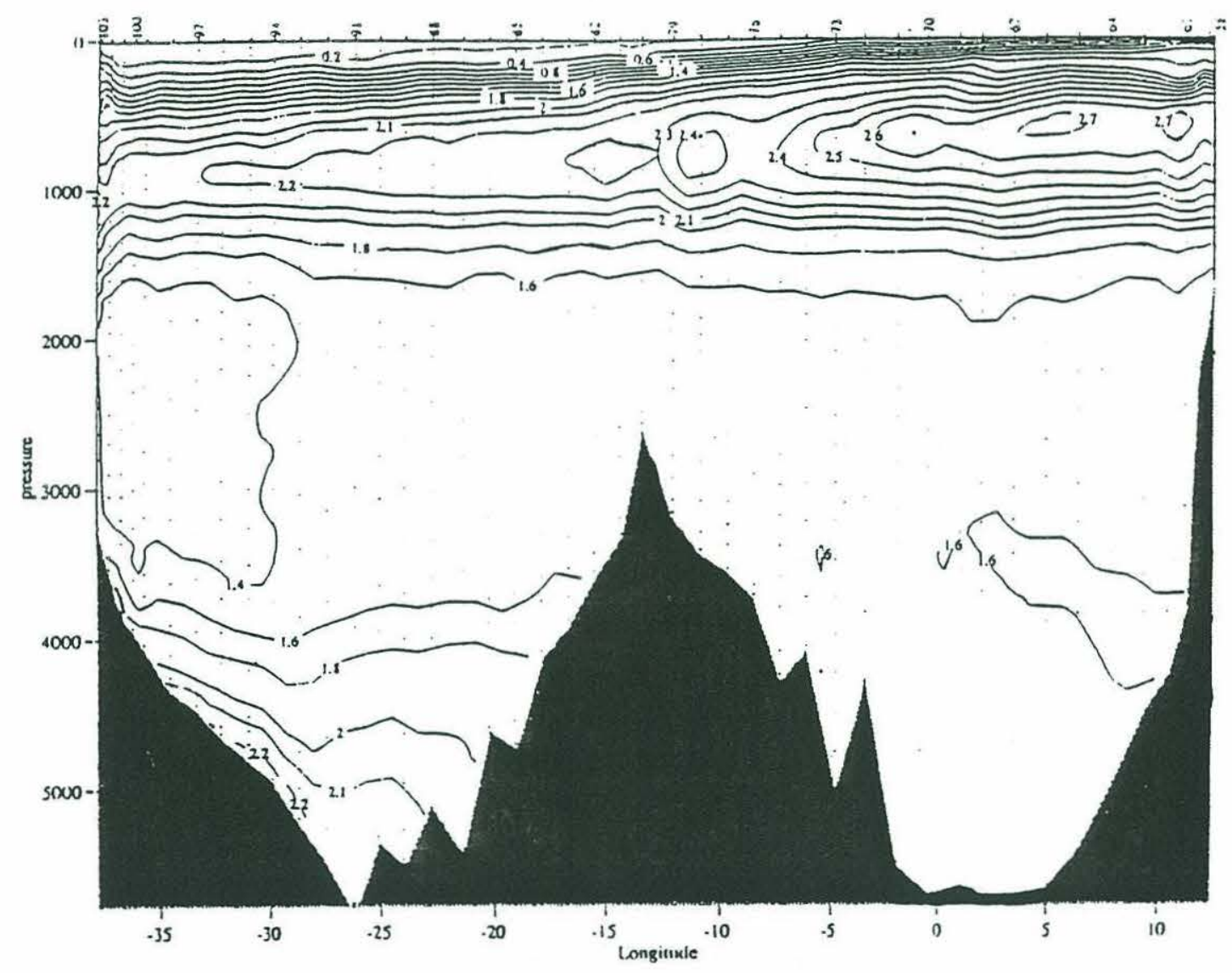

Figure II-D-6: Zonal section of phosphorus, at $15^{\circ} \mathrm{S}$. Nutrient gradients are quite small in the modern ocean at intermediate depths (below $1 \mathrm{~km}$ ). It is not clear whether the large difference between the $\delta^{13} \mathrm{C}$ values in the Eastern and in the Western Atlantic during the LGM at these same depths is plausible. Figure drawn by P. Robbins, Woods Hole Oceanographic Institution, using data from the South Atlantic Ventilation Experiment. 


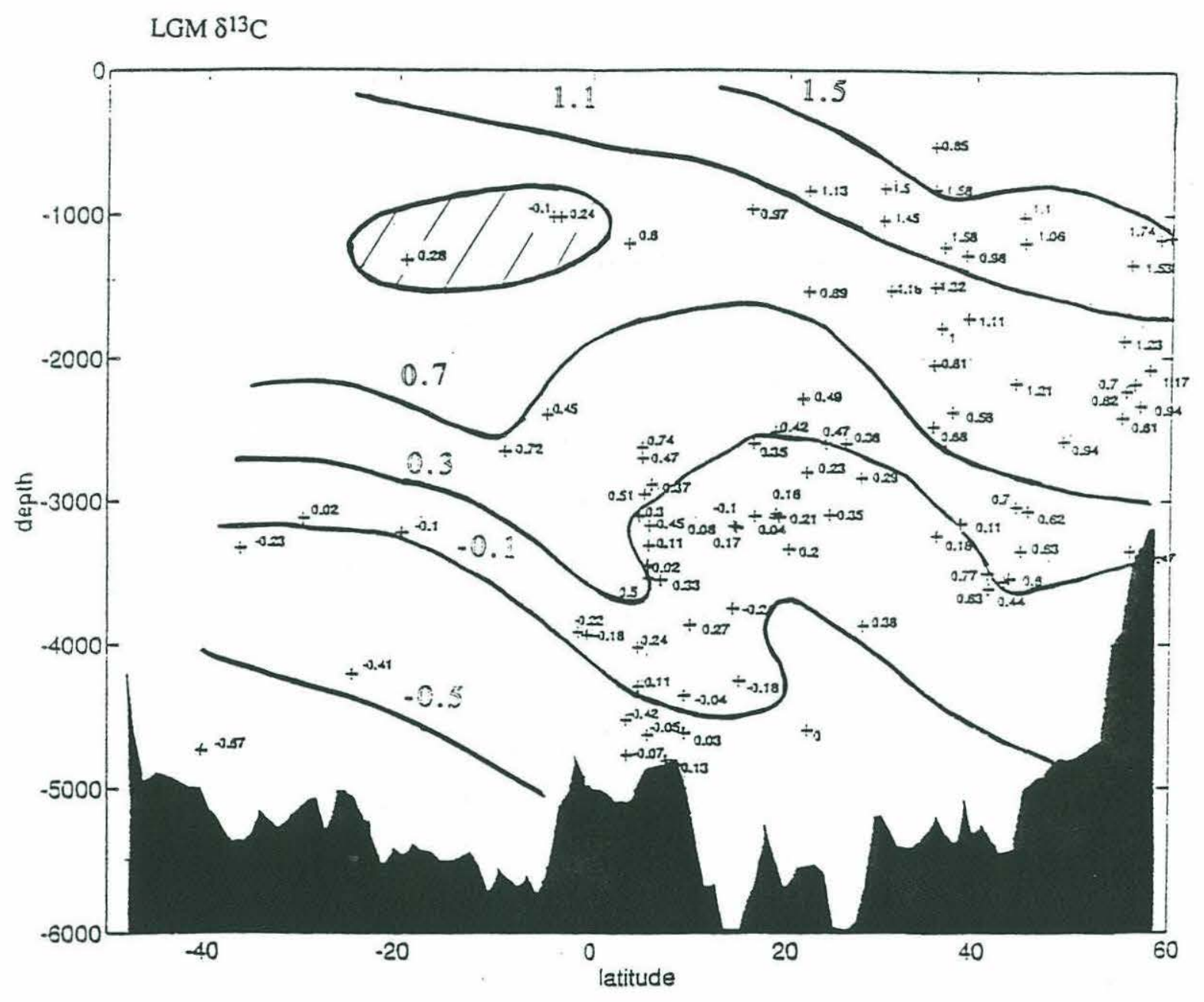

Figure II-D-7: Reconstruction of the latitudinal distribution of $8^{13} \mathrm{C}$ in the LGM Easterm Atlantic. Same data as in Figure II-C-3, except that the three data points in the hatched area. which seem to be inconsistent with Lohmann and Lohmann's data points (1994), are not taken into account. Contours are hand-drawn. This reconstruction is quite similar to that of Lohmann and Lohmann (1994) shown in figure II-D-3. 


\section{2) Discrepancies between $\delta 13 \mathrm{C}$ data and Cadmium data}

In the modern ocean, the cadmium concentration is proportional to the phosphorus concentration and is strongly correlated with $\delta^{13} \mathrm{C}$. There are some discrepancies, however, between $\delta^{13} \mathrm{C}$ and $\mathrm{Cd}$ data in the LGM ocean (see Boyle, 1992, for an extensive discussion of this problem). The main discrepancy is that $\delta^{13} \mathrm{C}$ data imply that, on a global average, the upper waters of the ocean were depleted in nutrients during the LGM and that the nutrient maximum observed at mid-depths in the modern ocean was shifted to the deep layers. In contrast, Cd data do not show such a major redistribution of nutrients in the glacial world ocean. Similarly, $\delta^{13} \mathrm{C}$ data indicate that the deep waters of the Southern Ocean were as enriched (or even more enriched) in nutrients as anywhere in the glacial ocean, whereas Cd data suggest that the LGM nutrient levels were about the same as the present levels in the Southern Ocean. These discrepancies do not mean that one or both tracers are misleading. $\delta^{13} \mathrm{C}$ and $\mathrm{Cd}$ could behave differently, for example because preformed values of $\delta^{13} \mathrm{C}$ are affected by air-sea gas exchange but preformed values of $\mathrm{Cd}$ are not affected ${ }^{3}$. Doubts have been expressed about the reliability of $\delta^{13} \mathrm{C}$ measurements in the Circumpolar Current (Mackensen et al., 1993). Comparison of core top data and samples of bottom sea water suggests that the $\delta^{13} \mathrm{C}$ recorded by foraminifera is depleted compared to that of the overlying water by as much as $0.9 \%$ o near polar fronts. Indeed, polar fronts are high

productivity regions, so significant amounts of $\delta^{13} \mathrm{C}$ depleted organic materials can reach the sediments in these areas, and lower the $\delta^{13} \mathrm{C}$ of the water in which benthic foraminifera grow.

As mentioned in the previous section, the interpretation of $\delta^{13} \mathrm{C}$ data in terms of water mass distribution will remain ambiguous until the values of $\delta^{13} \mathrm{C}$ characterizing the southern endmember is better constrained and the discrepancies between $\delta^{13} \mathrm{C}$ and $\mathrm{Cd}$ data are solved.

\section{3) $\delta 180$ and temperature effects}

\footnotetext{
${ }^{3}$ There is essentially no cadmium in the atmosphere.
} 
The use of a second tracer would help to better characterize the various water masses. There are about as many $\delta^{18} \mathrm{O}$ data points and $\delta^{13} \mathrm{C}$ data points, so one could hope to construct $\delta^{13} \mathrm{C}$ $\delta^{18} \mathrm{O}$ diagrams similar to the T-S diagrams used in the modern ocean. The interpretation of $\delta^{18} \mathrm{O}$ data in terms of water masses, however, is rendered difficult because of temperature effects. The $\delta^{18} \mathrm{O}$ recorded in the calcium carbonate of foraminifera shells cannot be directly used as a tracer of the water masses because it is influenced by sea water temperature. The temperature fractionation effect can be described by the following relation:

$$
\delta^{18} \mathrm{O}_{\mathrm{c}}=\delta^{18} \mathrm{O}_{\mathrm{W}}+\mathrm{f}(\mathrm{T})
$$

where $\delta^{18} \mathrm{O}_{\mathrm{C}}$ is the value recorded by benthic foraminifera, $\delta^{18} \mathrm{O}_{\mathrm{W}}$ is the $\delta^{18} \mathrm{O}$ of the sea water in which foraminifera grow, and $f(T)$ is a known function of sea water temperature. $f(T)$ has been empirically determined in the deep ocean by Shackleton (1974; see also the review by Mix, 1987) for temperatures less than $16^{\circ} \mathrm{C}$ :

$$
\mathrm{T}=\mathrm{a}+\mathrm{b}\left(\delta^{18} \mathrm{O}_{\mathrm{c}}-\delta^{18} \mathrm{O}_{\mathrm{W}}\right)
$$

with $\mathrm{a}=16.9$ and $\mathrm{b}=-4.0\left(\mathrm{~T}\right.$ in ${ }^{\circ} \mathrm{C}, \delta^{18} \mathrm{O}$ in $\%$ ). Note that $\delta^{18} \mathrm{O}_{\mathrm{w}}$ and $\delta^{18} \mathrm{O}_{\mathrm{c}}$ must be referenced to the same standard (PDB or SMOW). Moreover, this relation is valid for the Uvigerina species only. The Cibicidoides species does not grow in thermodynamic equilibrium with sea water so its $\delta^{18} \mathrm{O}_{\mathrm{c}}$ must be corrected:

$$
\delta^{18} \mathrm{O}_{\mathrm{c}}=\delta^{18} \mathrm{O}_{\text {cibicidoides }}+0.64 \% \text { o }
$$

The $0.64 \%$ correction has also been determined empirically.

Ideally, one would like to separate the contribution of temperature to $\delta^{18} \mathrm{O}_{\mathrm{c}}$ from that of $\delta^{18} \mathrm{O}_{\mathrm{W}}$. Temperature gradients dominate the $\delta^{18} \mathrm{O}_{\mathrm{c}}$ signal in the modern ocean $(\mathrm{Zahn}$ and Mix, 1991 ), and probably did so during the LGM. However, $\delta^{18} \mathrm{O}_{\mathrm{w}}$ gradients are not negligible.

Separating the two contributions in the $\delta^{18} \mathrm{O}_{\mathrm{c}}$ of planktonic foraminifera is feasible thanks to the CLIMAP SST reconstruction, which gives $\mathrm{f}(\mathrm{T})$. The other contribution, $\delta^{18} \mathrm{O}_{\mathrm{W}}$, is composed of a term which depends on the volume of ice stored in continental glaciers (calculated for the LGM using paleo-sea level reconstructions) and a term characteristic of each water mass (set by the evaporation-precipitation cycle):

$$
\delta^{18} \mathrm{O}_{\mathrm{W}}=\delta^{18} \mathrm{O}_{\mathrm{g}}+\delta^{18} \mathrm{O}_{\mathrm{wat} . \text { char. }}
$$

$\delta^{18} \mathrm{O}_{\mathrm{wat} . c h a r}$ is a tracer of the water masses, and can be calculated using the following relation: 


$$
\delta^{18} \mathrm{O}_{\text {wat.char. }}=\delta^{18} \mathrm{O}_{\mathrm{c}}-\mathrm{f}(\mathrm{T})-\delta^{18} \mathrm{Og}_{\mathrm{g}}
$$

$\delta^{18} \mathrm{O}_{\mathrm{C}}$ is measured in foraminifera shells; $\mathrm{f}(\mathrm{T})$ is known if SST is known; $\delta^{18} \mathrm{Og}$ is also quite well known. Note that $\delta^{18} \mathrm{O}_{\text {wat.char. }}$ is the small difference of large numbers so we expect this estimate to be quite noisy ${ }^{4}$.

As yet, there is no way of removing the temperature signal from $\delta^{18} \mathrm{O}_{\mathrm{c}}$ data in the deep ocean.

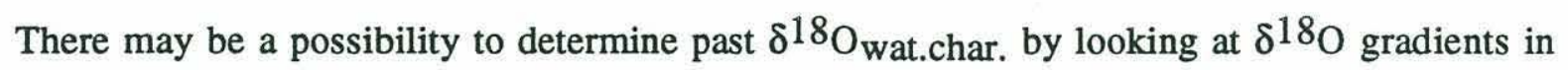
sediment pore waters (Schrag and DePaolo, 1993) but there are only a few such measurements 5 . However, one could at least use $\delta^{18} \mathrm{O}_{\text {wat.char. as a tracer of the water masses and estimate deep }}$ sea temperatures by subtracting $\delta^{18} \mathrm{O}_{\mathrm{w}}$ from $\delta^{18} \mathrm{O}_{\mathrm{c}}$.

In summary, the direct interpretation of $\delta^{18} \mathrm{O}$ measurements in benthic foraminifera as a tracer of the water masses is not possible because of temperature effects; deep sea temperature cannot be estimated independently of $\delta^{18} \mathrm{O}_{\mathrm{c}}$. The fact that a direct interpretation of the $\delta^{18} \mathrm{O}$ data in terms of water mass distribution is not yet possible, does not mean that these data are useless. They are used in models of the Glacial Atlantic (see chapter V) because they provide some weak constraints on the temperature field.

\section{4) Carbon 14 data}

They are a few measurements of $\Delta^{14} \mathrm{C}$ in benthic and planktonic foraminifera in the Equatorial Atlantic for the LGM. They indicate that the ${ }^{14} \mathrm{C}$ age difference between deep and surface waters was larger during the Last Ice Age than today, and that the age difference between mid-depth and

\footnotetext{
${ }^{4}$ Note that, in the modern upper ocean, there is a strong correlation between $\delta^{18} \mathrm{O}_{\text {wat.char. and salinity because }}$ they are both determined by the evaporation-precipitation cycle. Duplessy et al. (1991) use this correlation to reconstruct sea surface salinities during the LGM. This reconstruction could provide interesting information about the water column stratification in regions of deep water formation but it is still imprecise because of uncertainties on $\delta^{18} \mathrm{O}_{\text {wat.char. }}$. It is not sure that the modern $\delta^{18} \mathrm{O}_{\text {wat.char. } / \text { salinity relation was valid during the Last Ice }}$ Age, but model results indicate that this might have been the case (Joussaume et al., 1984). In any case, if one really wants to learn about the water column stratification, one needs to reconstruct salinity and temperature in the deep ocean as well, which is much more difficult.

5 Even if this approach works, it will still be impossible to reconstruct paleo-salinities in the deep ocean because the $\delta^{18} \mathrm{O}_{\text {wat.char. }}$ / salinity relation in the deep ocean depends on the water mass characteristics which were probably different during the LGM.
} 
surface waters was smaller (Broecker et al., 1990). These data are interpreted as evidence for a greater southward extent of "young" NADW (or GNAIW) in the mid-depth equatorial Atlantic, and a greater northward extent of "old" $\mathrm{AABW}$ (SOW) in the deep equatorial Atlantic. However, there are several reasons why these data are not conclusive yet.

First, there are only 4 data points published and they are all located in the Equatorial Atlantic. Like $\delta^{13} \mathrm{C}$, preformed values of $\Delta^{14} \mathrm{C}$ can vary because of different air-sea gas exchange or different biological activity. The fact that the deep water age relative to the surface waters goes from 350 years today to 675 years during the LGM could be simply due to older preformed values of ${ }^{14} \mathrm{C}$ in the NADW. Moreover, the Knorr 110-50GGC core, used by Broecker et al. to determine the water mass distribution in the glacial Atlantic, is located at $4 \mathrm{~km}$ depth, $4^{\circ} \mathrm{N}$, which is a zone of transition between waters of southern origins and waters of northern origins in the modern ocean. Therefore, changes recorded in the Knorr 110-50GGC core could be due to small displacements of this zone of transition. As in the case of $\delta^{13} \mathrm{C}$, determining the extent of the volume of $\mathrm{AABW}$ will require more data.

Another issue is that it is necessary to have measurements both in benthic and planktonic foraminifera in order to separate the water age signal from that of the foraminifera shells themselves (these shells are about 15000 years old, so most of the carbon 14 signal is due to the age of the shells, not the age of the water mass). In order to compare the two types of foraminifera shells, one has to insure that they grew at the same time. Because of bioturbation, shells picked in the same layer of sediments can have different ages, especially in low accumulation rate cores. To overcome this difficulty, it is necessary to pick about a thousand shells of each type of foraminifera so that planktonic and benthic samples have about the same age after averaging. Because $\Delta^{14} \mathrm{C}$ ages are the small difference of two large numbers, we expect the data to be noisy.

Reconstructing the position of the water masses during the LGM is not the best application of the $\Delta^{14} \mathrm{C}$ data. The same work can be done using $\delta^{13} \mathrm{C}$ data which are much cheaper to obtain. The main potential of $\Delta^{14} \mathrm{C}$ data is the capability to date the water masses, and thus their capacity to directly constrain the rate of ventilation of the deep ocean. Schematically, the age difference between deep and surface waters is the sum of the preformed age of the deep water mass and of the aging term due to radioactive decay of ${ }^{14} \mathrm{C}$ after the deep water mass has been formed. When 
$\Delta^{14} \mathrm{C}$ data are available close to the regions of formation of deep water, it will be possible to remove the contribution of preformed ages to the benthic-planktonic age difference and to estimate the aging term (for such a study in the modern ocean, see Broecker et al., 1991). Because changes in the ventilation rate of the deep ocean result in changes in the aging term, $\Delta^{14} \mathrm{C}$ will provide direct constraints on the rate of formation of deep waters during the LGM.

\section{Summary}

We have seen in this chapter that the distribution of several geochemical tracers can be reconstructed in the Last Ice Age ocean from the analysis of foraminifera shells. The most widely studied tracers are $\delta^{18} \mathrm{O}$ which is linked to the ocean temperature, and $\delta^{13} \mathrm{C}$ which is closely related to the nutrient cycle. The North Atlantic is by far the best documented ocean with more than a hundred data points. Using $\delta^{13} \mathrm{C}$ data, it is possible to make some inferences about the distribution of the various water masses in the Glacial Atlantic Ocean. There is some ambiguity however, because of the paucity of data in the Western and in the Southern Atlantic and in the bottom layers of the North Atlantic. An analysis of the $\delta^{13} \mathrm{C}$ data in terms of mixing between two end-members does not remove the ambiguity because the composition of the end-members are poorly constrained. Using a $\delta^{13} \mathrm{C}$ end-member value of $-0.85 \%$ o for glacial $\mathrm{AABW}$ and a $\delta^{13} \mathrm{C}$ end-member value of $1.1 \%$ for glacial NADW, and a meridional section of $\delta^{13} \mathrm{C}$ adapted from Labeyrie et al. (1992), one finds a water mass distribution in the glacial Atlantic quite similar to the water mass distribution of the modern Atlantic, with a volume of Lower NADW only slightly reduced in the glacial ocean. The values of $\delta^{13} \mathrm{C}$ are different because the composition of the endmembers is different in the glacial ocean and in the modern ocean. More specifically, during the LGM low preformed $\delta^{13} \mathrm{C}$ values in the source of $\mathrm{AABW}$ tend to produce low $\delta^{13} \mathrm{C}$ values in the entire deep Atlantic. The low $\delta^{13} \mathrm{C}$ values observed in the glacial North Atlantic can be partly explained by this process.

Using higher $\delta^{13} \mathrm{C}$ end-member values, one finds a significantly reduced volume of NADW in the glacial ocean. Thus, the extent to which the glacial water mass distribution is different from the modern ocean is poorly constrained. The reduction of the volume of Lower NADW in the 
glacial ocean proposed by Boyle and Keigwin (1987), Duplessy et al. (1988), and Labeyrie et al. (1992) has yet to be quantified.

More $\delta^{13} \mathrm{C}$ data are needed in the regions of formation of deep water to improve our knowledge of the glacial ocean.

The following chapters are concerned with the interpretation of the paleo-data in terms of circulation changes in the glacial Atlantic Ocean. Boyle and Keigwin (1987) and Labeyrie et al. (1992) argue that a significantly reduced volume of Lower NADW indicates a reduction in the rate of formation of Lower NADW during the Last Ice Age. The paleo-data are reinterpreted in chapter $\mathrm{V}$ using an inverse model of the oceanic circulation. The $\delta^{13} \mathrm{C}$ value of the southern end-member is assumed to be about $-0.4 \%$ o during the LGM. This relatively high value results in a significantly reduced volume of Lower NADW, similar to what is proposed by Boyle and Keigwin (1987) and Labeyrie et al. (1992). The objective of the inverse model simulations is to verify whether a change in the water mass distribution is sufficient to determine the ventilation of the Atlantic during the LGM. Before analyzing the model results, we examine in chapter III the optimization procedure used to assimilate the paleoceanographic data in the model, and in chapter IV the model itself. 


\section{Chapter III - Combining physical and observational constraints: an optimization problem.}

A problem, not specific to paleoceanography, is to combine observations and known physics of the ocean. Observations are often scarce and noisy, and one would like to improve knowledge of the ocean by taking into account physical constraints as well as observational ones. In the simulations presented in chapter $\mathrm{V}$, physical constraints appear as equations in numerical models of the ocean circulation. These equations are simplified representations of the real ocean, and they should not be strictly imposed. Similarly, observations are subject to observational uncertainties, and cannot be taken at face value. This chapter describes an approach to combine observational and physical constraints which finds the best fit between data and model equations. The notion of best fit is associated with the minimum of a cost (or objective) function. The best-fit solution usually does not satisfy the model equations exactly, and does not reproduce the observations perfectly. It is however, the best compromise between the two sets of constraints.

The cost functions used in this work usually appear as the sum of a data misfit term and a model equation imbalance term. The role of the data misfit term is to ensure that the model results are consistent with the observations within the observational uncertainties. In the paleoceanographic simulations presented in chapter V, the observations consist of geochemical tracer data such as carbon 13 and oxygen 18. The observational uncertainties associated with paleo-data are difficult to evaluate, but these uncertainties are necessary to constrain the models in a quantitative manner. Rough estimates can always be made (section II-C). Some qualitative observational constraints are also taken into account. In the model solutions presented in chapter $\mathrm{V}$, for instance, it is required that the ocean be in motion everywhere. Indeed, if a region of the ocean was motionless for more than a few centuries, the ${ }^{14} \mathrm{C}$ age of the water in this region would become much larger than what can be reasonably accepted in view of the available data. This constraint that the ocean be in motion everywhere is qualitative in the sense that no lower bound on the rate of ventilation is imposed strictly, but in general, circulations which are too sluggish will not be accepted as reasonable solutions. 
The role of the equation imbalance term is to ensure that model results are consistent with the physics of the ocean. The oceanic circulation predicted by the model should conserve mass and the various geochemical tracers present in the models. It should also be in geostrophic balance. The model equations are not the true representation of the physics of the ocean because of the numerical approximations made, or because a treatment of the complete physical equations is not possible. The exception is the equation of mass conservation written in flux form. This equation should be exactly satisfied by the solution.

The formulation of the objective function is crucial for the success of the optimization. The objective function must be complete enough to ensure that the solution satisfies reasonably well all the equations of the model. The satisfaction of other criteria may also be desirable. In the modern ocean simulation described in chapter $\mathrm{V}$ for instance, it is required that the estimated circulation be relatively swift. This result is achieved by adding a term to the objective function, which constrains the density gradients to remain steep. At the same time, the objective function must be simple enough so that its minimization can be done in practice. Quadratic cost functions are the easiest to minimize because no iterative procedure is required. More generally, the smoothness of the objective function and of its derivatives is appreciable (Gill et al., 1981).

Several objective functions have been tried in this work, and are summarized below. They usually consist of simple polynomial functions of the model variables. Because these objective functions and their derivatives are continuous, they can be minimized using relatively simple optimization procedures. These functions are of higher order than simple quadratic functions because the model equation imbalances are nonlinear. The optimization, however, is done by iteratively linearizing these imbalances until convergence is obtained, and as explained in sections 1 and 2, the objective function is approximated by a quadratic function at each iteration of the minimization. The minimax objective function is described in section 3 . It is relatively more difficult to minimize because it is not continuous. Its minimization is done using a routine of the MATLAB Optimization Toolbox. Section 4 discusses the problems associated with local minima of objective functions. 


\section{1) Least square objective function}

The least square objective function used in the model of chapter IV is similar to that used by Mercier (1986) in a non linear inverse model of the oceanic circulation. The idea is to find a best fit between the observations and the model equations in a least square sense. The mathematical expression of the objective function is:

$$
\mathrm{J}=\left(\mathbf{x}-\mathbf{x}_{0}\right)^{\mathrm{T}} \cdot \mathbf{C}_{0}^{-1} \cdot\left(\mathbf{x}-\mathbf{x}_{0}\right)+\mathbf{f}(\mathbf{x})^{\mathrm{T}} \cdot \mathbf{C}_{\mathrm{f}^{-1}} \cdot \mathbf{f}(\mathbf{x})
$$

where $\mathbf{x}$ is the state vector. It is defined as the vector containing those elements of the model employed to describe the ocean: tracer concentrations and volume transports at each grid-point of the model. For the model described in chapter IV, there are about 800 such elements of the state vector.

$\mathbf{x}_{0}$ is the a priori estimate of the state vector. For the elements of the state vector which are constrained by observations, the $a$ priori estimate is the observation itself ${ }^{6}$. For the other elements, the a priori estimate could be a reasonable guess (obtained by extrapolation or interpolation for instance).

$\mathrm{C}_{0}$ is the covariance matrix of the a priori estimate of the solution. Its diagonal terms corresponding to elements of the state vector constrained by observations represent the error variance of these observations. Diagonal terms corresponding to elements of the state vector notconstrained by observations represent the uncertainty of the reasonable guess of the a priori solution. The latter are usually large so that the guess does not overly constrain the solution. The non diagonal terms of $\mathbf{C}_{0}$ represent the correlation between the uncertainties of the various elements of the state vector. In the model introduced in the next chapter, it is assumed that the uncertainties in the a priori estimate of the state vector are decorrelated $\left(\mathbf{C}_{0}\right.$ diagonal, see section IV-4).

6 Note that a more general formulation of the objective function would take into account the fact that the observations do not always directly constrain elements of the state vector. Often, they constrain a linear combination of the elements of $\mathbf{x}: \mathbf{y}=\mathbf{E x}+\mathbf{n}$, where $\mathbf{y}$ represents the observations, $\mathbf{E}$ is a matrix, and $\mathbf{n}$ is the observational noise. In this case, one replaces the $\left(\mathbf{x}-\mathbf{x}_{0}\right)^{\mathrm{T}} \cdot \mathbf{C}_{0}{ }^{-1} \cdot\left(\mathbf{x}-\mathbf{x}_{0}\right)$ term in the objective function by (Ex$y)^{T} \cdot C_{E}-1 \cdot(E x-y)$, where $C_{E}$ is the covariance matrix of the observational noise. The formulation of the objective function given above is appropriate for the problems treated in this thesis, however, because the observations of tracer distributions are assumed to directly constrain the tracer concentrations at model grid-points (see section IV-4). 
$\mathbf{f}(\mathbf{x})$ is a vector which contains the model equation imbalances, like the mass imbalance in each box for instance. If the model equations are exactly satisfied, $\mathbf{f}(\mathbf{x})=\mathbf{0}$ (imbalances $=0$ ). The elements of $\mathbf{f}$ which represent the tracer conservation equations are nonlinear because they are the product of a mass transport term by a tracer concentration term, both terms being variable in the model.

$\mathrm{C}_{\mathrm{f}}$ represents the covariance matrix of the uncertainty in the model equations. Small values of $\mathbf{C}_{\mathrm{f}}$ mean that the equations are a reliable representation of the physics of the ocean, and that they should be almost exactly satisfied. Note that if $\mathbf{C}_{\mathrm{f}}$ is small, $\mathbf{C}_{\mathrm{f}}{ }^{-1}$ is large, which means that large weighing coefficients are put on the physical constraints in the objective function.

There are various algorithms to minimize this least square objective function. The simplest one is the so-called Gauss-Newton method (Gill et al., 1981). The idea is to replace the physical constraints by a linearized approximation. First, one linearizes $\mathbf{f}(\mathbf{x})$ around a starting point $\mathbf{x}_{1}$ :

$$
\text { (2) } \mathbf{f}(\mathbf{x}) \approx \mathbf{f}\left(\mathbf{x}_{1}\right)+\mathbf{F}_{1}\left(\mathbf{x}-\mathbf{x}_{1}\right)
$$

where $\mathbf{F}_{1}$ is the jacobian matrix (matrix of first partial derivatives) of $\mathbf{f}$ estimated at $\mathbf{x}=\mathbf{x}_{1}$. The starting point, $\mathbf{x}_{1}$, can be the $a$ priori estimate of the solution, $\mathbf{x}_{0}$. Then, one minimizes an approximated objective function, $\mathrm{J}_{1}$, obtained by plugging the linearized expression of $\mathbf{f}$ in the true objective function, $\mathrm{J}$ :

$$
\mathbf{J}_{1}=\left(\mathbf{x}-\mathbf{x}_{0}\right)^{\mathrm{T}} \cdot \mathbf{C}_{0}^{-1} \cdot\left(\mathbf{x}-\mathbf{x}_{0}\right)+\left(\mathbf{F}_{1}\left(\mathbf{x}-\mathbf{x}_{1}\right)+\mathbf{f}\left(\mathbf{x}_{1}\right)\right)^{\mathrm{T}} \cdot \mathbf{C}_{\mathbf{f}}^{-1} \cdot\left(\mathbf{F}_{1}\left(\mathbf{x}-\mathbf{x}_{1}\right)+\mathbf{f}\left(\mathbf{x}_{1}\right)\right)
$$

One obtains a new estimate of the state vector, $\mathbf{x}_{2}$. The minimization of $\mathrm{J}_{1}$ is straightforward because $\mathrm{J}_{1}$ is quadratic. The operation is repeated replacing $\mathbf{x}_{1}$ by $\mathbf{x}_{2}$, and so on until convergence. The Gauss-Newton method usually converges toward a local minimum of the true objective function. If the procedure does not converge, it is necessary to try different starting points, or to modify some of the a priori guesses of variables which are not constrained by any observations (see section IV-6). The procedure is considered to have converged if there is little progress made at each step:

$$
\text { (4) } \frac{\left\|\mathbf{x}_{\mathrm{k}+1}-\mathbf{x}_{\mathrm{k}}\right\|}{\left\|\mathbf{x}_{\mathrm{k}}\right\|} \leq \varepsilon
$$

where $\mathbf{x}_{\mathrm{k}}$ and $\mathbf{x}_{\mathrm{k}+1}$ are the estimates of the state vector at iteration $\mathrm{k}$ and $\mathrm{k}+1$. The tolerance $\varepsilon$ is a small number. At convergence, $\mathbf{f}(\mathbf{x})$ is not exactly equal to zero, but is small. Thus, the model equations are not exactly satisfied, but the equation imbalances are small. As mentioned above, 
finding a solution which satisfies the equations exactly is not desirable because the equations are not an exact representation of the physics.

The progression from iteration $\mathrm{k}$ to iteration $\mathrm{k}+1$ is as follows:

find $\mathbf{x}_{\mathrm{k}+1}$ which minimizes:

$$
\mathbf{J}_{\mathrm{k}}=\left(\mathbf{x}-\mathbf{x}_{0}\right)^{\mathrm{T}} \cdot \mathbf{C}_{0}^{-1} \cdot\left(\mathbf{x}-\mathbf{x}_{0}\right)+\left(\mathbf{F}_{\mathrm{k}}\left(\mathbf{x}-\mathbf{x}_{\mathrm{k}}\right)+\mathbf{f}\left(\mathbf{x}_{\mathrm{k}}\right)\right)^{\mathrm{T}} \cdot \mathbf{C}_{\mathrm{f}}{ }^{-1} \cdot\left(\mathbf{F}_{\mathrm{k}}\left(\mathbf{x}-\mathbf{x}_{\mathrm{k}}\right)+\mathbf{f}\left(\mathbf{x}_{\mathrm{k}}\right)\right)
$$

$\mathbf{x}_{\mathrm{k}+1}$ satisfies:

$$
\begin{gathered}
\frac{\partial \mathrm{J}_{\mathrm{k}}}{\partial \mathbf{x}}=0 \\
==(6) \quad\left(\mathbf{C}_{0}^{-1}+\mathbf{F}_{\mathrm{k}}^{\mathrm{T}} \cdot \mathbf{C}_{\mathrm{f}}^{-1} \cdot \mathbf{F}_{\mathrm{k}}\right) \mathbf{x}_{\mathrm{k}+1}=\mathbf{C}_{0}^{-1} \cdot \mathbf{x}_{0}+\mathbf{F}_{\mathrm{k}}^{\mathrm{T}} \cdot \mathbf{C}_{\mathrm{f}}^{-1} \cdot\left(\mathbf{F}_{\mathrm{k}} \cdot \mathbf{x}_{\mathrm{k}}-\mathbf{f}\left(\mathbf{x}_{\mathrm{k}}\right)\right)
\end{gathered}
$$

Using the matrix inversion lemma (Liebelt, 1967), and after some algebraic manipulations, one obtains:

$$
\mathbf{x}_{\mathrm{k}+1}=\mathbf{x}_{0}+\mathbf{C}_{0} \cdot \mathbf{F}_{\mathrm{k}}{ }^{\mathrm{T}} \cdot\left[\mathbf{F}_{\mathrm{k}} \cdot \mathbf{C}_{0} \cdot \mathbf{F}_{\mathrm{k}}^{\mathrm{T}}+\mathbf{C}_{\mathrm{f}}\right]^{-1} \cdot\left[\mathbf{F}_{\mathrm{k}} \cdot\left(\mathbf{x}_{\mathrm{k}}-\mathbf{x}_{0}\right)-\mathbf{f}\left(\mathbf{x}_{\mathrm{k}}\right)\right]
$$

Note that the Hessian matrix (matrix of second partial derivatives) of $\mathbf{f}(\mathbf{x})^{\mathrm{T}} \cdot \mathbf{C}_{\mathrm{f}}{ }^{-1} \cdot \mathbf{f}(\mathbf{x})$ is approximated at each iteration by a term proportional to the square of the jacobian matrix of $\mathbf{f}(\mathbf{x})$ estimated at $\mathbf{x}=\mathbf{x}_{\mathrm{k}}, \mathbf{F}_{\mathrm{k}}^{\mathrm{T}} \cdot \mathbf{C}_{\mathrm{f}}{ }^{-1} \cdot \mathbf{F}_{\mathrm{k}}$. This approximation is characteristic of the Gauss-Newton method (Gill et al., 1981). It is justified when one expects $\mathbf{f}(\mathbf{x})$ to be small for $\mathbf{x}$ close to the minimum of the objective function (Gill et al., 1981). In the model described in chapter IV, $\mathbf{f}(\mathbf{x})$ represents the model equation imbalances. These imbalances must be small for the solution to be acceptable, and thus the Gauss-Newton method is appropriate for this model.

Note that if the model uncertainty is zero, $\mathbf{C}_{\mathrm{f}}=\mathbf{0}$, one retrieves the expression derived by Tarantola and Valette $(1982 b)^{7}$ for problems where the physical constraints should be satisfied

7 The problem examined by Tarantola and Valette (1982b) is to find a solution $\mathbf{x}$ which minimizes the data misfit, while exactly satisfying the model equations:

(8) minimize $J=\left(\mathbf{x}-\mathbf{x}_{0}\right)^{\mathrm{T}} \cdot \mathbf{C}_{0}-1 \cdot\left(\mathbf{x}-\mathbf{x}_{0}\right)$ subject to $\mathbf{f}(\mathbf{x})=\mathbf{0}$

They solve this problem using a geometrical argument and a fixed point method. Another method to solve (8) is to rewrite the problem using Lagrange multipliers. The solution of:

(9) minimize $\mathrm{J}^{\prime}=\left(\mathbf{x}-\mathbf{x}_{0}\right)^{\mathrm{T}} \cdot \mathbf{C}_{0}^{-1} \cdot\left(\mathbf{x}-\mathbf{x}_{0}\right)-2 \mu^{\mathrm{T}} \cdot \mathbf{f}(\mathbf{x})$

where $\mu$ represents the Lagrange multipliers, is also solution of problem (8). Indeed, the solution of (9) satisfies: $\partial J^{\prime} / \partial \mu=0$, which implies $\mathbf{f}(\mathbf{x})=\mathbf{0}$, so both the solutions of problem (8) and problem (9) belong to the manifold defined by $\mathbf{f}(\mathbf{x})=\mathbf{0}$; since $\mathbf{J}=\mathrm{J}^{\prime}$ in this manifold, they have the same minimum. One way to solve problem (9) is to iteratively linearize $\mathbf{f}(\mathbf{x})$ until convergence is achieved:
(10)
$\mathbf{f}(\mathbf{x})=\mathbf{0} \approx \mathbf{f}\left(\mathbf{x}_{\mathrm{k}}\right)+\mathrm{F}_{\mathrm{k}}\left(\mathbf{x}-\mathbf{x}_{\mathrm{k}}\right)$
$\Longrightarrow(11)$
$\mathbf{F}_{\mathrm{k}} \mathbf{x}_{\mathrm{k}+1}=\mathbf{F}_{\mathrm{k}} \mathbf{x}_{\mathrm{k}}-\mathbf{f}\left(\mathbf{x}_{\mathrm{k}}\right)$

$\mathbf{x}$ must also satisfy:

(12) $\quad \partial \mathrm{J}^{\prime} / \partial \mathrm{x}=\mathbf{0} \Rightarrow \mathrm{C}_{0}^{-1} \cdot\left(\mathbf{x}-\mathbf{x}_{0}\right)=\mathrm{F}_{\mathrm{k}}^{\mathrm{T}} \mu \quad \Rightarrow(13) \quad \mathbf{x}_{\mathrm{k}+1}=\mathbf{x}_{0}+\mathbf{C}_{0} \cdot \mathbf{F}_{\mathrm{k}}{ }^{\mathrm{T}} \cdot \mu$

plugging expression (13) in expression (11), one can estimate $\mu$, and using (13) again, calculate $\mathbf{x}_{\mathrm{k}+1}$ :

(14) $\quad \mathbf{x}_{\mathrm{k}+1}=\mathbf{x}_{0}+\mathbf{C}_{0} \cdot \mathbf{F}_{k^{T}} \cdot\left[\mathbf{F}_{\mathrm{k}} \cdot \mathbf{C}_{0} \cdot \mathbf{F}_{\mathrm{k}^{\mathrm{T}}}\right]^{-1} \cdot\left[\mathbf{F}_{\mathrm{k}} \cdot\left(\mathbf{x}_{\mathrm{k}}-\mathbf{x}_{0}\right)-\mathbf{f}\left(\mathbf{x}_{\mathrm{k}}\right)\right]$ 
exactly. The fact that (7) reduces to the expression derived by Tarantola and Valette in the context of model equations exactly satisfied is not surprising because $\mathbf{C}_{\mathrm{f}}=\mathbf{0}$ implies that $\mathbf{f}(\mathbf{x})=\mathbf{0}$ exactly, which corresponds to the problem they solved.

The approximated objective function, $\mathrm{J}_{\mathrm{k}}$, given by expression (5), is identical to a Recursive Least Square objective function (Wunsch, 1990). Thus the covariance matrix of the estimate $\mathbf{x}_{\mathrm{k}}$ can be updated at each iteration using the expression developed in the context of Recursive Least Square problems. Its expression at step $\mathrm{k}+1$ is:

$$
\text { (15) } \quad \mathbf{P}_{\mathrm{k}+1}=\left[\mathbf{I}-\mathbf{C}_{0} \cdot \mathbf{F}_{\mathrm{k}}^{\mathrm{T}} \cdot\left(\mathbf{F}_{\mathrm{k}} \cdot \mathbf{C}_{0} \cdot \mathbf{F}_{\mathrm{k}}^{\mathrm{T}}+\mathbf{C}_{\mathrm{f}}\right)^{-1} \cdot \mathbf{F}_{\mathrm{k}}\right] \mathbf{C}_{0}
$$

where I designates the identity matrix, and $\mathbf{P}_{\mathrm{k}+1}$ the covariance matrix of $\mathbf{x}_{\mathrm{k}+1}$. Note that it is not necessary to know the covariance matrix at each iteration (which requires intensive calculations) to compute $\mathbf{x}_{\mathrm{k}}$. One can estimate the covariance matrix of the final solution once convergence has been achieved. The diagonal terms of this matrix $\mathbf{P}_{\mathrm{k}}$ indicate the formal uncertainty of the estimated variables.

\section{2) A practical approach}

One difficulty with the algorithm presented above is that it involves the explicit inversion the Hessian matrix, $\mathrm{C}_{0}{ }^{-1}+\mathbf{F}_{\mathrm{k}}{ }^{\mathrm{T}} \cdot \mathbf{C}_{\mathrm{f}}{ }^{-1} \cdot \mathbf{F}_{\mathrm{k}}$, at each iteration. A modified formulation of the algorithm that avoids the inversion of this matrix is presented here.

Instead of using relation (7), $\mathbf{x}_{\mathrm{k}+1}$ is obtained by solving a linear system of equations, using efficient methods such as the $\mathrm{QR}$ decomposition. The size of this linear system is kept relatively small by separating the linear physical constraints from the nonlinear ones in the objective function:

$$
J=\left(\mathbf{x}-\mathbf{x}_{0}\right)^{\mathrm{T}} \mathbf{C}_{0}^{-1}\left(\mathbf{x}-\mathbf{x}_{0}\right)+(\mathbf{A x}-\mathbf{b})^{\mathrm{T}} \mathbf{C}_{A^{-1}}(\mathbf{A x}-\mathbf{b})+\mathbf{f}(\mathbf{x})^{\mathrm{T}} \cdot \mathbf{C}_{\mathbf{f}^{-1}} \cdot \mathbf{f}(\mathbf{x})
$$

where $\mathbf{A x}-\mathbf{b}$ represents the linear equation imbalances, and $\mathbf{f}(\mathbf{x})$ the nonlinear equation imbalances. Note that from now on, $\mathbf{f}(\mathbf{x})$ represents either the nonlinear equation imbalances

This expression, which is identical to the one derived by Tarantola and Valette (1982b), permits to go from step $\mathrm{k}$ to step $\mathrm{k}+1$ of the minimization. 
exclusively, or the equation imbalances in general. The meaning should be clear from the context. $\mathrm{C}_{\mathrm{A}}$ is the covariance matrix of the linear equation imbalances.

As in the previous sections, $\mathbf{f}(\mathbf{x})$ is replaced by a linearized expression at each iteration until convergence. The objective function is at a minimum if:

$$
\begin{gathered}
\frac{\partial \mathrm{J}}{\partial \mathbf{x}}=0 \\
=\Rightarrow(18) \quad\left(\mathbf{C}_{0}{ }^{-1}+\mathbf{A}^{\mathrm{T}} \cdot \mathbf{C}_{\mathrm{A}^{-1}} \cdot \mathbf{A}+\mathbf{F}_{\mathrm{k}}{ }^{\mathrm{T}} \cdot \mathbf{C}_{\mathrm{f}}{ }^{-1} \cdot \mathbf{F}_{\mathrm{k}}\right) \mathbf{x}_{\mathrm{k}+1}= \\
\mathbf{C}_{0}-1 \cdot \mathbf{x}_{0}+\mathbf{A}^{\mathrm{T}} \mathbf{C}_{\mathrm{A}^{-1}}\left(\mathbf{A} \cdot \mathbf{x}_{\mathrm{k}}-\mathbf{b}\right)+\mathbf{F}_{\mathrm{k}}^{\mathrm{T}} \cdot \mathbf{C}_{\mathrm{f}}{ }^{-1} \cdot\left(\mathbf{F}_{\mathrm{k}} \cdot \mathbf{x}_{\mathrm{k}}-\mathbf{f}\left(\mathbf{x}_{\mathrm{k}}\right)\right)
\end{gathered}
$$

Defining $\mathrm{C}_{0 \mathrm{~A}}$ to be such that:

$$
\mathbf{C}_{0 A^{-1}}=\mathbf{C}_{0}-1+\mathbf{A}^{\mathrm{T}} \cdot \mathbf{C}_{\mathrm{A}^{-1}} \cdot \mathbf{A}
$$

and using the matrix inversion lemma, one obtains:

$$
\begin{aligned}
& \left(\mathbf{C}_{0}{ }^{-1}+\mathbf{A}^{\mathrm{T}} \cdot \mathbf{C}_{\mathrm{A}}^{-1} \cdot \mathbf{A}+\mathbf{F}_{\mathrm{k}}^{\mathrm{T}} \cdot \mathbf{C}_{\mathrm{f}}^{-1} \cdot \mathbf{F}_{\mathrm{k}}\right)^{-1}=\left(\mathbf{C}_{0 A^{-1}}+\mathbf{F}_{\mathrm{k}}^{\mathrm{T}} \cdot \mathbf{C}_{\mathrm{f}}{ }^{-1} \cdot \mathbf{F}_{\mathrm{k}}\right)^{-1} \\
& =\mathrm{C}_{0 \mathrm{~A}}-\mathrm{C}_{0 \mathrm{~A}} \cdot \mathbf{F}_{\mathrm{k}}^{\mathrm{T}}\left(\mathbf{F}_{\mathrm{k}} \cdot \mathrm{C}_{0 \mathrm{~A}} \cdot \mathbf{F}_{\mathrm{k}}^{\mathrm{T}}+\mathbf{C}_{\mathrm{f}}\right)^{-1} \cdot \mathbf{F}_{\mathrm{k}} \cdot \mathbf{C}_{0 \mathrm{~A}}
\end{aligned}
$$

Introducing for the right hand side of (18):

$$
\mathbf{b}=\mathbf{C}_{0}^{-1} \cdot \mathbf{x}_{0}+\mathbf{A}^{\mathrm{T}} \mathbf{C}_{\mathrm{A}^{-1}}\left(\mathbf{A} \cdot \mathbf{x}_{\mathrm{k}}-\mathbf{b}\right)+\mathbf{F}_{\mathrm{k}}^{\mathrm{T}} \cdot \mathbf{C}_{\mathrm{f}}^{-1} \cdot\left(\mathbf{F}_{\mathrm{k}} \cdot \mathbf{x}_{\mathrm{k}}-\mathbf{f}\left(\mathbf{x}_{\mathrm{k}}\right)\right)
$$

(b is a known vector) and:

$$
\alpha=\left(\mathbf{F}_{\mathrm{k}} \cdot \mathbf{C}_{0 \mathrm{~A}} \cdot \mathbf{F}_{\mathrm{k}}^{\mathrm{T}}+\mathbf{C}_{\mathrm{f}}\right)^{-1} \cdot \mathbf{F}_{\mathrm{k}} \cdot \mathbf{C}_{0 \mathrm{~A}} \cdot \mathbf{b}
$$

one can express $\mathbf{x}_{\mathrm{k}+1}$ in function of $\mathbf{b}$ and $\alpha$ :

$$
\mathbf{x}_{\mathrm{k}+1}=\left(\mathbf{C}_{0 \mathrm{~A}}{ }^{-1}+\mathbf{F}_{\mathrm{k}}^{\mathrm{T}} \cdot \mathbf{C}_{\mathrm{f}}^{-1} \cdot \mathbf{F}_{\mathrm{k}}\right)^{-1} \cdot \mathbf{b}=\mathbf{C}_{0 \mathrm{~A}} \cdot \mathbf{b}-\mathbf{C}_{0 \mathrm{~A}} \cdot \mathbf{F}_{\mathrm{k}}^{\mathrm{T}} \cdot \alpha
$$

$\alpha$ can be computed by solving the linear system (without matrix inversion):

$$
\left(\mathbf{F}_{\mathrm{k}} \cdot \mathrm{C}_{0 \mathrm{~A}} \cdot \mathbf{F}_{\mathrm{k}}^{\mathrm{T}}+\mathrm{C}_{\mathrm{f}}\right) \cdot \alpha=\mathbf{F}_{\mathrm{k}} \cdot \mathbf{C}_{0 \mathrm{~A}} \cdot \mathbf{b}
$$

This linear system has the dimension of $\mathrm{C}_{\mathrm{f}}, \mathrm{m} \times \mathrm{m}$, where $\mathrm{m}$ is the number of nonlinear physical constraints $^{8}$. Solving this system takes most of the computing time, so it worth using an efficient procedure. In the model described in chapter IV, the MATLAB "I" operator is used. It relies upon a QR factorization of $\left(\mathbf{F}_{\mathrm{k}} \cdot \mathbf{C}_{0 \mathrm{~A}} \cdot \mathbf{F}_{\mathrm{k}}^{\mathrm{T}}+\mathbf{C}_{\mathrm{f}}\right)$. The other computationally intensive operation is the inversion: $\mathbf{C}_{0 \mathrm{~A}}=\left(\mathbf{C}_{0}^{-1}+\mathbf{A}^{\mathrm{T}} \cdot \mathbf{C}_{\mathrm{A}}{ }^{-1} \cdot \mathbf{A}\right)^{-1}$. It must be done only once though, at the beginning of the optimization procedure. $\mathbf{C}_{0 \mathrm{~A}}$, however, must be stored until convergence is achieved.

\footnotetext{
8 If one does not separate linear from nonlinear constraints, one must solve a system of dimension m' $\times \mathrm{m}^{\prime}$ where $\mathrm{m}$ ' is the total number of physical constraints (linear + nonlinear).
} 
Note that the covariance matrices $\mathrm{C}_{0}, \mathrm{C}_{\mathrm{A}}$, and $\mathrm{C}_{\mathrm{f}}$ are diagonal matrices, and the use of a sparse matrix algorithm can greatly reduce the computational load. For the model presented in the following chapter (about 800 variables and 1000 equations, about 300 being nonlinear), using the MATLAB sparse matrix algorithm reduces the calculation time from several hours down to 10 minutes on a Sparc 2 workstation.

\section{3) Minimax algorithm (for small systems only)}

A simple kinematic 5 box-model of the South Atlantic is introduced in chapter V. The model is constrained to produce tracer concentrations consistent with South Atlantic data, using an optimization method called minimax. The idea behind the minimax method is to find a state vector $\mathbf{x}$ which minimizes the maximum equation imbalance (Gill et al., 1981). $\mathbf{x}$ is constrained to be within an interval which represents the range of plausible values that the model variables can take. The optimization problem is:

$$
\begin{gathered}
\min \mathrm{J}=\min \left(\max \left|\mathrm{f}_{\mathrm{i}}(\mathbf{x})\right|\right) \\
\mathrm{i} \in \mathrm{I} \\
\mathbf{v}_{\mathrm{lb}}<\mathbf{x}<\mathbf{v}_{\mathrm{ub}}
\end{gathered}
$$

where $f_{i}$ represents equation number $i$ of the model, I represents the set of equations, and $\mathbf{v}_{\mathrm{lb}}$ and $\mathbf{v}_{\mathrm{ub}}$ are the lower and upper bounds within which the state vector must be. For model variables constrained by observations, $\mathbf{v}_{\mathrm{lb}}$ and $\mathbf{v}_{\mathrm{ub}}$ represent bounds consistent with the observational uncertainties ( \pm 2 standard deviations away from the observed value, for instance). For model variables not constrained by observations, $\mathbf{v}_{\mathrm{lb}}$ and $\mathbf{v}_{\mathrm{ub}}$ are reasonable bounds, chosen to be large enough so to not artificially constrain the solution. $\mathbf{v}_{\mathrm{lb}}$ and $\mathbf{v}_{\mathrm{ub}}$ play a role similar to that of the data misfit term in the least square objective function, by constraining the solution to be consistent with the observations within the observational uncertainty.

The minimax method tends to produce solutions such that $\mathrm{f}_{\mathrm{i}}(\mathbf{x})$ has the same value for all $\mathrm{i}$ at the minimum of the objective function. This property is convenient because it guarantees that the imbalances are not concentrated in one box. With the Least Square methods described in the previous sections, often one obtains solutions such that a given tracer is conserved in every box, except one, where it is not conserved at all. This large imbalance concentrated in one box 
produces a large artificial source which can alter the solution. The minimax approach is efficient in the sense that it usually converges toward an acceptable solution, if such a solution exists. The drawback of this approach is that it is slow and cannot be used for problems that involve several hundreds of variables.

\section{4) Local minima}

There is no guarantee that most optimization procedures will find the global minimum of the cost function. Note that one solution is to use optimization methods like simulated annealing which can escape from local minima (Barth and Wunsch, 1990; Kruger, 1993). However, these methods tend to require large amounts of computation, so it is worth trying to go as far as possible with more classical methods.

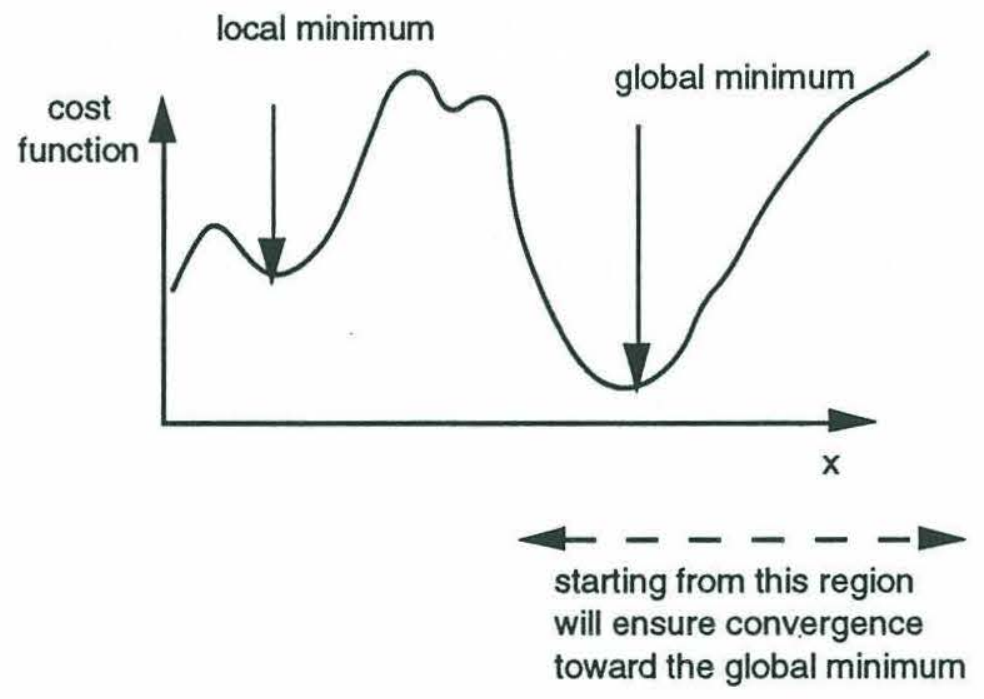

Figure III-1: illustrates the importance of the starting point

In theory, it should be possible to find the global minimum of the objective function by trying different starting points (Figure III-1). However, with a number of variables close to one thousand in the model described in chapter IV, it is impossible to try every combination. Starting from the a priori estimate of the solution usually gives the best results, but this approach is not 
always ideal either because many variables of the model are not constrained by any observations, and their a priori estimate is only a guess which can be far from the optimal value.

Local minima do not always result in unacceptable solutions. Multiple minima may correspond to multiple acceptable solutions indistinguishable within the observational error. In this case, it is not necessary to find the global minimum.

For the cost function of section 1, one way to understand the minimum cost solution is to consider it as the most likely solution. If we assume the error in the a priori estimate of the solution and the physical constraints to have a gaussian distribution and the weight matrices $\mathrm{C}_{0}$ and $\mathbf{C}_{\mathrm{f}}$ to be their covariance matrices, then their probability distributions are:

$$
\begin{aligned}
& \rho(\mathbf{x})=\alpha \exp \left\{-\frac{1}{2}\left(\mathbf{x}-\mathbf{x}_{0}\right)^{\mathrm{T}} \mathbf{C}_{0}^{-1}\left(\mathbf{x}-\mathbf{x}_{0}\right)\right\} \\
& \theta(\mathbf{x})=\beta \exp \left\{-\frac{1}{2} \mathbf{f}(\mathbf{x})^{\mathrm{T}} \mathbf{C}_{\mathrm{f}}^{-1} \mathbf{f}(\mathbf{x})\right\}
\end{aligned}
$$

where $\alpha$ and $\beta$ are normalization factors. The conjunction of $\rho(\mathbf{x})$ and $\Theta(\mathbf{x})$ gives a new state of information with a probability density function:

$$
\sigma(\mathbf{x})=\rho(\mathbf{x}) \cdot \theta(\mathbf{x})=\gamma \exp \left\{-\frac{1}{2}\left[\left(\mathbf{x}-\mathbf{x}_{0}\right)^{\mathrm{T}} \mathbf{C}_{0}^{-1}\left(\mathbf{x}-\mathbf{x}_{0}\right)+\mathrm{f}(\mathbf{x})^{\mathrm{T}} \mathbf{C}_{\mathrm{f}}^{-1} \mathbf{f}(\mathbf{x})\right]\right\}
$$

where $\gamma$ is a normalization factor. The vector $\underline{\mathbf{x}}$ which minimizes the objective function:

$$
J=\left(\mathbf{x}-\mathbf{x}_{0}\right)^{\mathrm{T}} \mathbf{C}_{0}^{-1}\left(\mathbf{x}-\mathbf{x}_{0}\right)+\mathrm{f}(\mathbf{x})^{\mathrm{T}} \mathbf{C}_{\mathrm{f}}^{-1} \mathbf{f}(\mathbf{x})
$$

maximizes the probability density function $\sigma$. Thus, $\underline{\mathbf{x}}$ is the position where the state vector is most likely to be, given the conjunction of the data and the model physics (see Tarantola and Valette,1982, for a more thorough discussion). If $\underline{\mathbf{x}}$ is only close to minimizing the cost function, it is not the most likely solution, but it still represents a probable state. In the model, $\mathbf{C}_{0}$ and $\mathrm{C}_{\mathrm{f}}$ are only approximations to the true covariance matrices, and thus even the state vector corresponding to the global minimum of the objective function is an approximation itself. 


\section{Chapter IV - A Coarse resolution inverse model of the North Atlantic.}

This chapter describes a non-linear inverse model which will be used to simulate the glacial North Atlantic. We employ a model similar to the one developed by Mercier (1989) to determine a solution that best fits physical and observational constraints through the minimization of a cost function. Primary innovations of this model include its use for paleoceanographic studies and its treatment of temperature and salinity as variables that are linked to density through a state equation. In other non-linear models (e.g., Mercier, 1989; Mercier et al., 1993), density can be adjusted but not temperature and salinity.

The following paragraphs describe the model domain and the numerical representation of the physics. We then examine the mathematical formalism used to combine the physics and the data. The last section describes some problems commonly encountered when using the model.

\section{1) Model domain}

We use a finite difference model of the North Atlantic which extends from $10^{\circ} \mathrm{N}$ to $50^{\circ} \mathrm{N}$, and from a depth of 1 kilometer to the bottom of the ocean (Figure IV-1). The boundary conditions at $10^{\circ} \mathrm{N}, 50^{\circ} \mathrm{N}$, and at a depth of $1 \mathrm{~km}$ are treated as variables obtained from the inversion. In other words, the model determines boundary conditions that are consistent with the interior data and physical constraints. The model domain is limited to the North Atlantic because the available data set lies predominantly in this region. The domain is sufficiently large to estimate general circulation changes. $50^{\circ} \mathrm{N}$ is very close to the regions of formation of NADW, and $10^{\circ} \mathrm{N}$ is sufficiently far south for the model to feel the influence of Antarctic Bottom Water. A $\delta^{13} \mathrm{C}$. meridional section in the modern ocean clearly shows Bottom Water very depleted in carbon 13 north of the equator. (Figure II-B-2; Kroopnick,1985). The last glacial maximum data are usually interpreted as a sign that Antarctic Bottom Water penetrated farther north during the Last Ice Age, and the largest changes in the water mass distribution seem to have taken place in the North 


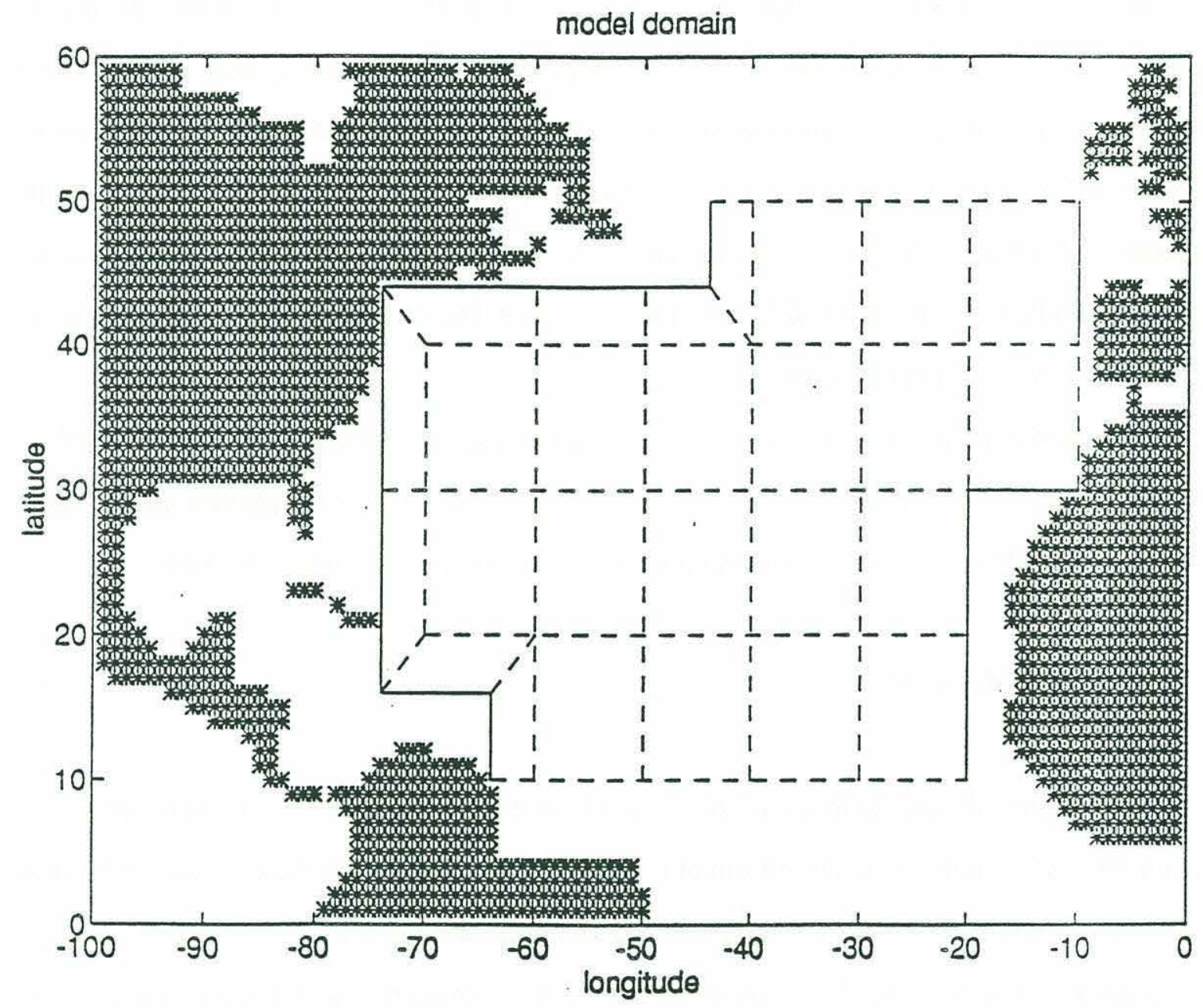

Figure IV-1: Horizontal grid of the model. Tracer concentrations are located at comers of volume elements. Volume transports are located at center of element interfices. The westernmost series of boxes represent the DWBC. 
Atlantic. In addition, limiting the study to the North Atlantic keeps the size of the problem tractable; there are about 850 variables and 1000 equations in the dynamical box-model (all the equations are not imposed exactly, so it is possible to have more equations than unknowns).

The thermocline and the mixed layer are not included in the model. There are not many data in the thermocline, and it is not clear whether $\delta^{13} \mathrm{C}$, the main source of information, is reliably recorded by planktonic foraminifera in the mixed layer (Keigwin and Boyle, 1989). Very few studies have used planktonic $\delta^{13} \mathrm{C}$ so far, one noticeable exception being the reconstruction of Duplessy et al. (1988). They contour isolines of $\delta^{13} \mathrm{C}$ in the Eastern Atlantic by directly linking planktonic and benthic foraminifera data points, but their reconstruction appears to be inconsistent with more recent data (see chapter II). It is unlikely that the surface data could be linked to deep ocean data without introducing fairly complicated patterns in the $\delta^{13} \mathrm{C}$ distribution in the thermocline. Doing so would require the addition of several layers in the model, and greatly increase the number of variables; as a result, explicit modeling of the upper ocean is postponed until more data become available in the thermocline.

There is a potential drawback of working in a limited domain. It is possible that some pieces of information could be missed because other ocean basins and the upper layers of the ocean are not included in the model. Worse, the results of the inversions could be inconsistent with data from outside the model domain. There is no guarantee, for instance, that the volume fluxes across the boundaries of the domain estimated by the North Atlantic model are consistent with data from the South Atlantic. In order to check the compatibility of the model results with data from other regions of the ocean, simulations of the North Atlantic circulation are systematically extended to the South Atlantic using a kinematic 5-box model. This kinematic box-model is described in the next chapter. It does not include any dynamical balances, but should be sufficient to tell if the circulation estimated in the North Atlantic model is grossly inconsistent with the South Atlantic data. 


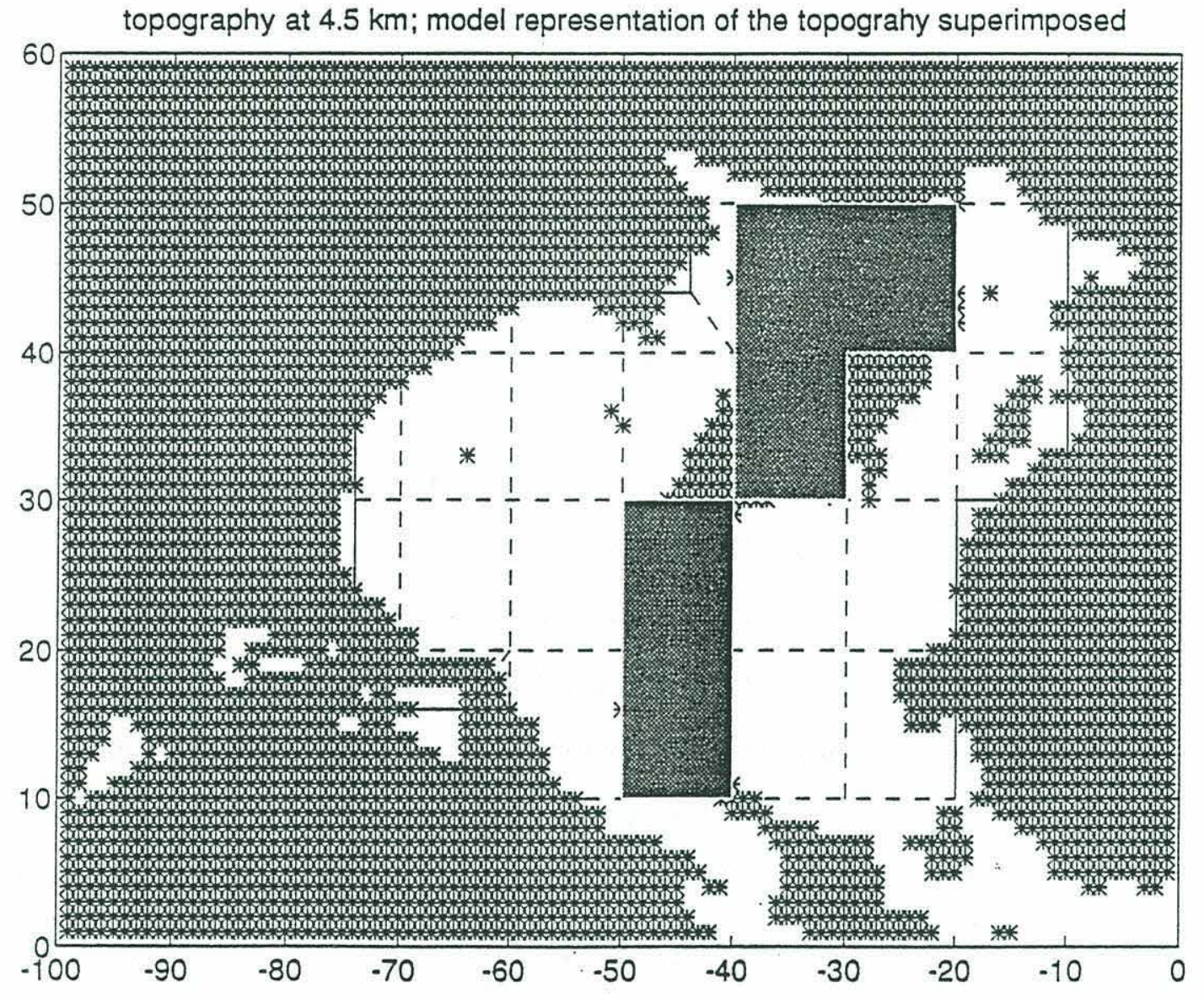

Figure IV-2: Topography in the North Atlantic at $4.5 \mathrm{~km}$ depth. Model grid superimposed. The Mid-Atlantic ridge is simulated by a series of "walls" (in black in the figure) through which there is no flow. The DWBC boxes are assumed to be open ocean boxes in the model, even if their geographical location corresponds to land areas in the real world. 


\section{2) Numerical representation of the physics}

The dynamical box-model includes a simplified representation of the physics of the ocean circulation: linear vorticity and thermal wind balances, conservation of mass, potential temperature, salinity and a third tracer (either phosphorus or ${ }^{13} \mathrm{C}$ ). These physical constraints are commonly used in inverse modeling. They are introduced into the model as finite difference equations. The model domain is divided into volumes of $10^{\circ} \times 10^{\circ} \times 1 \mathrm{~km}$, and conservation statements are written for each of these volumes. A series of 32 boxes is added to the western side of the model to simulate the deep western boundary current. These boxes are treated in the same way as the interior boxes except that the linear vorticity balance is not imposed since one expects higher order physics in the deep western boundary current (Stommel and Arons, 1960a\&b). There is no flow through the eastern and western boundaries, the Mid-Atlantic Ridge and the bottom of the ocean. The Mid-Atlantic Ridge is crudely represented at depths below 3 and $4 \mathrm{~km}$ (Figure IV-2). Thus, the deep Eastern Atlantic is isolated from its Western counterpart. The finite difference grid corresponds to a scheme D in Arakawa and Lamb's classification (Arakawa and lamb, 1977), with the vertical velocity w located at the center of the lower and upper faces of the cube.

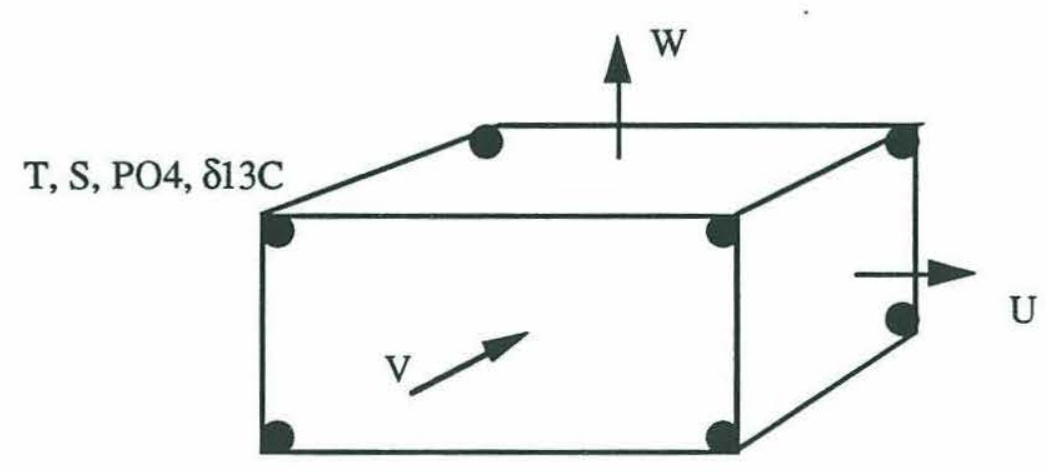

Figure IV-3: Grid arrangement. The tracer concentrations are defined at the corners of the box and the transports at the center of each side. The tracer concentration advected across a side is taken to be the mean of the 4 corner values. 
The main advantage of this grid is to allow for an easy discretization of the thermal wind and mass balances. Some averaging of the tracer field is necessary to compute tracer fluxes (the tracer concentration at the center of each side of the volume element is approximated by the mean of the four corner concentrations). The same numerical scheme is used by Mercier et al. (1993) in their non-linear inverse model.

We choose a very coarse resolution because of the incomplete geographical coverage of the data set (see map of data distribution, Figure II-C-1). Even on such a coarse resolution grid, there are many grid points which are not constrained by any observations, especially in the Western Atlantic. As more data become available, some of the gaps will be filled, but there are areas in the ocean which will not be covered such as shallow waters away from the coast line, or below the calcium compensation depth where foraminifera shells dissolve. There are no reasons why one could not assimilate scarce data in a high resolution model, but a low resolution model, which can be run very quickly (about 10 minutes on Sun Sparc 2 workstation), is more practical at present. Moreover, we will see in the next chapter that the paleo-data provide only weak constraints on the model circulation. This conclusion would not be altered by using a higher resolution model since more degrees of freedom to fit the same limited number of data points would further weaken the constraints. Furthermore, part V-A shows that the model is consistent with modern ocean data and produces a reasonable solution. The greater density of boxes near the western boundary is beneficial as it provides the model with a higher resolution where the circulation is most energetic.

\section{3) Physical constraints}

This section describes how the physical constraints are implemented in the model.

\section{a/ Mass conservation}

Mass conservation can be written in a flux form for any volume without numerical approximation. In the model, mass conservation is imposed in each box by minimizing $J_{M}$, the local mass imbalance contribution to the objective function: 


$$
J_{M}=\left(\sum_{i} U_{i}\right)^{2}
$$

where $U_{i}$ designates the volume fluxes going into the box, expressed in Sverdrups $\left(\mathrm{Sv}, 1 \mathrm{~Sv}=10^{6}\right.$ $\mathrm{m}^{3} / \mathrm{s}$ ). In practice, $J_{M}$ is multiplied by a weighting coefficient to control the minimization of the mass imbalance. Because the temperature and salinity fields can be adjusted in the model and we look for a steady state solution, there are no reasons why mass should not be exactly conserved. Moreover, if mass is not strictly conserved, tracer equations will depend on the units used to express the tracer concentrations (see following section) which is not physical. Thus we put a large weight on $J_{M}$ so that: $\Sigma_{\mathrm{i}} \mathrm{U}_{\mathrm{i}}=0$ almost perfectly in every box.

\section{b/ Potential temperature, salinity, phosphate and ${ }^{13} \mathrm{C}$ conservation}

Both salinity and potential temperature are conservative tracers. Phosphate is also conservative in the deep ocean in the absence of biological activity. In the modern North Atlantic, the low oxygen utilization rate suggests that deep waters are flushed rapidly, and the input of phosphorus

from the biological particle flux is small compared to the advective transports (Rintoul and Wunsch, 1991). Thus, neglecting the effect of biological activity in the Atlantic appears reasonable as a first approximation.

The sum of the fluxes of a conservative tracer into a volume should balance in the same way as it does for mass:

$$
\sum_{i} F_{C_{i}}=0
$$

$F_{C i}$, the flux of tracer $\mathrm{C}$ across side $\mathrm{S}_{\mathrm{i}}$ of a box, can be written as:

$$
F_{C_{i}}=\int_{S_{i}} u C d S
$$

where $\mathbf{u}$ is the velocity vector at side $S_{\mathrm{i}}$. In the model, $F_{C i}$ is approximated by $U_{i} \times C_{i}$ where:

$$
U_{i}=\int_{S_{i}} u d S
$$


is the integrated mass flux going into the box across $\mathrm{S}_{\mathrm{i}}$ and $\mathrm{C}_{\mathrm{i}}$ is an average concentration over side $S_{i}$ of the box (taken in the model as the mean of the 4 concentrations associated with the 4 corners of each side of the box, see Figure IV-3). Thus, the numerical approximation of tracer conservation equations is:

$$
\sum_{\mathrm{i}} U_{i} \times C_{i} \approx 0
$$

This numerical equation is taken into account in the model by minimizing the tracer imbalance contribution to the objective function:

$$
J c=\left(\sum_{i} U i \times C i\right)^{2}
$$

Because the numerical tracer conservation equation is only an approximation, it needs not be satisfied exactly by the model solution, and a relatively low weight is put on the minimization of $J c$. Note that if mass is not exactly conserved, tracer equations will depend on the units of the tracer concentration; writing the concentration as the sum of a mean term and a small departure from the mean:

$$
C_{i}=\bar{C}+\delta C_{i}
$$

and plugging this expression into the tracer equation, one obtains:

$$
\begin{gathered}
\sum_{i} U_{i} \times C_{i}=0=\bar{C} \times \sum_{i} U_{i}+\sum_{i} U_{i} \times \delta_{C_{i}} \\
\quad \neq 0
\end{gathered}
$$

Thus, if the tracer is salt for instance, expressing salinity using the Practical Salinity Scale would introduce a spurious source term:

$$
\bar{C} \times \Sigma_{i} U_{i}
$$

and the imbalance in the salinity conservation equation would depend on whether salinity is expressed using PSS or "PSS -35".

Different tracers are used for modern and LGM ocean simulations. 
1) modern ocean

Potential temperature, salinity, and phosphate are used as constraints on the oceanic circulation. We neglect the impact of particle fluxes so that phosphate is treated as a conservative tracer in the same way as potential temperature and salinity. As mentioned before, this assumption is reasonable in the Atlantic, and we will see in the next chapter that it is consistent with modern ocean data.

\section{2) LGM ocean}

The LGM circulation is constrained in the model by $\delta^{18} \mathrm{O}$ and $\delta^{13} \mathrm{C}$ data. $\delta^{18} \mathrm{O}$ data are converted to temperature variables according to the following relation:

$$
\mathrm{T}=-4.0\left(\delta^{18} \mathrm{O}_{\mathrm{C}}-\delta^{18} \mathrm{O}_{\mathrm{W}}\right)+16.9 \quad\left(\mathrm{~T} \text { in Celcius, } \delta^{18} \mathrm{O}\right. \text { in \%o; Shackleton, 1974) }
$$

where $\delta^{18} \mathrm{O}_{\mathrm{c}}$ is the $\delta^{18} \mathrm{O}$ value measured in foraminifera shells and $\delta^{18} \mathrm{O}_{\mathrm{W}}$ is the $\delta^{18} \mathrm{O}$ of sea water (unknown; see chapter II). Furthermore, potential temperature is assumed to be a function of temperature (T) and pressure (p) only:

$$
\Theta=\mathrm{T}+\mathrm{C}(\mathrm{p})
$$

Then: $\quad \Theta=-4.0\left(\delta^{18} \mathrm{O}_{\mathrm{c}}-\delta^{18} \mathrm{O}_{\mathrm{W}}\right)+16.9+\mathrm{C}(\mathrm{p})$

The function $\mathrm{C}$ is determined from a linear regression of true potential temperature against temperature in the Fukumori et al. atlas (1991). This simplified relation between potential temperature and temperature seems adequate for our purpose (see Figure IV-4). $\delta^{18} \mathrm{O}_{\mathrm{W}}$ is assumed to be a constant, i.e. the $\delta^{18} \mathrm{O}_{\mathrm{W}}$ signal is assumed to be uniform over the entire ocean (which would be an acceptable approximation if it were solely due to ice volume variations). Replacing $\delta^{18} \mathrm{O}_{\mathrm{W}}$ by a constant does not introduce any error in the potential temperature conservation equation:

$$
\sum_{i} U_{i} \times \Theta_{i}=0
$$

because $\delta^{18} \mathrm{O}_{\mathrm{W}}$ is a conservative tracer:

$$
\sum_{\mathrm{i}} \mathrm{U}_{\mathrm{i}} \times \delta^{18} \mathrm{O}_{\mathrm{w}}=0
$$




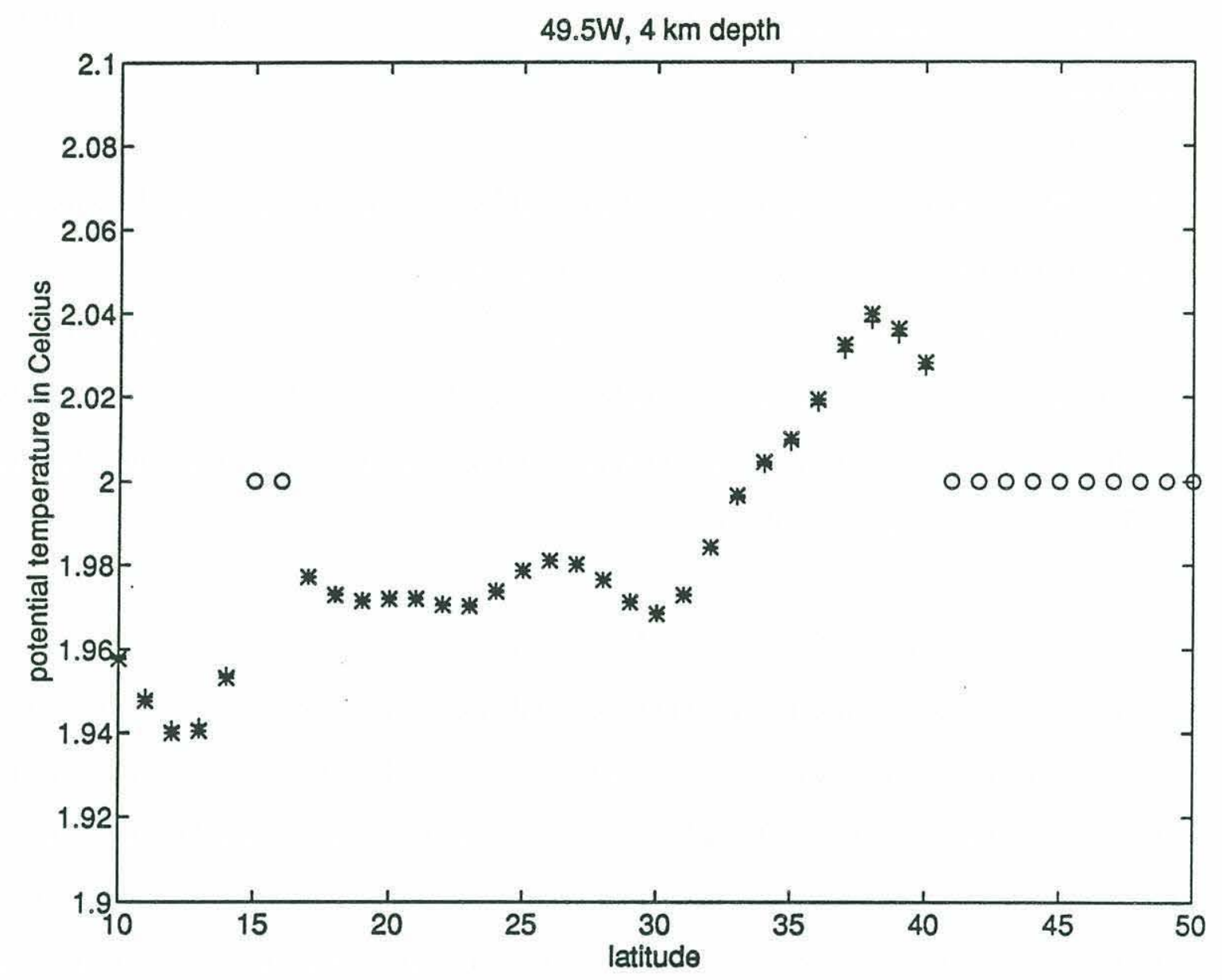

Figure IV-4: Stars represent potential temperature from the Fukumori et al. atlas (1991) at $49.5^{\circ} \mathrm{W}, 4 \mathrm{~km}$ depth. Crosses represent potential temperature estimated using the relation: $\Theta=T+C(p)$ and the Fukumori et al. temperature field at $49.5^{\circ} \mathrm{W}, 4$ $\mathrm{km}$ depth. The two estimates nearly coincide. Circles represent locations where ocean is shallower than $4 \mathrm{~km}$ depth. 
It does introduce, however, an error in the thermal wind equations because:

$$
\begin{aligned}
\nabla_{\mathrm{h}} \rho & =\alpha \times \nabla_{\mathrm{h}} \mathrm{T}+\beta \times \nabla_{\mathrm{h}} \mathrm{S} \\
& =-4.0 \times \alpha \times \nabla_{\mathrm{h}}\left(\delta^{18} \mathrm{O}_{\mathrm{c}}-\delta^{18} \mathrm{O}_{\mathrm{w}}\right)+\beta \times \nabla_{\mathrm{h}} \mathrm{S}
\end{aligned}
$$

where $\nabla_{\mathrm{h}}$ denotes the horizontal gradient operator, and $\alpha, \beta$ are the coefficients of the linear equation of state (see below). However, salinity, which is unknown for the LGM, is constrained to be such that $\nabla_{\mathrm{h}} \mathrm{S} \approx 0$ (see section $\mathrm{V}-\mathrm{B}-1$ ) which is a much larger source of error than assuming $\nabla_{\mathrm{h}} \delta^{18} \mathrm{O}_{\mathrm{w}} \approx 0$. Thus, the interpretation of $\delta^{18} \mathrm{O}_{\mathrm{c}}$ data in terms of potential temperature is reasonable in view of the other approximations introduced in the model.

In the LGM ocean, there are no phosphate data, and we use $\delta^{13} \mathrm{C}$ data instead. One can clearly recognize the different water masses in a meridional section of $\delta^{13} \mathrm{C}$ (see chapter II), and, like phosphate, $\delta^{13} \mathrm{C}$ can be considered as an almost conservative tracer in the North Atlantic. In the absence of biology, conservation equations for ${ }^{13} \mathrm{C}$ and ${ }^{12} \mathrm{C}$ can be written in each box as:

$$
\begin{aligned}
& \text { 1) } \sum_{\mathrm{i}} \mathrm{U}_{\mathrm{i}} \times 13 \mathrm{C}_{\mathrm{i}} \approx 0 \Rightarrow \sum_{\mathrm{i}} \mathrm{U}_{\mathrm{i}}\left(1+\frac{\delta 13 \mathrm{C}_{\mathrm{i}}}{1000}\right) \times 12 \mathrm{C}_{\mathrm{i}} \approx 0 \\
& \text { 2) } \sum_{\mathrm{i}} \mathrm{U}_{\mathrm{i}} \times 12 \mathrm{C}_{\mathrm{i}} \approx 0 \\
& \text { (1) }-2)\} \times 1000 \Rightarrow \sum_{\mathrm{i}} 12 \mathrm{C}_{\mathrm{i}} \times \mathrm{U}_{\mathrm{i}} \times \delta 13 \mathrm{C}_{\mathrm{i}} \approx 0
\end{aligned}
$$

Typically, ${ }^{12} \mathrm{C}=\mathrm{O}(2000) \pm 50 \mu \mathrm{mol} / \mathrm{kg}$, at least locally. In the model, we assume that it is a constant over each box so carbon 13 conservation equations can be rewritten as:

$$
\sum_{\mathrm{i}} \mathrm{U}_{\mathrm{i}} \times \delta 13 \mathrm{C}_{\mathrm{i}} \approx 0
$$

Note that taking into account the effect of ${ }^{12} \mathrm{C}$ variations, as well as that of biological particle fluxes, would result in a larger number of poorly known parameters, and reduce the capability of paleo-data to constrain the oceanic circulation. 


\section{c/ Thermal wind}

These relations can be derived from the geostrophic relations (relations 1) and 2)) and the hydrostatic balance (relation 3)):
1) $f v=1 / \rho_{0} \frac{\partial p}{\partial x}$
2) $f u=-1 / \rho_{0} \frac{\partial p}{\partial y}$
3) $\frac{\partial \rho}{\partial z}=-\rho g$
$\Rightarrow\left\{\begin{array}{c}\mathrm{f} \frac{\partial \mathrm{v}}{\partial z}=-g / \rho_{0} \frac{\partial \rho}{\partial x} \\ \mathrm{f} \frac{\partial \mathrm{u}}{\partial z}=g / \rho_{0} \frac{\partial \rho}{\partial y}\end{array}\right\}$ thermal wind

where $\mathrm{x}, \mathrm{y}$ and $\mathrm{z}$ are the coordinates along the longitude, latitude and vertical axis, $\mathrm{f}$ is the coriolis parameter, $\rho_{0}$ is a reference density, $u, v$, and $w$ are the zonal, meridional and vertical components of the velocity vector, and $p$ is the pressure. $\rho$ is the in situ density and is different from $\rho_{0}$ (reference density taken to be $1025 \mathrm{~kg} / \mathrm{m}^{3}$ in the model). Horizontal density gradients are evaluated along surfaces of constant pressure, or, almost equivalently, along surfaces of constant depth. The terminology "thermal wind" comes from meteorology. Indeed, for a perfect gas:

$$
\mathrm{P} \rho=\mathrm{rT}
$$

and, thus:

$$
\frac{\partial \rho}{\partial x}=\frac{r}{p \partial x} \text { and } \frac{\partial \rho}{\partial y}=\frac{r}{p \partial y}
$$

on surfaces $\mathrm{P}=$ constant. Thus, the thermal wind relations link the vertical variations of the horizontal component of the velocity field to the tilt of the isotherms in the atmosphere or the tilt of the isopycnals in the ocean. In the model, these relations are approximated using finite difference derivatives:

$$
\begin{aligned}
& U_{t}-U_{b}=\frac{g}{\rho_{0} f} \Delta z^{2}\left(\rho_{n}-\rho_{s}\right) \\
& V_{t}-V_{b}=\frac{-g}{\rho_{0} f} \Delta z^{2}\left(\rho_{e}-\rho_{w}\right)
\end{aligned}
$$

where $\mathrm{U}_{\mathrm{t}}, \mathrm{U}_{\mathrm{b}}$ are zonal transports, and $\mathrm{V}_{\mathrm{t}}, \mathrm{V}_{\mathrm{b}}$ are meridional transports in the top and bottom layers. $\rho_{\mathrm{n}}, \rho_{\mathrm{S}}, \rho_{\mathrm{e}}$, and $\rho_{\mathrm{w}}$ are the densities at the interface between the upper and the lower boxes (Figure IV-5). 


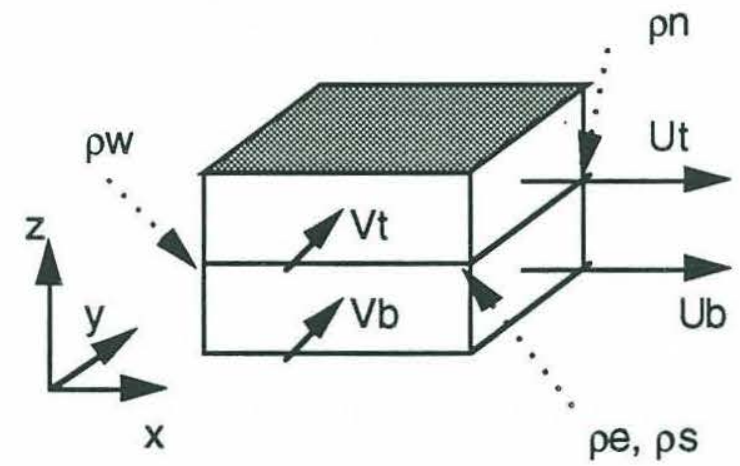

Figure IV-5: The vertical shear of the horizontal components of the velocity vector is linked to the horizontal gradients of density. The D-grid is the most natural grid for the discretization of the thermal wind balance

The contributions of the thermal wind balance to the objective function are of the type:

$$
\begin{aligned}
& \mathrm{J}_{\mathrm{TW}_{\mathrm{U}}}=\left(\mathrm{U}_{\mathrm{t}}-\mathrm{U}_{\mathrm{b}}-\frac{\mathrm{g}}{\rho_{0} \mathrm{f}} \Delta \mathrm{z}^{2}\left(\rho_{\mathrm{n}}-\rho_{\mathrm{s}}\right)\right)^{2} \\
& \mathrm{~J}_{\mathrm{TW}_{\mathrm{v}}}=\left(\mathrm{V}_{\mathrm{t}}-\mathrm{V}_{\mathrm{b}}+\frac{\mathrm{g}}{\rho_{0} \mathrm{f}} \Delta \mathrm{z}^{2}\left(\rho_{\mathrm{e}}-\rho_{\mathrm{w}}\right)\right)^{2}
\end{aligned}
$$

Small ageostrophic transports are possible, and relatively low weights are put on the minimization of JTWu and JTWv. Moreover, the thermal wind balance is not imposed in presence of topographic walls (Figure IV-6). Because there is no flow through these walls, imposing thermal wind would result in flat isopycnals along topographic features, which is unrealistic.
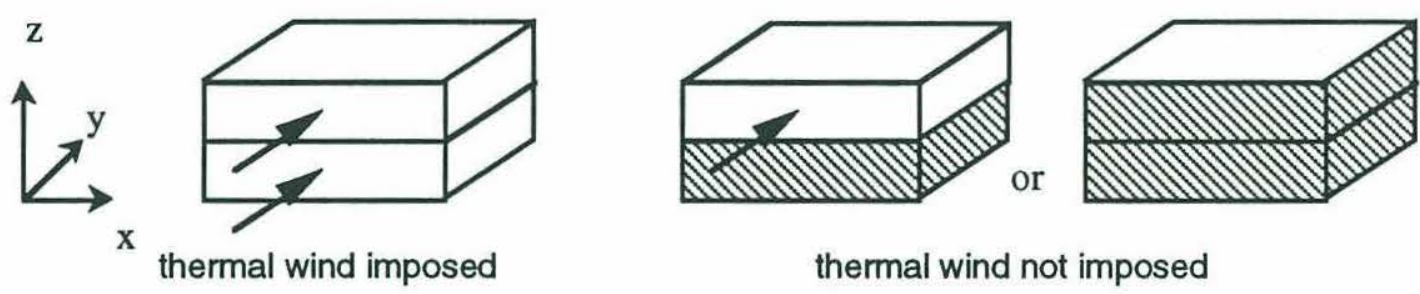

thermal wind not imposed

Figure IV-6: The thermal wind balance is not imposed in presence of walls

\section{d/ Linear vorticity balance}

The other dynamical constraint used in the model is the linear vorticity balance which is derived from the geostrophic relations (relations 1) and 2)), like the thermal wind balance, and from the mass conservation equations (relation 3). 

1) $f v=1 / \rho_{0} \frac{\partial p}{\partial x}$
2) $f u=-1 / \rho_{0} \frac{\partial p}{\partial y}$
3) $\frac{\partial u}{\partial x}+\frac{\partial v}{\partial y}+\frac{\partial w}{\partial z}=0$

Adding the partial derivative with respect to $y$ of the first equation and the partial derivative with respect to $\mathrm{x}$ of the second equation, and using the third equation, one obtains:

$$
\text { 4) } \beta v=f \frac{\partial w}{\partial z}
$$

The notations are the same as in paragraph c); Relation 4) is the linear vorticity balance; $\beta$ is the derivative of $f$ with respect to $y$. This equation can be rewritten as the conservation of potential vorticity along streamlines for a water column of infinitesimal height $\mathrm{h}$ :

$$
\text { 5) } \frac{\mathrm{d}}{\mathrm{dt}}\left(\frac{\mathrm{f}}{\mathrm{h}}\right)=0 \quad \text { where } \frac{\mathrm{d}}{\mathrm{dt}}=\mathrm{u} \cdot \nabla_{\mathrm{h}}
$$

is the horizontal derivative following a parcel of water. Thus, one can think of the linear vorticity balance as the conservation of a dynamical tracer: potential vorticity.

On the finite difference grid, $\partial w / \partial z$ is approximated by: $\left(w_{t}-w_{b}\right) / \Delta z$, and $\beta v / f$ is taken as the mean of $\beta_{S} v_{s} / f_{S}$ and $\beta_{n} v_{n} / f_{n}$, where the indices $s, n, t$, and $b$ designate the southern, northern, top, and bottom sides of the box (Figure IV-7). 


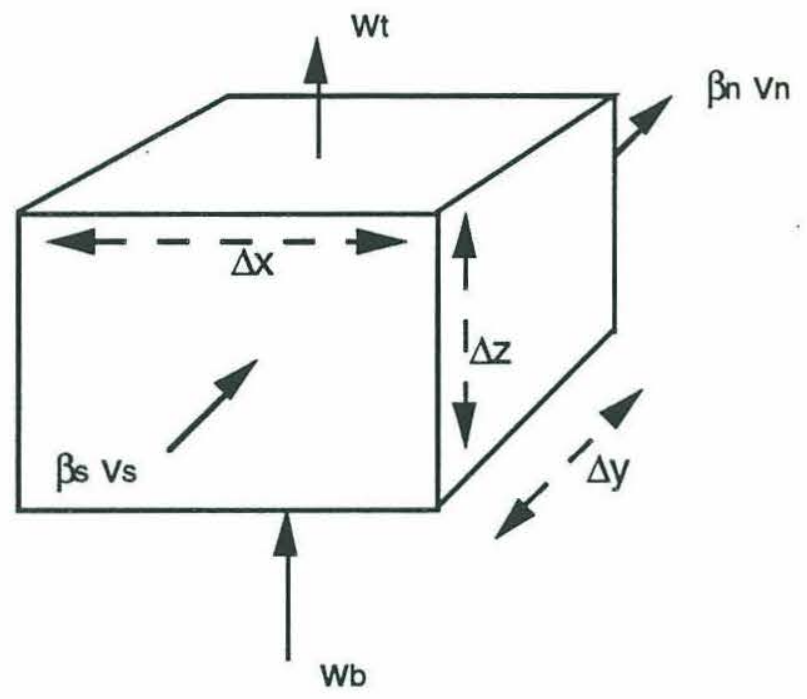

Figure IV-7: $\beta \mathrm{v}$ is taken to be the average of the advection of planetary vorticity through the southern and northern boundaries. $v_{\mathbf{S}}$ and $v_{n}$ are the meridional velocities across the southern and northern sides of the box. $w_{b}$ and $w_{t}$ are the vertical velocities across the bottom and top of the box.

The vorticity equation can be written in a finite difference form as:

$$
\frac{1}{2}\left(\frac{\beta_{s}}{f_{s}} V_{s}+\frac{\beta_{n}}{f_{n}} V_{n}\right)=W_{t}-W_{b}
$$

where $\mathrm{V}_{\mathrm{S}}=\mathrm{v}_{\mathrm{S}} \times \Delta \mathrm{x}_{\mathrm{S}} \times \Delta \mathrm{z}, \mathrm{V}_{\mathrm{n}}=\mathrm{v}_{\mathrm{n}} \times \Delta \mathrm{x}_{\mathrm{n}} \times \Delta \mathrm{z}, \mathrm{W}_{\mathrm{t}}=\mathrm{w}_{\mathrm{t}} \times \Delta \mathrm{x} \times \Delta \mathrm{y}$, and $\mathrm{W}_{\mathrm{b}}=\mathrm{w}_{\mathrm{b}} \times \Delta \mathrm{x} \times \Delta \mathrm{y}$ are the volume transports across the sides of the box. $\Delta \mathrm{x}_{\mathrm{S}}$ and $\Delta \mathrm{x}_{\mathrm{n}}$ are the widths of the box at the southern and the northern boundaries. $\Delta \mathrm{x}$ is the average of $\Delta \mathrm{x}_{\mathrm{S}}$ and $\Delta \mathrm{x}_{\mathrm{n}}$. The contribution of the linear vorticity balance to the objective function is of the type:

$$
J_{L V}=\left[\frac{1}{2}\left(\frac{\beta_{s}}{f_{s}} V_{s}+\frac{\beta_{n}}{f_{n}} V_{n}\right)-\left(W_{t}-W_{b}\right)\right]^{2}
$$

The finite difference version of the linear vorticity equation is only an approximation to the true relation. Thus, a small weight is put on the minimization of $\mathrm{J}_{\mathrm{LV}}$. The linear vorticity balance is imposed everywhere, except in the Deep Western Boundary Current where we do not expect it to hold (Stommel and Arons, 1960a\&b). This balance is not imposed in presence of zonal topography either (Figure IV-8). 


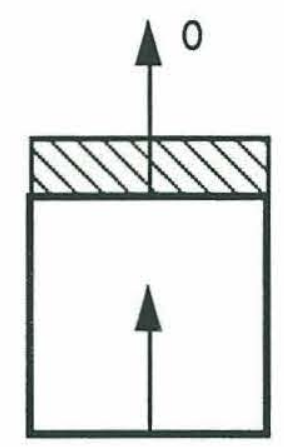

ßs Vs

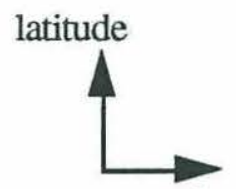

longitude

Figure IV-8: The linear vorticity balance is not imposed since there is a zonal wall on one side of the box.

Indeed, in the real ocean, topography is not a vertical wall, and there should be some meridional flow across the box interface. The condition of no-meridional flow across zonal topography must be imposed, however, in order to conserve mass in the model (otherwise, there would be a flux of mass leaving the model domain). If combined with the linear vorticity balance, this condition results in some unjustified constraints on the vertical transports. Also, the thermal wind equations are not imposed in the model near topographic walls, which means that the flow is not in geostrophic balance there. It would be somewhat inconsistent to impose the linear vorticity equation in an area where the geostrophic balance is not imposed.

\section{e/ Linear equation of state}

As mentioned earlier, both potential temperature and salinity are treated in the model as variables. For the sake of simplicity, density has been replaced in the thermal wind relations by its expression as a function of temperature and salinity obtained from a linearized equation of state. The coefficients $\alpha$ and $\beta$ of this equation where determined every $\mathrm{km}$ between 1 and $5 \mathrm{~km}$ from a linear regression of data from the Fukumori et al. atlas (1991) at these 5 different depths:

$$
\nabla \rho=\alpha(\mathrm{k}) \times \nabla \mathrm{T}+\beta(\mathrm{k}) \times \nabla \mathrm{S} \quad \mathrm{k}=1: 5
$$

where $\rho, T$, and $S$ designate density in $\mathrm{kg} / \mathrm{m}^{3}$, temperature in ${ }^{\circ} \mathrm{C}$, and salinity in PSS. The values of these coefficients are given in Table IV-1. Figure IV-9 shows that the approximate density variation computed using the linearized equation of state is reasonably close to the true density variation. The introduction of a fully non-linear equation of state would make the coding 


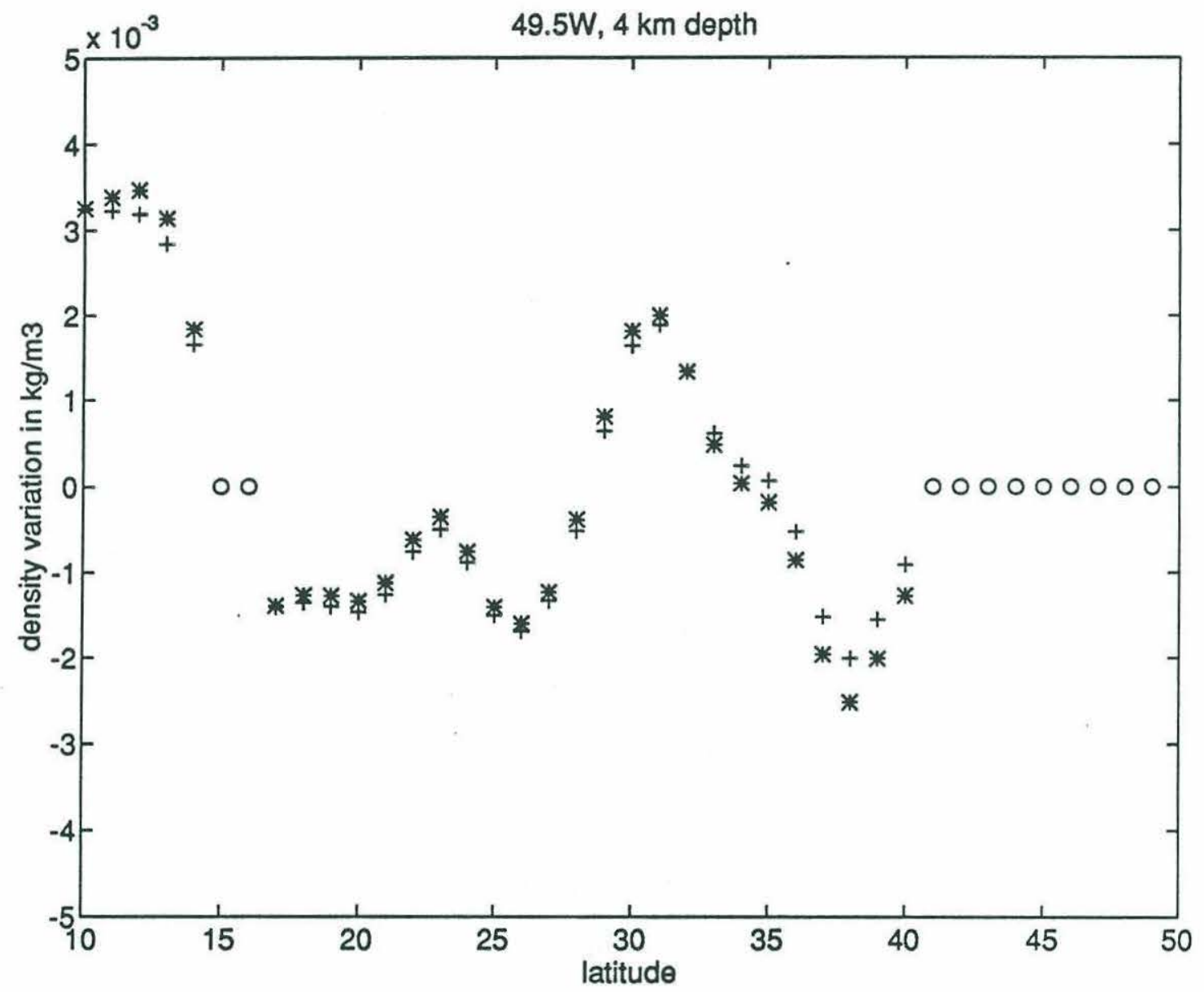

Figure IV-9: Stars represent the density variations (density minus mean density over the $49.5^{\circ} \mathrm{W}, 4 \mathrm{~km}$ depth line) at $49.5^{\circ} \mathrm{W}, 4 \mathrm{~km}$ depth in the Fukumori et al. (1991) atlas, as a function of latitude. Crosses represent density variations estimated from temperature and salinity, using the linear equation of state. Circles represent locations where ocean is shallower than $4 \mathrm{~km}$ depth. 


\begin{tabular}{|c|c|c|}
\hline depth $(\mathrm{km})$ & $\alpha$ & $\beta$ \\
\hline 1 & -0.1426 & 0.6754 \\
\hline 2 & -0.1529 & 0.7806 \\
\hline 3 & -0.1691 & 0.7703 \\
\hline 4 & -0.1878 & 0.7317 \\
\hline 5 & -0.2090 & 0.7381 \\
\hline
\end{tabular}

Table IV-1: coefficients of the linear equation of state 9

of the thermal wind balance more complicated. The linearized equation allows us to replace directly density by the associated potential temperature and salinity. Moreover, it makes the minimization of the imbalance in the thermal wind relation relatively easy because $\mathrm{J}_{\mathrm{TWu}}$ and $\mathrm{J}_{\mathrm{TWv}}$, the contributions of thermal wind to the cost function in each box, are simple quadratic functions which can be easily minimized. The fully non-linear equation of state is extremely complicated, and would probably introduce some difficulties such as local minima in the objective function. However, there are no fundamental reasons that require the use of a linearized state equation; the technical difficulties could be overcome if necessary.

The set of equations which governs the physical constraints of the model are very similar to the so-called "thermocline equations" (Pedlosky, 1986). The flow is in geostrophic and hydrostatic balance, and conserves mass and potential density (since potential temperature and salinity are conserved). These equations constitute the basic rules governing the ocean circulation. Adding more sophisticated physics would not fundamentally alter the behavior of the model. Since they are not an exact representation of the ocean's physics and they are modelled by finite difference approximations on a very coarse resolution grid, small imbalances are tolerated in these equations. Section IV-5 discusses these imbalances and the criteria that determine the degree to

\footnotetext{
${ }^{9}$ The values of $\beta$ appear to be too small, especially at $1 \mathrm{~km}$ depth (see Gill, 1982). These low values, however, are consistent with more plausible values of about $0.8 \mathrm{~kg} / \mathrm{m}^{3} / \mathrm{PSS}$ within the uncertainty of the linear regression (about $0.1 \mathrm{~kg} / \mathrm{m}^{3} / \mathrm{PSS}$ ). This large uncertainty is probably due to the small range of salinities in the deep ocean. Note that this imprecision on the values of $\beta$ does affect significantly the solution (the numerical errors due to the very coarse resolution of the model are probably much larger), and does not play a role in the results presented in the next chapter.
} 
which they are reasonable. Tracer conservation equations $\left(\Theta, \mathrm{S}\right.$, and ${ }^{13} \mathrm{C}$ or $\left.\mathrm{PO} 4\right)$ are necessarily non-linear because both transports and tracer concentrations are variable. These non-linear constraints are technically more difficult to handle than the linear ones, but also provide a quantitative link between the circulation of the ocean and its chemical properties. The thermal wind relation is the fundamental tool to interpret hydrographic data. However, its application to paleoclimate studies is less straightforward because of the difficulty of extracting the temperature signal from $\delta^{18} \mathrm{O}$ data and the impossibility, until now, of reconstructing paleosalinities in the deep ocean. The thermal wind should not be overlooked, however, because it guarantees the model solution is in geostrophic balance. Moreover, Wunsch (1985) has shown that providing a model with direct information about the velocity field greatly constrains the solution. The thermal wind balance linearly links the circulation to density, which is a partly measurable quantity, and thus provides direct information about the velocities.

\section{4) Data constraints}

This section explains how the physical constraints described above are combined with the data to produce a "best estimate" of the ocean circulation.

By "best estimate", we mean a solution which satisfies the physical and observational constraints as well as possible in a least square sense. It is the solution $\mathbf{x}$ for which the objective function:

$$
J=\left(\mathbf{x}-\mathbf{x}_{\mathbf{0}}\right)^{\mathrm{T}} \mathrm{C}_{\mathbf{0}}^{-1}\left(\mathbf{x - x _ { 0 }}\right)+\mathbf{f}(\mathbf{x})^{\mathrm{T}} \mathrm{C}_{\mathbf{f}}^{-1} \mathbf{f}(\mathbf{x})
$$

is a minimum; $\mathbf{x}$ is the state vector containing all the variables, $\mathbf{x}_{\mathbf{0}}$ is the a priori estimate of $\mathbf{x}$, $\mathbf{f}(\mathbf{x})$ contains the residuals of the physical constraints, and $\mathbf{C}_{\mathbf{0}}^{-1}, \mathbf{C}_{\mathbf{f}}^{-1}$ are weight matrices (see chapter III). The first part of the objective function represents the data misfit. Note that, in this problem, the observations directly constrain the elements of the state vector. In the modern ocean simulation, the "observations" are in fact tracer distributions obtained from a hydrographic atlas. Tracer values are subsampled at each model grid point, and thus directly correspond to elements of the state vector. In glacial ocean-simulations, observations of the isotopic composition of foraminifera shells are assigned to neighboring grid-points. A more general approach consists of 
treating the observations as a constraint on a linear combination of elements of the state vector. In this case, $\mathbf{x}-\mathbf{x}_{\mathbf{0}}$ is replaced in the data misfit term by an expression of the form $\mathbf{E x}-\mathbf{y}$, where $\mathbf{E}$ is a matrix, and $\mathbf{y}$ represents the observations. For instance, the observations $\mathbf{y}$, can constrain a weighted mean of neighboring tracer grid-points, Ex. In the glacial ocean, there are few data points in the Western Atlantic and in the intermediate layers of the Atlantic, and it was judged more practical to subjectively assign the observations to model grid-points rather than using an explicit interpolation scheme.

Since the data are noisy, the data misfit need not be zero. The magnitude of $\left(\mathbf{x}-\mathbf{x}_{\mathbf{0}}\right)$ after inversion will depend on the weight matrix $\mathbf{C}_{\mathbf{0}}{ }^{-1}$. Ideally, $\mathbf{C}_{\mathbf{0}}$ is the covariance matrix of the $a$ priori estimate of the solution $\mathbf{x}_{\mathbf{0}}$. Its diagonal terms tell how much we trust data points and the non diagonal terms indicate the correlation between the various data misfits.

For the last glacial maximum, the a priori values of the tracers at many grid points have to be guessed at, and it is not clear what the error bars and the cross-correlations should be. The problem is even more difficult for the transport variables: we do not know at all what the circulation should be and even less its statistics. In these circumstances, it seems justified to use a simple diagonal matrix to approximate the covariance of the a priori solution. When observations are available, the corresponding diagonal terms are set to be the variance (the square of the standard error) of the observations. When no observation is available, a large error bar is attributed to the a priori guess of the variable, so that it does not constrain the solution. However, the error bar cannot be infinitely large because of numerical problems. Indeed, $\mathrm{C}_{\mathbf{0}}{ }^{-1}$, which is basically the square of the inverse of the error bar, must be large enough to eliminate the smallest singular values associated with the physical constraints (see Mercier et al., 1993, for a discussion of this problem).

For the modern ocean, it is possible to estimate the covariance $\mathbf{C}_{\mathbf{0}}$ from the data. The tracer data used in the model are potential temperature, salinity, and phosphate from the objectively mapped atlas of Fukumori et al. (1991). Objective mapping is a procedure to map irregularly distributed data, such as hydrographic data, on a regular grid using explicit information concerning the data statistics. It also provides an estimate of the uncertainty of the mapped field. There is usually less uncertainty where there is good sampling of the data. For the sake of 
simplicity, we use averaged estimates of the uncertainty over the whole North Atlantic at model depths 1-5 km following Fukumori et al (1991). The values given in their atlas are interpolated in order to make them correspond to the standard depths of the model (in the atlas, uncertainty estimates are given at 700, 1200, 2000, 3250, 4500 meters; see Table IV-2 for the interpolated values).

\begin{tabular}{|c|c|c|c|}
\hline depth $(\mathrm{km})$ & pot. temp. $\left({ }^{\circ} \mathrm{C}\right)$ & salinity $(\mathrm{PSS})$ & phosphate $(\mu \mathrm{mol} / \mathrm{l})$ \\
\hline 1 & 0.6 & 0.1 & 0.1 \\
\hline 2 & 0.2 & 0.02 & 0.05 \\
\hline 3 & 0.05 & 0.005 & 0.03 \\
\hline 4 & 0.05 & 0.005 & 0.05 \\
\hline 5 & 0.05 & 0.005 & 0.06 \\
\hline
\end{tabular}

Table IV-2: Standard errors, estimated from the Fukumori et al. atlas

The covariance matrix $\mathbf{C}_{\mathbf{o}}$ is taken to be diagonal, and its elements are the square of the data standard errors given in Table IV-2. Modern ocean tracer data are taken from the Fukumori et al. atlas. Potential temperature, salinity, and phosphate are subsampled every $10^{\circ}$ in the horizontal and every kilometer in the vertical. The standard deviations described in Table IV-2 are probably too small because they do not include the error introduced by subsampling. However, it is not clear how large this subsampling error is, so we did not take it into account. Where there are no data because a model grid point falls on land (the $10^{\circ}$ grid cannot closely follow the coast or the topography, and some grid points correspond to land areas in the real world), we take the closest data point in the ocean or simply extrapolate in the few cases when there is no such point nearby.

Another issue is the choice of data points representing the Deep Western Boundary Current. Had we simply sampled the data at the exact location of the western boxes, we would have missed the gradients of density associated with the western intensification. We picked the Deep Western Boundary Current data in order to have the maximum density jump across the current. Figures IV$10-a \& b$ illustrate this idea: 


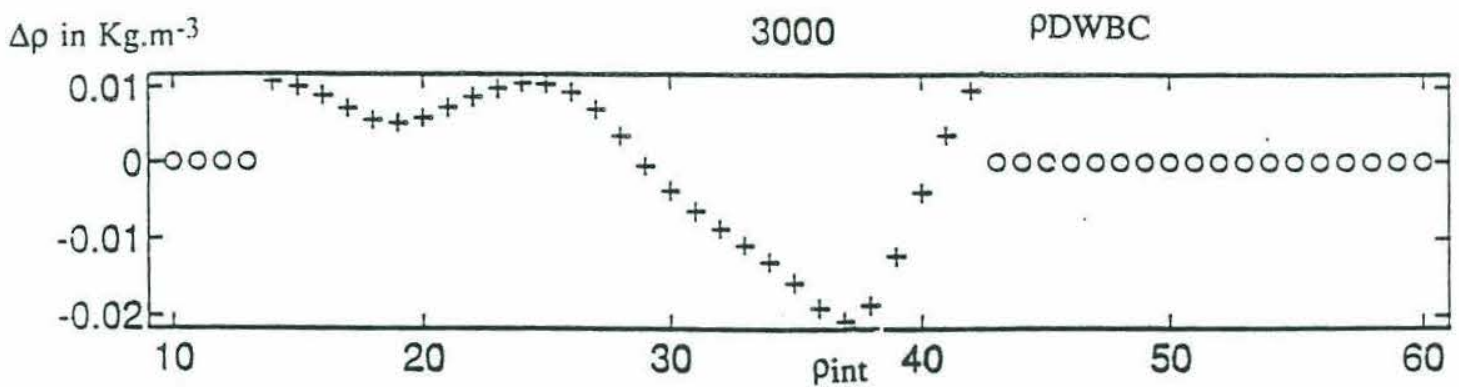

Figure IV-10-a: Density at $3 \mathrm{~km}$ depth, $59.5^{\circ} \mathrm{W}$, from the Fukumori et al atlas (1991). Axes are latitude in degrees, and density in $\mathrm{Kg} . \mathrm{m}^{-3}$ (mean density over line subtracted). Circles represent land areas. In the model, the temperature and salinity data points are picked so that they maximize the density jump across the DWBC. $\rho$ int and $\rho D W B C$ are picked at $37^{\circ} \mathrm{N}$ and $42^{\circ} \mathrm{N}$ (see Figure IV-10-b for definition of $\rho_{\text {int }}$ and $\rho D W B C$ ).

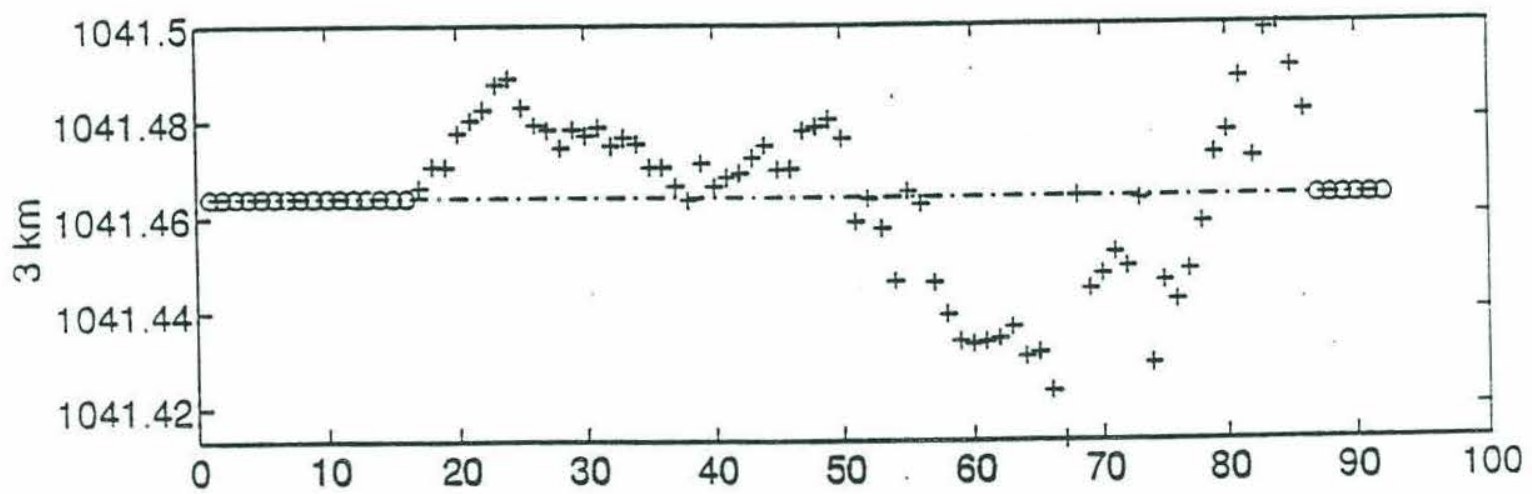

Figure IV-11: In situ density at $3 \mathrm{~km}$ depth at $64^{\circ} \mathrm{W}$ from Endeavor 129 cruise (see Fukumori et al. (1991) for cruise track). Axes are station numbers and density in Kg.m-3. Circles represent land areas. 


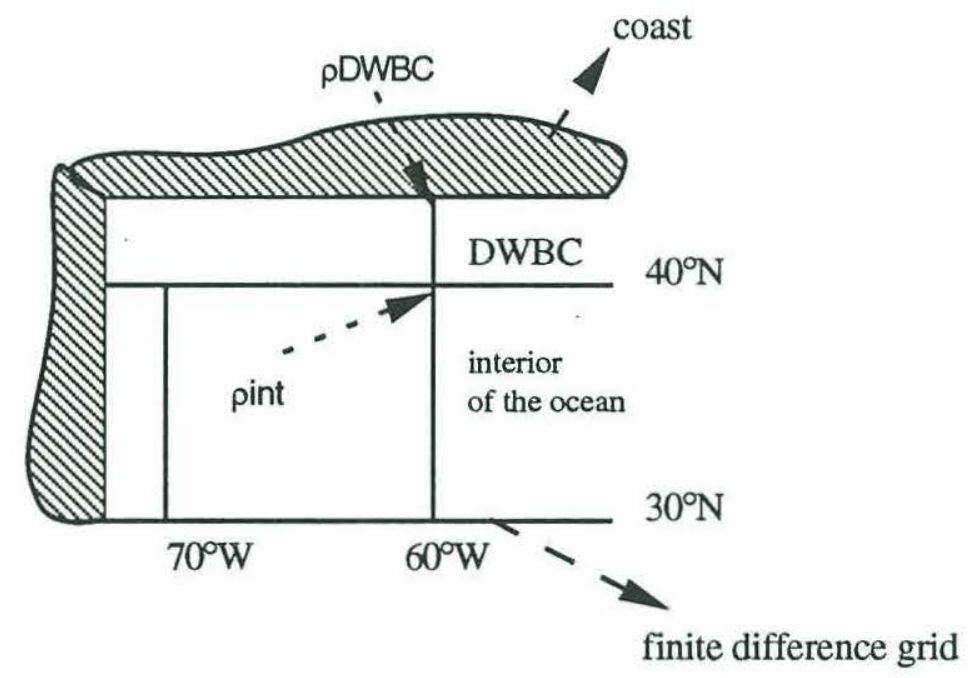

Figure IV-10-b: PDWBC is the density onshore of the DWBC; $\rho_{\text {int }}$ is the density at the interface between the DWBC and the interior of the ocean. This interface is formally at $40^{\circ} \mathrm{N}$ in the model. In practice, we sample $\rho_{\text {int }}$ so that $P D W B C-\rho$ int is a maximum.

If we take $\rho_{\text {int }}$ to be the density at $40^{\circ} \mathrm{N}$, and $\rho_{\mathrm{dwbc}}$ the density at $42^{\circ} \mathrm{N}\left(42^{\circ} \mathrm{N}\right.$ is the position of the continental margin, see Figure IV-10-b for definition of $\rho_{\text {int }}$ and $\rho_{\mathrm{d} w b c}$ ), we get a change of density of $10^{-2} \mathrm{~kg} \cdot \mathrm{m}^{-3}$ across the Deep Western Boundary Current at a depth of $3 \mathrm{~km}$ and a longitude of $59.5^{\circ} \mathrm{W}$ (Figure IV-10-a). However, the steep gradient of density characterizing the DWBC extends to $37^{\circ} \mathrm{N}$ (Figure IV-10-a), so it seems logical to assign the $37^{\circ} \mathrm{N}$ data to the model grid point. Then, we obtain a change of density across the DWBC of $3 \times 10^{-2} \mathrm{~kg} \cdot \mathrm{m}^{-3}$.

The idea behind taking the largest possible gradient of density is to try to compensate for the smoothing of the density field introduced by the objective mapping procedure. Indeed, even after doing so, we still get some sections where there is not much of a density gradient. The effect of smoothing is clearly seen by comparing a density section from the atlas (Figure IV-10-a) with raw hydrographic data near $60^{\circ} \mathrm{W}$ (Figure IV-11). The density gradient in the Deep Western Boundary Current at $60^{\circ} \mathrm{W}$ obtained from the atlas (without subsampling) is about half that obtained from the "Endeavor 129" cruise at a similar longitude, even though these cruise data are included in the atlas. The larger density gradient in the Endeavor data could be due to an eddy which would have been filtered out by the objective mapping procedure. However, the same observation can be made in other sections, and it is unlikely that the same eddy could show up in several 
hydrographic sections. Note that the smoothing effect would probably be worse if we had used the Levitus atlas (Levitus, 1982) because its decorrelation scale is larger.

Since the procedure used to subsample data in the Deep Western Boundary Current is somewhat debatable, we take the error bars associated with these data to be twice the ones given in Table IV-2. We also double the Table IV-2 values for the error bars assigned to grid points corresponding to land areas. We multiply by 4 the Table IV-2 error bars for data extrapolated when there are no neighboring oceanic point.

For the mass flux variables, we take as an a priori guess the transports computed from the Fukumori et al. potential temperature and salinity fields, assuming a reference level at $3 \mathrm{~km}$ depth or the bottom of the ocean if shallower. In the DWBC, the a priori estimate is adjusted so that the vertically integrated transport below $1 \mathrm{~km}$ depth is $15 \mathrm{~Sv}$. Since a small shift in the reference velocity can result in a large change in the integrated transports, we put a large, $5 \mathrm{~Sv}$, error bar on the $a$ priori estimate of the transports.

\section{5) How much imbalance in the physical constraints is acceptable?}

Physical constraints, with the exception of mass conservation, are not exactly satisfied. One way to decide whether a constraint imbalance is acceptable or not is to compare it to the model error due to simplified physics and numerical approximations (e.g. Wunsch, 1977; Mercier, 1989). However, it is not clear how important the subgrid scale processes are in the very coarse resolution model we use, and even less clear what the numerical error is. The equation imbalances should not be too large, otherwise the model physics will not be satisfied. At the same time, they should not be too small, otherwise the model will not be consistent with the observations. Thus, there is a trade off between the model physics and the observational constraints. Since the observational uncertainties are somewhat easier to quantify than the model error, we let the data misfit drift to its maximum acceptable value and try to obtain the smallest equation imbalances

possible. This result can be achieved by adjusting the weight matrix $\mathbf{C}_{\mathbf{f}}^{-1}$ in the objective function (see section IV-4 and chapter III). Note that the imbalances act as a source term in the conservation equations which could artificially widen the range of possible solutions. Because we 
are seeking solutions with very low imbalances, this artificial source term is small and the range of possible solutions we obtain is unlikely to be overestimated.

One difficulty is that a no flow solution is perfectly compatible with the model equations. Thus, when the model cannot find a consistent solution in a given box, it tends to reduce the imbalances by setting the transports into this box to zero. Such a no-flow solution is not satisfactory. Geostrophic calculations indicate that there is a net northward flow in the upper layers of the modern ocean (Hall and Bryden, 1982). This net northward mass transport must be compensated by a southern mass transport in the deep ocean. In other words, the geostrophic balance acts as a "clock" which sets the rate of overturning in the modern Atlantic, and is contradictory with a no-flow solution. This clock is not available in the glacial ocean because density gradients are unknown. $\Delta^{14} \mathrm{C}$ data indicate, however, that the planktonic-benthic foraminifera age difference was no more than a few hundred years in the Equatorial Atlantic during the LGM (Broecker et al., 1990). This age difference provides some information about the rate of overturning. in the glacial Atlantic. Indeed, deep waters are not in contact with the atmospheric source of ${ }^{14} \mathrm{C}$, and their ${ }^{14} \mathrm{C}$ content decreases because of radioactive decay. If the overturning slows down, the water masses are renewed less rapidly, and their age increases relative to the age of surface waters. If the overturning stops, the age difference between deep and surface waters becomes very large (though not infinite because fallout of organic matter acts as small source of $\left.{ }^{14} \mathrm{C}\right)$.

Thus, $\Delta^{14} \mathrm{C}$ plays the role of a clock in the glacial ocean similar to the geostrophic clock in the modern ocean. This clock tells us that the overturning did not stop during the Last Ice Age, and a no-flow solution is not acceptable. Note that passive tracers such as $\delta^{13} \mathrm{C}$ do not constitute such a clock. In the absence of biological activity, passive tracers are consistent with no-flow solutions. Also, one can multiply the fluxes of deepwater into an oceanic basin by any arbitrary constant without affecting the distribution of passive tracers. This point has important implications for the interpretation of passive tracer distributions in terms of oceanic circulation, that are discussed in chapter V.

Obtaining small equation imbalances does not prevent no-flow solutions because the imbalances vanish when the flow vanishes. Thus, a better criterion is needed to decide whether a 
solution is acceptable or not. Therefore, we introduce a quantity that we call equation residuals. It is defined as the ratio of the imbalance over the largest term in the equation. Physically, imbalances are meant to represent high order terms in the model equations, and should be small compared to the dominant terms. Thus, an imbalance will be tolerated if it is at least one order of magnitude smaller than the dominant term in the equation, i.e. if the equation residual is smaller than $10 \%$. If any equation residual is significantly larger than 0.1 , the solution is rejected. Noflow solutions usually result in small imbalances, but in large residuals because the largest term in the equation, which is the denominator of the residual, is as small as the imbalance. No-flow solutions can therefore be identified by looking at the residuals, and be rejected.

Note that the model will minimize the physical constraint imbalances, not their residuals. The residuals are mathematically more complicated than the imbalances so it is difficult to compute their derivatives. Also, their minimization is difficult because of their nonlinearity. Thus, the "residual" criterion is used only at the end of each run to compare different solutions in a systematic manner, and to decide when a solution should be rejected; but it is not explicitly included in the minimization procedure.

The remaining part of this section defines the residuals for each type of physical constraint.

\section{a/ Mass conservation}

Imbalances and residuals are defined as:

$$
\begin{aligned}
& \text { imbalance: } \mathrm{f}=\sum_{\mathrm{i}} \mathrm{U}_{\mathrm{i}} \\
& \text { residual: } \mathrm{res}=\frac{\sum_{\mathrm{i}} \mathrm{U}_{\mathrm{i}}}{\max \left(\mathrm{U}_{\mathrm{i}}\right)}
\end{aligned}
$$

where $U_{i}$ represents the mass transports going into the box and the normalization factor $\max \left(\left|U_{i}\right|\right)$ is the largest of these transports. In the model, the imbalances never exactly go to zero (partly because of numerical round-off errors), and an almost no-flow solution will usually result in a residual of order one. Because we want mass to be almost exactly conserved (see section IV-3-a), we put a large weight on the mass balance constraints so that their residuals after inversion be at most $10^{-4}$ or $0.01 \%$ everywhere and smaller than that on average. 


\section{b/ Tracer conservation}

The residuals of tracer conservation equations are defined in the same way as those of mass conservation equations:

$$
\begin{aligned}
& \text { imbalance: } f=\sum_{i} U_{i} \times C_{i} \\
& \text { residual: res }=\frac{\sum_{i} U_{i} \times C_{i}}{\max \left(U_{i} \times\left(C_{i}-C_{m}\right)\right)}
\end{aligned}
$$

$\max \left(\left|\mathrm{U}_{\mathrm{i}} \times\left(\mathrm{C}_{\mathrm{i}}-\mathrm{C}_{\mathrm{m}}\right)\right|\right)$ designates the largest flux of tracer entering or leaving the box, $\mathrm{C}_{\mathrm{m}}$ is the mean tracer concentration over the box (see Figure IV-12).

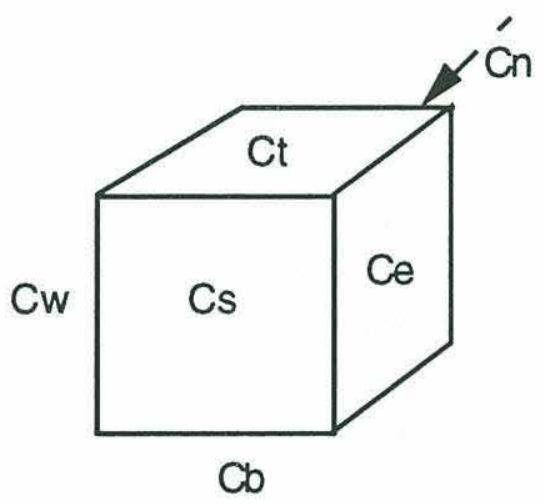

Figure IV-12: $\mathrm{Cm}=(\mathrm{Cw}+\mathrm{Ce}+\mathrm{Cs}+\mathrm{Cn}+\mathrm{Cb}+\mathrm{Ct}) / 6$

$\mathrm{Cm}$ is subtracted from $\mathrm{Ci}$ so that only the variations of the tracer concentrations within the box enter the normalization factor $\max \left(\left|\mathrm{U}_{\mathrm{i}} \times\left(\mathrm{C}_{\mathrm{i}}-\mathrm{C}_{\mathrm{m}}\right)\right|\right)$. Thus, for salt conservation for instance, the residuals are the same whether one expresses salinity using "PSS" or using "PSS - 35". Note that $\mathrm{C}_{\mathrm{m}}$ does not need to be subtracted from $\mathrm{C}_{\mathrm{i}}$ in the expression of the imbalances:

$$
\begin{aligned}
& \mathrm{f}=\sum_{\mathrm{i}} \mathrm{U}_{\mathrm{i}} \times \mathrm{C}_{\mathrm{i}}=\sum_{\mathrm{i}} \mathrm{U}_{\mathrm{i}} \times\left(\mathrm{C}_{\mathrm{i}}-\mathrm{C}_{\mathrm{m}}\right) \\
& \text { since } \sum_{\mathrm{i}} \mathrm{U}_{\mathrm{i}}=0 \text { (mass almost exactly conserved) }
\end{aligned}
$$

We will see when we look at the results of the modern ocean inversion that the largest tracer residuals are of the order of $10 \%$, and, on average, below $1 \%$. If a solution does not satisfy this criterion, it is rejected. The $10 \%$ upper bound is chosen so that the solution conserves tracers at 
least to the first order. Despite the simplified physics used in the model and the numerical approximations made, it is possible to find such a solution in the modern ocean (see part V-A).

\section{c/ Thermal wind balance}

These equations express the balance between two terms: the vertical shear of the horizontal components of the velocity field and the horizontal gradients of density; therefore, the residuals are defined as the sum of these two terms divided by the largest term:

$$
\text { residual: res }=\frac{\mathrm{f} \frac{\partial \mathrm{u}}{\partial \mathrm{z}}-\frac{\mathrm{g} \rho \rho}{\rho_{0} \partial \mathrm{y}}}{\max \left(\left|\mathrm{f} \frac{\partial \mathrm{u}}{\partial \mathrm{z}}\right|,\left|\frac{\mathrm{g} \partial \rho}{\rho_{0} \partial \mathrm{y}}\right|\right)} \text { and res }=\frac{\mathrm{f} \frac{\partial \mathrm{v}}{\partial \mathrm{z}}+\frac{\mathrm{g} \rho}{\rho_{0} \partial \mathrm{x}}}{\max \left(\left|\mathrm{f} \frac{\partial \mathrm{v}}{\partial \mathrm{z}}\right|, \mid \frac{\mathrm{g} \partial \rho}{\rho_{0} \partial \mathrm{x}}\right)}
$$

which translate numerically into:

$$
\text { residual: res }=\frac{\mathrm{U}_{\mathrm{t}}-\mathrm{U}_{\mathrm{b}}-\frac{\mathrm{g}}{\rho_{0} \mathrm{f}} \Delta \mathrm{z}^{2}\left(\rho_{\mathrm{n}}-\rho_{\mathrm{s}}\right)}{\max \left(\left|\mathrm{U}_{\mathrm{t}}-\mathrm{U}_{\mathrm{b}}\right|,\left|\frac{\mathrm{g}}{\rho_{0} \mathrm{f}} \Delta \mathrm{z}^{2}\left(\rho_{\mathrm{n}}-\rho_{\mathrm{s}}\right)\right|\right)} \text { and res }=\frac{\mathrm{V}_{\mathrm{t}}-\mathrm{V}_{\mathrm{b}}+\frac{\mathrm{g}}{\rho_{0} \mathrm{f}} \Delta \mathrm{z}^{2}\left(\rho_{\mathrm{e}}-\rho_{\mathrm{w}}\right)}{\max \left(\left|\mathrm{V}_{\mathrm{t}}-\mathrm{V}_{\mathrm{b}}\right|,\left|\frac{\mathrm{g}}{\rho_{0} \mathrm{f}} \Delta \mathrm{z}^{2}\left(\rho_{\mathrm{e}}-\rho_{\mathrm{w}}\right)\right|\right)} \text { see }
$$

section IV-3-c for the notation. The case $f \partial u / \partial z=g / \rho_{0} . \partial \rho / \partial y=0$ is physically possible but unlikely because, in the modern ocean simulation, the density gradients are constrained to stay close to their values prior to the inversion, and, in the ice age simulation, the transports are tightly constrained (see IV-6-a) so their shear cannot go to zero.

\section{d/ Linear vorticity balance}

The linear vorticity equation is a balance between the advection of planetary vorticity, $\beta v$, and vortex stretching, $\mathrm{f} \partial \mathrm{w} / \partial \mathrm{z}$. Thus, the residuals are normalized by the largest of these two terms:

$$
\text { residual: } \quad \text { res }=\frac{\beta v-f \frac{\partial w}{\partial z}}{\max \left(\left|\beta v, f \frac{\partial w}{\partial z}\right|\right)}
$$

$\beta v=f \partial w / \partial z=0$ is physically possible but unlikely, so the above expression is almost always usable.

The finite difference form of this residual is: 


$$
\text { residual: res }=\frac{\frac{1}{2}\left(\frac{\beta_{\mathrm{s}}}{\mathrm{f}_{\mathrm{s}}} \mathrm{V}_{\mathrm{s}}+\frac{\beta_{\mathrm{n}}}{\mathrm{f}_{\mathrm{n}}} \mathrm{V}_{\mathrm{n}}\right) \Delta \mathrm{z}-\left(\mathrm{W}_{\mathrm{t}}-\mathrm{W}_{\mathrm{b}}\right)}{\max \left(\left|\left(\frac{\beta_{\mathrm{s}}}{\mathrm{f}_{\mathrm{s}}} \mathrm{V}_{\mathrm{s}}+\frac{\beta_{\mathrm{n}}}{\mathrm{f}_{\mathrm{n}}} \mathrm{V}_{\mathrm{n}}\right) \Delta \mathrm{z}\right|,\left|\mathrm{W}_{\mathrm{t}}-\mathrm{W}_{\mathrm{b}}\right|\right)}
$$

see section IV-3-d for the notation.

The residuals are deemed acceptable if they are lower than $10 \%$. It is difficult to reduce them much below $10 \%$ without raising other residuals (those of the thermal wind equations for instance). Thus, we now have a tool for analyzing inversion results. We can reject solutions such as no flow solutions, which have large residuals. The residuals also allow for a systematic and quantitative comparison of different solutions.

\section{6) Problems associated with the non-linear inversion}

Solving non-linear inverse problems using optimization techniques is conceptually quite simple. However, the technical problems linked to the optimization of large systems and the formulation of an inverse model are far from being straightforward. This section reviews some of the difficulties we encountered: preventing the model from producing a no flow solution, getting reasonable transports in the Deep Western Boundary Current, finding the global minimum, and choosing the weight matrices of the cost function.

\section{a/ Preventing a no flow solution}

As mentioned above, all the model physical constraints are consistent with a no flow solution. Because we neglect the effect of biology, the ${ }^{13} \mathrm{C}$ or phosphate concentrations estimated by the model remain reasonable even if the circulation is very sluggish (because particulate matter is depleted in ${ }^{13} \mathrm{C}$ and contains phosphorus, biological activity would lower the $\delta^{13} \mathrm{C}$ and raise the phosphorus concentration of deep waters to unrealistic values in a sluggish ocean). Thus, one way for the model to reduce the equation imbalances is to set the circulation to zero. This can happen when two equations are contradictory. For instance, suppose we have incompatible mass and tracer conservation equations for a volume element of the model (Figure IV-13): 


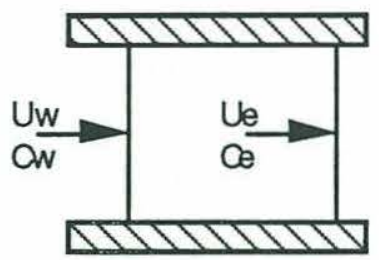

Figure IV-13: mass and tracer are conserved in this box

$$
\begin{aligned}
& \mathrm{U}_{\mathrm{w}}-\mathrm{U}_{\mathrm{e}}=0 \\
& \mathrm{U}_{\mathrm{w}} \mathrm{C}_{\mathrm{w}}-\mathrm{U}_{\mathrm{e}} \mathrm{C}_{\mathrm{e}}=0
\end{aligned}
$$

with:

$$
\mathrm{C}_{\mathrm{w}}=1 \pm 0.2, \mathrm{C}_{\mathrm{e}}=1.1 \pm 0.2 \text { and } \mathrm{U}_{\mathrm{e}}=\mathrm{U}_{\mathrm{w}}=1 \pm 5
$$

as an a priori guess of the solution to the equations. One obvious way to find a solution to the equations is to adjust $\mathrm{C}_{\mathrm{e}}$ so that $\mathrm{C}_{\mathrm{W}}=\mathrm{C}_{\mathrm{e}}=1$. However, the model would rather adjust the transport variables since they have a large uncertainty. Then the simplest solution for the model is to set $\mathrm{U}_{\mathrm{e}}$ and $\mathrm{U}_{\mathrm{w}}$ to 0 . Such a no-flow solution is rejected, however, because it results in large residuals, and we need to find a better solution.

In theory, no-flow solutions should not occur since the thermal wind balance imposes a vertical shear on the velocity field. However, since temperature and salinity can be modified, the model tends to flatten the isopycnals and thus reduce the shear. One way to prevent the shear from vanishing is to introduce horizontal correlations on the temperature and salinity fields by adding non diagonal terms in the covariance matrix of the a priori estimate of the solution, $\mathrm{C}_{\mathbf{0}}$. The hope is that the correlations will prevent low density values from going up and high density values from going down at the same time. However, this approach does not work using reasonable correlation coefficients, probably because, in the deep ocean, very small changes of temperature and salinity are sufficient to significantly modify the horizontal density gradients. Perhaps this method would be more efficient if we imposed the correlation on the density field itself, but this is difficult to do because density is not an explicit variable in the model.

A more successful approach, at least for modern ocean simulations, is to add a term in the objective function which penalizes the departure of density gradients from their a priori values:

$$
\mathrm{J}_{\nabla \rho}=\left(\nabla \rho-\nabla \rho_{0}\right)^{2}=\left[\alpha \times\left(\nabla \mathrm{T}-\nabla \mathrm{T}_{0}\right)+\beta \times\left(\nabla \mathrm{S}-\nabla \mathrm{S}_{0}\right)\right]^{2}
$$


where $\rho, \mathrm{T}$, and $\mathrm{S}$ are the density, temperature, and salinity variables; $\rho 0, \mathrm{~T}_{0}$, and $\mathrm{S}_{0}$ are their $a$ priori estimates; $\alpha$ and $\beta$ are the coefficients of the linear equation of state.This approach prevents the vertical shear from becoming zero. In theory, the same result could be achieved by tightly constraining temperature and salinity to stay close to their a priori estimates, but, in practice, constraining density gradients is more efficient. There can still be some problems because the transports can vanish locally even if there is a non-zero vertical shear. There are also some regions such as the deep Eastern Atlantic where the $a$ priori estimates of the density gradients are almost zero. However, since these problems occur in a few boxes only, it is relatively easy to fix them on a case by case basis, for example by putting more weight on the a priori estimate of the local transports. In the example described above, for instance, we would replace the a priori guess $\mathrm{U}_{\mathrm{W}}=1 \pm 5$ by $\mathrm{U}_{\mathrm{W}}=1 \pm 0.5$. One has to be careful not to constrain too tightly an a priori guess which might be wrong, and it is usually necessary to try out different error bars. This can be done because the model can be run very quickly and problems arise in only a few places.

Because the density field is unknown during the Last Ice Age, it cannot be used to prevent the circulation from becoming too sluggish. Another approach to prevent a no-flow solution is to explicitly include the ${ }^{14} \mathrm{C}$ "clock" in the model, but there are not enough data points yet to justify the addition of the $\Delta^{14} \mathrm{C}$ tracer to the model.

The practical approach used in the LGM simulations is to treat the circulation as a parameter. For instance, we take the modern ocean circulation as an a priori estimate of the flow, and try to modify it as little as possible in order to be consistent with glacial tracers. As long as the a priori estimate of the transports is reasonable, it is not necessary to modify it much to make it consistent with the paleo-data. With this approach, the $a$ priori transports are tightly constrained, and the $a$ posteriori estimated transports are unlikely to vanish.

\section{b/ Transports in the Deep Western Boundary Current}

The objective mapping procedure used in the Fukumori et al. atlas tends to smooth the gradients of density, especially in the Deep Western Boundary Current (see discussion in section IV-4). This smoothed density field causes the model to produce unrealistically small transports in the Western Atlantic. Moreover, imposing larger gradients of density does not suffice to increase 
the transports because the model tends to place the level of no motion close to the bottom of the ocean, which results in small southward transports in the deep levels.

Thus, we help the model to get the "right" transports in the modern ocean simulation by constraining more tightly the Deep Western Boundary Current to advect about $15 \mathrm{~Sv}$. This is roughly what one expects from hydrographic and current meter data (Warren, 1981).

The $a$ priori estimate of the density field is obtained from Fukumori et al. (1991); the sampling procedure described in section IV-4 is used for the DWBC data. A reasonable modern ocean solution, satisfying both the physical constraints and the data, can be obtained this way (section V-A).

Note that the a posteriori gradients of density look reasonable and appear to be slightly steeper than the ones we chose a priori which confirms the presumption that the atlas fields are too smooth (Figure IV-14).

\section{c/ Local minima of the cost function}

There is no guarantee that the optimization procedure will find the global minimum of the cost function and indeed it seems to get stuck in local minima more often than it succeeds in avoiding them. Starting the optimization at the a priori estimate of the solution appears logical, and usually gives the best results.

Other starting points are possible. One of them is such that all the variables are initialized at a value of 1 . In this case, the optimization procedure finds a local minimum a hundred times larger than the one obtained when starting at the $a$ priori estimate of the solution. The resulting $a$ posteriori estimate of the solution is inconsistent with the physical constraints (residuals too large). Therefore, local minima can significantly reduce the performance of the model, and picking an appropriate starting point for the optimization is important.

We improved the results of the optimization (further reduced the cost function, and obtained smaller equation residuals) by imposing a net northward flow through $10^{\circ} \mathrm{N}$ below $4 \mathrm{~km}$ depth. Thus, the more information we give to the model, the better it fares. Indeed, the linearizations made at each iteration are more accurate, and the optimization is less likely to get stuck in a local minimum if the range over which the state vector can vary is smaller. 

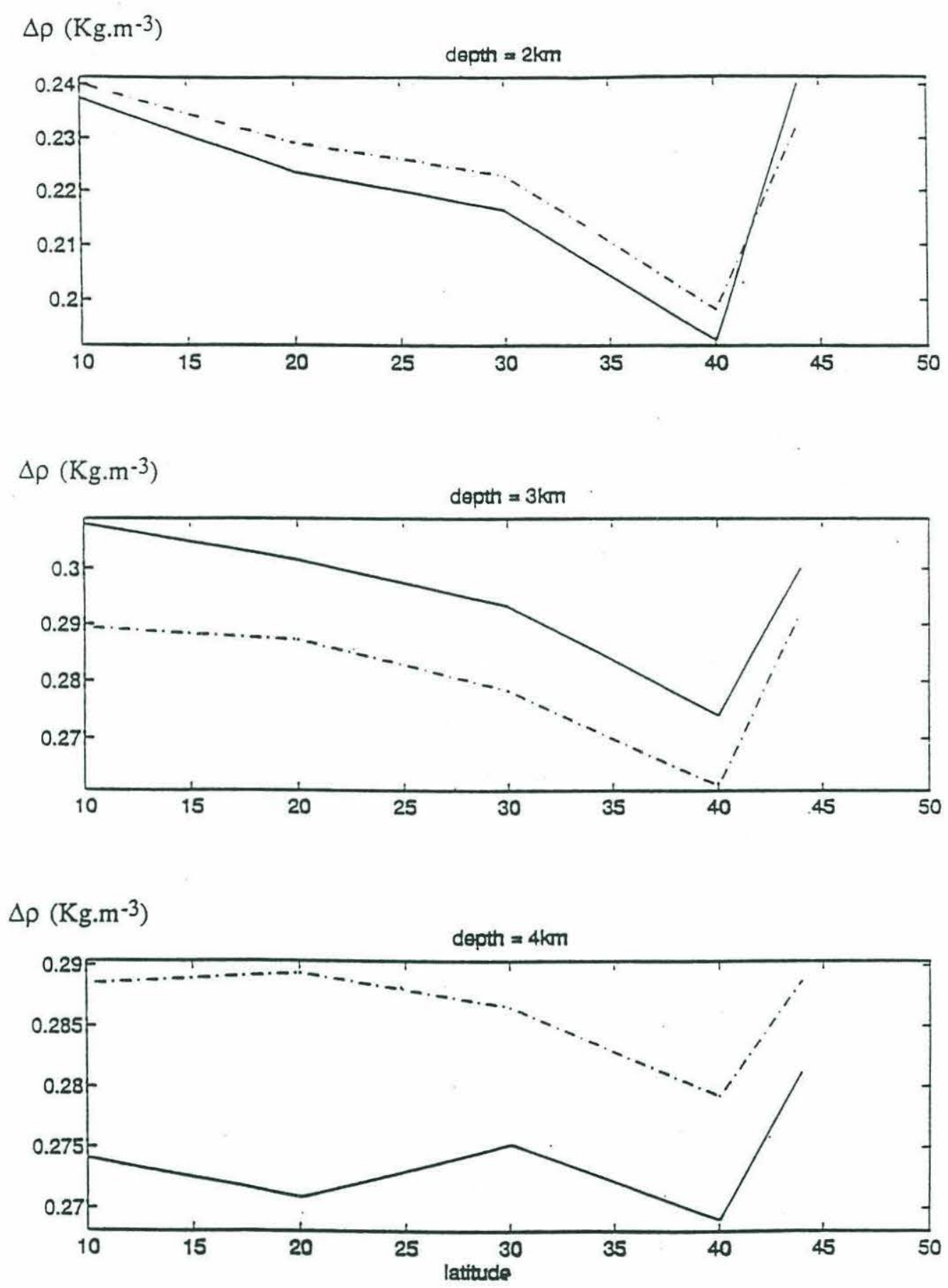

Figure IV-14: A priori density variation (dashed line) and a posteriori density variation (full line) estimated by the model for the modern ocean run described in section V-A, at $60^{\circ} \mathrm{W}, 2 \mathrm{~km}, 3 \mathrm{~km}$, and $4 \mathrm{~km}$ depths (mean density over line subtracted). The a priori density field is obtained by subsampling the tracer distributions given in the Fukumori et al. atlas (1991). Note that the absolute density does not appear in the model equations and the offsets between dashed and full lines are not meaningfull. 
Note that local minima do not seem to be such a problem in other non-linear inverse models (e.g. Mercier et al., 1993). The difficulties we have encountered may be linked to the fact that, unlike most models, the model we use treats temperature and salinity as variables, which results in non-linear tracer conservation equations. The imbalances of these conservation equations are difficult to minimize because of their nonlinearity.

\section{d/ Sensitivity to weight matrices}

Weight matrices are used to insure that the more reliable constraints are better satisfied. The matrix $\mathbf{C}_{\mathbf{o}}$ controls the data misfit and, in theory, should be the covariance matrix of the a priori estimate of the solution. The statistics of the a priori estimate of the solution are not always known, as in the case of the transport variables for instance, but a rough guess is always possible. For example, the flux of water exchanged between two adjacent boxes is typically of the order of $1 \mathrm{~Sv}$ and very rarely above $5 \mathrm{~Sv}$. Thus a sensible guess at the standard deviation for the water transport variables is about 2 or $3 \mathrm{~Sv}$. The diagonal terms of $\mathbf{C}_{\mathbf{0}}$ are taken to be the square of the standard deviation and, in the absence of more information, the non-diagonal terms are assumed to be zero.

It is more difficult to come up with an estimate of the weight matrices $\mathbf{C}_{\mathbf{a}}$ and $\mathbf{C}_{\mathbf{f}}$ associated with the linear and non-linear constraints. One idea is to scale the different equations: for potential temperature for instance, the conservation equation is of the form $\mathrm{U}_{\mathrm{i}} \mathrm{C}_{\mathrm{i}}-\mathrm{U}_{0} \mathrm{C}_{0} \approx 0$ (i for in, ofor out). $\mathrm{U}_{\mathrm{i}}$ and $\mathrm{U}_{0}$ are typically of order $1 \mathrm{~Sv}$ and $\mathrm{U}_{\mathrm{i}}=\mathrm{U}_{0}$ since mass is conserved. $\mathrm{C}_{\mathrm{i}}-\mathrm{C}_{0}$ at a depth of $3 \mathrm{~km}$ is about $0.05^{\circ} \mathrm{C}$. Thus, the imbalance before optimization can be expected to be about $1 \mathrm{~Sv} \times 0.05^{\circ} \mathrm{C}=5 \times 10^{-2} \mathrm{~Sv} .{ }^{\circ} \mathrm{C}$. The objective is to reduce this imbalance by an order of magnitude. We impose a weight $\mathrm{W}$ such that its contribution to the objective function is about one after optimization:

$$
\left[\mathrm{W} \times\left(\mathrm{U}_{\mathrm{i}} \mathrm{C}_{\mathrm{i}}-\mathrm{U}_{\mathrm{o}} \mathrm{C}_{\mathrm{o}}\right)\right]_{\text {after optim. }}^{2}=\left[\mathrm{W} \times \frac{1}{10} \times 5 \times 10^{-2}\right]^{2}=1
$$

which gives $\mathrm{W}=2 \times 10^{-4}$. Of course, we need to scale all the equations in the same manner so that their contribution to the cost function is also one. Each data contribution should also scale like one 
since its contribution to the cost function is $\left(x-x_{0}\right)^{2} / \sigma_{0}^{2}$, where $\sigma_{0}$ is the standard error associated with $\mathrm{x}_{0}$, and we expect $\mathrm{x}-\mathrm{x}_{0}$ to be of order $\sigma_{0}$. Note that if there is a physical constraint which we believe to be less accurate, we can associate it to a smaller weight so that its imbalance is less reduced.

In practice, scaling the equations does not work because, during the minimization of the cost function, the $\left(\mathrm{x}-\mathrm{x}_{0}\right)$ terms remain much smaller than $\sigma_{0}$, and the physical constraint imbalances remain much larger than what they ought to be, especially for the non-linear equations. It is not clear why this approach fails, but it must have something to do with the difficulty involved in minimizing non-linear constraints. This difficulty is clearly illustrated by a graph showing the evolution of the different components of the objective function with the number of iterations (Figure V-A-1). The data misfit as well as the linear constraint contribution almost reach their final values after one iteration (note that the data misfit starts at zero because, in this example, the starting point is the a priori estimate of the solution). On the other hand, the non-linear constraint contribution to the objective function decreases much more slowly, and thus seems to be more difficult to minimize.

In order to achieve a satisfactory minimization, we increase the weights corresponding to the non-linear constraints in the objective function. This, in turn, causes the linear equation residuals to go up, so their weights must be increased as well to keep the residuals to low values (the residuals are deemed acceptable if the maximum residuals are smaller than $10 \%$, and the mean residuals are smaller than $1 \%$ ). Several trials are necessary to find a set of weights such that all the constraints are reasonably satisfied. For the modern ocean inversion, the resulting physical constraint weights are about 100 times larger than the weights put on the a priori estimate of the solution:

$$
\mathbf{A}^{\mathrm{T}} \mathbf{C}_{\mathbf{A}}^{-1} \mathbf{A}+\mathbf{F}_{\mathbf{k}}^{\mathrm{T}} \mathbf{C}_{\mathbf{f}}^{-1} \mathbf{F}_{\mathbf{k}}=\mathrm{O}\left(100 \times \mathbf{C}_{0}^{-1}\right)
$$

see Chapter III for the notation. There is no apparent reason why it has to be $\mathrm{O}(100)$ and not something else. The results depend a lot on the different weight matrices, and the lack of a rigorous way to estimate the weights appears to be a major difficulty with least square methods. Even relatively small changes in the weights can make significant improvements in the results, particularly for the non-linear constraints. The optimization tends to end up in groups of solutions. 
By modifying a weight, one can jump from one group of solutions to another. The explanation is probably that by modifying weights, one modifies the shape of the objective function and creates or removes local minima or bifurcations. Since we cannot find a rigorous way to estimate the weight matrices, we have to use a trial and error approach.

For LGM runs, it is usually sufficient to set the matrix $\mathbf{C}_{\mathbf{f}}$ to zero, and simply adjust the coefficients in the matrix $\mathbf{C}_{\mathbf{A}}$. The solution does not depend strongly on the choice of the weighting coefficients associated with the linear constraints, and a rough estimate of the weight matrix $\mathbf{C}_{\mathbf{A}}$ is sufficient. Setting $\mathbf{C}_{\mathbf{f}}$ to zero results in very small imbalances in the non-linear equations, thereby eliminating the possibility of having artificial source terms in the tracer conservation equations. This approach is not rigorous because the conservation equations in the model are only a numerical approximation of what happens in the real ocean, and should not be strictly enforced. The main difficulty associated with this approach is that solutions might be missed because they are not fully consistent with the model's conservation equations, even though they are otherwise acceptable. In practice, this difficulty is mitigated by the fact that the optimization procedure is stopped after a limited number of iterations, before a perfect fit to the model tracer conservation equations is reached. Also, round-off errors in the numerical calculations prevent a such a perfect fit.

In the next chapter, we see that the non-linear inverse procedure can be successfully applied to real ocean problems. Several difficulties have to be overcome such as preventing a no flow solution, obtaining realistic transports in the DWBC, avoiding local minima and evaluating weight matrices. We use ad hoc procedures to solve these problems, and there is still some room for improvement. The solutions obtained may not be optimal. More importantly, there may exist other solutions at least as satisfactory as the ones we find. 


\section{Chapter V - Changes in the Deep Atlantic circulation during the Last Glacial Maximum. Results from a quantitative inverse modeling study.}

We saw in chapter II that the LGM water mass distribution was different from its modern counterpart. The extent of these differences cannot be quantified yet, because of the lack of information on the end-member compositions. It seems clear, however, that the volume of Lower NADW was reduced, and that $\mathrm{AABW}$ extended farther north during the LGM. This smaller volume of Lower NADW has been attributed to a reduction in the rate of formation of lower NADW (see chapter II).

This chapter tries to demonstrate, however, that the rate of formation and the volume of a deep water mass are to some extent independent from each other. This independence is evident in at least one extreme, but acceptable case if the only data to constrain the ocean circulation are passive tracer data. In case of no circulation at all, and no biological activity, there can still be water masses which simply stay where they are. Therefore, the volume of a water mass is not a direct indication of the rate of formation of this water mass.

Moreover, it is not clear whether a significant redistribution of the water masses requires significant circulation changes. It seems intuitively plausible that a small reduction in the production of NADW, sustained over a long period of time, could result in a large reduction of the volume of NADW. Thus, while changes in the North Atlantic water mass composition and, to some extent distribution, are well established, it is not clear how much they are related to circulation changes.

To explore the range of possible circulations, we use the coarse resolution inverse model of the Deep Atlantic described in the previous chapter. The geochemical tracers $\delta^{13} \mathrm{C}$ and $\delta^{18} \mathrm{O}$ are used as constraints on the circulation during the Last Glacial Maximum. Note that our purpose is not to explain how the tracer field went from its LGM to its modern distribution (a time-dependent model would be necessary to do so), but to determine the range of circulations consistent with a snapshot of the $\delta^{13} \mathrm{C}$ and $\delta^{18} \mathrm{O}$ data during the Last Ice Age. 
In part $\mathrm{A}$, we analyze the results of a modern ocean simulation which will serve as a reference run to which paleo-simulations will be compared. Part B describes the results of 2 LGM simulations. The first simulation finds the smallest circulation changes necessary to fit the LGM North Atlantic data. The second one simulates a reduced Lower NADW rate of formation scenario. These simulations are then extended to the South Atlantic using a simple kinematic boxmodel. Part $\mathrm{C}$ analyzes how and why different circulations are consistent with the paleo-data.

\section{A - Run [0]: A reference solution in the modern ocean}

The objectives of this simulation are to test the model using real data and to obtain a reference run to which the last glacial simulations can be compared.

The circulation in the Deep North Atlantic (for depths greater than $1 \mathrm{~km}$ ) is estimated by combining the model physics with potential temperature, salinity and phosphate data, subsampled every $10^{\circ}$ from the Fukumori et al. atlas. As shown in Figure V-A-1, the objective function is reduced from $3 \times 10^{9}$ down to about $10^{3}$. The optimization is stopped after 10 iterations, since no further progress is made. The $a$ posteriori equation residuals are less than about $10 \%$ ( Figure VA-2; see section IV-5 for the exact definition of the residuals) which means that the physical constraints are satisfied at least to the first order. Mass is essentially exactly conserved with residuals smaller than $0.01 \%$. There is a very good improvement over the a priori estimate of the solution which did not satisfy the equations at all. The mean residuals are at around $1 \%$, so on average the equations are satisfied up to the second order.

The imbalances integrated over the whole model domain represent an artificial source of mass and tracer. However, these artificial sources are very small. Tracer imbalances integrated over the whole model domain would take more than 100000 years to change the basin mean potential temperature by $1^{\circ} \mathrm{C}, 8$ million years to change the mean salinity by $1 \mathrm{PSS}$, and 2 million years to change the mean phosphate concentration by $1 \mu \mathrm{mol} / \mathrm{kg}$. Thus, there are small sources and sinks of tracers in each box but they nearly cancel each other. The subgrid scale phenomena randomly perturb the large scale balances but they do not play a significant role overall, and the coarse resolution model seems to capture the fundamental aspects of the ocean circulation. 


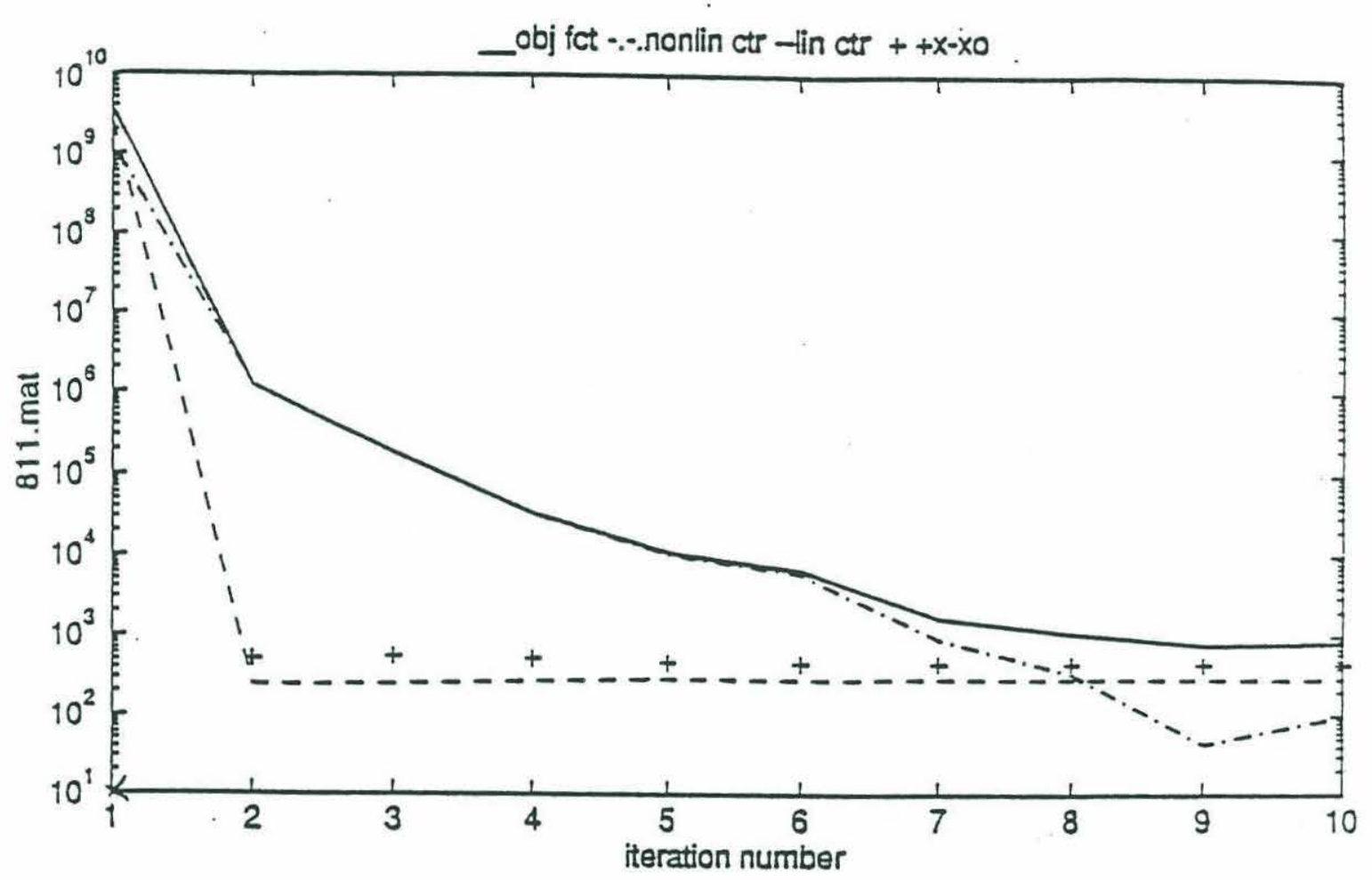

Figure V-A-1: Objective function versus number of iteraions, for the modern ocean simulation. Dot-dash line represents the contribution of the nonlinear physical constraints to the objective function: $\mathbf{f}(\mathbf{x})^{\mathrm{T}} \mathrm{C}^{-1} \mathbf{f}(\mathbf{x})$. Dashed line represents the contribution of the linear physical constraints to the objective function: $(\mathbf{A x}-\mathbf{b})^{\mathrm{T}} \mathrm{C}_{\mathbf{A}}{ }^{-1}(\mathbf{A x}-\mathbf{b})$. Pluses represent the data misfit term $\left(x-x_{0}\right)^{\mathrm{T}} \mathrm{C}_{0}{ }^{-1}\left(\mathbf{x}-\mathrm{x}_{0}\right)$. The initial data misfit is zero because the starting point of the minimization is the a priori solution, $\mathbf{x}_{\mathbf{0}}$. Full line represents the objective function, which is the sum of the three preceding terms. 

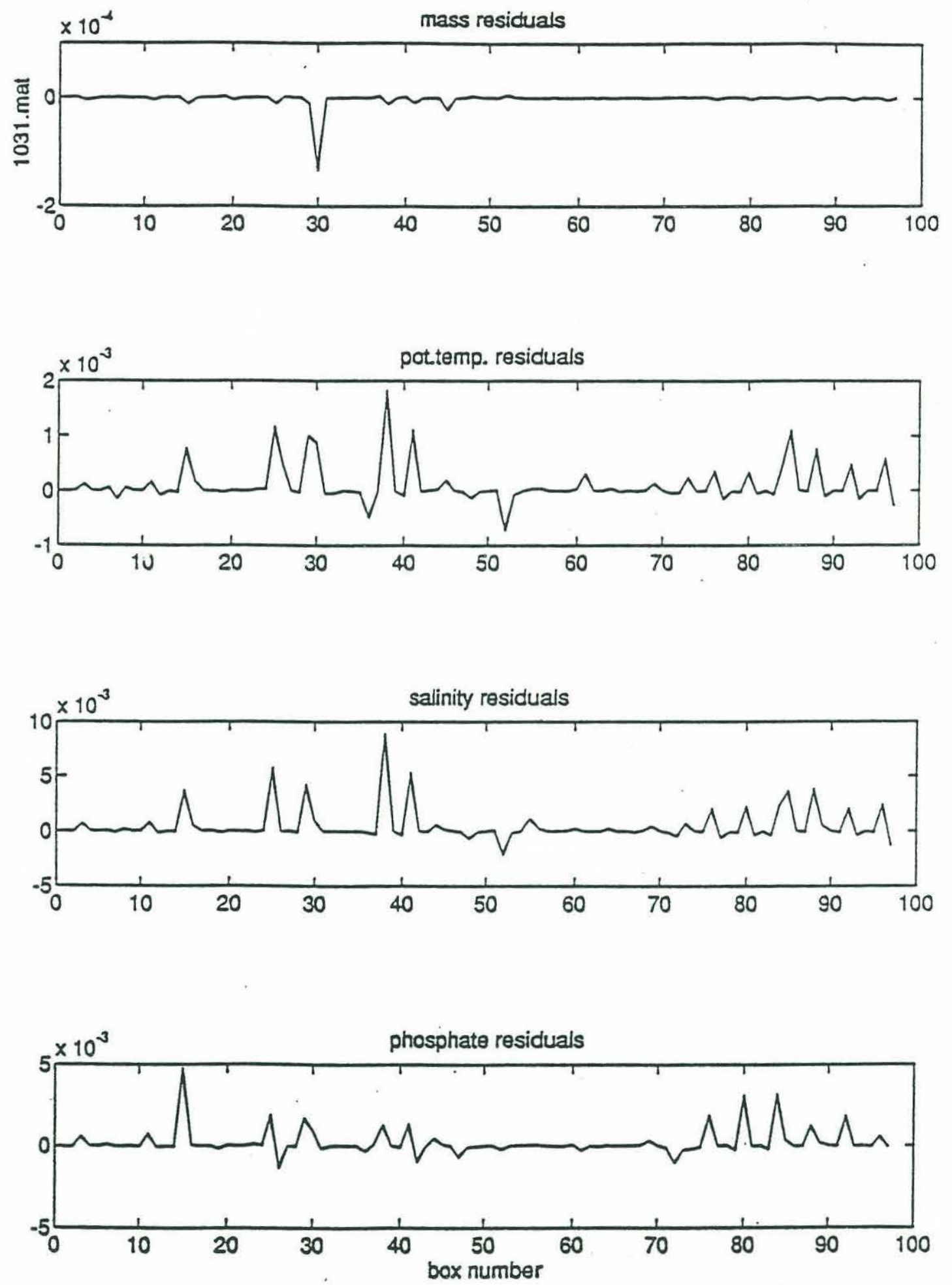

Figure V-A-2-a: Residuals after minimization in each volume element of the model, for the modern ocean simulation. There are 97 such elements (boxes). The residuals are defined as the sum of the fluxes entering the box divided by the absolute value of the largest flux leaving or entering the box. 

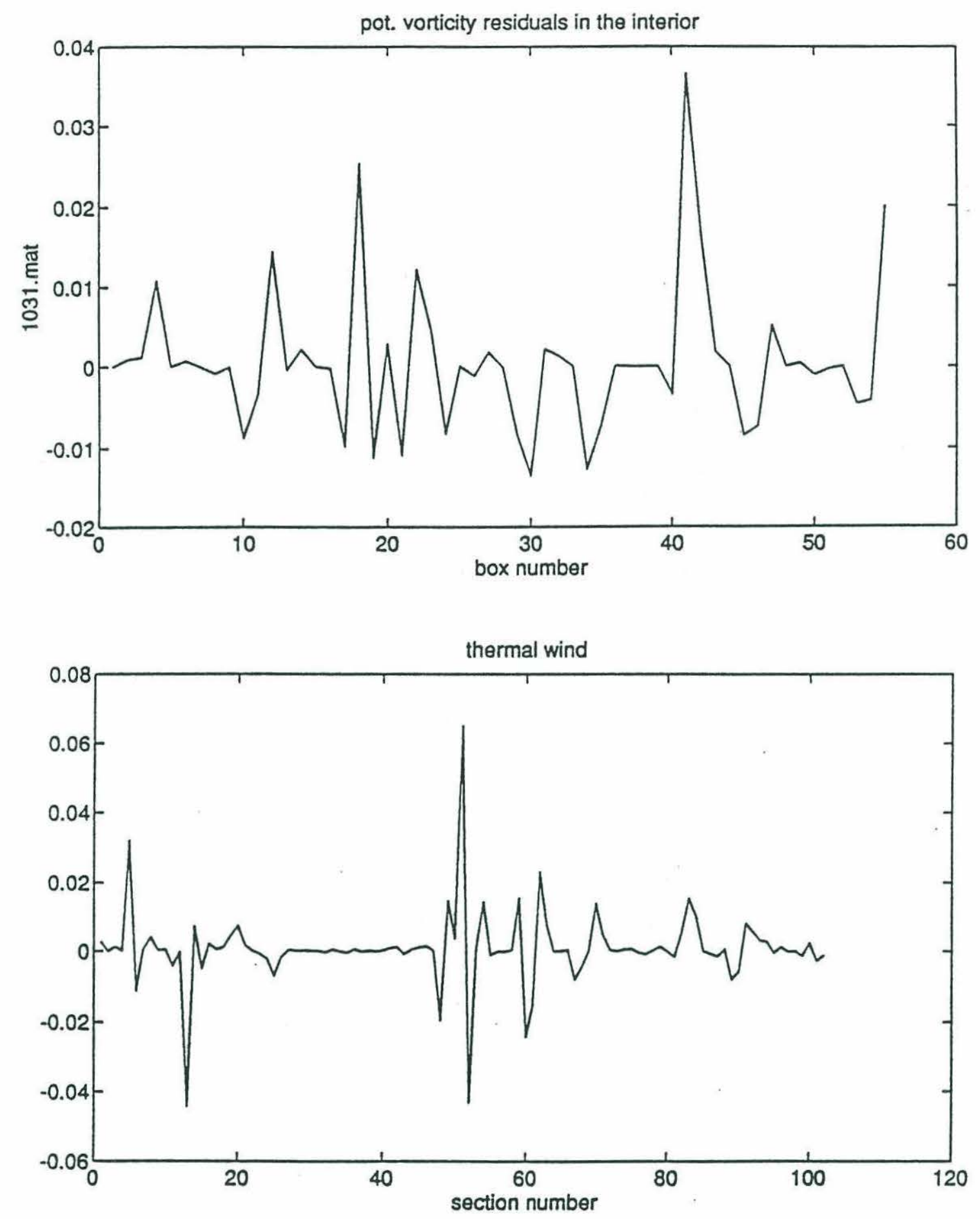

Figure V-A-2-b: Upper panel: Residuals after minimization of the linear vorticity balance in each box where it is imposed. Lower panel: Residuals after minimization of the thermal wind balance for each section where it is imposed. Modern ocean simulation. The residuals are defined as the left hand side minus the right hand side of the equations, divided by the largest of those two terms. 
Potential temperature, salinity, and phosphate data are modified by the inversion as shown in Figures V-A-3\&4 $\left(20^{\circ} \mathrm{W}\right.$ and $\left.50^{\circ} \mathrm{W}\right)$. One can get an idea of the distribution of $\delta^{13} \mathrm{C}$ by assuming a linear relationship between phosphate and $\delta^{13} \mathrm{C}$ :

$$
\delta^{13} \mathrm{C}=-0.93 \times \mathrm{PO}_{4}+2.2
$$

(the slope of this relation, -0.93 , is taken from Broecker and Peng, 1982, page 309). The $\delta^{13} \mathrm{C}$ values diagnosed from the model phosphate distribution appear to agree reasonably well with the GEOSECS data (replace the phosphate isolines in Figures V-A-3\&4 by the appropriate $\delta^{13} \mathrm{C}$ values, and compare with Kroopnick, 1985). Deep Water can be clearly seen in the salinity profile at $50^{\circ} \mathrm{W}$ as the water bounded by the upper and lower 34.9 PSS isolines (note that the contours look a bit distorted because of the very low resolution used in the model). The a posteriori isolines do not significantly depart from the a priori ones. The main difference in the phosphate sections is that the $1.2 \mu$ mol. $\mathrm{kg}^{-1}$ isoline has been shifted upward in the a posteriori estimate at $50^{\circ} \mathrm{W}$. However, the a posteriori profiles are plausible, and the graphic differences may be partly due to different contouring of the tracer concentrations. Indeed, after inversion, the phosphate concentrations as well as potential temperature and salinity values are consistent with their $a$ priori values within the error bars. A histogram of the data misfit (Figure V-A-5) shows that most of the a posteriori tracer concentrations are less than one standard deviation away from the $a$ priori estimates. Less than $1 \%$ of the data are more than 3 standard deviations from their initial values, which is what we expect for a normal distribution. However, the histogram is a bit too flat to be truly normal. The long tailed density functions may be due to the presence of a few blunders in the data set. The presence of blunders would not be surprising since some data have to be extrapolated at grid points which correspond to land locations in the real world (see section IV-4), and some of the extrapolated values may be inconsistent with the rest of the data.

The circulation estimated by the model is shown in Figure V-A-6. The deep ocean circulation is not precisely known, even in the Deep North Atlantic which has been more intensively explored than the other basins, so the details of the solution cannot be verified. However, the inferred circulation seems reasonable. The upper layer, between 1 and $2 \mathrm{~km}$ depths, exhibits only weak transports in the DWBC boxes. These weak transports are probably an artefact of the Fukumori et 

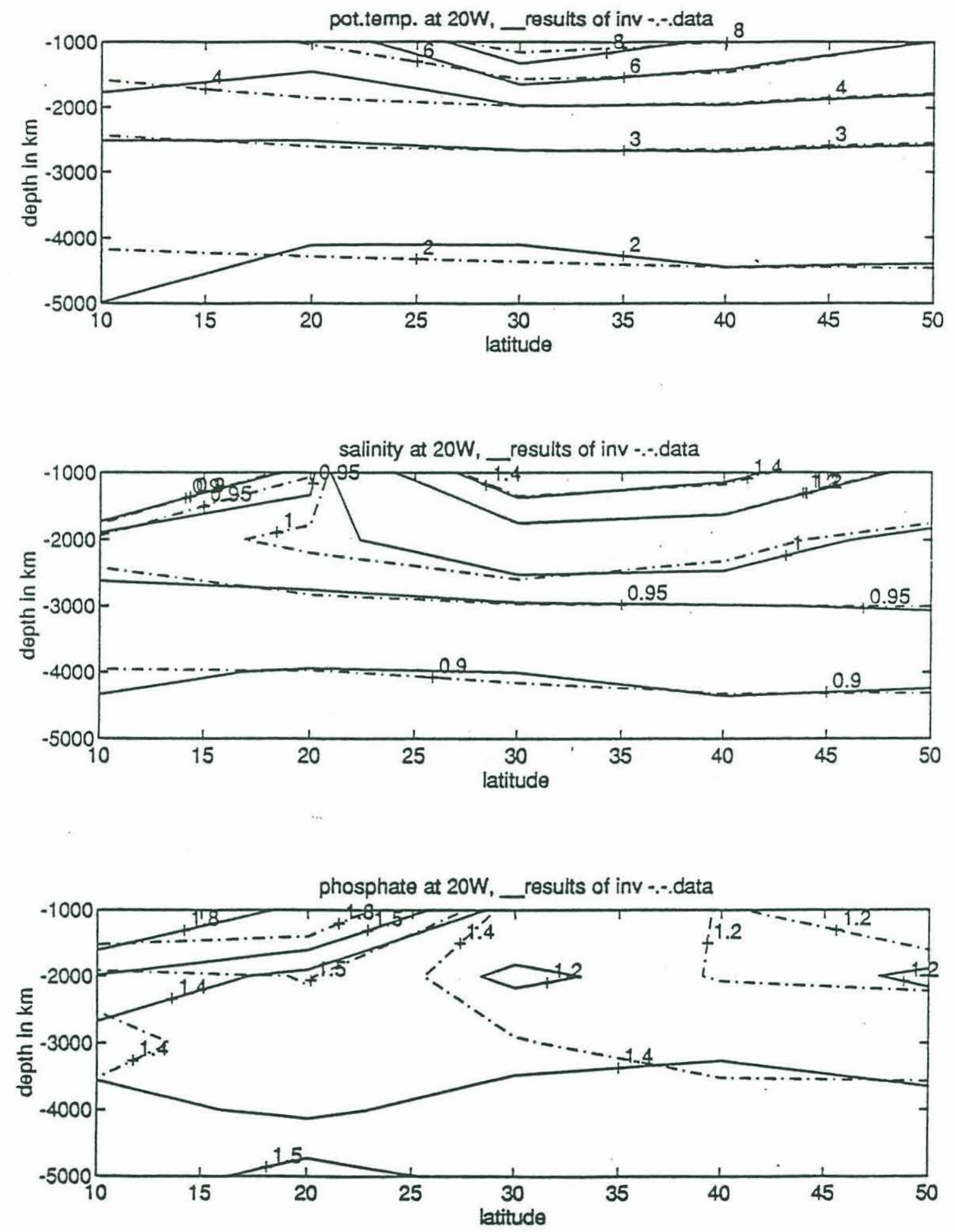

Figure V-A-3: Meridional sections of potential temperature $\left({ }^{\circ} \mathrm{C}\right)$, salinity (PSS -34$)$, and phosphate $(\mu \mathrm{mol} / \mathrm{l})$ in the modern ocean, at $20^{\circ} \mathrm{W}$. Full lines represent model predictions. Dot-dash lines represent tracer distributions in the Fukumori et al. atlas, subsampled at each model grid-point. 

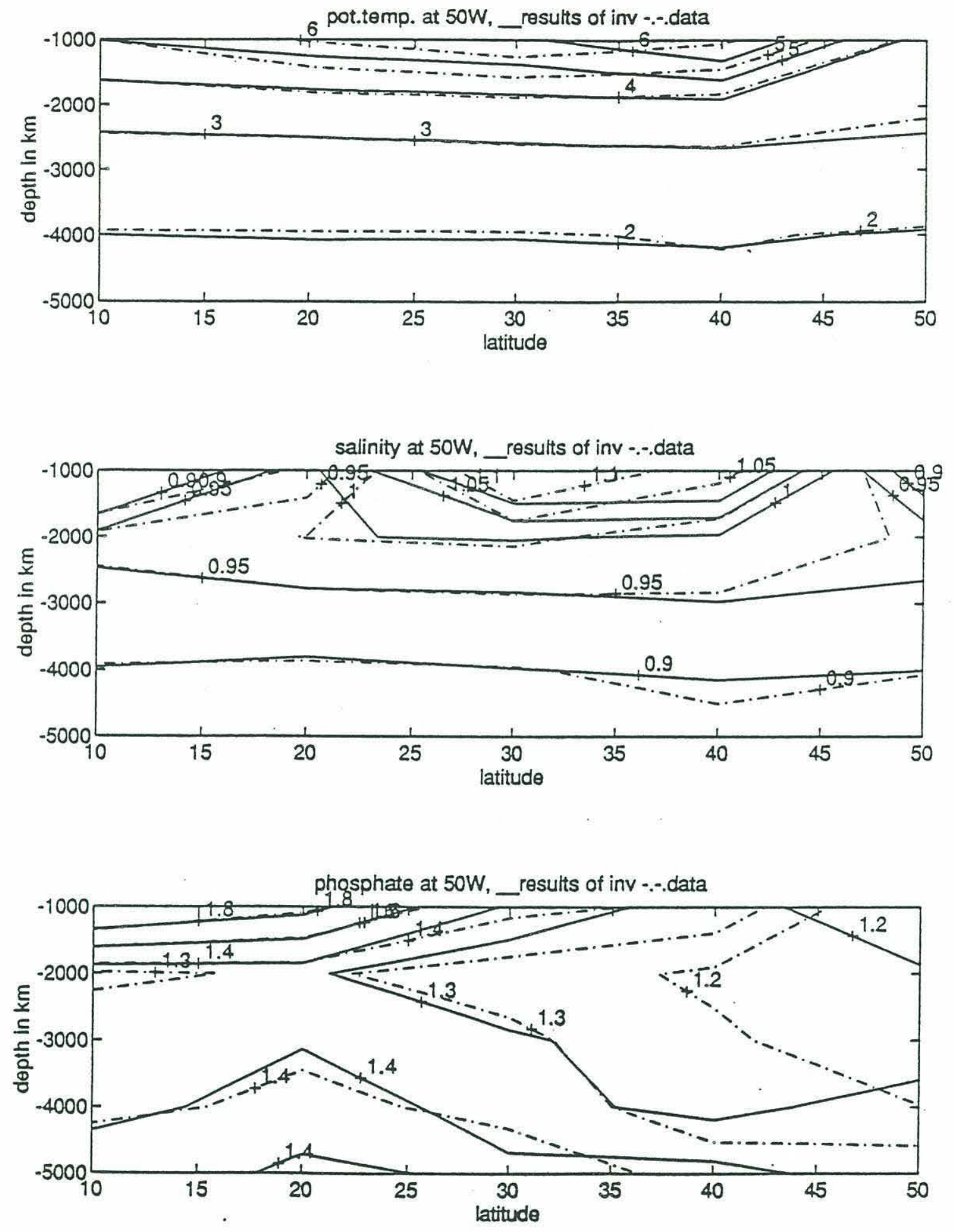

Figure V-A-4: Meridional sections of potential temperature $\left({ }^{\circ} \mathrm{C}\right)$, salinity (PSS -34$)$, and phosphate $(\mu \mathrm{mol} / \mathrm{l})$ in the modern ocean, as in Figure $\mathrm{V}-\mathrm{A}-3$ except for $50^{\circ} \mathrm{W}$. Full lines represent model predictions. Dot-dash lines represent tracer distributions in the Fukumori et al. atlas, subsampled at each model grid-point. 

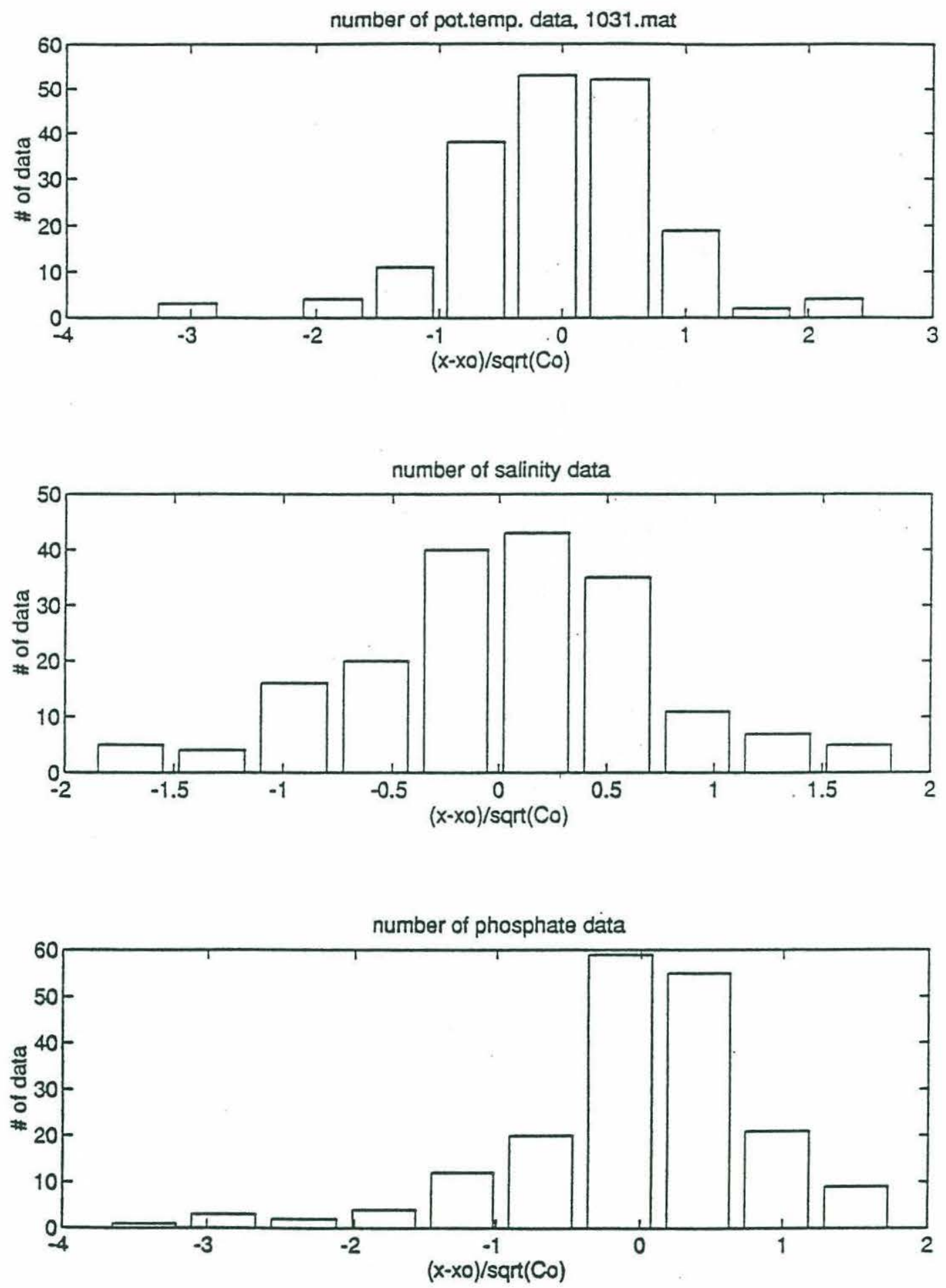

Figure V-A-5: Histogram of the data misfits after minimization for potential temperature, salinity, and phosphate. Modern ocean simulation. Data misfit defined as tracer value predicted by the model minus a priori tracer value. Abscissa: data misfit divided by the standard deviation of the uncertainty in the a priori estimate. Ordinate: number of data points in each interval of the histogram. 

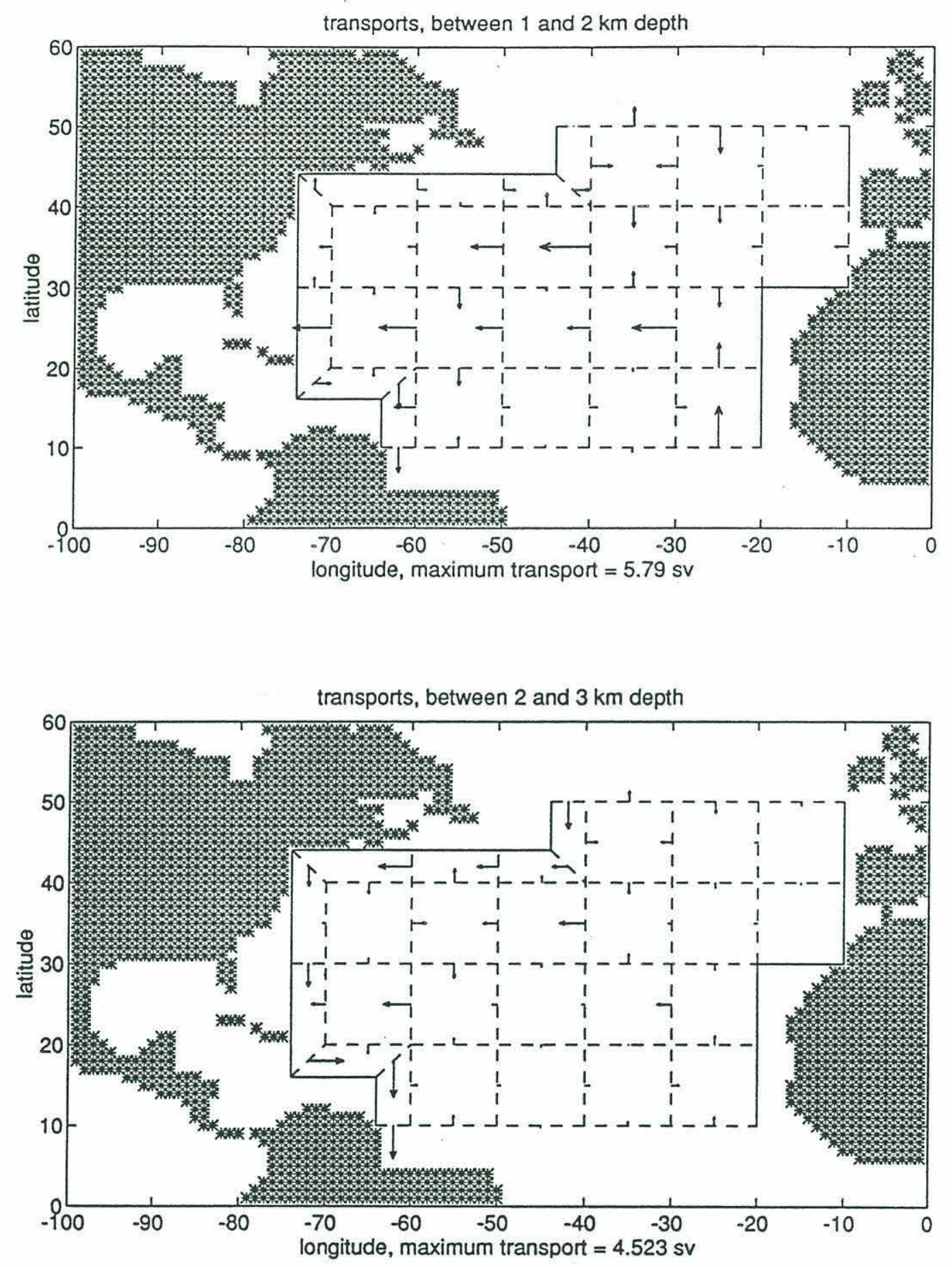

Figure V-A-6-a: Horizontal transports (in Sv) in the upper layers of the model, in the modern ocean simulation. The transports associated with the longest arrow is indicated below each panel. The vectors in the westernmost boxes represent the transports in the DWBC. 

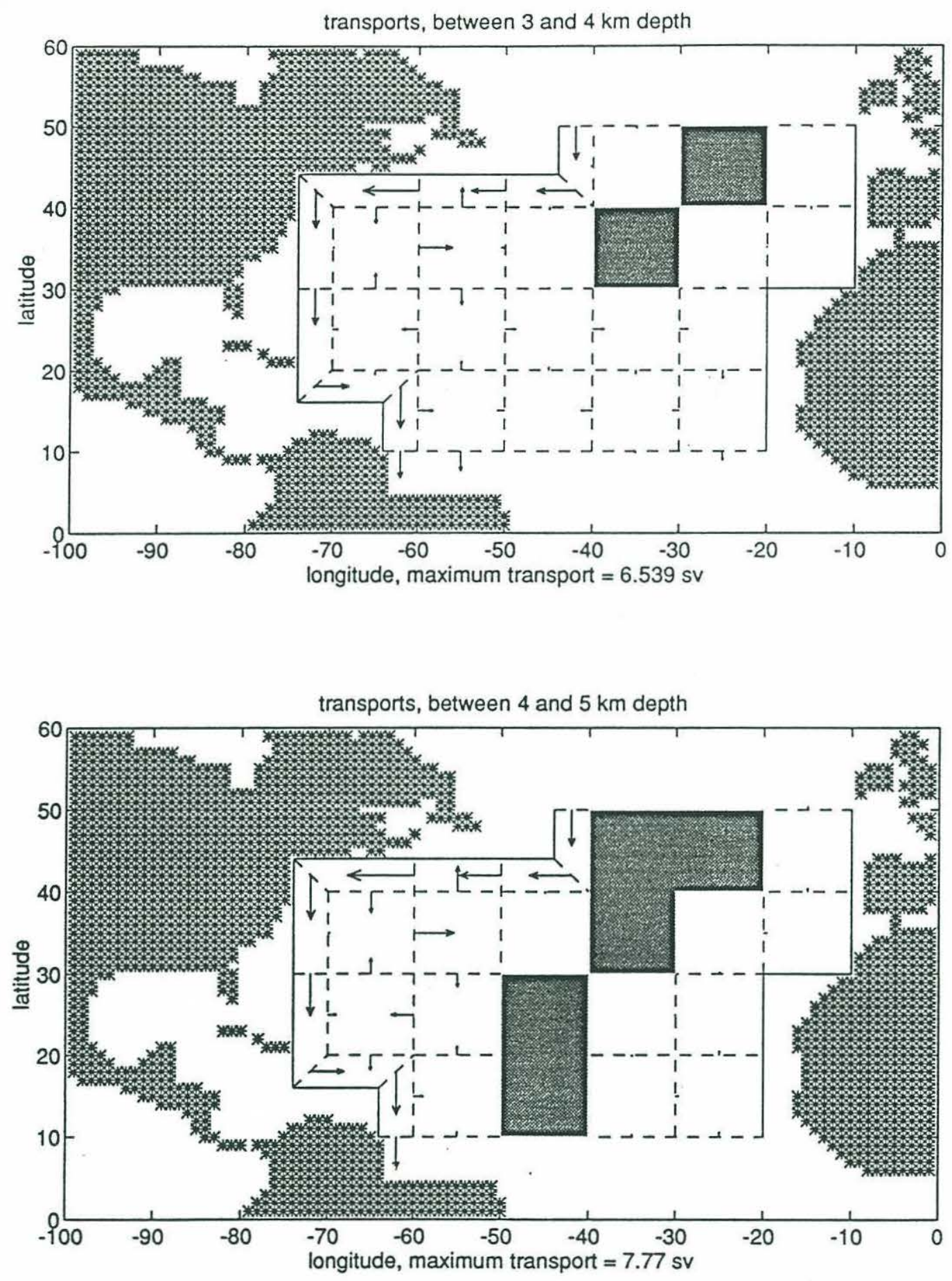

Figure V-A-6-b: Horizontal transports (in Sv) in the lower layers of the model, in the modern ocean simulation. The transports associated with the longest arrow is indicated below each panel. The vectors in the westernmost boxes represent the transports in the DWBC. 
al. atlas fields. Indeed, the inverse model of Martel and Wunsch (1993), which uses the same atlas fields as the present model, also produces weak transports at $2000 \mathrm{db}$, despite a much higher resolution of their finite difference grid. Also, the circulation at $2000 \mathrm{db}$ in their model is qualitatively consistent with the transports between $1 \mathrm{~km}$ and $2 \mathrm{~km}$ depths in the interior of the ocean in the present model.

The Deep Western Boundary Current carries about $15 \mathrm{~Sv}$, divided into roughly equal transports of $5 \mathrm{~Sv}$ each in the 3 deepest layers of the model. The fact that we get reasonable transports for the Deep Western Boundary Current is not too surprising since we "helped" the model to find such a solution by putting large weights on the a priori estimate of the DWBC transports (see section IV-6-b). The zonally integrated transport is about $16 \mathrm{~Sv}$ flowing southward at $30^{\circ} \mathrm{N}$ which is consistent with Hall and Bryden (1982; they find a transport of $18 \mathrm{~Sv}$ between 1 $\mathrm{km}$ depth and the bottom). 1.6 Sv flows out of the Mediterranean between $1 \mathrm{~km}$ and $2 \mathrm{~km}$ depth. This number is slightly different from Ochoa and Bray's (1991) estimate of $2.1 \pm 0.3 \mathrm{~Sv}$ (1 Sv of outflow $+1 \mathrm{~Sv}$ of entrainment) but is acceptable in view of the very simplistic representation of the topography and of the coast in the model. In the model, the water originating from the Mediterranean outflow spreads south-westward probably in order to reproduce the Mediterranean salt tongue, which is the most noticeable feature in the data at intermediate depths (see Fukumori et al., 1991).

The flow across $10^{\circ} \mathrm{N}$ is northward in the deepest layer of the Eastern Atlantic (between $4 \mathrm{~km}$ and $5 \mathrm{~km}$ depth). McCartney et al. (1991) calculated that there are about $2 \mathrm{~Sv}$ going north as an eastern boundary current along the Mid-Atlantic Ridge and 2 Sv going north in the Eastern Atlantic, after having crossed the Vema fracture zone at $10^{\circ} \mathrm{N}$. The model finds $0.65 \mathrm{~Sv}$ going north, west of the Mid-Atlantic Ridge, and 1 Sv going north in the Eastern Atlantic. These numbers differ from McCartney et al.'s but their calculations concern boundary currents whereas the model computes integrated transports. We can only tell that the estimated transports are in the same sense and are of comparable magnitude.

Thus, we have estimated a solution which is consistent with the available hydrographic data and with simplified, yet realistic physics, and which does not look unreasonable. It might not accurately represent the true ocean circulation but at least it is internally consistent. We will use 
this run as a modern ocean reference solution to which last glacial maximum simulations can be compared. The maximum physical constraint residuals could not be reduced below $10 \%$ and their mean below about $1 \%$. So, the Last Glacial Maximum runs will be considered satisfactory if the associated residuals can be brought down to the same level.

\section{B - Last Glacial Maximum Inversions}

We saw in part A how the modern ocean circulation can be quantitatively estimated from tracer data and simplified physics using optimization techniques. The same approach will now be applied to explore the range of solutions consistent with paleoclimate data. Our objective is to show that several different ocean circulations can produce the Last Ice Age tracer distribution.

The first experiment consists of taking the output of the modern ocean simulation as an a priori estimate of the LGM circulation and trying to modify it as little as possible in order to make it consistent with the Last Ice Age $\delta^{18} \mathrm{O}$ and $\delta^{13} \mathrm{C}$ data. The second experiment shows that a circulation with a rate of formation of North Atlantic Deep Water of about half that of the modern ocean is also consistent with the data. These scenarios are then extended to the South Atlantic using a simple kinematic box-model. Before seeing the results of these simulations in detail, some difficulties encountered when using the model to do paleo-climate simulations are reviewed.

\section{1) Difficulties associated with Last Glacial Maximum simulations:}

The problems encountered in the modern ocean simulation (described in section IV-6) also apply to paleo-climate inversions, and are often more difficult to overcome because of the paucity of data.

Because of the large observational uncertainties and the scarcity of the data, the model variables are poorly constrained by the observations, and can vary over a wide range of values. This wider range of values makes the choice of a good starting point for the optimization more difficult in paleoclimate simulations than in the modern ocean. The optimization is more likely to 
find unsatisfactory local minima. Because the starting point is likely to be far from the optimal solution, more iterations are usually necessary to converge to the optimal solution.

Moreover, the optimization procedure is more subject to numerical noise. The minimization of the cost function involves the inversion of the Hessian matrix (matrix of the second partial derivatives of the objective function) at each iteration. In the modem ocean simulation, the inverse of the data covariance matrix, $\mathbf{C}_{0}{ }^{-1}$, eliminates the small singular values associated with the physical constraints (Mercier et al., 1993). In paleo-simulations, large error bars in LGM data translate into small diagonal elements in $\mathrm{C}_{\mathbf{0}}{ }^{-1}$ matrix and thus cause poor numerical conditioning when inverting the Hessian matrix. This poor conditioning can result in a poor rate of convergence, or an optimization which does not converge at all.

The model also tends to produce unrealistic tracer distributions in paleo-climate simulations. Because many grid points are not constrained by any observations, the model is free to adjust the tracer variables there, and create complicated structures in the tracer field that we have no reason to believe. In particular the salinity field, which is not constrained by any data, tends to exhibit incoherent patterns and steep gradients that are unlikely to occur in the real ocean (where we expect some smoothing of the tracer field due to eddies and other mixing effects). Moreover, extrema in tracer concentrations can appear in the middle of the model domain, away from the sources of deep water. These extrema are unphysical but the model numerical scheme for tracer conservation equations does not prevent them, as illustrated in Figure V-B-1 in the context of a simple model of the advection of tracer in a channel. Using a numerical scheme similar to the one used in the model, we can obtain interior extrema in the tracer distribution (Figure V-B-1). 


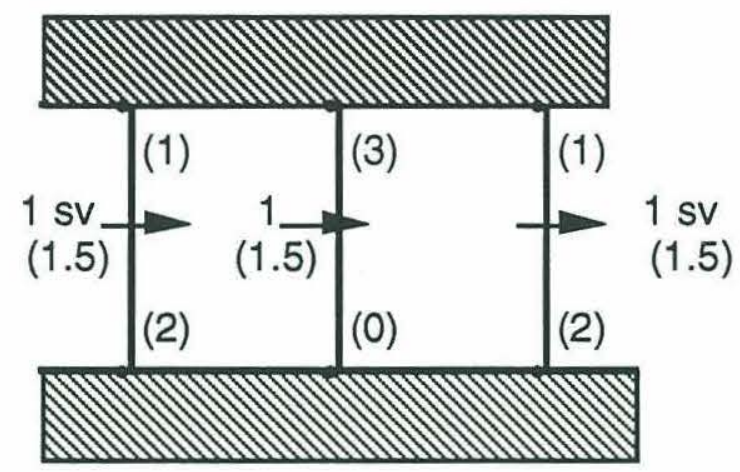

Figure V-B-1: $1 \mathrm{~Sv}$ of water is advected through the channel. The numbers between parenthesis designate tracer concentrations, the other numbers are transports in Sv. The mean tracer concentration over a section of the channel is the average of the upper and lower values since the tracer concentrations are defined at the corners of the box. The solution shown above perfectly conserves mass and tracer but does not prevent extrema in the tracer field such as the concentration of 3 tracer units at the upper middle point.

The solution shown in Figure V-B-1 is consistent with mass and tracer conservation but produces a maximum of tracer concentration away from the sources. Extrema occur in other numerical schemes. For instance, if the concentrations are defined at the center of each interface, there can still be a minimum in the interior of the model domain (see Figure V-B-2).

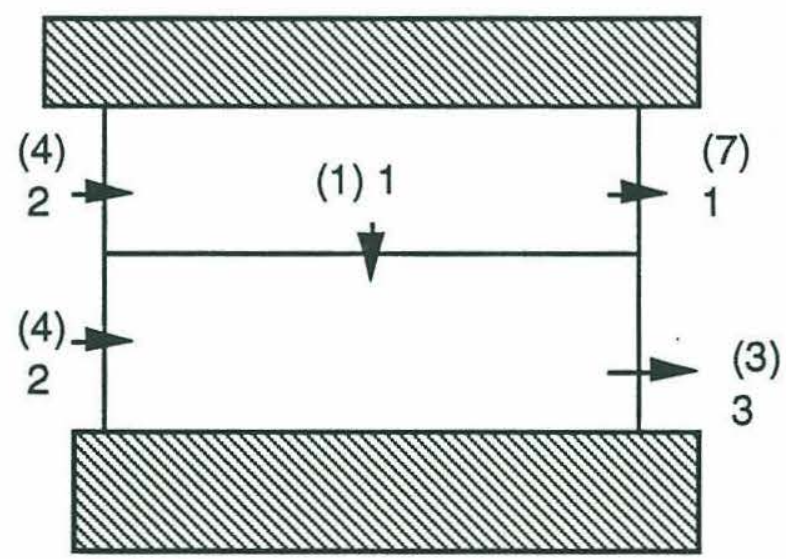

Figure V-B-2: Another example of scheme which can produce extrema of tracer concentration. The tracer concentrations are defined at the center of each side of the boxes. The numbers between parenthesis designate tracer concentrations, the other numbers are transports in Sv. In this example, the interior tracer concentration is lower than any of the boundary values. 
One scheme which prevents tracer extrema is the so-called up-stream scheme. This scheme advects the upstream tracer conservation as shown in Figure V-B-3 for a C-grid (Arakawa and Lamb, 1977).

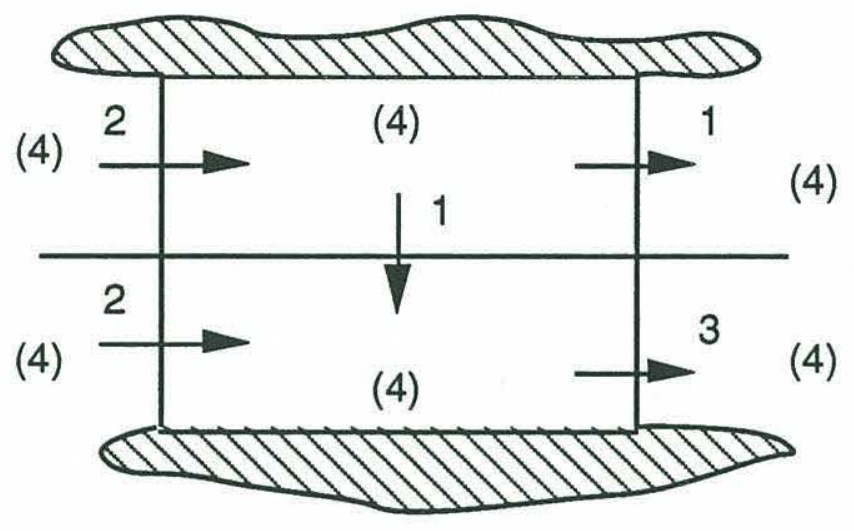

Figure V-B-3: Upstream scheme. In this simple example, the tracer concentration is homogeneous at the source; it cannot change downstream. This scheme always prevents tracer concentration extrema, even in three dimensions. Numbers between parenthesis designate tracer concentrations at the center of each box.

However, the upstream scheme associated with a C-grid is inconvenient for the discretization of the thermal wind balance because the tracer concentrations are not located at the box interfaces. Also, when using this scheme, the physical constraints are continuous but not their derivatives, as shown in the following example:

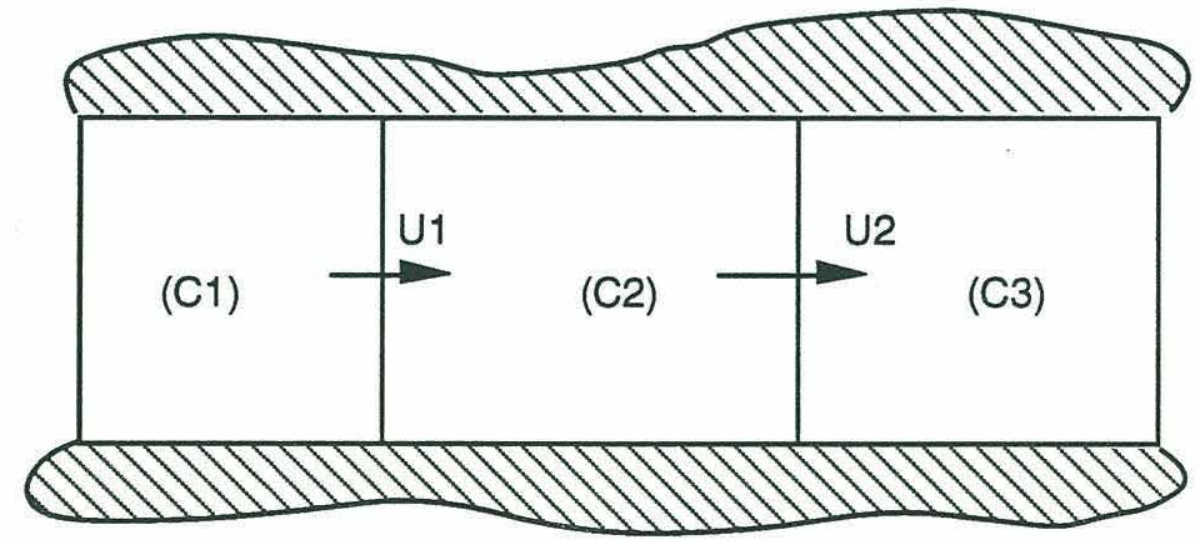

Figure V-B-4: Up-stream scheme discretization of the tracer balance in the middle box of a channel 
The tracer budget for the middle box is:

$$
\begin{aligned}
\mathrm{f} & =\mathrm{U} 1 \times \mathrm{C} 1-\mathrm{U} 2 \times \mathrm{C} 2=0 & & \text { if } \mathrm{U} 1>0 \text { and } \mathrm{U} 2>0 \\
& =\mathrm{U} 1 \times \mathrm{C} 2-\mathrm{U} 2 \times \mathrm{C} 3=0 & & \text { if } \mathrm{U} 1<0 \text { and } \mathrm{U} 2<0
\end{aligned}
$$

and the derivatives of the tracer conservation constraints are of the type:

$$
\begin{aligned}
\mathrm{F}=\partial \mathrm{f} / \partial \mathrm{U} 1 & =\mathrm{C} 1 & & \text { if } \mathrm{U} 1>0 \text { and } \mathrm{U} 2>0 \\
& =\mathrm{C} 2 & & \text { if } \mathrm{U} 1<0 \text { and } \mathrm{U} 2<0
\end{aligned}
$$

Thus F, the derivative, depends on the sign of U1 and is not continuous when U1 goes to zero. The simple optimization procedure used in the model is not sophisticated enough to handle this type of discontinuity. The model variables tend to oscillate between two states, one where U1 is positive, and the other where $\mathrm{U} 1$ is negative, neither of these two states being satisfactory. The discontinuous derivatives essentially give the model contradictory information: at one step it has to go along one descent direction, at the next step along a totally different direction, and then back again to the first direction. This contradictory information prevents the convergence of the optimization procedure. The upstream scheme was abandoned because of this technical difficulty.

One approach to partially removing tracer extrema, is to impose some smoothing on the tracer field. For the example shown in Figure V-B-1 with enough smoothing, very large extrema will disappear and the middle values will be something like $[1.1,1.9]$ instead of $[3,0]$. In the model, smoothing can be imposed by constraining the tracer field to satisfy Laplace's equation, as a weak constraint:

$$
\nabla^{2} \mathrm{C} \approx 0
$$

where $\mathrm{C}$ is the tracer concentration. The numerical discretization of Laplace's equation can easily be done on the model numerical grid (Figure V-B-5).

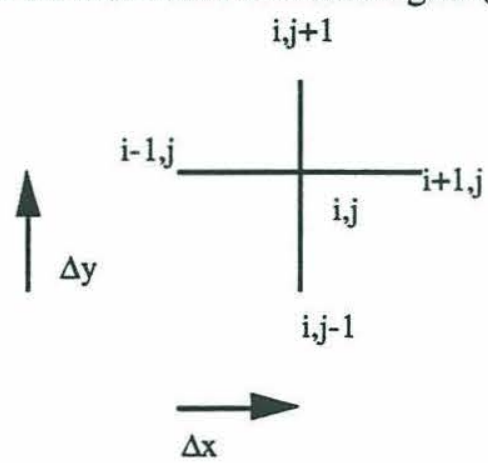

Figure V-B-5: Tracer grid-points in the model 


$$
\nabla^{2} \mathrm{C}=\frac{\mathrm{Ci}+1, \mathrm{j}-2 \mathrm{Ci}, \mathrm{j}+\mathrm{Ci}-1, \mathrm{j}}{\Delta \mathrm{x}^{2}}+\frac{\mathrm{Ci}, \mathrm{j}+1-2 \mathrm{Ci}, \mathrm{j}+\mathrm{Ci,j}-1}{\Delta \mathrm{y}^{2}}
$$

However, smoothing must be large enough to be effective but not so large as to remove all the signal from the data. Moreover, it is an artificial constraint, and there is always the risk that some solutions that would otherwise be consistent, are ignored because they are not as smooth as is specified in the model.

Another approach is to guess what the tracer field should look like in regions where there are not enough observations, and constrain the model to reproduce this guess. We sometimes have a fair idea of what the data should, or could, look like. For instance, there are a lot of $\delta^{13} \mathrm{C}$ data in the LGM Eastern Atlantic so vertical profiles can be reconstructed there. It seems reasonable to extrapolate these vertical profiles to the Western Atlantic, at least above the Mid-Atlantic Ridge. If constrained tightly enough to reproduce a plausible tracer field, the model cannot drift into solutions with tracer extrema.

A compromise between these two different approaches is decided upon: because we do not know what the true salinity field looks like, we smooth the model salinity distribution heavily to make it look as homogeneous as possible. The uncertainty in the a priori estimate of the salinity distribution is set to be very large so that the smoothing can act effectively. For $\delta^{18} \mathrm{O}$ and $\delta^{13} \mathrm{C}$ on the other hand, we have data from the Eastern Atlantic so we extrapolate them to the western basin where there are not many data points. The uncertainties in the extrapolated $\delta^{18} \mathrm{O}$ and $\delta^{13} \mathrm{C}$ values (for grid points which are not constrained by observations) are taken to be as small as the observational uncertainties $(0.2 \%$, see section II-C) in order to prevent extrema in the tracer distribution. Our objective is to show that several circulations are consistent with the paleo-tracer field, so artificially constraining the data distribution is a sensible approach as long as such circulations can be found. Also, these artificial constraints help to improve the numerical conditioning of the Hessian matrix of the objective function.

Thus, the main source of difficulties in modeling the paleo-ocean is the scarcity of data. Problems which do not occur in the modern ocean simulation are encountered because there the tracer data are not tightly constrained and there are no observations in large areas of the ocean. 
These problems for the paleo-ocean can be overcome by smoothing or extrapolating the tracer field.

\section{2) Run [1]: smallest changes to the modern ocean circulation necessary to make it consistent with the Last Glacial Maximum $\delta^{18} \mathrm{O}$ and $\delta^{13} \mathrm{C}$ data}

It is thought that the rate of formation of Lower North Atlantic Deep Water was reduced during the Last Glacial Maximum (see section II-C), but the extent of this reduction has not been determined. Thus the first experiment consists in trying to find the smallest changes in the modern ocean circulation and, more particularly, in the NADW rate of formation, necessary to fit the LGM data. The a priori estimate of the circulation is initialized to the modern ocean simulation. Since we are trying to find the smallest changes necessary, a large weight is put on this a priori estimate. Three tracers are modeled: $\delta^{18} \mathrm{O}$, which is converted to potential temperature (see section IV-3), salinity, and $\delta^{13} \mathrm{C}$. We use the results of the modern ocean run as a priori guess of the salinity distribution since there are no observations in the LGM ocean, and use a large uncertainty. The a posteriori salinity field is constrained to be very smooth. For $\delta^{18} \mathrm{O}$ and $\delta^{13} \mathrm{C}$, we have data obtained from the analysis of benthic foraminifera shells. We attribute an uncertainty of 0.2 per mil to the $\delta^{18} \mathrm{O}$ and $\delta^{13} \mathrm{C}$ data (see chapter II). Where no data are available, we extrapolate and attribute the same error bar of 0.2 per mil to the extrapolated values, so that the model does not drift into some unreasonable solution (see previous section).

The optimization exhibits a rather slow rate of convergence (Figure V-B-6) because of the lack of strong data constraints on the solution (see previous section). However, the optimization is quite successful. After inversion, mass is almost perfectly conserved. All model physical constraints are satisfied at better than $10 \%$ and at around $1 \%$ on average (Figure V-B-7).

The imbalances integrated over the whole model domain are very small. The integrated mass imbalances would take 170 million years to make the basin volume double, so more than one million years is necessary to change the volume by $1 \%$. As a comparison, the ocean volume changed by several \% in less than 10000 years during the last deglaciation. Thus the estimated 


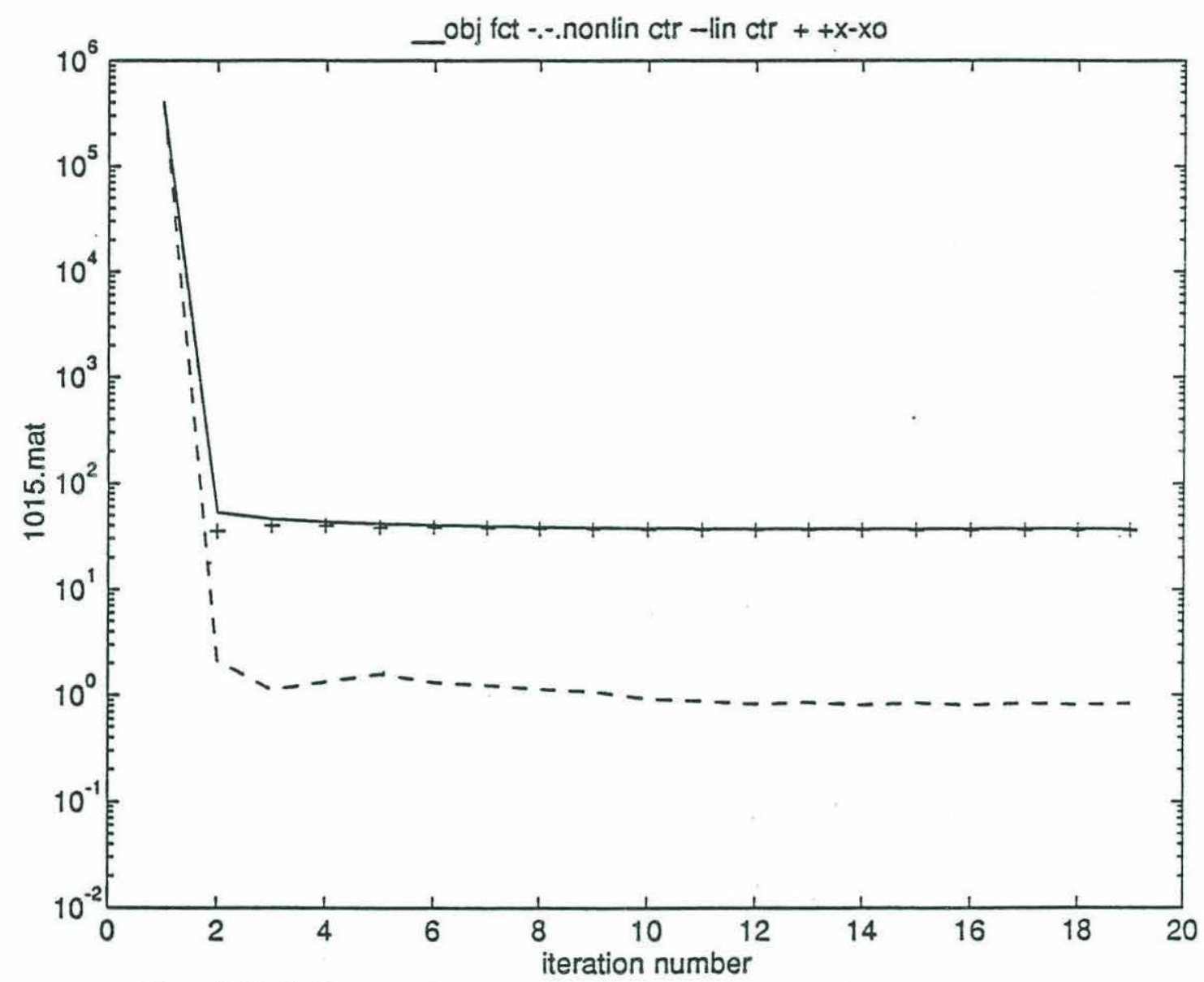

Figure V-B-6: Objective function versus number of iterations, for the LGM simulation I (full-strength NADW). Dashed line represents the contribution of the linear physical constraints to the objective function: $(A x-b)^{T} C_{A}{ }^{-1}(A x-b)$. Pluses represent the data misfit term $\left(\mathbf{x}-\mathbf{x}_{0}\right)^{\mathrm{T}} \mathrm{C}_{\mathbf{0}}^{-1}\left(\mathbf{x}-\mathbf{x}_{\mathbf{0}}\right)$. The initial data misfit is zero because the starting point of the minimization is the a priori solution, $\mathbf{x}_{\mathbf{0}}$. Full line represents the objective function, which is the sum of the two preceding terms. An infinite weight is put on the nonlinear physical constraints. They are not satisfied exactly though because the minimization is stopped after a limited number of iterations, and because of numerical round-off errors. 


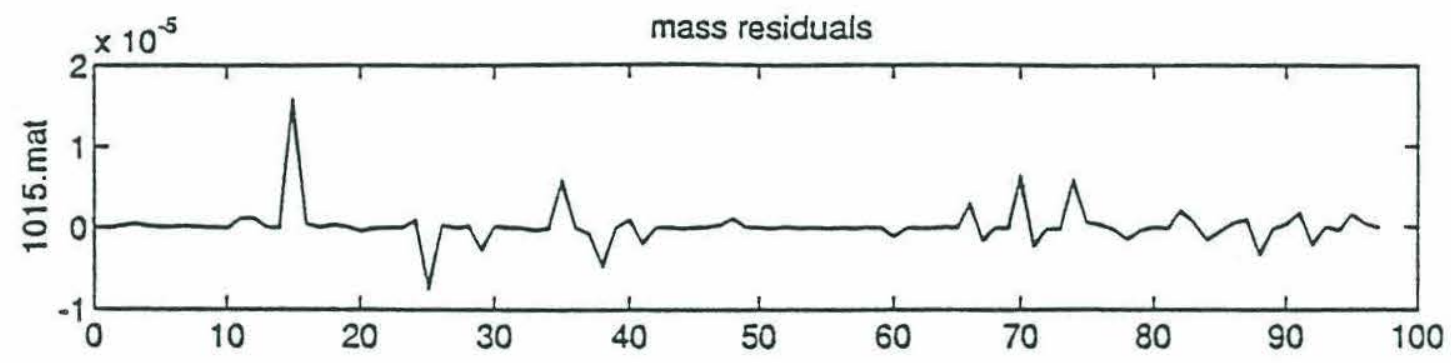

pot.temp. residuals

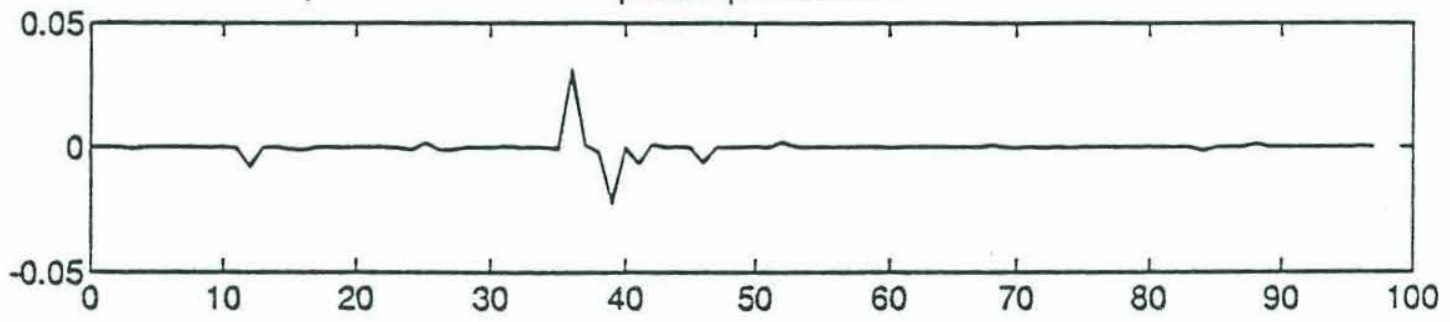

salinity residuals

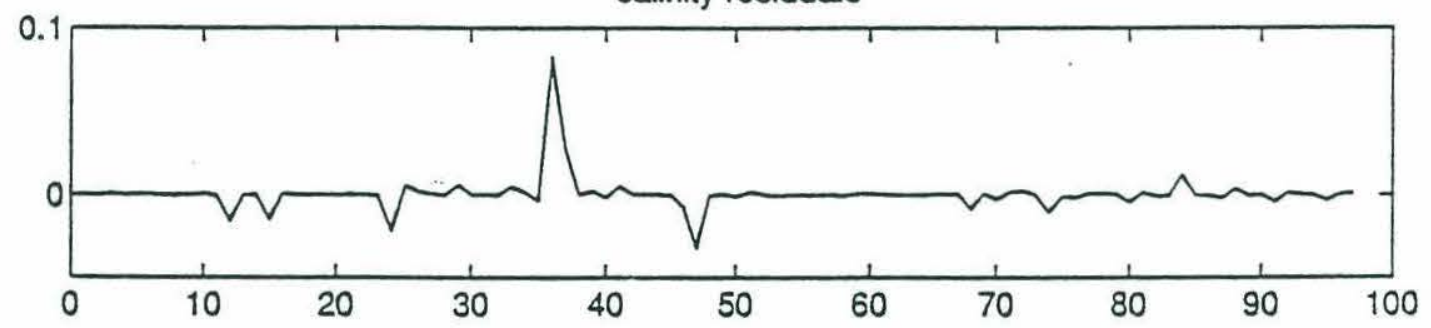

carbon 13 residuals

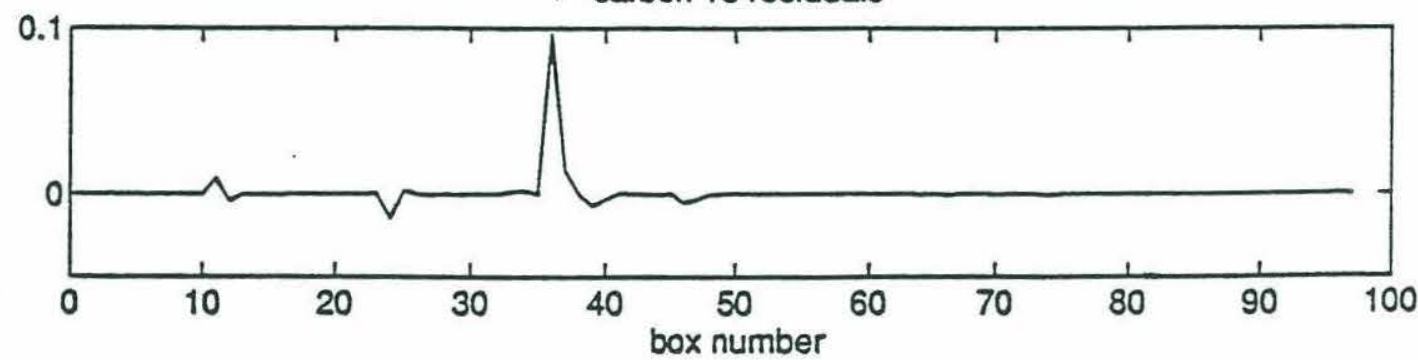

Figure V-B-7-a: Residuals after minimization in each volume element of the model, for the LGM simulation I (full-strength NADW). There are 97 such elements (boxes). The residuals are defined as the sum of the fluxes entering the box divided by the absolute value of the largest flux leaving or entering the box. 

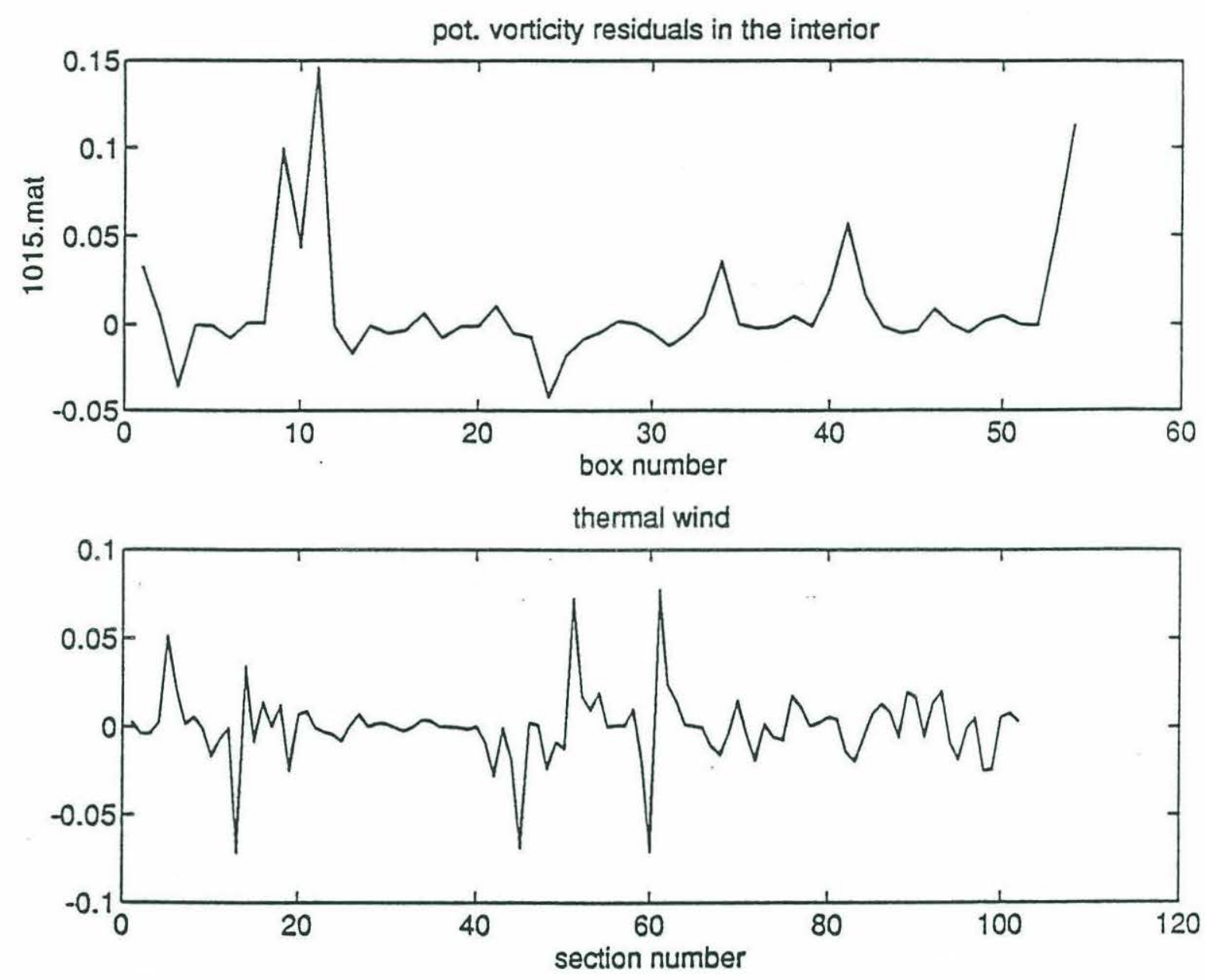

Figure V-B-7-b: Upper panel: Residuals after minimization of the linear vorticity balance in each box where it is imposed. Lower panel: Residuals after minimization of the thermal wind balance for each section where it is imposed. LGM simulation I (full-strength NADW). The residuals are defined as the left hand side minus the right hand side of the equations, divided by the largest of those two terms. 
circulation can be considered to be in steady state. The tracer imbalances would make the model average potential temperature vary by $1^{\circ} \mathrm{C}$ in 2 million years, the average salinity by 1 PSS in 20 million years, and the mean $\delta^{13} \mathrm{C}$ by $1 \%$ in 2 million years. These characteristic times are very large and show that the imbalances do not affect much the solution on the basin scale.

An important result of this first simulation is that one does not have to modify the modern ocean circulation much in order to accommodate the LGM tracer data. The circulation inferred in this first run is almost identical to the modern ocean circulation (compare Figures V-B-8a\&b to Figures V-A-6-a\&b). The maximum difference between the glacial and the modern zonally integrated transports across $50^{\circ} \mathrm{N}$ is $0.7 \mathrm{~Sv}$, in the deepest layer of the model (the zonally integrated transports across $10^{\circ} \mathrm{N}$ and $50^{\circ} \mathrm{N}$ are shown in Figure V-B-19 for the modern ocean run, and in Figure V-B-21 for the LGM run [1]). The zonally and vertically integrated transport between 1 and $5 \mathrm{~km}$ depth across $30^{\circ} \mathrm{N}$ is $15.7 \mathrm{~Sv}$ in the modern ocean run and $16.6 \mathrm{~Sv}$ in the LGM run [1]. It is only when looking at the details that differences become apparent. The exchange of mass across $10^{\circ} \mathrm{N}$ is very similar to that of the modern ocean run. Yet in the upper layer, there is slightly less water going north in the LGM run (Figures V-B-19\&21). The southward transport between 2 and $3 \mathrm{~km}$ depths goes from $4.3 \mathrm{~Sv}$ in the modern ocean up to 5.1 Sv during the LGM, and the southward transport between $4 \mathrm{~km}$ and $5 \mathrm{~km}$ depths goes from $7.1 \mathrm{~Sv}$ in the modern ocean down to $5.8 \mathrm{~Sv}$ during the LGM (Figures V-B-19\&21). This shift goes in the direction of a reduction of the flux of Lower NADW, but is quite small. Moreover, the northward volume transport across $10^{\circ} \mathrm{N}$ in the deepest level of the model goes from $0.6 \mathrm{~Sv}$ down to $0.4 \mathrm{~Sv}$ in the Western Atlantic, and from $1 \mathrm{~Sv}$ down to $0.25 \mathrm{~Sv}$ in the Eastern Atlantic. This reduction of the flux of water coming from the South Atlantic suggests a reduction of the flux of $\mathrm{AABW}$. The reduction of the flux of AABW seems proportionally larger than the reduction of the flux of NADW, which contradicts the conventional interpretation of the data (which argues for a reduced flux of NADW, the flux of $\mathrm{AABW}$ being fixed, or more generally a reduction of the ratio of the flux of NADW over the flux of AABW; Boyle and Keigwin, 1982).

The tracer field obtained after inversion is slightly different from the original distribution (Figure V-B-9\&10). However it looks reasonable, and is consistent with the data within the estimated uncertainty (Figure V-B-11). The departure from the observations does not follow a 

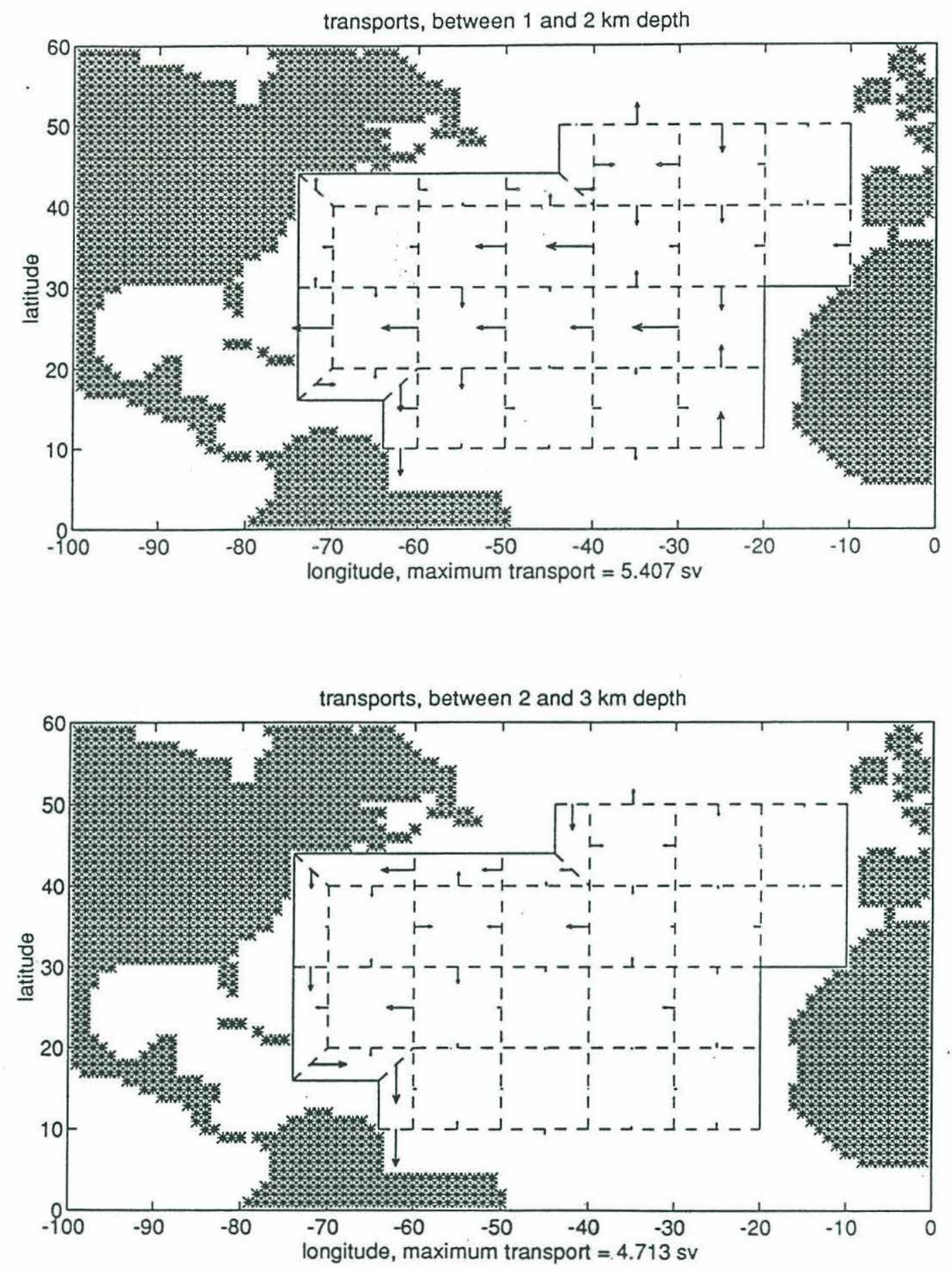

Figure V-B-8-a: Horizontal transports (in Sv) in the upper layers of the model, in the LGM simulation I (full-strength NADW). The transports associated with the longest arrow is indicated below each panel. The vectors in the westernmost boxes represent the transports in the DWBC. 

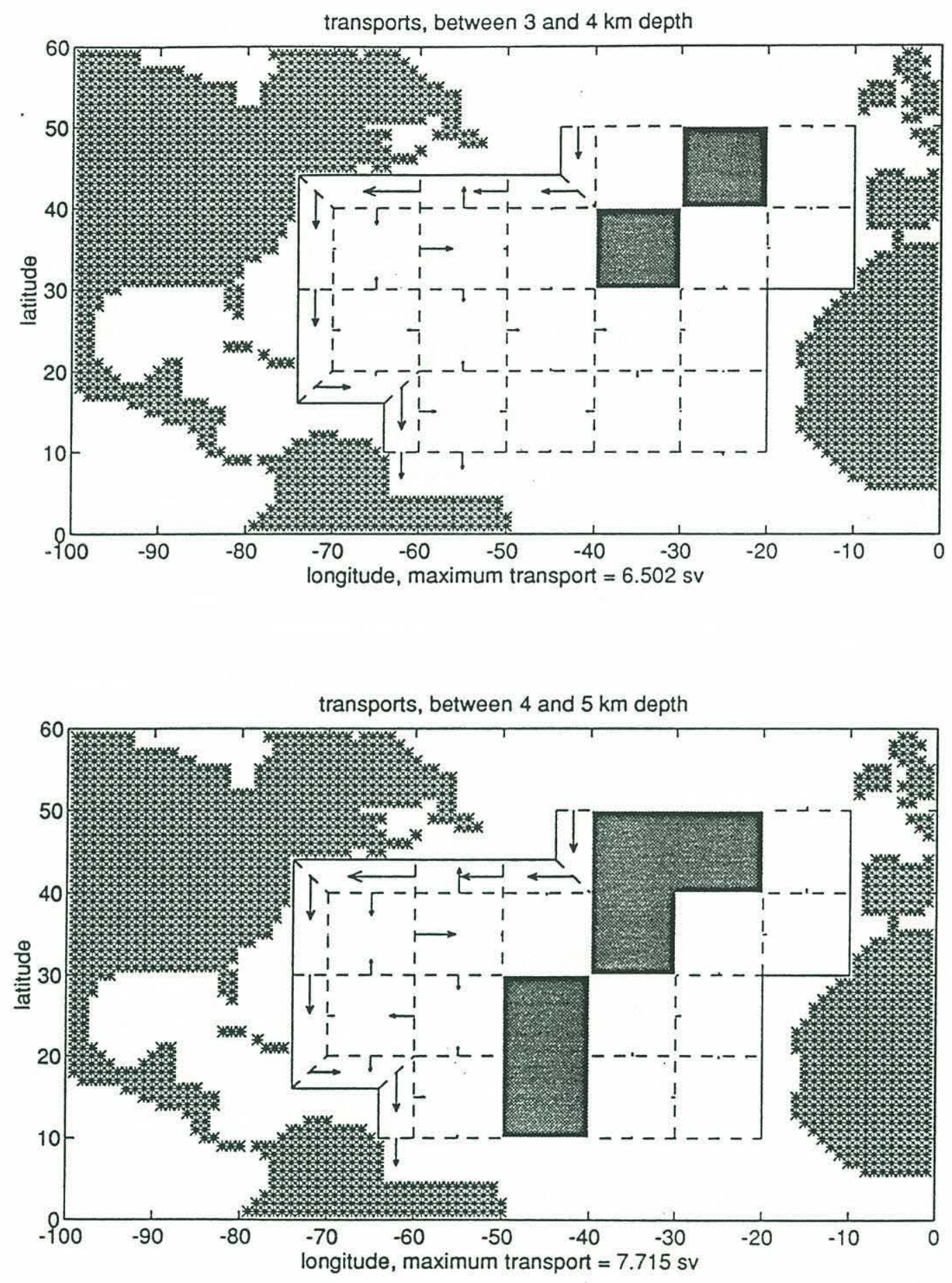

Figure V-B-8-b: Horizontal transports (in Sv) in the lower layers of the model, obtained in the LGM simulation I (full-strength NADW). The transports associated with the longest arrow is indicated below each panel. The vectors in the westernmost boxes represent the transports in the DWBC. 

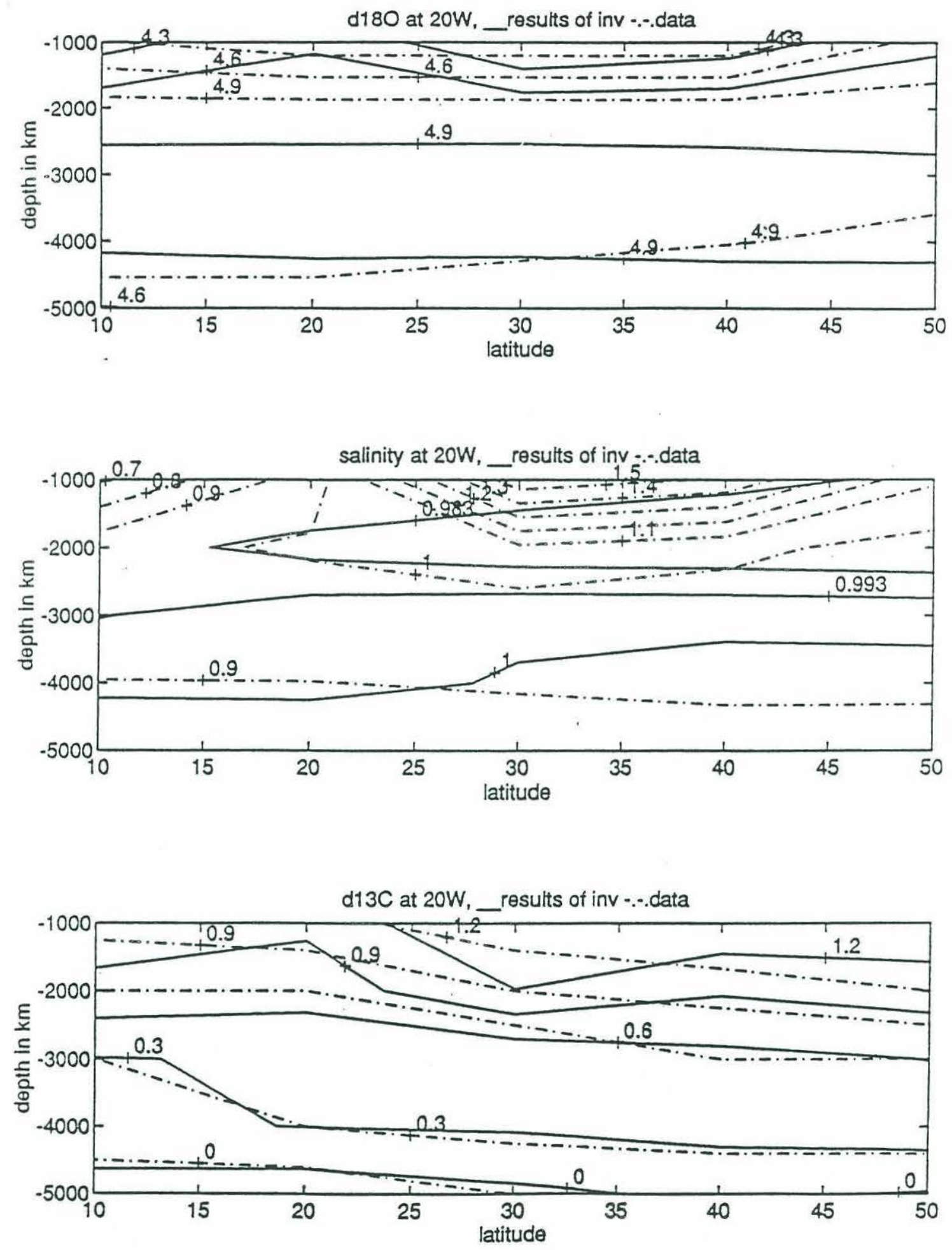

Figure V-B-9: Meridional sections of $\mathrm{d}^{18} \mathrm{O}(\% \circ)$, salinity (PSS-34), and $\mathrm{d}^{13} \mathrm{C}(\% \circ)$ in the LGM ocean at $20^{\circ} \mathrm{W}$. Full lines represent model predictions, LGM simulation I (full-strength NADW). Dot-dash lines represent the a priori tracer distributions. Note that the a posteriori salinity distribution is almost homogeneous. 

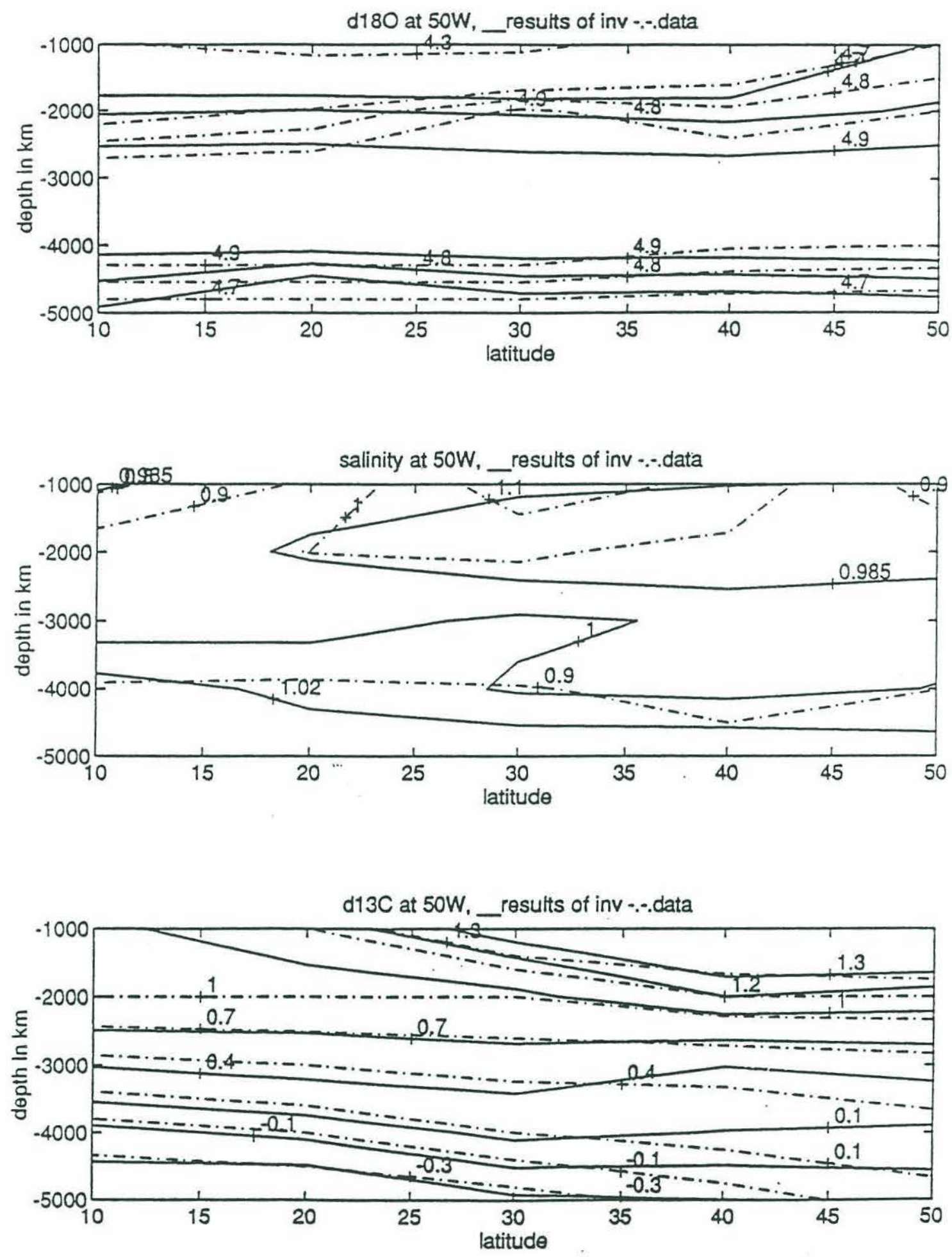

Figure V-B-10: Meridional sections of $\mathrm{d}^{18} \mathrm{O}(\%)$, salinity (PSS-34), and $\mathrm{d}^{13} \mathrm{C}(\%)$ in the LGM ocean, as in Figure V-B-9, except for $50^{\circ} \mathrm{W}$. Full lines represent model predictions, LGM simulation I (full-strength NADW). Dot-dash lines represent the $a$ priori tracer distributions. Note that the a posteriori salinity distribution is almost homogeneous. 


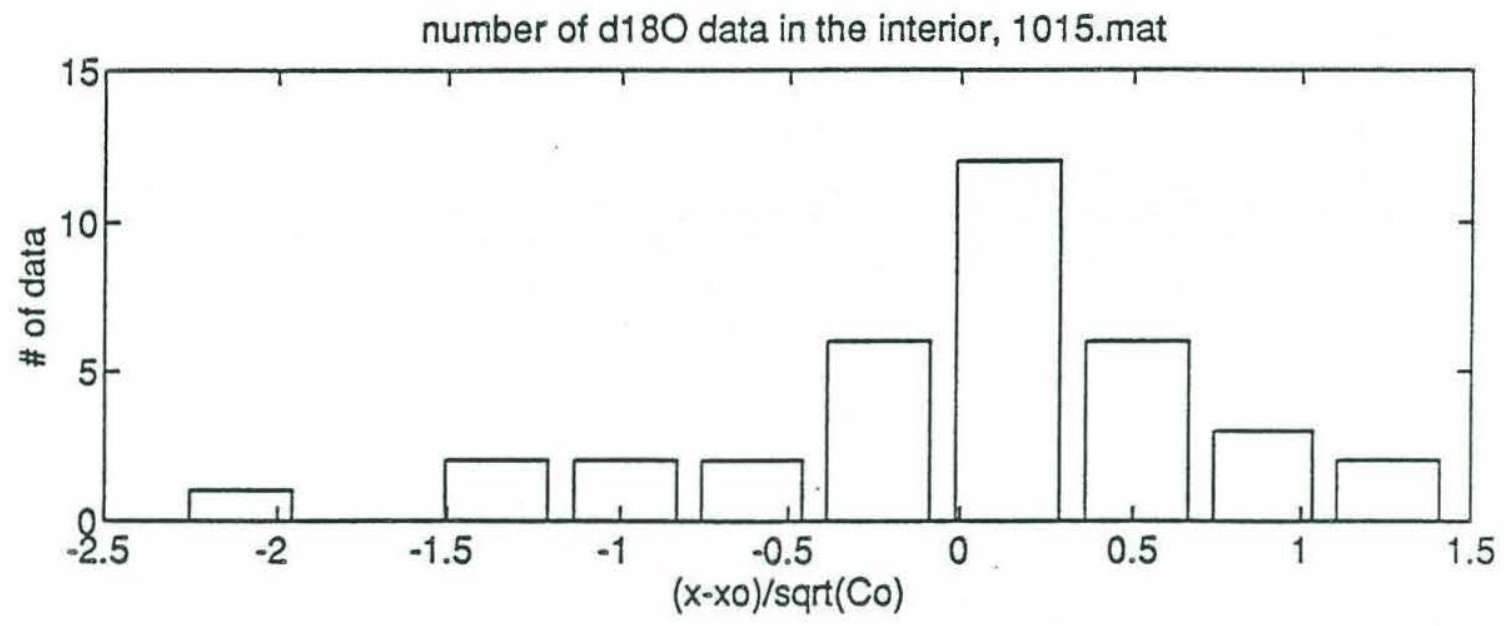

number of $d 13 \mathrm{C}$ data in the interior

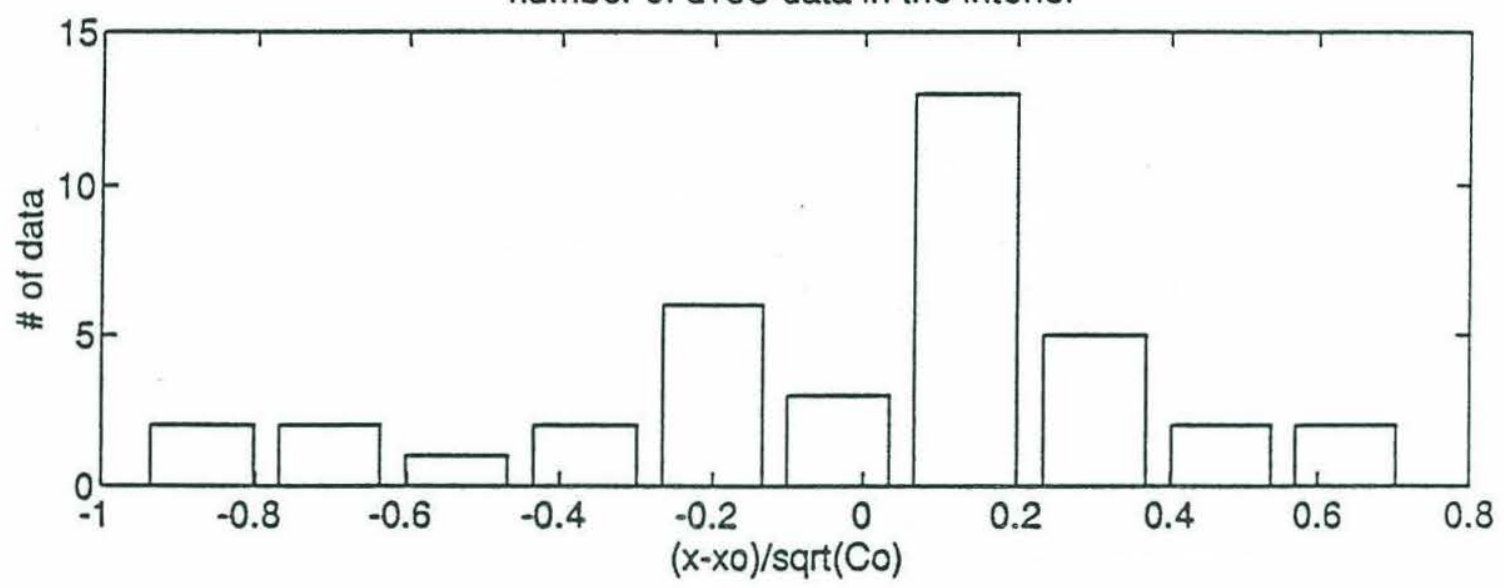

Figure V-B-11: Histogram of the data misfits after minimization for $\mathrm{d}^{18} \mathrm{O}$ and $\mathrm{d}^{13} \mathrm{C}$. LGM simulation I (full-strength NADW). Data misfit defined as tracer value predicted by the model minus a priori tracer value. Abscissa: data misfit divided by the standard deviation of the uncertainty in the a priori estimate (taken to be $0.2 \%$ o for both $\mathrm{d}^{18} \mathrm{O}$ and $\mathrm{d}^{13} \mathrm{C}$, see section $\mathrm{II}-\mathrm{C}$ ). Ordinate: number of data points in each interval of the histogram. 
normal distribution, but most of the departures are close to zero and only a few data misfits are larger than one standard deviation $(0.2 \%)$. In the absence of better information, the observational uncertainties are assumed to be the same for all the sediment cores. This assumption is unrealistic because a large fraction of the uncertainties is due to bioturbation (see chapter II), which varies geographically.

The a posteriori salinity field is almost homogeneous (Figure V-B-9\&10); its range of variation is about $0.02 \mathrm{PSS}$ at $20^{\circ} \mathrm{W}$. This is due to the large smoothing imposed in the model. One might argue that an almost completely homogeneous salinity field is not very realistic. However, this is the simplest solution one can imagine and there are no data to contradict it. Without smoothing, the inverted salinity would look extremely complicated with all kinds of structures, in which we would have no reason to believe.

As discussed in chapter $\Pi$, it is not clear what the latitudinal distribution of $\delta^{13} \mathrm{C}$ should look like. Different contouring of the data are possible. Labeyrie et al (1992) have proposed a contouring with a C-shape transition zone in the North Atlantic between waters of southern origins and waters formed in the North Atlantic (see chapter II). Another reconstruction by Lohmann and Lohmann (1994) suggests a vertically stratified distribution of $\delta^{13} \mathrm{C}$ (chapter II). The a priori estimate of the ${ }^{13} \mathrm{C}$ distribution in the model, as well as the a posteriori estimate, is closer to the second reconstruction. The two reconstructions are actually not very different in the North Atlantic. In both reconstructions, the most robust feature is the low $\delta^{13} \mathrm{C}$ values below 3$4 \mathrm{~km}$ depth. This has been considered to be the main argument in favor of a stronger source of AABW or a reduced source of Lower NADW during the LGM. However, the model can reproduce these low $\delta^{13} \mathrm{C}$ values without significantly reducing the flux of Lower NADW. In other words, this model simulation suggests that the modern ocean circulation is consistent with the LGM $\delta^{13} \mathrm{C}$ and $\delta^{18} \mathrm{O}$ data, and that large circulation changes are not required by these data. We will discuss this important result in part $\mathrm{C}$ of this chapter, but first let us examine other runs which will help understanding the problem. 


\section{3) Run [2]: rate of formation of Lower NADW reduced to half its modern ocean value}

We want to determine if circulations other than that obtained in run [1] can be found to be consistent with the paleo-data. Thus, we try a circulation with a significantly reduced rate of formation of Lower NADW (as represented by reduced zonally integrated southward transports across $50^{\circ} \mathrm{N}$ in the 2 lowest layers of the model). This reduced flux of Lower NADW scenario has become the conventional interpretation of the $\delta^{13} \mathrm{C}$ data (Boyle and Keigwin, 1987; Duplessy et al., 1988).

The a priori estimate of the tracer field is given by the paleo-data (as in run [1]) and the circulation is the same as in the modern ocean in the interior but with the DWBC strength reduced by $50 \%$. As in run [1], a large weight is put on this a priori estimate of the circulation so that the model modifies the $a$ priori transports as little as possible.

Here again the minimization is quite successful (Figure V-B-12). After inversion, mass is essentially perfectly conserved with residuals smaller than $2 \times 10^{-3} \%$ everywhere (Figure V-B-13). The maximum tracer residuals are less than $10 \%$, and the mean of the residuals of the order of a few tenths of a \%. Linear vorticity and thermal wind balances are well satisfied with maximum residuals less than about $10 \%$ and with mean residuals of roughly $1 \%$. The characteristic time for a doubling of North Atlantic volume due to integrated mass imbalances is 200 million years. Potential temperature would change by $1^{\circ} \mathrm{C}$ in 3 million years, salinity by 1 PSS in 600 million years and $\delta^{13} \mathrm{C}$ by $1 \%$ in 4 million years because of the imbalances. Therefore these imbalances are not large enough to affect the global mass and tracer budgets.

The zonally integrated southward transport below $3 \mathrm{~km}$ depth across $50^{\circ} \mathrm{N}$ is $4.6 \mathrm{~Sv}$ instead of 9.4 Sv in the previous scenario, which suggests that the flux of Lower NADW has been reduced by about 50\% compared to the modern ocean solution (Figure V-B-21 and Figure V-B-23). There is a southward transport of about $8.1 \mathrm{~Sv}$ between depths of $2 \mathrm{~km}$ and $5 \mathrm{~km}$ across $10^{\circ} \mathrm{N}$, as distinct from the $14.3 \mathrm{~Sv}$ of the scenario described in the previous section (Figure V-B-21 and Figure V-B-23). This smaller flux is due to a weaker DWBC in the reduced production of Lower 


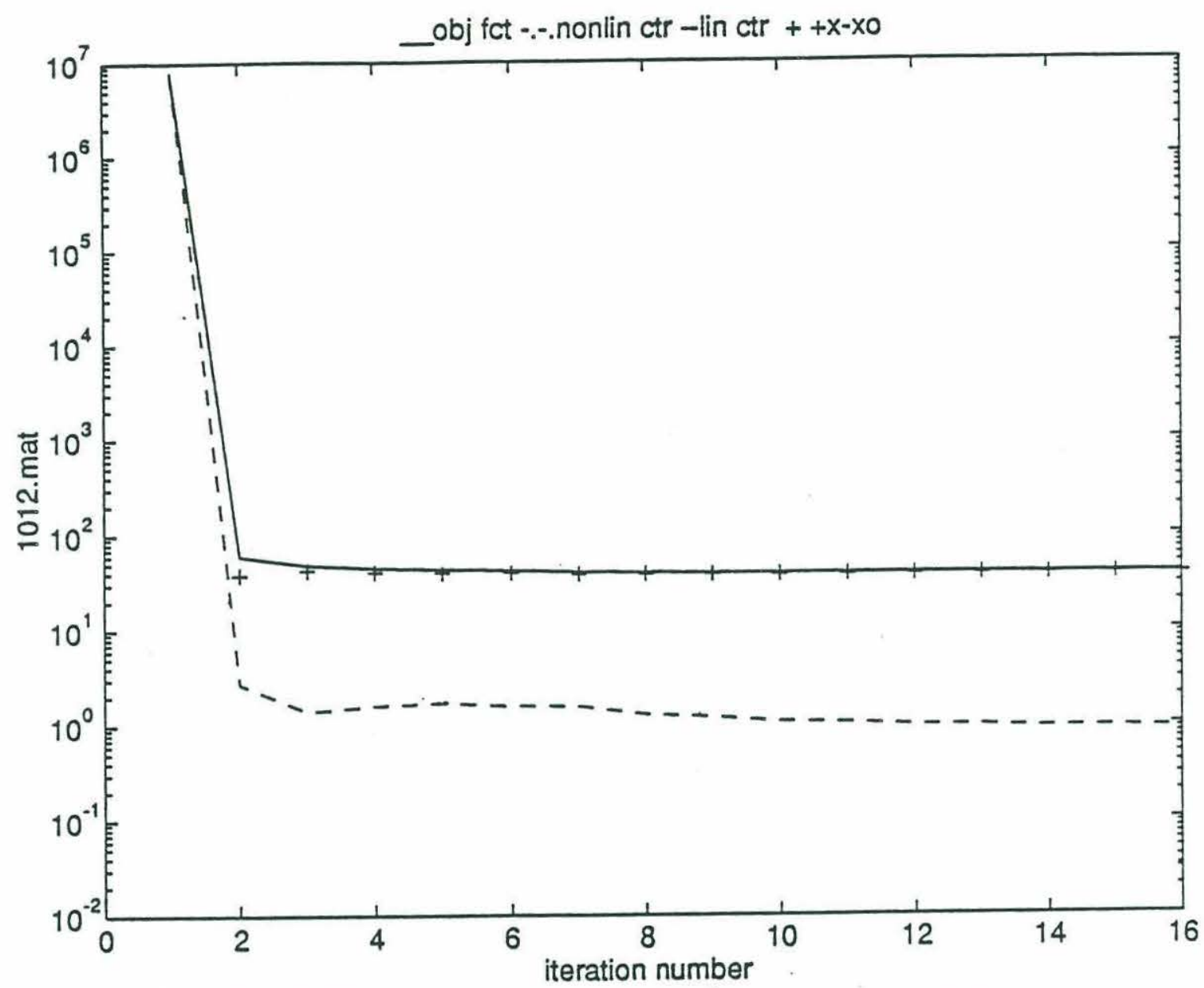

Figure V-B-12: Objective function versus number of iterations, for the LGM simulation II (reduced-strength NADW). Dashed line represents the contribution of the linear physical constraints to the objective function: $(A x-b)^{T} C_{A}{ }^{-1}(A x-b)$. Pluses represent the data misfit term $\left(x-x_{0}\right)^{T} C_{0}^{-1}\left(x-x_{0}\right)$. The initial data misfit is zero because the starting point of the minimization is the a priori solution, $x_{0}$. Full line represents the objective function, which is the sum of the two preceding terms. An infinite weight is put on the nonlinear physical constraints. They are not satisfied exactly though, because the minimization is stopped after a limited number of iterations, and because of numerical round-off errors. 

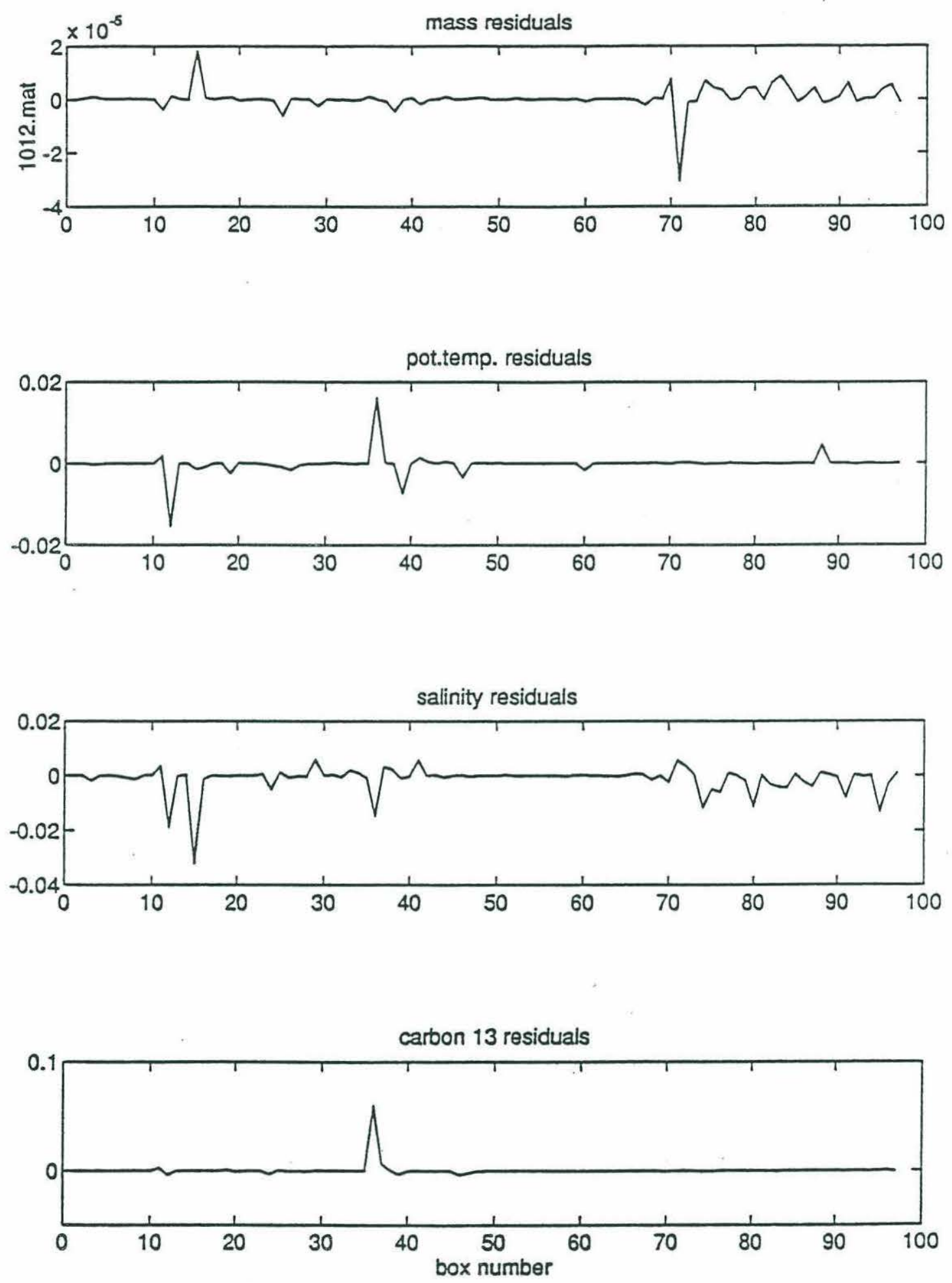

Figure V-B-13-a: Residuals after minimization in each volume element of the model, for the LGM simulation II (reduced-strength NADW). There are 97 such elements (boxes). The residuals are defined as the sum of the fluxes entering the box divided by the absolute value of the largest flux leaving or entering the box. 

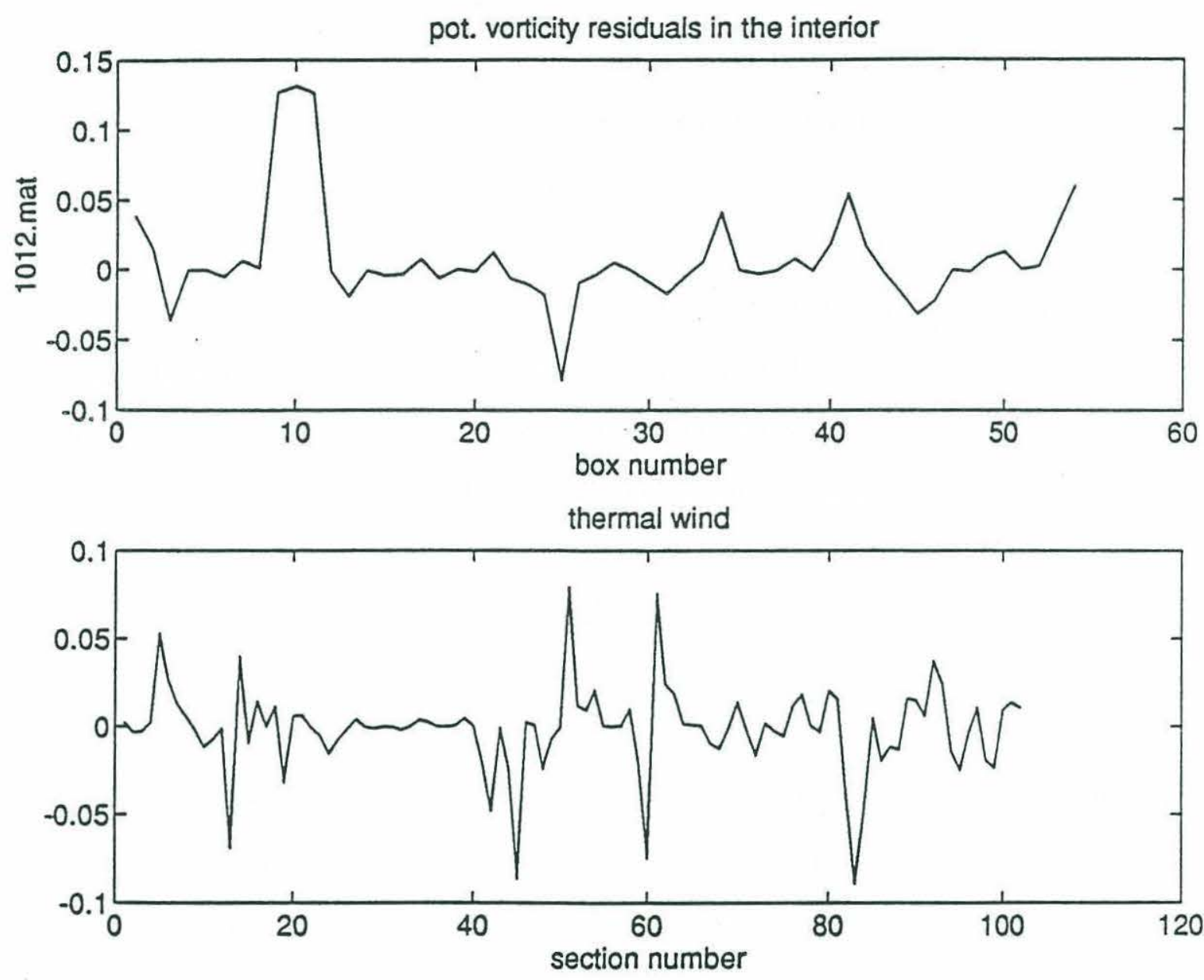

Figure V-B-13-b: Upper panel: Residuals after minimization of the linear vorticity balance in each box where it is imposed. Lower panel: Residuals after minimization of the thermal wind balance for each section where it is imposed. LGM simulation II (reducedstrength NADW). The residuals are defined as the left hand side minus the right hand side of the equations, divided by the largest of those two terms. 
NADW scenario; the interior fluxes are almost identical in both runs (compare Figures V-B-8-a\&b with Figures V-B-14-a\&b).

The $a$ posteriori estimates of the meridional profiles of tracers at $20^{\circ} \mathrm{W}$ and $50^{\circ} \mathrm{W}$ (Figure V-B15\&16) look plausible, and the tracer data are modified within their error bars (Figure V-B-17). Here again, the tracer departures from the data do not follow a normal distribution, which is not surprising in view of the problems associated with the data and their manipulation. Yet the data misfits are reasonably small and are deemed to be acceptable. The $a$ posteriori estimate of the tracer field looks very close to the one obtained with a full strength DWBC (compare Figures VB-9\&10 and Figures V-B-15\&16).

This simulation and the simulation described in the previous section show that different circulations, with different meridional transports of deep water, can produce tracer distributions consistent with the LGM $\delta^{13} \mathrm{C}$ and $\delta^{18} \mathrm{O}$ data. Thus, the interpretation of $\delta^{13} \mathrm{C}$ data in terms of oceanic circulation is ambiguous. We will see in section $\mathrm{C}$ why this is so.

\section{4) Are the results of the North Atlantic Model consistent with the South Atlantic data?}

The different simulations of the North Atlantic ocean described in the previous sections are internally consistent. They satisfy zero order physics and reproduce reasonably well the glacial North Atlantic data. However, one can wonder if these simulations are compatible with data outside the North Atlantic. Even though there are not nearly as many observations in the South Atlantic or the Pacific oceans as in the North Atlantic, they could still prove to be incompatible with the fluxes inferred from the North Atlantic model. More specifically, the $\delta^{13} \mathrm{C}$ values at the southern boundary of the North Atlantic model were lower during the LGM than today. These low values may be incompatible with the large flux of NADW obtained in run [1] because this flux brings waters to the South Atlantic richer in $\delta^{13} \mathrm{C}$ than Southern Ocean waters, and could potentially raise $\delta^{13} \mathrm{C}$ values in the South Atlantic above the observed values. To verify that this situation does not occur, it is necessary to extend the model simulations to the South Atlantic. 

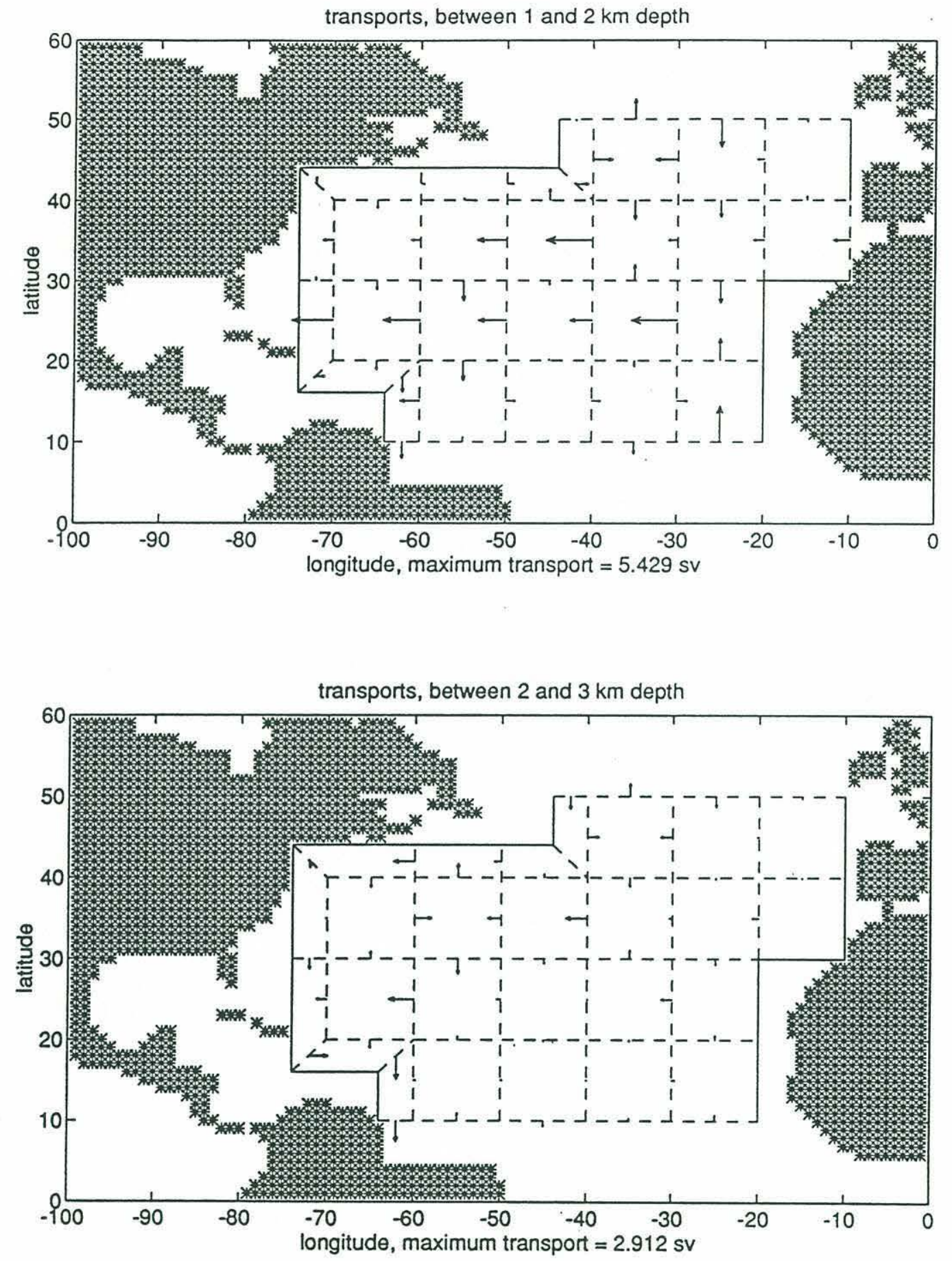

Figure V-B-14-a: Horizontal transports (in Sv) in the upper layers of the model, obtained in the LGM simulation II (reduced-strength NADW). The transport associated with the longest arrow is indicated below each panel. The vectors in the westernmost boxes represent the transports in the DWBC. 

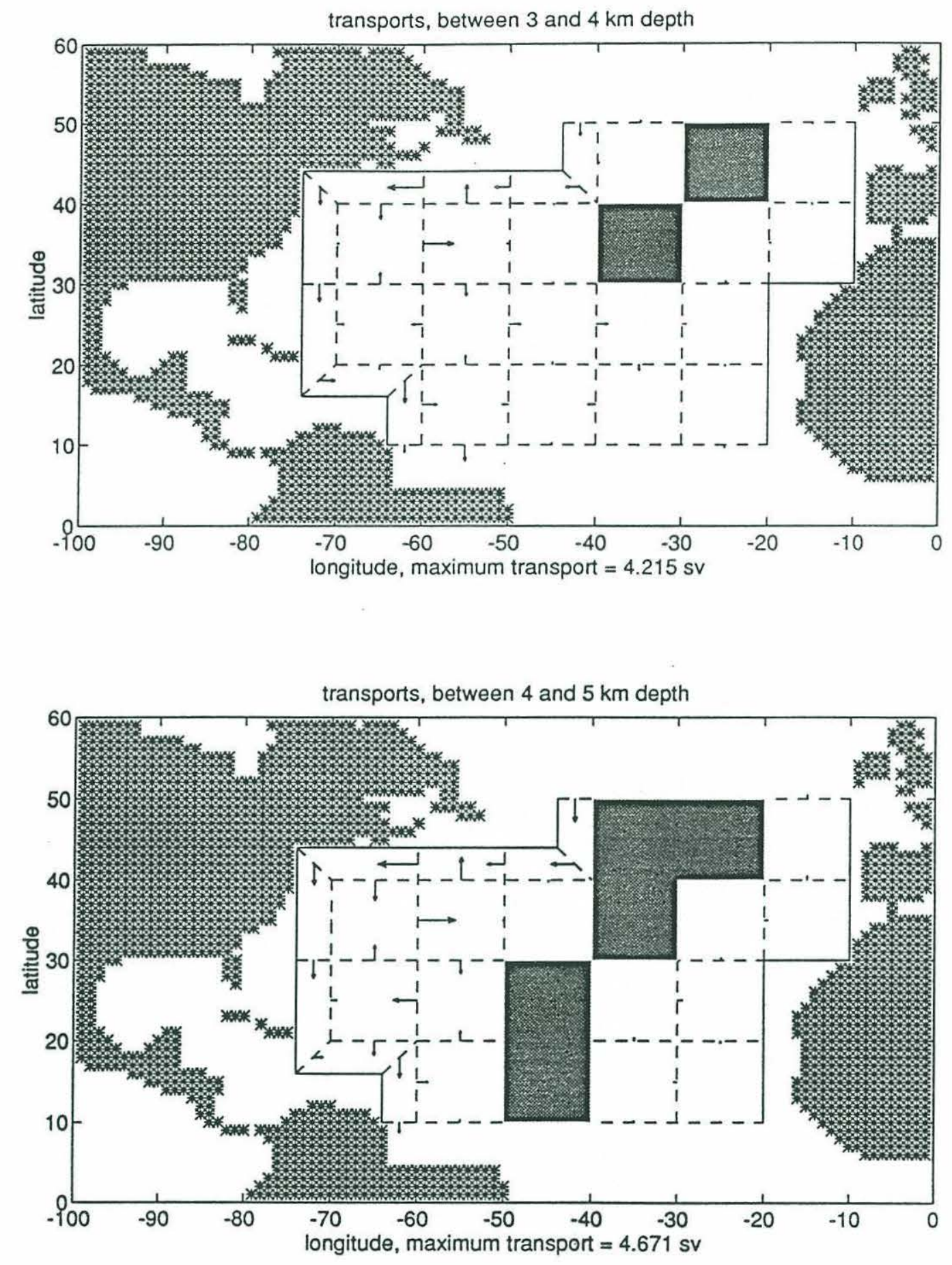

Figure V-B-14-b: Horizontal transports (in Sv) in the lower layers of the model, obtained in the LGM simulation II (reduced-strength NADW). The transport associated with the longest arrow is indicated below each panel. The vectors in the westernmost boxes represent the transports in the DWBC. 

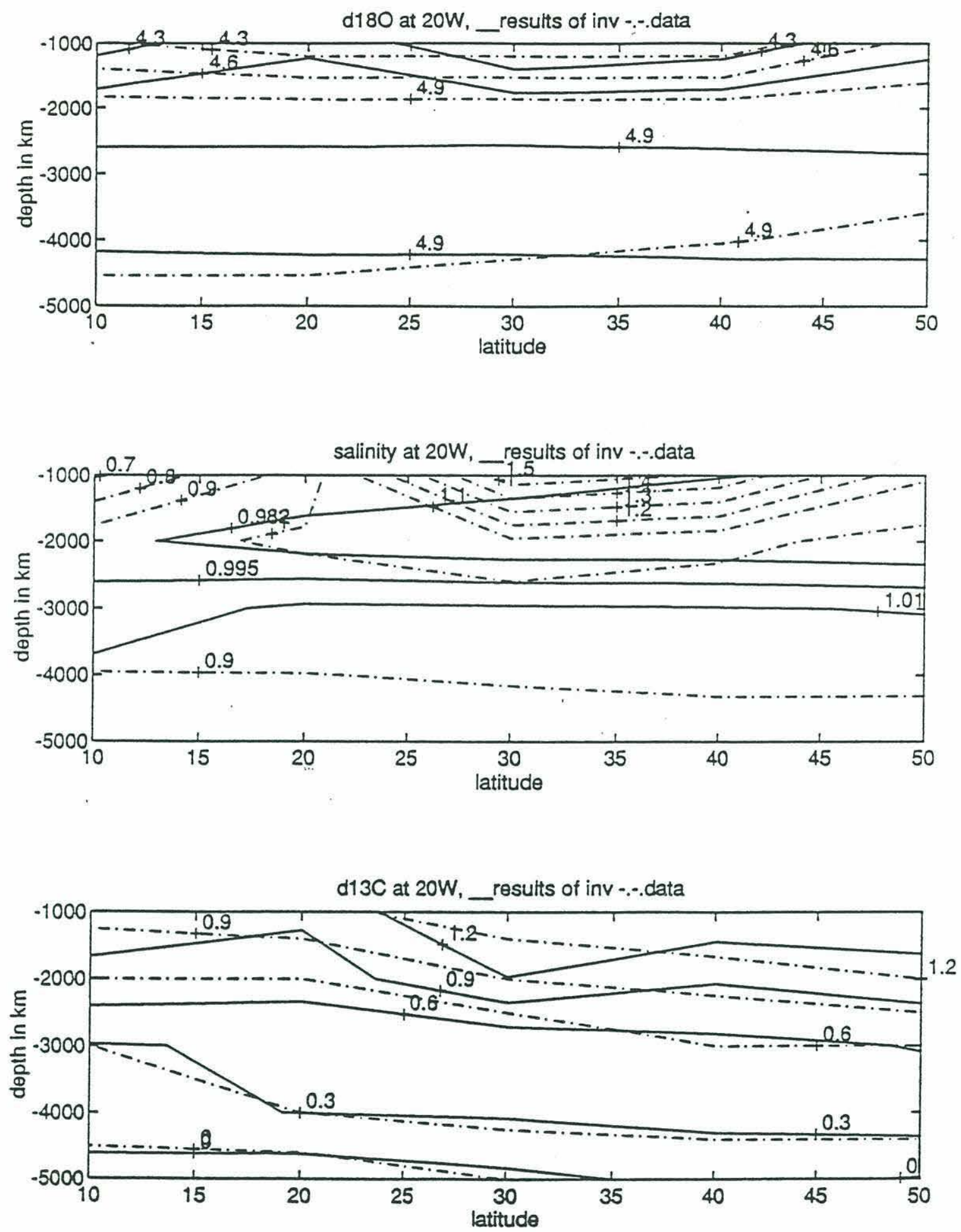

Figure V-B-15: Meridional sections of $\mathrm{d}^{18} \mathrm{O}(\%)$, salinity (PSS-34), and $\mathrm{d}^{13} \mathrm{C}(\% \circ)$ in the LGM ocean at $20^{\circ} \mathrm{W}$. Full lines represent model predictions, LGM simulation II (reduced-strength NADW). Dot-dash lines represent the a priori tracer distributions. Note that the a posteriori salinity distribution is almost homogeneous. 

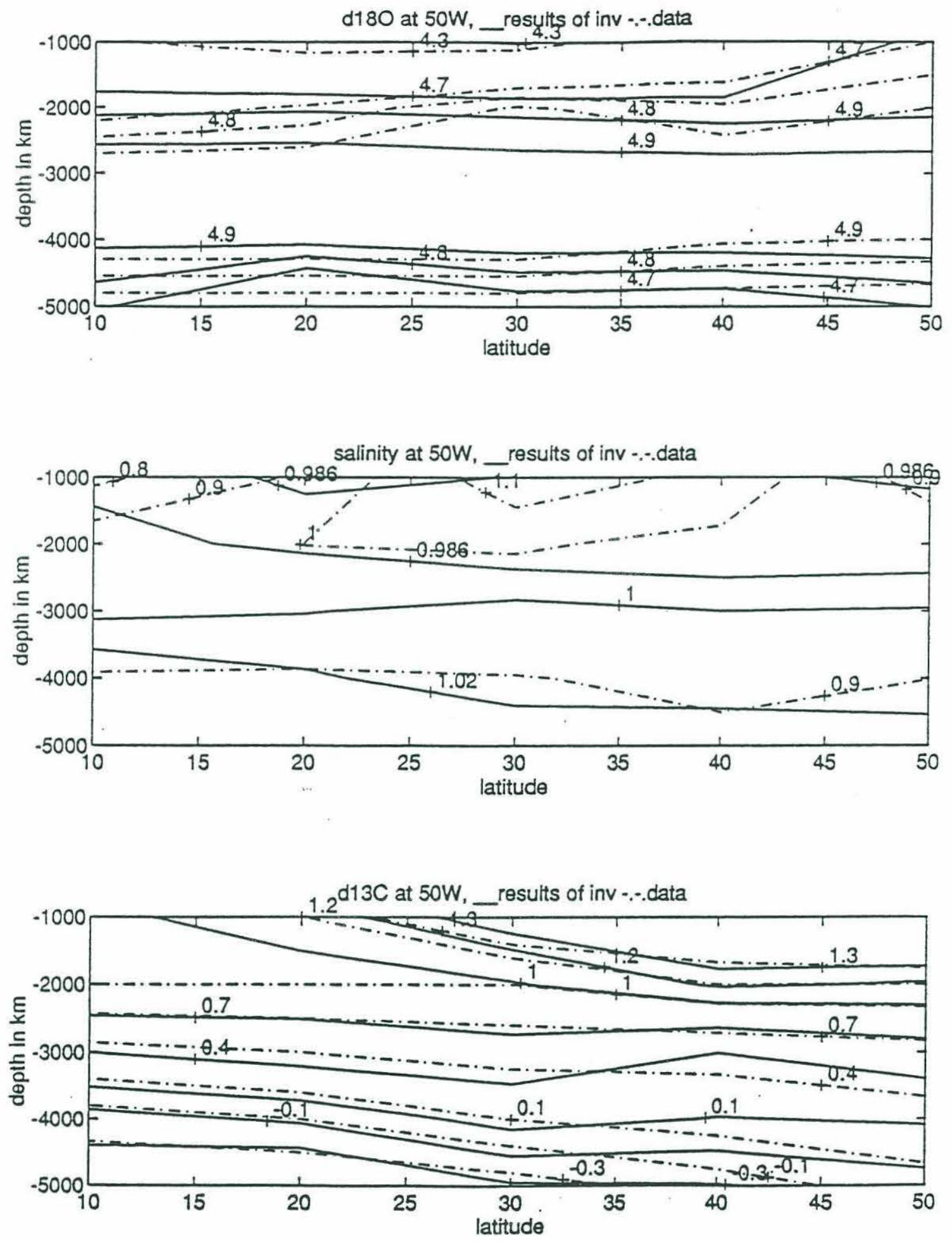

Figure V-B-16: Meridional sections of $\mathrm{d}^{18} \mathrm{O}(\%)$, salinity (PSS-34), and $\mathrm{d}^{13} \mathrm{C}(\%)$ in the LGM ocean, as in Figure V-B-14, except for $50^{\circ} \mathrm{W}$. Full lines represent model predictions, simulation II (reduced-strength NADW). Dot-dash lines represent the $a$ priori tracer distributions. Note that the a posteriori salinity distribution is almost homogeneous. 

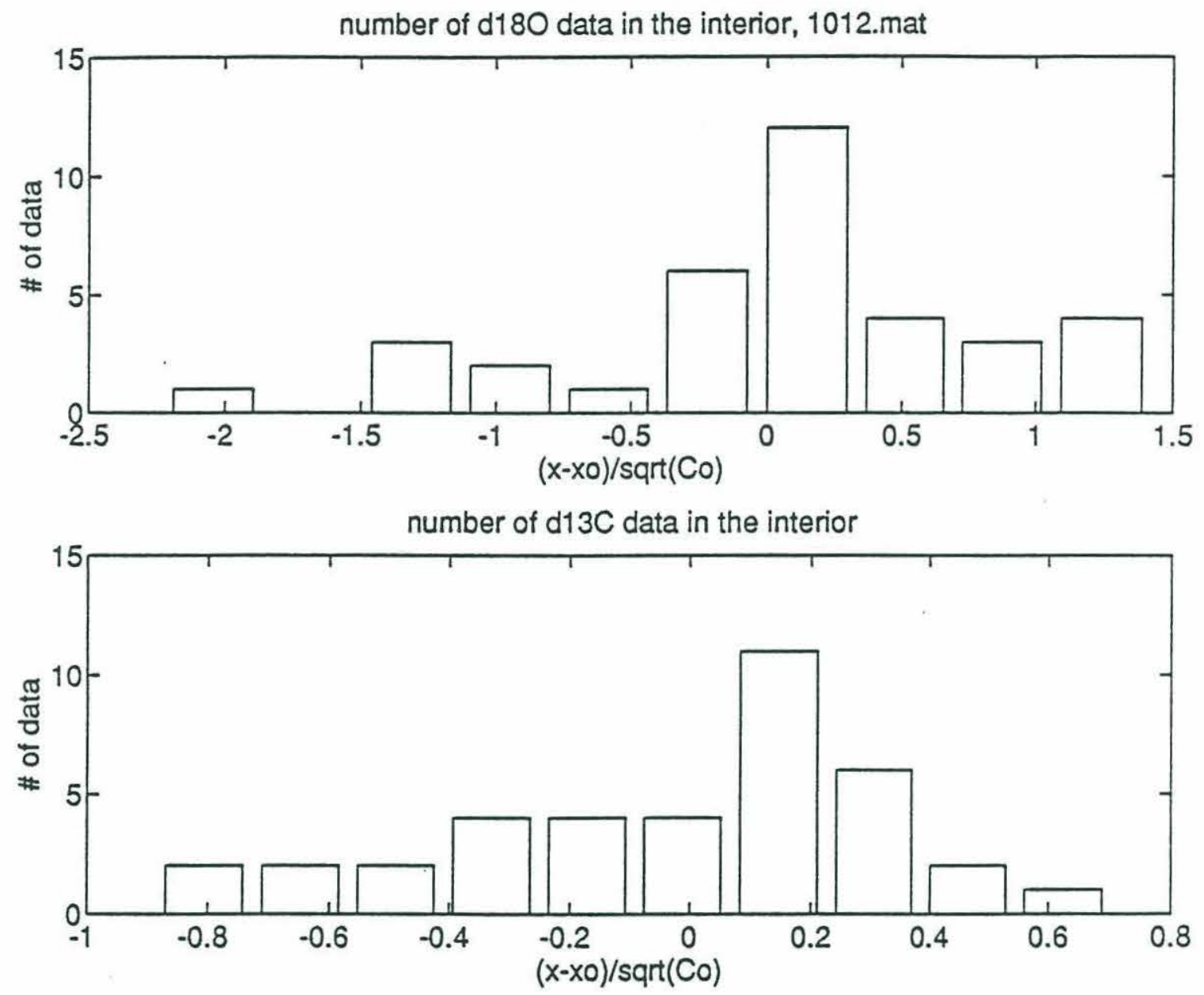

Figure V-B-17: Histogram of the data misfits after minimization for $\mathrm{d}^{18} \mathrm{O}$ and $\mathrm{d}^{13} \mathrm{C}$. LGM simulation II (reduced-strength NADW). Data misfit defined as tracer value predicted by the model minus a priori tracer value. Abscissa: data misfit divided by the standard deviation of the uncertainty in the a priori estimate (taken to be $0.2 \%$ o for both $\mathrm{d}^{18} \mathrm{O}$ and $\mathrm{d}^{13} \mathrm{C}$, see section II-C). Ordinate: number of data points in each interval of the histogram. 
Ideally, one would use a global model including all the oceans as well as exchanges with the atmosphere, and assimilate all the available data to insure that everything is consistent. However, to our knowledge, this has never been attempted for the modern ocean with a nonlinear inverse model, and is beyond the scope of this study for LGM simulations. Thus we limit ourselves to consistency checks in the South Atlantic. We will see that it is actually quite easy to find circulations compatible with the limited South Atlantic data base, which gives us some confidence that data from other basins could be accommodated as well.

Since there are few data points in the South Atlantic (compared to the North Atlantic), we use a very simple kinematic 5-box model to simulate the deep circulation (see Figure V-B-18).

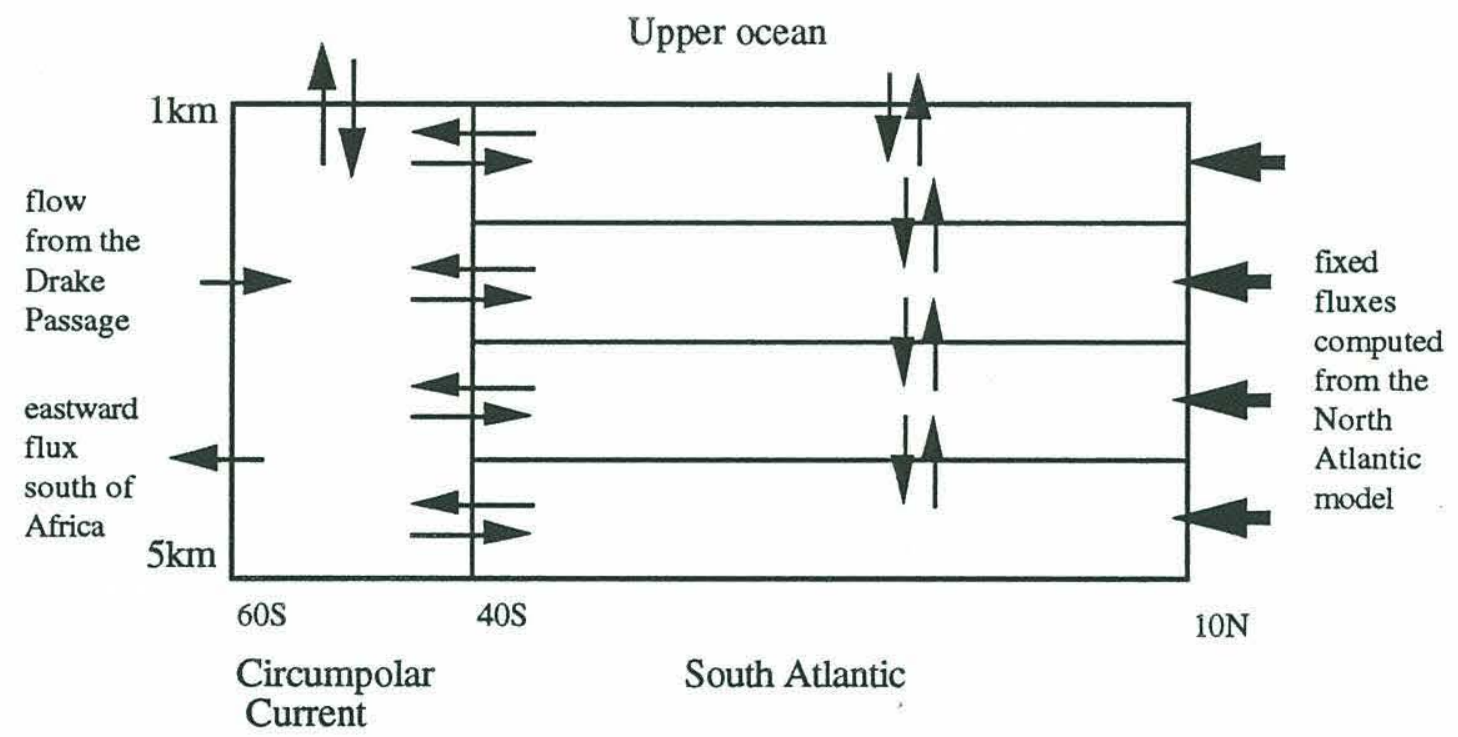

Figure V-B-18: 5-box model of the deep South Atlantic.

As in the North Atlantic model, the South Atlantic basin is divided into 4 layers in the vertical and the upper waters are not explicitly modeled. The Atlantic sector of the circumpolar current below $1 \mathrm{~km}$ depth is represented by one box. The waters entering this box through the Drake passage and leaving it past the South African peninsula are represented by 2 fluxes. There is exchange of water with the upper layers. The tracer characteristics of upper waters and Drake passage water, as well as the fluxes leaving and entering the model domain are treated as 
unknown variables and estimated as part of the solution. However, the fluxes of mass and tracers exchanged with the North Atlantic come from the North Atlantic model output and are fixed parameters, the goal being to check their consistency with the South Atlantic data.

Only mass, ${ }^{13} \mathrm{C}$, and potential temperature (computed from $\delta^{18} \mathrm{O}$ data) conservation are imposed. No dynamics can be included in such a crude model. Since there are no data for salt and we do not need to compute density (thermal wind is not imposed), salt conservation would not add any information on the solution and is not taken into account. The effect of biology is neglected. Were it to be taken into account, it would introduce more degrees of freedom in the model, which would make it easier to combine the fluxes computed from the North Atlantic model with the South Atlantic data.

The model appears mathematically as 15 equations (10 being nonlinear) in 36 unknowns (tracer concentrations and volume transports). Since it is a very small problem, it can be easily solved using constrained optimization techniques. We use a "minimax" approach (see chapter III) which consists in trying to minimize the maximum equation imbalance. This method is very convenient because it tends to minimize all the equation imbalances to the same level so the constraints are equally well satisfied in every box, and we avoid the problem of having large imbalances concentrated in a few boxes. Tracer concentrations are bounded by inequality constraints so there is no need for a data misfit term in the objective function. Formally, the optimization problem is:

$$
\begin{gathered}
\left.\min \mathrm{J}=\underset{\mathrm{i}=1: 15}{\min (\max } \mid \mathrm{f}_{\mathrm{i}}\left(\mathrm{x}_{1}, \ldots, \mathrm{x}_{36}\right)\right) \\
\mathrm{l}_{1} \leq \mathrm{x}_{1} \leq \mathrm{u}_{1} \\
\cdot \\
\cdot \\
\mathrm{l}_{36} \leq \mathrm{x}_{36} \leq \mathrm{u}_{36}
\end{gathered}
$$

It is somewhat difficult to determine what the data lower $\left(1_{1}, \ldots, 136\right)$ and upper bounds $\left(u_{1}, \ldots, u_{36}\right)$ should be for LGM simulations. There are significant problems with both the Southern Ocean and South Atlantic data (see chapter II). Mackensen et al. (1993) suggest that the LGM $\delta^{13} \mathrm{C}$ data should be about $0.6 \%$ o lower than modern values which gives a $\delta^{13} \mathrm{C}$ of about $-0.3 \%$ in the deep Circumpolar Current box. For the South Atlantic, there is a substantial 
disagreement between the data we compiled and Lohmann and Lohmann's (1994) data. The present compilation suggests very negative values near the bottom (as low as $-0.7 \%$ ) whereas Lohmann and Lohmann's data indicate values close to $0 \%$ for AABW. Similarly, the compilation indicates low $\delta^{13} \mathrm{C}$ near $1 \mathrm{~km}$ depth as opposed to large values of more than $1 \%$ in Lohmann and Lohmann's data. In light of Mackensen et al.'s results, we might expect the AABW data of the compilation to show values too negative because most data come from high productivity regions off the African coast. On the other hand, Lohmann and Lohmann's data indicate $\delta^{13} \mathrm{C}$ values at 1 $\mathrm{km}$ depth as high for the LGM as for the present time, which may be too high since there was a global shift of $\delta^{13} \mathrm{C}$ toward lower values during the LGM (Curry et al., 1988). Thus we take Lohmann and Lohmann's $\delta^{13} \mathrm{C}$ profile as a typical profile for the South Atlantic data, but we arbitrarily impose the limitation that the upper ocean value is less than $1 \%$. It is possible to find solutions consistent with lower $\delta^{13} \mathrm{C}$ values $(0.6 \%$ ) between 1 and $2 \mathrm{~km}$ depths as well (solutions not shown here).

In the following section, we check the consistency with South Atlantic data of the three different North Atlantic simulations described above.

\section{a/ Modern ocean simulation}

The fluxes through the northern boundary of the model are computed from the output of run [0] described in section V-A. The fluxes of tracer are computed across each box interface at $10^{\circ} \mathrm{N}$ in the North Atlantic model and then zonally integrated over the whole Atlantic section. Thus, the different components of the fluxes are not explicitly dealt with (see section V-A for detailed mass

fluxes at $\left.10^{\circ} \mathrm{N}\right)$. Note that the $\delta^{13} \mathrm{C}$ fluxes are computed by translating the phosphate concentrations into $\delta^{13} \mathrm{C}$ (see $\left.\mathrm{V}-\mathrm{A}\right)$.

There is net upwelling everywhere in the South Atlantic boxes (see Figure V-B-19). 19.4 Sv flow from the South Atlantic into the Circumpolar Current between 2 and $5 \mathrm{~km}$ depth. $9.2 \mathrm{~Sv}$ go northward across $40^{\circ} \mathrm{S}$ in the bottom layer. The upper layer of the South Atlantic carries $4.1 \mathrm{~Sv}$ northward across $40^{\circ} \mathrm{S}$, which is consistent with the idea of northward spreading of AAIW in the intermediate layers of the ocean. We assign a transport of about $50 \mathrm{~Sv}$ to the circumpolar current at depths below $1 \mathrm{~km}$. It is not clear what this number should exactly be, but $50 \mathrm{~Sv}$ is about $1 / 3$ of 


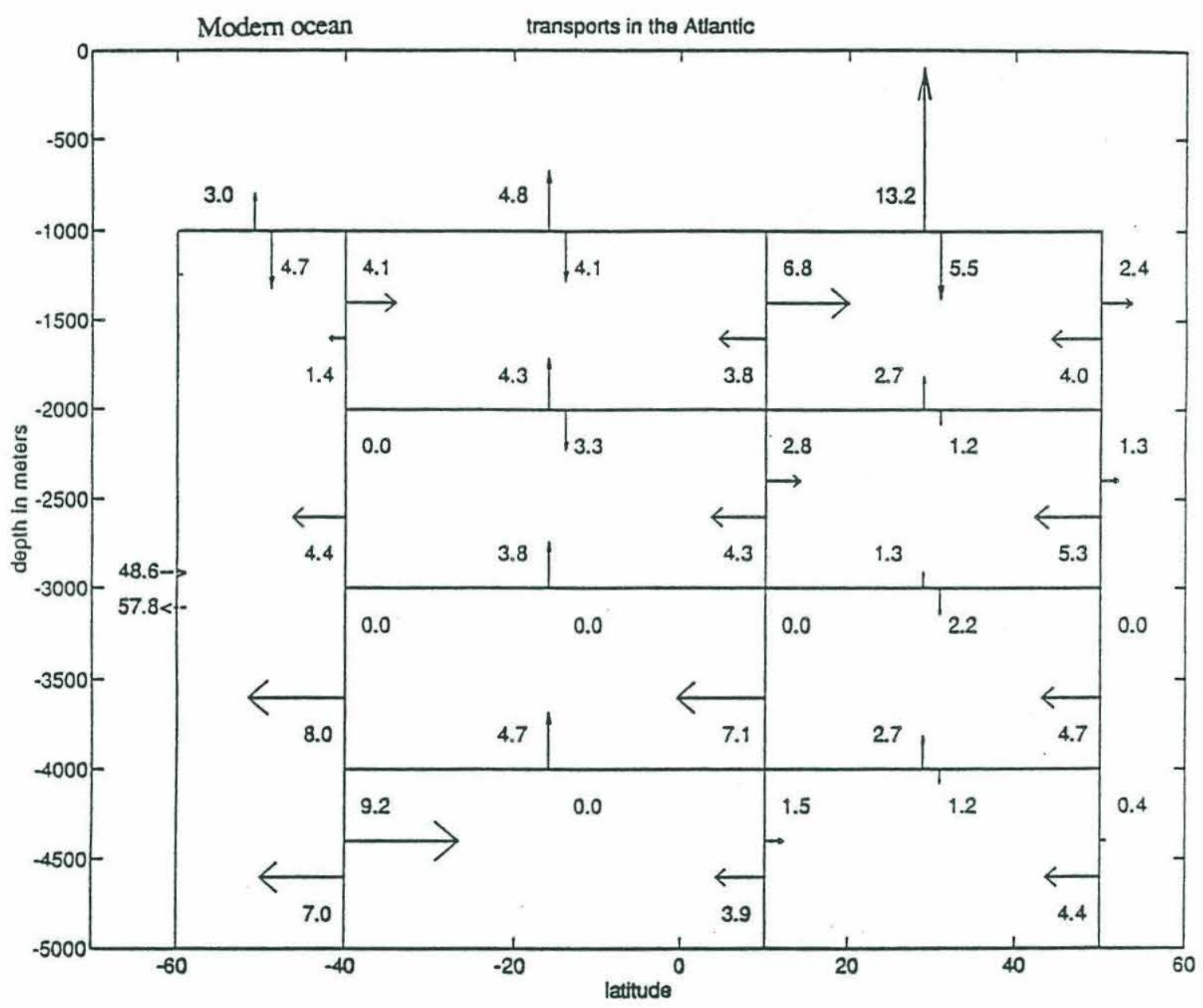

Figure V-B-19: Northward and southward zonally integrated transports (in Sv) in the modern Atlantic. Fluxes north of the equator estimated by dynamical box model (97 boxes). Fluxes south of the equator estimated by kinematic 5-box model (forced with fluxes across $10^{\circ} \mathrm{N}$ computed by dynamical box-model). Volume fluxes into and out of Atlantic sector of Circumpolar Current are in reality perpendicular to the meridional plan. The arrows corresponding to these fluxes are not scaled. 
the vertically integrated transport which is consistent with the fact that most of the transport takes place in the upper km (Nowlin and Klink, 1986). Lower transports (around $20 \mathrm{~Sv}$ ) are also compatible with the model and the tracer data (solution not shown here).

The $\delta^{13} \mathrm{C}$ distribution in the South Atlantic (Figure V-B-20) appears to be reasonably close to Kroopnick's data (1985, see Figure II-B-2). Potential temperature variables are meant to reproduce the GEOSECS Western Atlantic data (1980). It is somewhat difficult to assign a value to each box (note that no attempt has been made to compute what the GEOSECS data would be if averaged over each box) but the $a$ posteriori tracer distribution looks reasonable (Figure V-B-20).

The potential temperature and ${ }^{13} \mathrm{C}$ conservation equations are reasonably well satisfied with residuals well below $1 \%$ (residuals not shown here). This simple box model shows that the fluxes of potential temperature and ${ }^{13} \mathrm{C}$ computed from the North Atlantic modern ocean simulation are not fundamentally incompatible with the South Atlantic data. One would need a more realistic model to conclude that they are fully consistent with all the South Atlantic data, but this is beyond the scope of this study.

\section{b/ LGM simulation of the South Atlantic with strong NADW formation}

The data available in the Ice Age South Atlantic are very few and somewhat contradictory (see chapter II), so using a sophisticated model to interpret the data is probably not justified. Mass, potential temperature (estimated from $\delta^{18} \mathrm{O}$ data) and ${ }^{13} \mathrm{C}$ conservation are imposed. The fluxes across the northern boundary of the model are computed from the output of run [1]. Because the South Atlantic tracer distribution is poorly constrained, tracer concentrations are relatively free to vary so that conservation equations are almost perfectly satisfied (residuals almost 0 , not shown here). Here again, there is net upwelling everywhere in the South Atlantic except at $2 \mathrm{~km}$ depth (Figure V-B-21). The transports to and from the Circumpolar Current are very similar to that of the modern ocean. The $\delta^{13} \mathrm{C}$ distribution is constrained to reproduce Lohmann and Lohmann's (1994) data, and looks reasonable (Figure V-B-22-a\&b). The potential temperature values are not constrained by observations in the LGM ocean, but appear to be plausible (Figure V-B-22-b). 

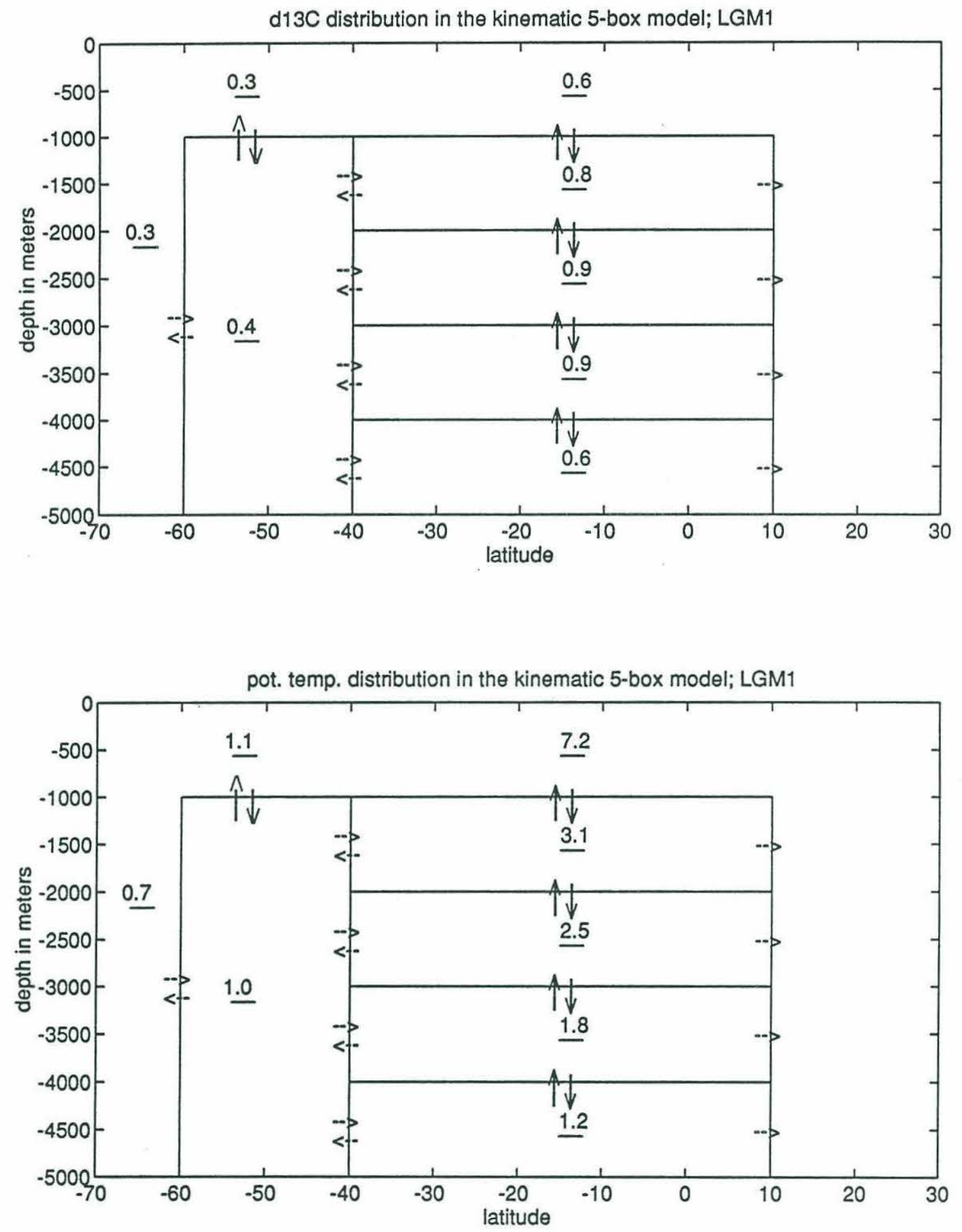

Figure V-B-20: $\delta^{13} \mathrm{C}(\% \circ)$ and potential temperature $\left({ }^{\circ} \mathrm{C}\right)$ distribution in the modern South Atlantic estimated by the kinematic 5-box model. Southernmost value corresponds to mean tracer value advected through Drake Passage. 


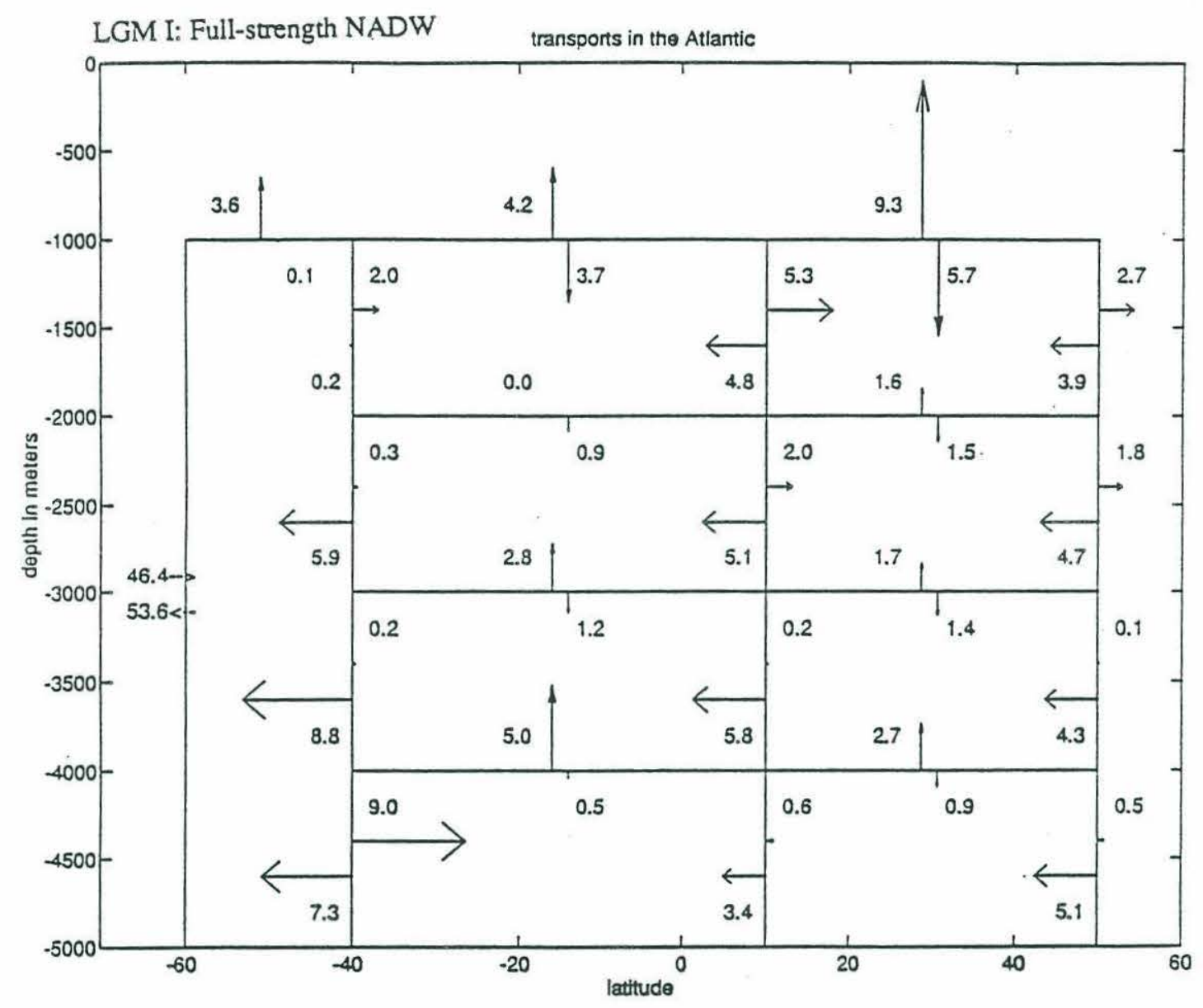

Figure V-B-21: Northward and southward zonally integrated transports (in Sv) in the LGM Atlantic (simulation I, full-strength NADW). Fluxes north of the equator estimated by dynamical box model (97 boxes). Fluxes south of the equator estimated by kinematic 5-box model (forced with fluxes across $10^{\circ} \mathrm{N}$ computed by dynamical box-model). Volume fluxes into and out of Atlantic sector of Circumpolar Current are in reality perpendicular to the meridional plan. The arrows corresponding to these fluxes are not scaled. 


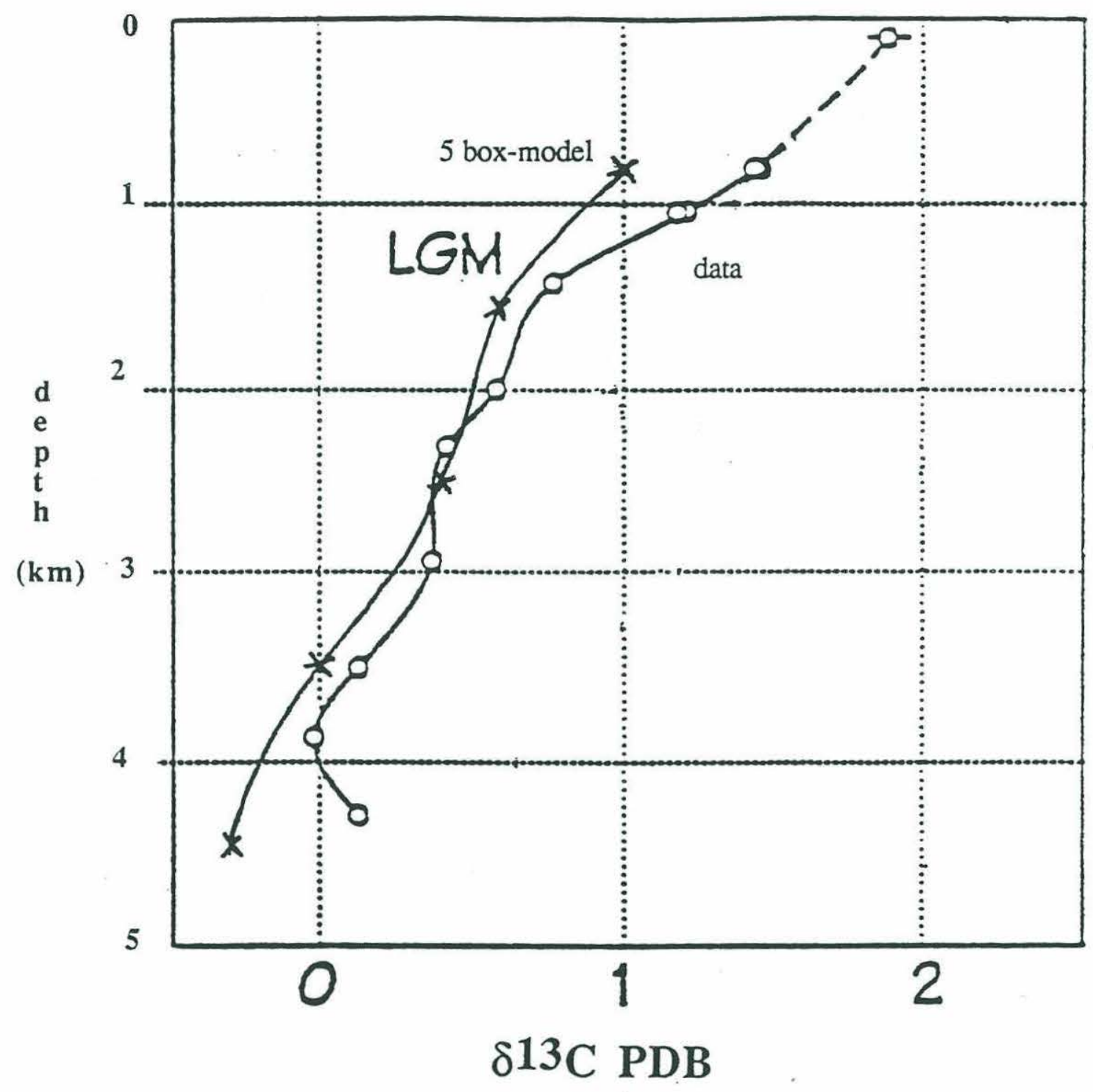

Figure V-B-22-a: Comparison of results of a 5-box model of the South Atlantic (LGM I, full-strength NADW) with a LGM vertical profile of $\delta^{13} \mathrm{C}$ at the Rio Grande Rise $\left(30^{\circ} \mathrm{S}\right)$. Data from Lohmann and Lohmann (1994). The top box model $\delta^{13} \mathrm{C}$ value is estimated as a boundary condition by the model, and is subjectively assigned to a depth of $800 \mathrm{~m}$. 


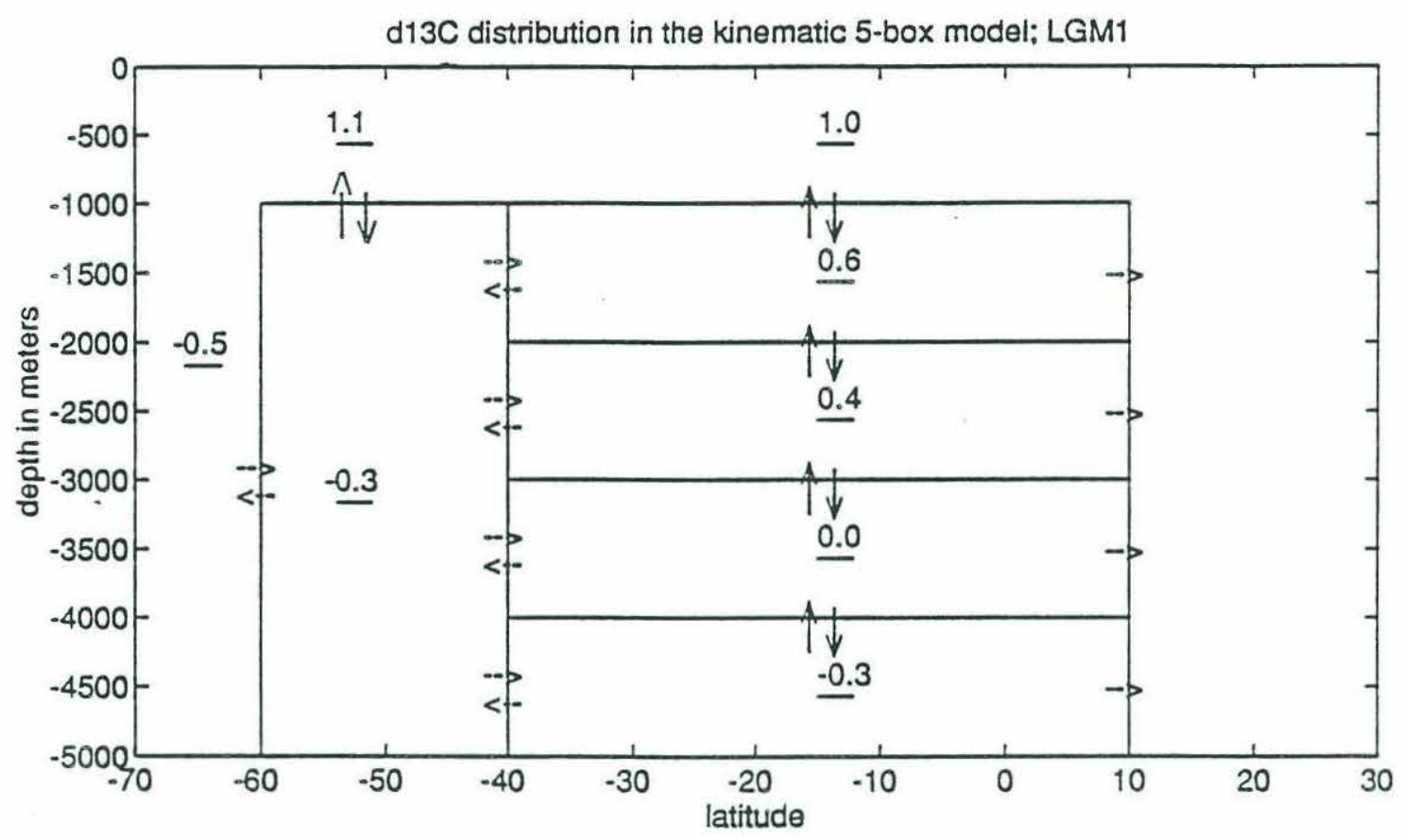

pot. temp. distribution in the kinematic 5-box model; LGM1

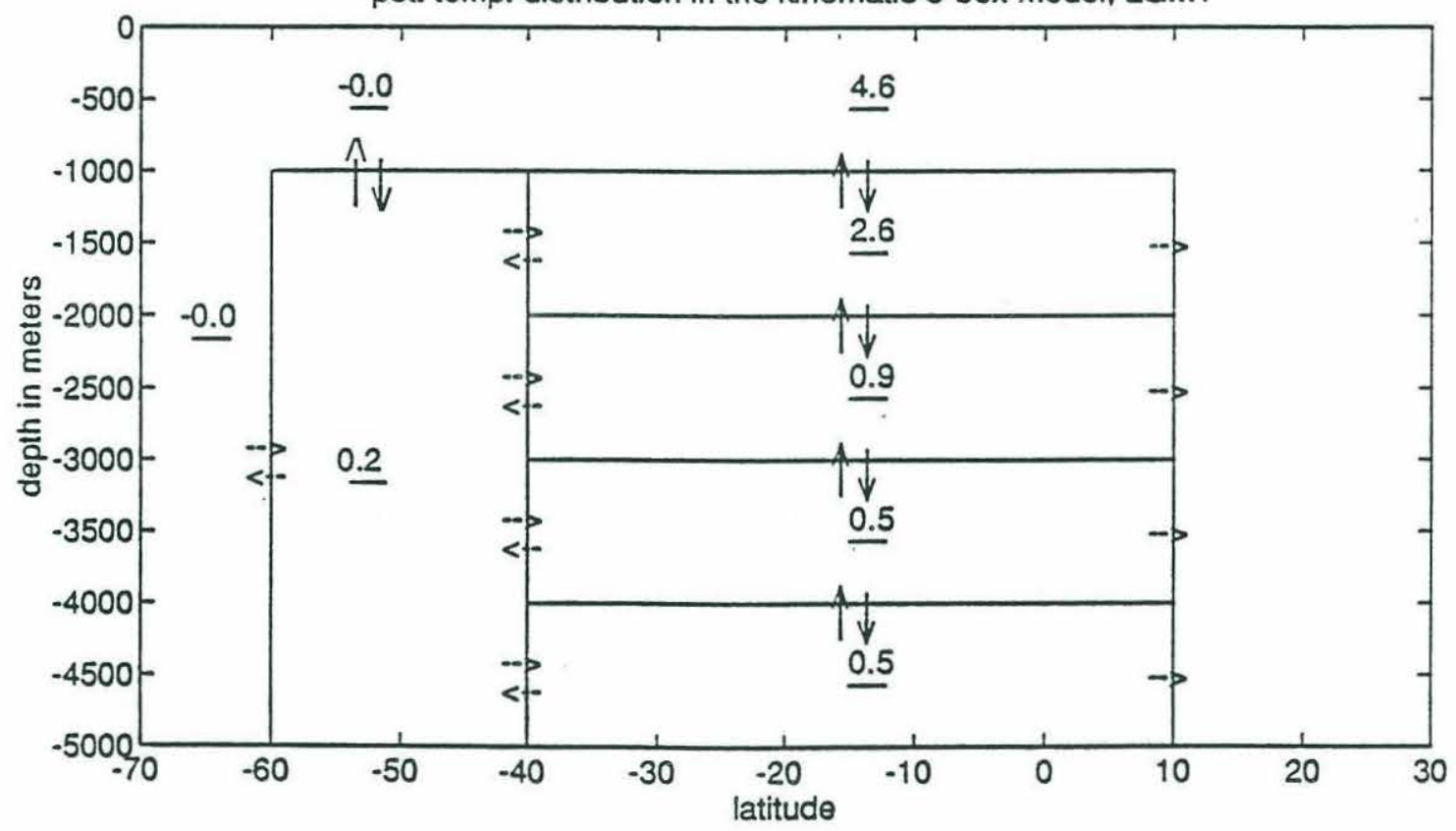

Figure V-B-22-b: Comparison of results of a 5-box model of the South Atlantic (LGM I, Full-strength NADW simulation) with a LGM vertical profile of $\mathrm{d}^{13} \mathrm{C}$ at the Rio Grande Rise ( $\left.30^{\circ} \mathrm{S}\right)$. Data from Lohmann and Lohmann (1994). 
Thus, as in the modern ocean case, a solution consistent with the available $\delta^{13} \mathrm{C}$ data could be found in the South Atlantic using the fluxes across $10^{\circ} \mathrm{N}$ computed from the North Atlantic simulation run [1].

\section{c/ LGM simulation of the South Atlantic with reduced Lower NADW} formation

This run is similar to the one described in the previous section except that the Northern boundary conditions are computed from the reduced Lower NADW flux simulation (run [2], see section V-B-3). The exchanges with the North Atlantic represent what happens in a scenario where the rate of formation of Lower NADW is reduced by $50 \%$ compared to its modern ocean value. Compared to the run described in the previous section, upwelling is reduced at every depth in the South Atlantic, and downwelling increases (Figure V-B-23). As a result, there is a net downwelling at depths of 1 and $2 \mathrm{~km}$. Compared to the previous run, the northward flux of water below $2 \mathrm{~km}$ depth across $40^{\circ} \mathrm{S}$ remains about the same, but the flux of deep water from the South Atlantic into the Southern Ocean below $2 \mathrm{~km}$ depth is reduced by about $7 \mathrm{~Sv}$ (compare Figure VB-21 with figure V-B-23).

The tracer distribution is almost identical to that of the previous run (compare Figure V-B-24 with Figure V-B-22), even though the flux of NADW has been reduced by $50 \%$. This similarity shows that tracer distribution and circulation can be partly decoupled. In this particular run, it seems that the source of ${ }^{13} \mathrm{C}$ rich water from the North Atlantic is compensated by a net downwelling of ${ }^{13} \mathrm{C}$ rich surface waters as well as a reduced leakage of NADW into the Circumpolar Current. The implications of this result are discussed in details in section V-C-4.

\section{Summary}

We have seen that two different circulations are consistent with the LGM $\delta^{13} \mathrm{C}$ and $\delta^{18} \mathrm{O}$ data. The first circulation is almost identical to the one we estimated for the modern ocean. The second one has a weaker DWBC and a weaker southward flux across $10^{\circ} \mathrm{N}$, which we interpret as 


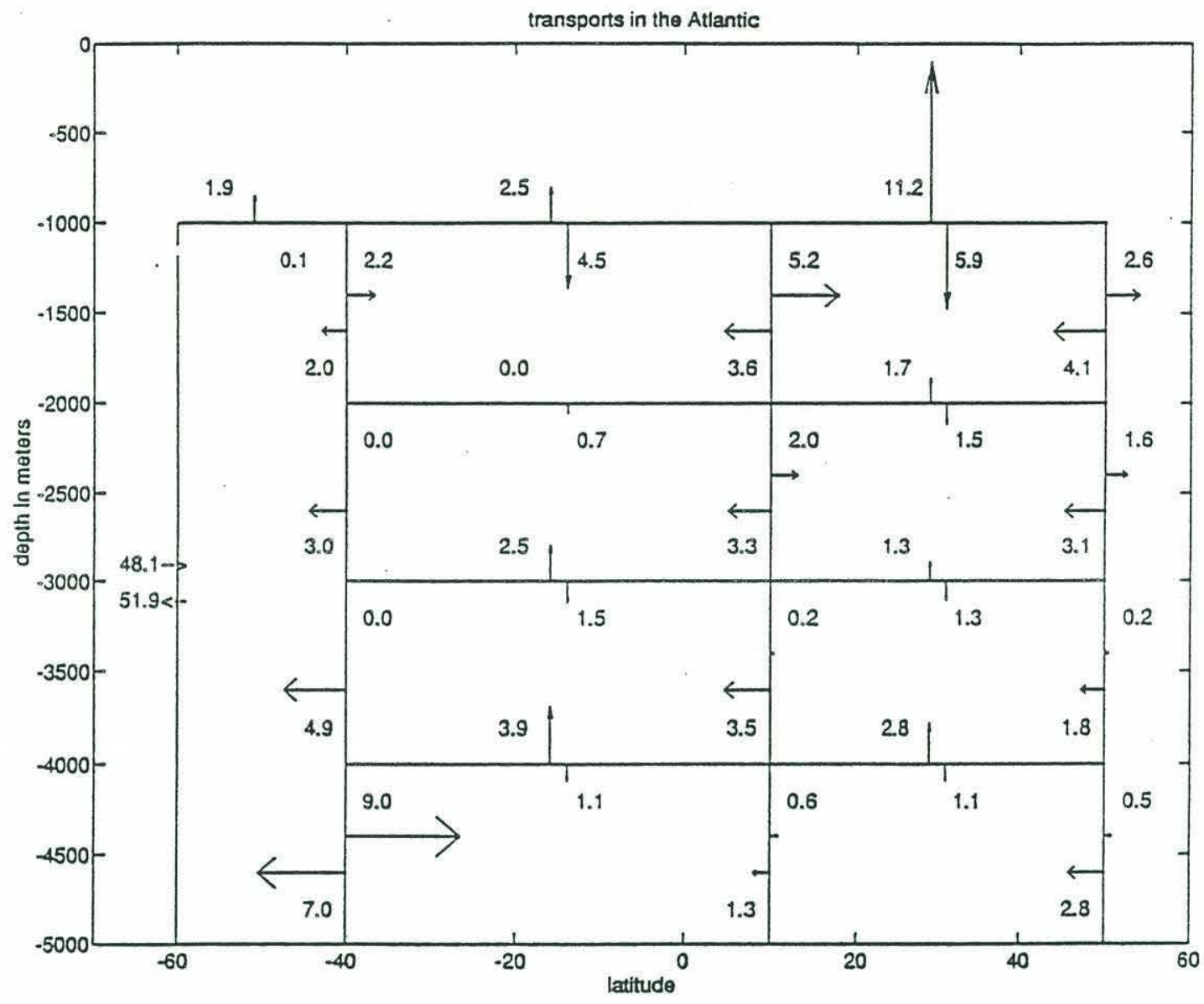

Figure V-B-23: Northward and southward zonally integrated transports (in Sv) in the LGM Atlantic (simulation II, reduced-strength NADW). Fluxes north of the equator estimated by dynamical box model (97 boxes). Fluxes south of the equator estimated by kinematic 5-box model (forced with fluxes across $10^{\circ} \mathrm{N}$ computed by dynamical box-model). Volume fluxes into and out of Atlantic sector of Circumpolar Current are in reality perpendicular to the meridional plan. The arrows corresponding to these fluxes are not scaled. 


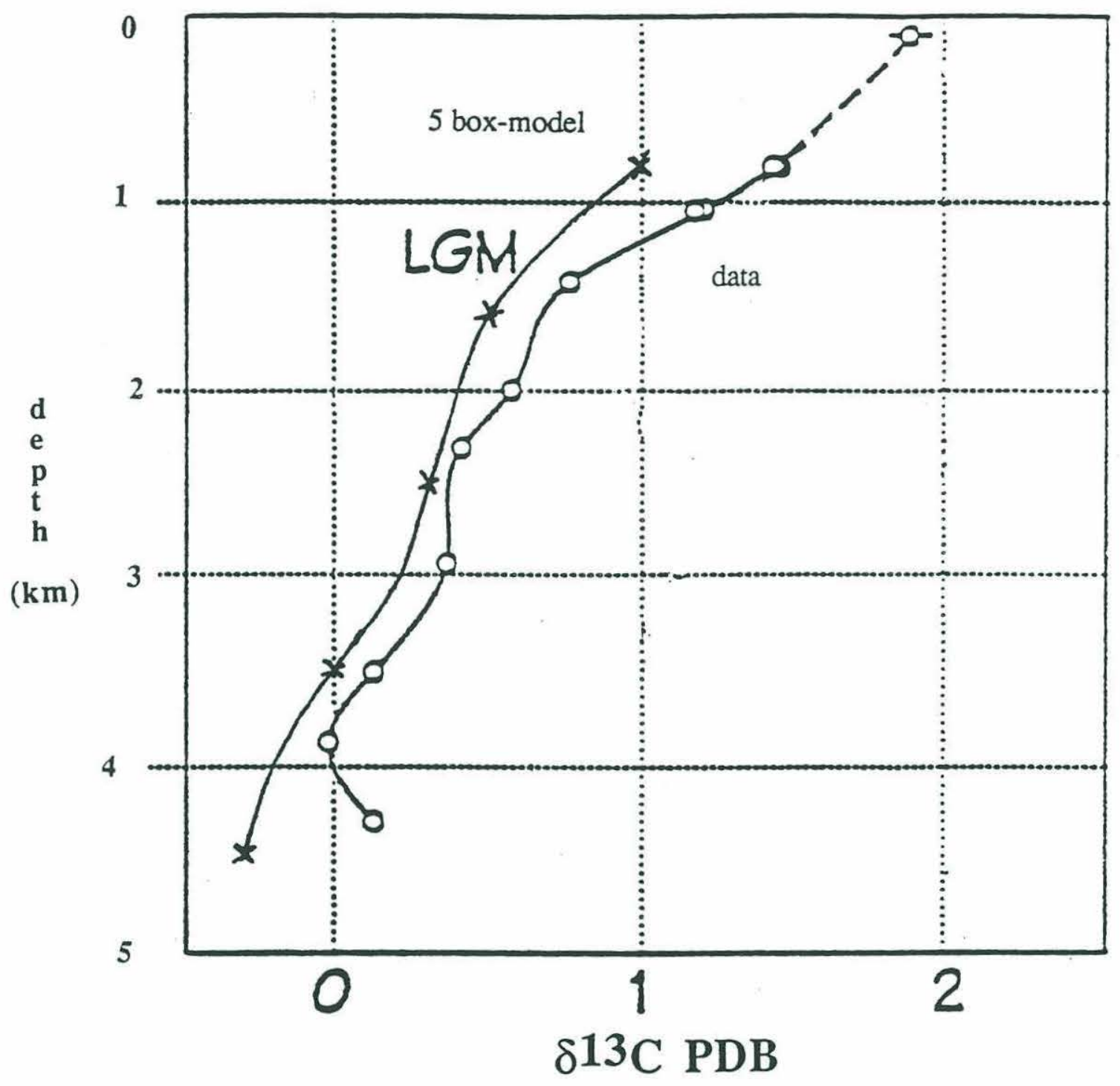

Figure V-B-24-a: Comparison of results of a 5-box model of the South Atlantic (LGM II, reduced-strength NADW) with a LGM vertical profile of $\delta^{13} \mathrm{C}$ at the Rio Grande Rise $\left(30^{\circ} \mathrm{S}\right)$. Data from Lohmann and Lohmann (1994). The top box model ${ }^{13} \mathrm{C}$ value is estimated as a boundary condition by the model, and is subjectively assigned to a depth of $800 \mathrm{~m}$. 

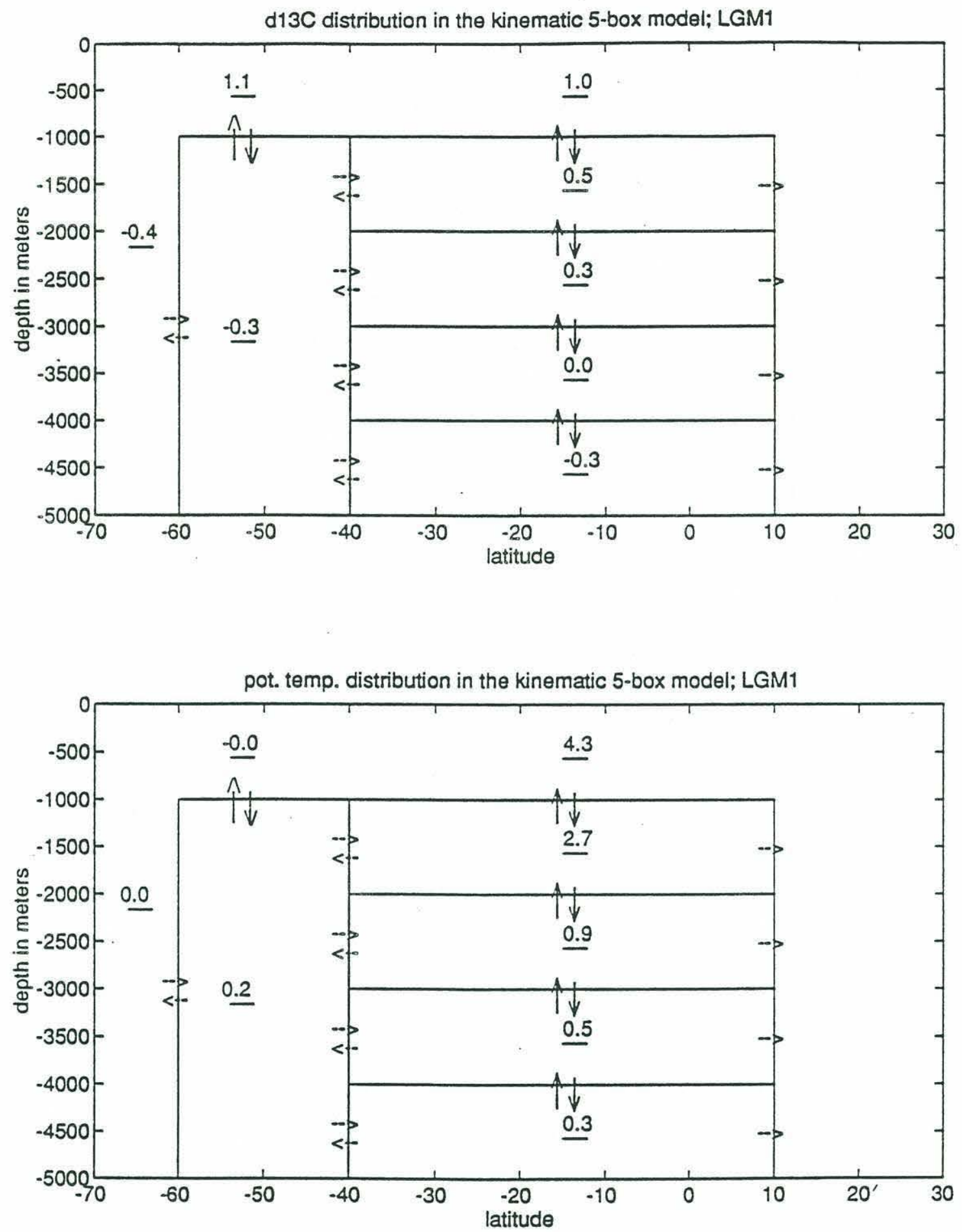

Figure V-B-24-b: Values of $\mathrm{d}^{13} \mathrm{C}$ in \%o (upper panel) and potential temperature in ${ }^{\circ} \mathrm{C}$ (lower panel) predicted by the kinematic 5-box model of the South Atlantic. LGM simulation II (reduced-strength NADW). 
being the consequence of a reduced rate of formation of Lower NADW. These two scenarios are also consistent with the LGM South Atlantic data in the context of a simple box model.

\section{C - Discussion}

We saw in section V-B-3 that it is possible to find at least one oceanic circulation compatible with paleo-data and realistic physics. This result is in itself important. Previous box model simulations did not include the thermal wind or the linear vorticity balances. The present model has a higher resolution which allows for the discretization of these important constraints. Moreover, unlike previous models (e.g., Keir, 1988), the data are not collapsed into only two or three values supposed to characterize the different water masses. The three-dimensional nature of the data set is represented. The fact that a reasonable solution can be found shows that the paleodata are compatible with known ocean physics, and that the water mass distribution they suggest is plausible.

At least two different ocean circulations can reproduce the observed distribution of $\delta^{13} \mathrm{C}$ and $\delta^{18} \mathrm{O}$ in the LGM Atlantic Ocean. One circulation scheme is similar to the modern one estimated in part A. It differs substantially from the now conventional interpretation of the Last Ice Age data (Boyle and Keigwin, 1987; Duplessy et al., 1988) where it has been claimed that a reduced rate of formation of Lower NADW is required. The second circulation scheme shows that a reduced production of Lower NADW is also compatible with the data. However, the tracer distributions obtained with the two different schemes are equally acceptable. The tracer data do not require a major change in the fluxes of deep water into the Atlantic. The model simulations suggest that the problem is less the determination of a circulation consistent with the paleo-tracer distribution than to find a way to narrow the apparent broad range of possible solutions.

There are formally an infinity of possible solutions. About $10 \%$ of the eigenvalues of the Hessian matrix (matrix of second partial derivatives of the objective function) almost vanish, which implies that the curvature of the objective function is very small along the associated eigenvectors (Gill et al., 1981). This small curvature means that the minimum of the objective function lies in flat valleys, and that there are an infinity of points which minimize almost equally 
well the objective function. It is argued in section V-C-2 that the integrated transport in the DWBC is quite independent from the tracer distribution in the Eastern Atlantic, where most of the paleodata come from, and thus that this transport can vary over a broad range of values without affecting the solution. The integrated volume flux in the DWBC probably corresponds to a vanishing value of the Hessian matrix. It is necessary to compute the eigenvectors to verify this presumption, which has not been done because of the size of the problem (the size of the Hessian matrix is $800 \times 800$, and it would have been necessary to find the eigenvector associated to the integrated transport in the DWBC among about 800 eigenvectors).

These model results, and their implications for the interpretation of paleo-data in terms of circulation changes are discussed in the following sections. Section 1 analyses how almost the same ocean circulation can be made consistent with both the modern and the LGM tracer distributions. Sections 2 and 3 explain why it is possible, in the models we use, to maintain a standing crop of tracer with different ocean circulations.

\section{1) Why is it possible to reproduce the LGM tracer distribution without changing much the modern ocean circulation?}

We saw in section V-B-2 that it is not necessary to make many changes in the present North Atlantic circulation in order to be consistent with the Ice Age ${ }^{13} \mathrm{C}$ data. Since tracer concentrations were significantly different during the LGM, fluxes of ${ }^{13} \mathrm{C}$ into and out of the North Atlantic did change. However, it is possible to find a tracer distribution consistent with the LGM data within the observational uncertainty, such that the ${ }^{13} \mathrm{C}$ fluxes balance in each box. The observational uncertainty is quite large, partly because of the lack of data in the Western Atlantic, in the upper ocean, and below $4 \mathrm{~km}$ depth in the Eastern basin (Chapter II). As more observations become available, the range of plausible distributions of $\delta^{13} \mathrm{C}$ should be narrowed, and larger circulation changes may be needed to accommodate the tracer data. But, so far there are not enough data to make these changes necessary.

Tracer fluxes into the Atlantic are set by the volume of deep water advected into the basin multiplied by preformed tracer concentrations. Thus, there are two ways to produce the more 
negative $\delta^{13} \mathrm{C}$ values in the Deep North Atlantic shown in Figure V-C-1: advecting less $\delta^{13} \mathrm{C}$-rich NADW (or more $\delta^{13} \mathrm{C}$-depleted $\mathrm{AABW}$ ), or advecting the same amount of NADW and AABW with more negative $\delta^{13} \mathrm{C}$ end-member values in $\mathrm{AABW}$. The latter mechanism is basically what happens in run [1]. In this simulation, low $\delta^{13} \mathrm{C}$ values $(-0.4 \%$ ) in the Southern Ocean box are advected northward into the lowest box of the South Atlantic basin and then into the deep layers of the North Atlantic. These values are sufficiently negative so that it is not necessary to reduce the flux of NADW across $50^{\circ} \mathrm{N}$ in order to obtain low $\delta^{13} \mathrm{C}$ values in the deep North Atlantic (Figure $\mathrm{V}-\mathrm{B}-21)$. Therefore, the model simulations suggest that the $\delta^{13} \mathrm{C}$ distribution in the glacial Atlantic can be explained by lower $\delta^{13} \mathrm{C}$ end-member values in $\mathrm{AABW}$ and that the circulation need not be significantly different from the modern one.

\section{2) Why is it possible to find several oceanic circulations compatible with the LGM $\delta^{13} \mathrm{C}$ distribution?}

The first difficulty with the steady state interpretation of $\delta^{13} \mathrm{C}$ data, or any other passive tracer data, in terms of rate of circulation is that the ${ }^{13} \mathrm{C}$ distribution is not affected if one multiplies all the mass fluxes by an arbitrary constant. All that is required to maintain a given passive tracer distribution in a basin is that the flux divergence vanishes, and one cannot infer the tracer flux itself unless there is some externally prescribed rate setting number available. Geostrophy provides such a rate setting number in the modern ocean, but not in the glacial ocean, because the gradients of density are unknown.

It has been suggested, however, that one could at least estimate the rate of production of NADW relative to AABW from such tracers (Boyle and Keigwin, 1982). This idea can be illustrated by a very simple 1-box model of the Deep Atlantic (see Figure V-C-2). 


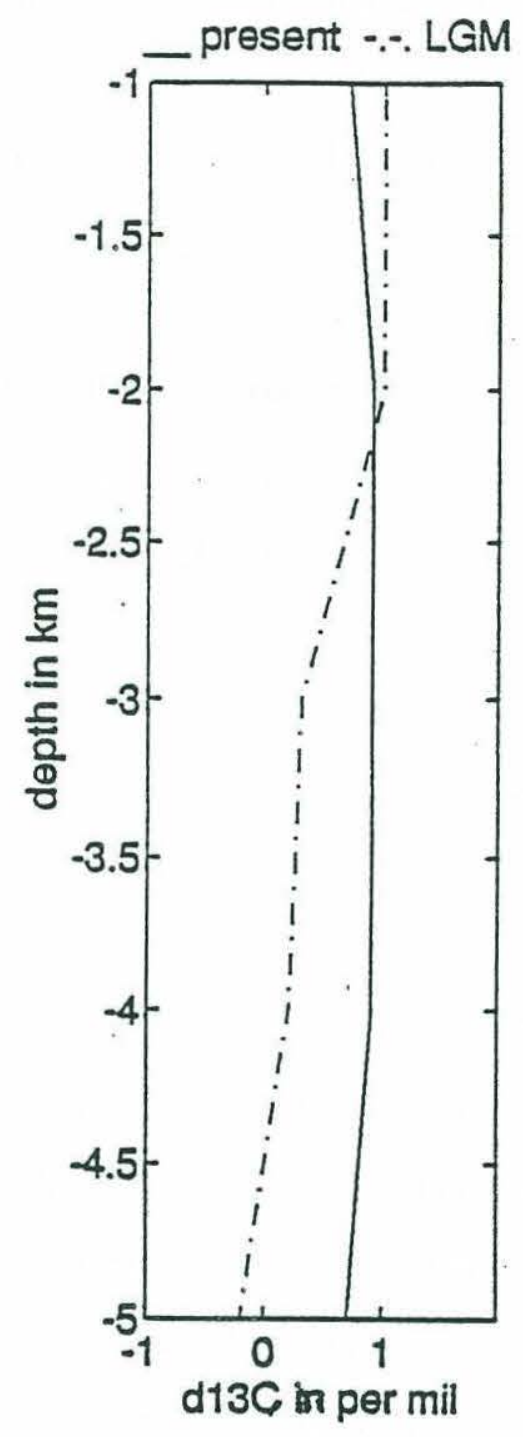

Figure V-C-1: Vertical profiles of $8^{13} \mathrm{C}$ at $20^{\circ} \mathrm{W}, 20^{\circ} \mathrm{N}$ estimated by the dynamical boxmodel. Full line represents the modern ocean profile (calculated from phosphate values). Dot-dash line represents the profile calculated in the LGM I simulation (full-strength NADW). The observation that intermediate waters are enriched in $\delta^{13} \mathrm{C}$ and deep waters are depleted in $\delta^{13} \mathrm{C}$ in the North Atlantic during glacial times, compared to modern times, is reproduced in the model simulations. 


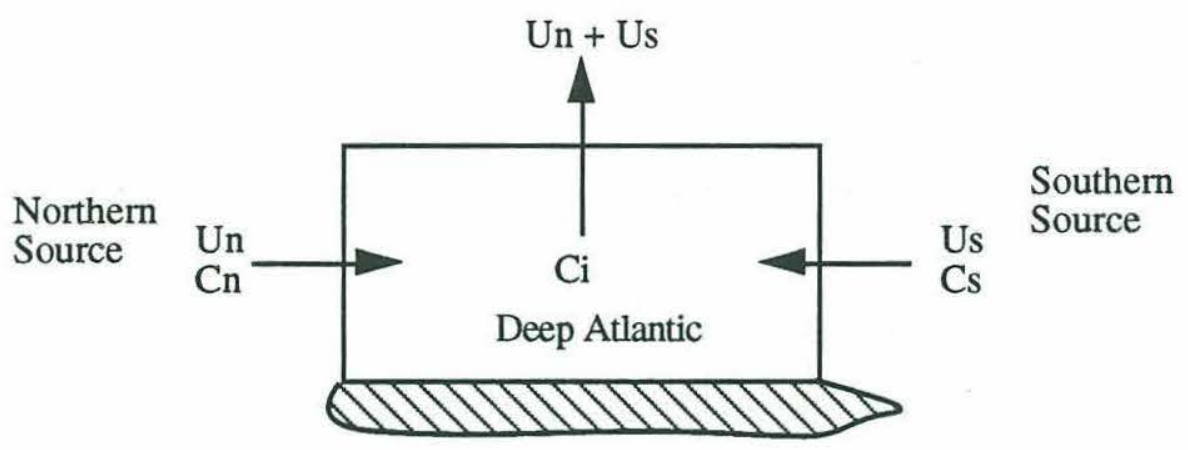

Figure V-C-2: 1-box model of the deep Atlantic with two sources of tracer

Tracer conservation in the interior of the box can be written in terms of contributions of Northern $\left(U_{n}\right)$ and Southern $\left(U_{S}\right)$ sources:

$$
\text { (1) } \frac{U_{s}}{U_{n}}=\frac{C_{n}-C_{i}}{C_{i}-C_{s}}
$$

$\mathrm{C}_{\mathrm{n}}$ and $\mathrm{C}_{\mathrm{S}}$ represent the northern and southern end-members and $\mathrm{C}_{\mathrm{i}}$ a typical concentration in the middle of the basin. Note that $U_{n}$ and $U_{S}$ can be multiplied by any arbitrary constant without affecting the tracer distribution. Yet, in this very simple model, the ratio of the flux of NADW over the flux of $\mathrm{AABW}$ can be directly determined from ${ }^{13} \mathrm{C}$ data.

However, such a simple interpretation of the data is not possible in more realistic models of the ocean. Passive tracers constrain the local mass balance but not the global one, and, in models with more than one box, it is usually not possible to determine the ratio Us/Un from the tracer field alone. This problem can be illustrated by a 2-box model, which can be thought of as a model of the North Atlantic, that treats the DWBC and the interior of the ocean separately (Figure V-C3). 


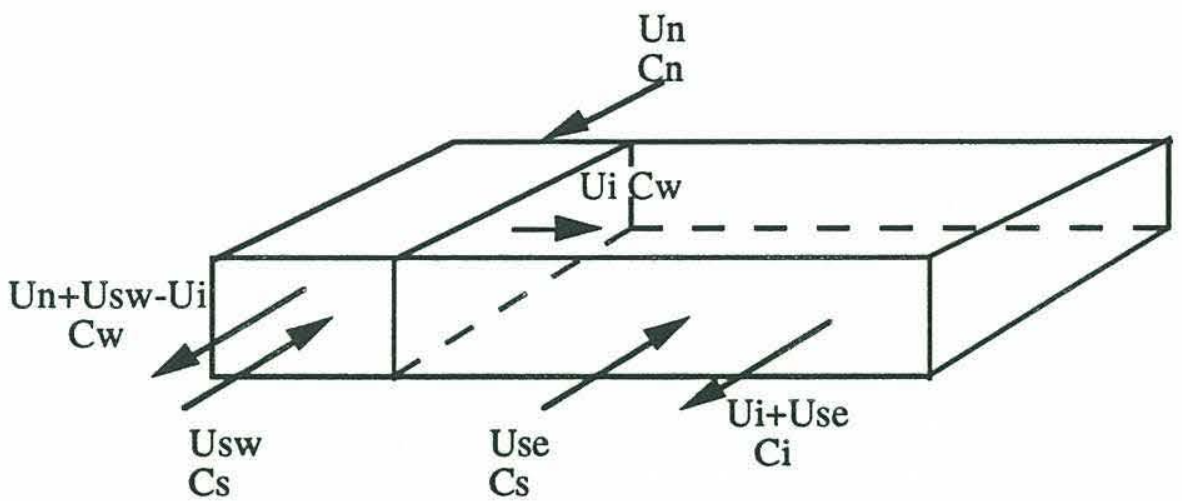

Figure V-C-3: 2-box model of the North Atlantic. The interior of the North Atlantic is fed from a southern source and a recirculation from the western boundary. There is no interior flow through the northern boundary. $\mathrm{U}_{\mathrm{Sw}}$ and $\mathrm{U}_{\mathrm{Sw}}+\mathrm{U}_{\mathrm{n}}-\mathrm{U}_{\mathrm{i}}$ represent respectively the northward flow of AABW (west of the Mid-Atlantic Ridge) and the southward flow of NADW across the southern boundary of the North Atlantic. Use represents the northward flow of AABW east of the Mid-Atlantic Ridge. It is assumed that $U_{S W}$ and $U_{\text {Se }}$ advect water masses with the same tracer composition.

$\mathrm{C}_{\mathrm{S}}$ and $\mathrm{C}_{\mathrm{n}}$ are the southern and northern end-members. $\mathrm{C}_{\mathrm{W}}$ and $\mathrm{C}_{\mathrm{i}}$ are the tracer concentrations in the DWBC and in the interior of the Atlantic. The tracer budgets for the two boxes can be written as:

$$
\frac{\mathrm{U}_{\mathrm{sw}}}{\mathrm{U}_{\mathrm{n}}}=\frac{\mathrm{C}_{\mathrm{n}}-\mathrm{C}_{\mathrm{w}}}{\mathrm{C}_{\mathrm{w}}-\mathrm{C}_{\mathrm{s}}}
$$

and

$$
\frac{U_{s e}}{U_{i}}=\frac{C_{w}-C_{i}}{C_{i}-C_{s}}
$$

combining the two expressions, we obtain:

$$
\text { (2) } \frac{U_{s}}{U_{n}}=\frac{U_{s w}+U_{s e}}{U_{n}}=\frac{C_{n}-C_{w}}{C_{w}-C_{s}}+\frac{U_{i}}{U_{n}} \times \frac{C_{w}-C_{i}}{C_{i}-C_{s}}
$$

Thus, in this 2-box model, tracer data alone do not suffice to constrain the relative rates of formation of NADW and AABW. Some direct information on the rate of ventilation of the interior of the ocean is needed $\left(\mathrm{U}_{\mathrm{i}} / \mathrm{U}_{\mathrm{n}}\right.$ for instance). In the modern ocean, the thermal wind balance provides such direct information on the ventilation rate of the ocean; the gradients of density are not available in the glacial ocean, and the thermal wind balance provides little information there. 
Relation (2) does not reduce to relation (1), unless $C_{W}-C_{i}$ is negligible compared to $C_{i}-C_{S}$, which in turns implies that $U_{s e}$ is negligible compared to $U_{i}$, i.e. the Eastern Atlantic is mostly ventilated by waters recirculating from the DWBC with very little contribution from the Southern Ocean. There is evidence in the modern ocean for significant fluxes of AABW into the Eastern Atlantic (Warren, 1981; McCartney et al., 1991) and for East-West phosphate gradients (difference of about $0.3 \mu \mathrm{mol} / 1$ at $24^{\circ} \mathrm{N}$, see Figure V-C-4), non-negligible when compared to the difference between the Northern and the Southern end-members (about $1 \mu \mathrm{mol} / \mathrm{l}$, see GEOSECS Atlantic section, 1980). For the deep glacial ocean, there are not enough data points in the Western Atlantic to conclude, but one may expect east-west gradients to have also existed during the Last Ice Age. Thus, neglecting the East-West gradients is probably not justified and, not too surprisingly, one cannot use relation (1) as a reasonable approximation of the tracer budget in the Atlantic.

The 2-box model, though probably not very realistic itself, provides some insight on the results of the dynamical box model of the North Atlantic. It suggests that the $\delta^{13} \mathrm{C}$ distribution in the Eastern Atlantic (where most of the paleo-data come from) constrains the ventilation rate of the interior of the Atlantic, but is somewhat decoupled from the flux of NADW represented by the DWBC. An extreme case (though not unreasonable if the 2-box model is viewed as a model of the North Atlantic) occurs when the rate of formation of NADW $\left(U_{n}\right)$ is large compared to the northward flow of $\mathrm{AABW}$ in the western Atlantic $\left(\mathrm{U}_{\mathrm{Sw}}\right)$; then the tracer concentration in the $\operatorname{DWBC}\left(\mathrm{C}_{\mathrm{W}}\right)$ is almost equal to that of the northern end-member $\left(\mathrm{C}_{\mathrm{n}}\right)$, and

$$
\frac{\mathrm{U}_{\mathrm{se}}}{\mathrm{U}_{\mathrm{i}}} \approx \frac{\mathrm{C}_{\mathrm{n}}-\mathrm{C}_{\mathrm{i}}}{\mathrm{C}_{\mathrm{i}}-\mathrm{C}_{\mathrm{s}}}
$$

This relation is identical to relation (1) obtained with the 1-box model, except that the zonally integrated transports have been replaced by the transports ventilating the Eastern Atlantic. In this 2-box model, as long as $\mathrm{C}_{\mathrm{n}}$ and $\mathrm{C}_{\mathrm{S}}$ are fixed, $\mathrm{C}_{\mathrm{i}}$ only constrains $\mathrm{U}_{\mathrm{Se}} / \mathrm{U}_{\mathrm{i}}$, but does not constrain $\mathrm{U}_{\mathrm{n}}$, the rate of formation of NADW. In other words, Eastern Atlantic data only constrain the ventilation of the eastern basin, but not the flux of NADW in the DWBC.

This decoupling between the DWBC and the ventilation of the interior of the ocean can be seen in the dynamical box model as well, by comparing run [1] with run [2]. The strength of the 


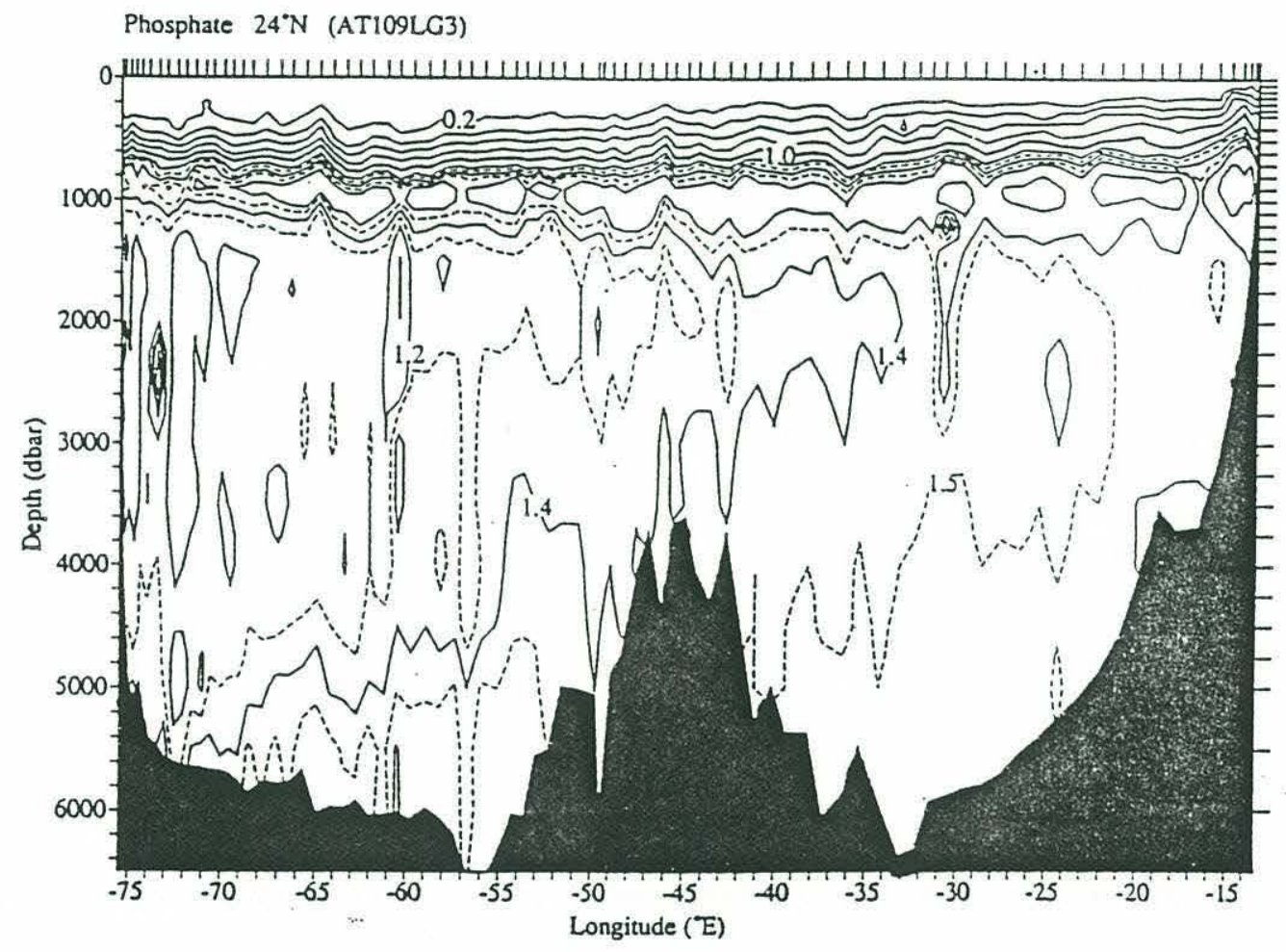

Figure V-C-4: Zonal section of phosphate in the North Atlantic observed during the early 1980's. From Roemmich and Wunsch (1985). This section shows significant EastWest gradients in the tracer distribution at mid-latitudes. 
DWBC is about $50 \%$ smaller in run [2]. Yet, there is almost no difference in the estimated interior circulation and tracer distribution (compare Figure V-B-8 with Figure V-B-14). The main adjustments take place in the DWBC, and hardly affect the interior: there is simply a smaller flux of water in the DWBC boxes in run [2]. This result is also true for ${ }^{13} \mathrm{C}$. Figures V-C-5 and V-C6 show that the flux of $\delta^{13} \mathrm{C}$ (computed by multiplying the transports by the average $\delta^{13} \mathrm{C}$ value over each side of the boxes) in the DWBC is significantly smaller in run [2] but that the fluxes in the interior are the same. The fluxes from the DWBC into the interior are slightly different, but these differences are small compared to those in the DWBC.

It may be possible to infer the flux of NADW relative to AABW from data close to the DWBC. Indeed, close to the western boundary the north-south differences of $\delta^{13} \mathrm{C}$ are larger in the reduced production of Lower NADW scenario than in the full-strength NADW scenario, by about $0.1 \%$. This difference is probably too small to be detectable in the LGM North Atlantic data. But, extrapolated over the whole Atlantic Ocean, it would translate into a detectable 0.2 or $0.3 \%$ gradient increase. However, it is not clear how this signal could be distinguished from changes in the end-members composition which seem to have occurred in the past (the preformed $\delta^{13} \mathrm{C}$ of Southern Ocean waters seems to have been more negative during the LGM, and the North-South differences were larger everywhere, not only in the DWBC, see Chapter II).

Note that there is no dynamical reason why the recirculation into the interior should be proportional to the strength of the DWBC, or to the rate of formation of NADW. In the StommelArons model (Stommel and Arons, 1960a\&b), for instance, the strength of the interior flow depends on the strength of the upwelling, assumed to be uniform, which in turn, by continuity, is linked to the difference between sources and sinks of deep water. In this dynamical model, one can have a strong DWBC but a weak interior flow if the sink is almost as large as the source. In the North Atlantic, the source is the amount of NADW being produced, and the sink is the amount of water leaving the basin across the equator. Similarly, the main difference between the LGM runs [1] and [2] is the strength of the DWBC. The mass exchanges between the DWBC and the interior are almost identical. Thus, in the context of the linear dynamics of the inverse model, it is possible to change the strength of the DWBC without affecting much the exchanges with the interior of the ocean. 

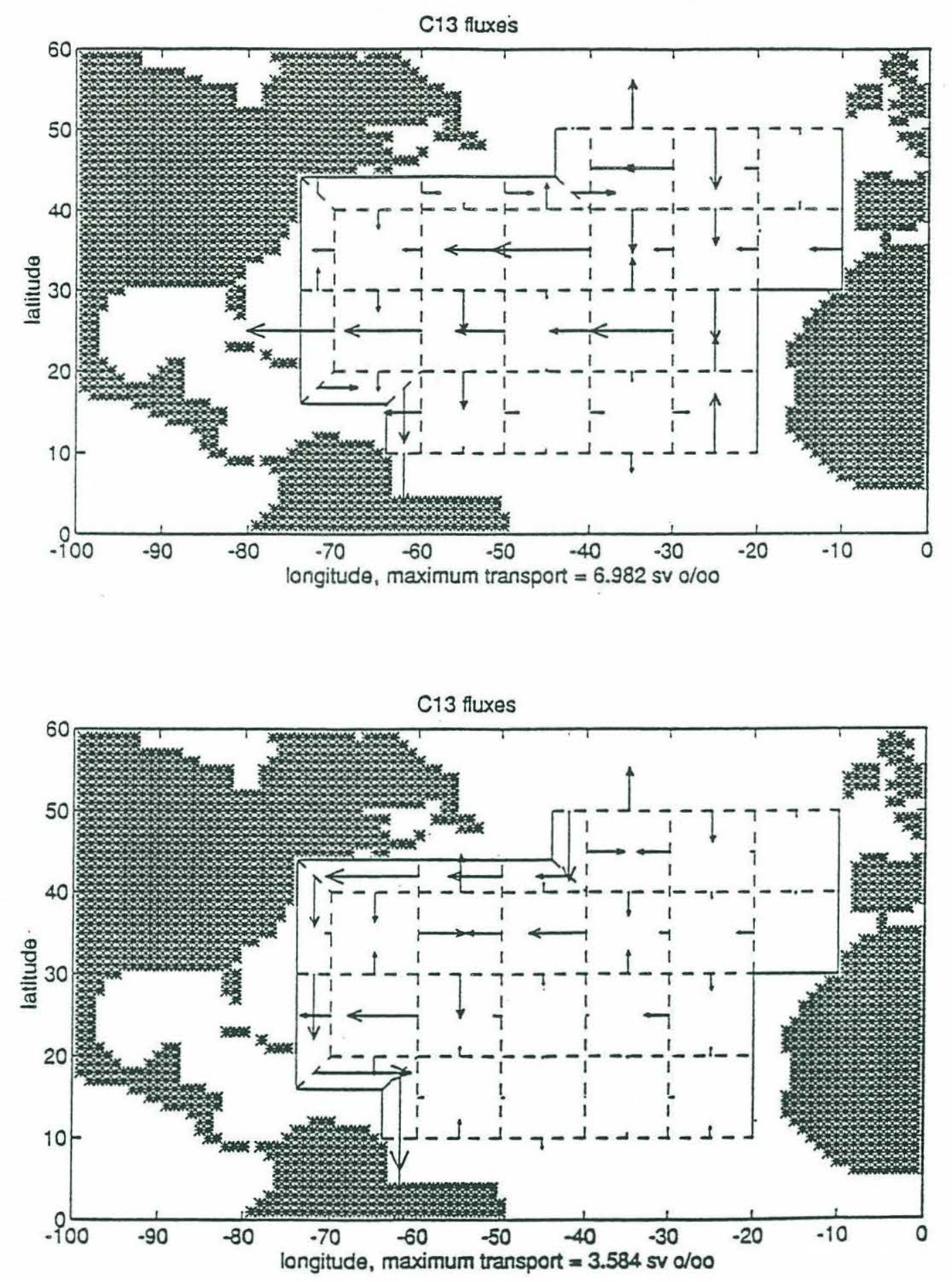

Figure V-C-5-a: Horizontal fluxes of $\delta^{13} \mathrm{C}$ (defined as the product of volume transports and $\delta^{13} \mathrm{C}$ values, and expressed in Sv.\%o), in the LGM simulation I (full-strength NADW). The flux associated with the longest arrow is indicated below each panel. Upper panel: vertically integrated flux between 1 and $2 \mathrm{~km}$ depth. Lower panel: vertically integrated flux between 2 and $3 \mathrm{~km}$ depth. 

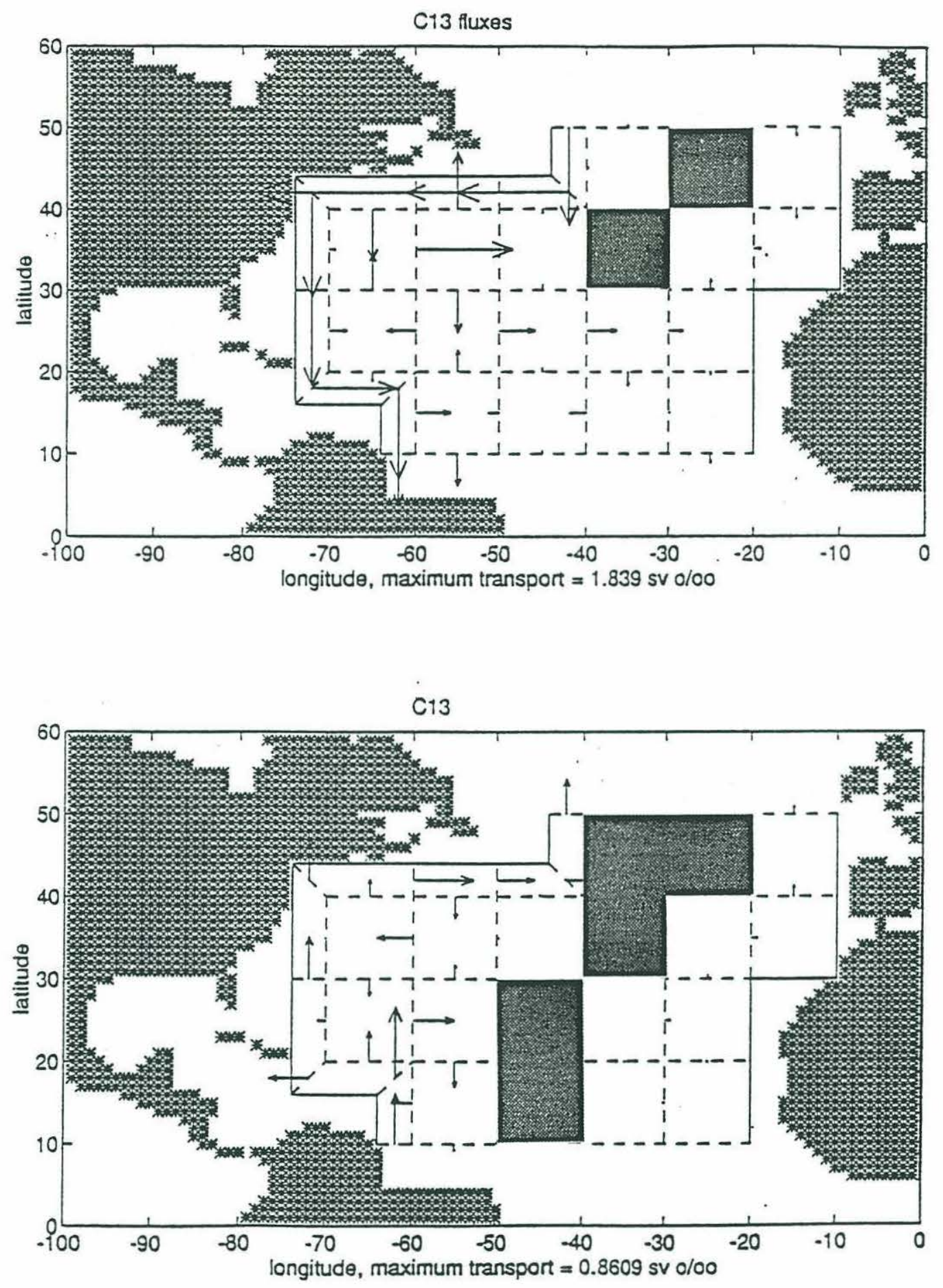

Figure V-C-5-b: Horizontal fluxes of $\delta^{13} \mathrm{C}$ (defined as the product of volume transports and $\delta^{13} \mathrm{C}$ values, and expressed in Sv.\%o), in the LGM simulation I (full-strength NADW). The flux associated with the longest arrow is indicated below each panel. Upper panel: vertically integrated flux between 3 and $4 \mathrm{~km}$ depth. Lower panel: vertically integrated flux between 4 and $5 \mathrm{~km}$ depth. 

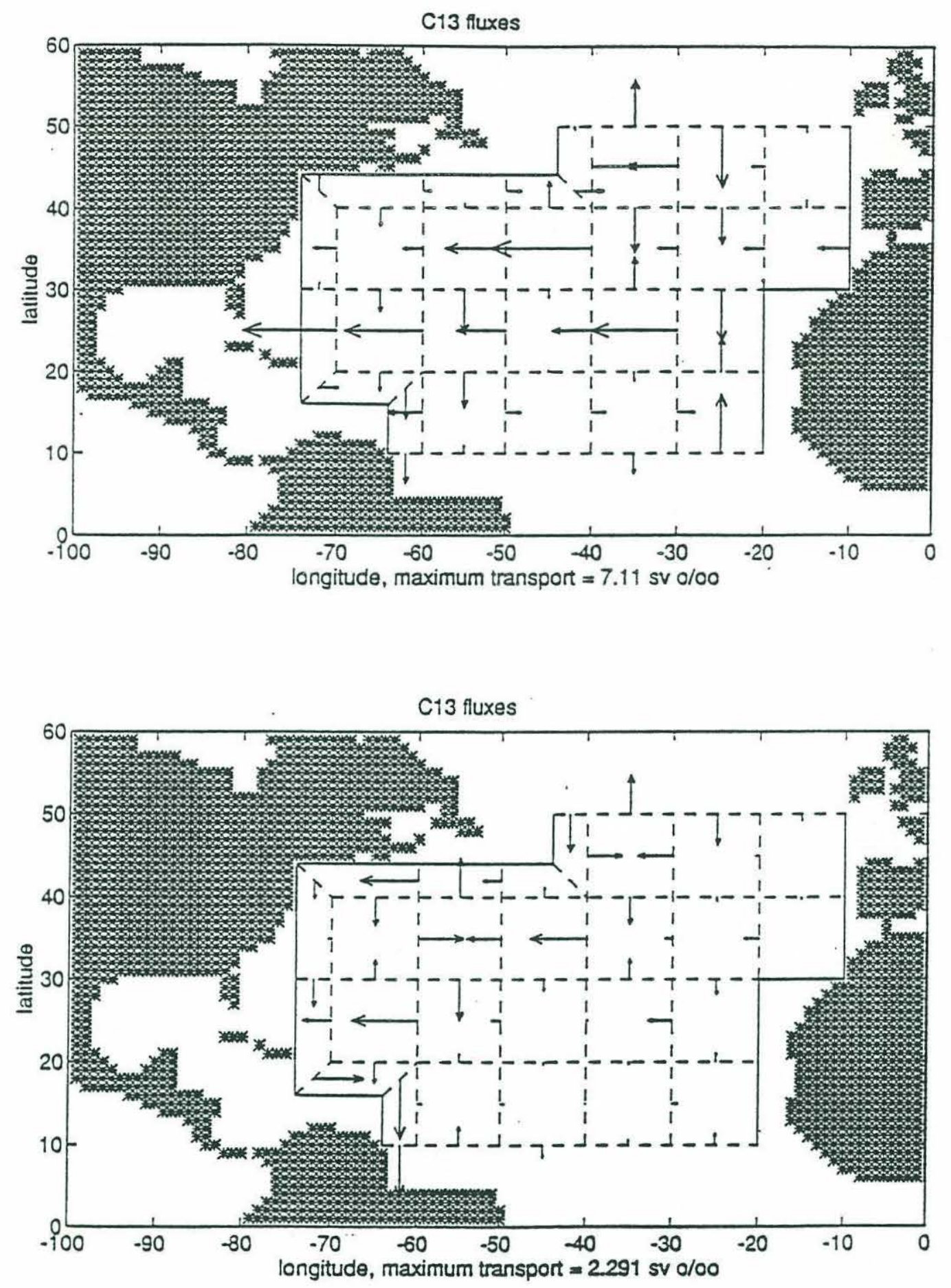

Figure V-C-6-a: Horizontal fluxes of $\delta^{13} \mathrm{C}$ (defined as the product of volume transports and $\delta^{13} \mathrm{C}$ values, and expressed in Sv.\%o), in the LGM simulation II (reduced-strength NADW). The flux associated with the longest arrow is indicated below each panel. Upper panel: vertically integrated flux between 1 and $2 \mathrm{~km}$ depth. Lower panel: vertically integrated flux between 2 and $3 \mathrm{~km}$ depth. 

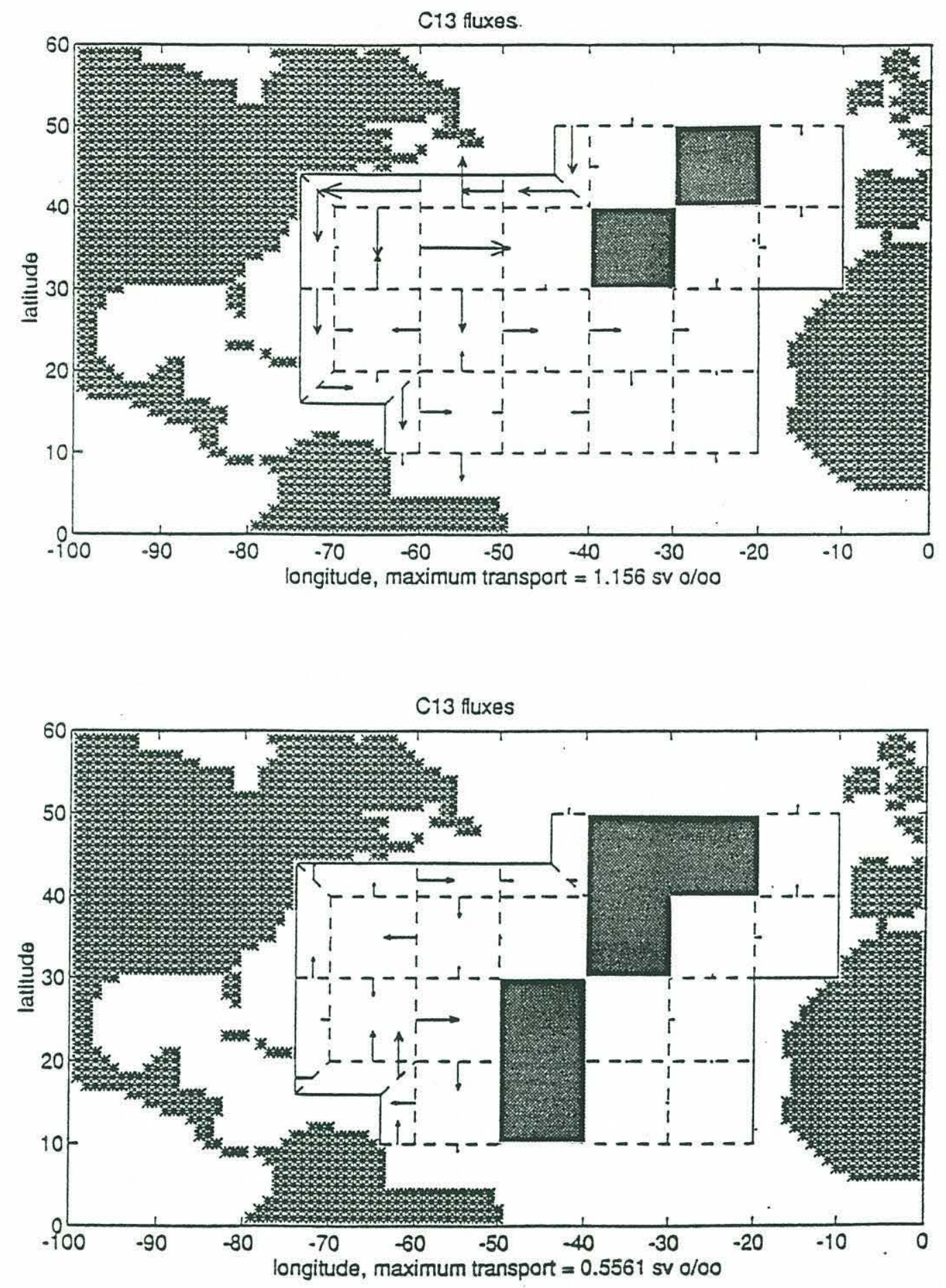

Figure V-C-6-b: Horizontal fluxes of $\delta^{13} \mathrm{C}$ (defined as the product of volume transports and $\delta^{13} \mathrm{C}$ values, and expressed in Sv.\%o), in the LGM simulation II (reduced-strength NADW). The flux associated with the longest arrow is indicated below each panel. Upper panel: vertically integrated flux between 3 and $4 \mathrm{~km}$ depth. Lower panel: vertically integrated flux between 4 and $5 \mathrm{~km}$ depth. 
Since the recirculation into the interior of the Atlantic need not be proportional to the strength of the DWBC, one can imagine an extreme case where there is strong formation of NADW but most of the NADW is transported by the DWBC to the Pacific Ocean, where it upwells. In this case, there would be a small recirculation of NADW into the interior even though NADW would be produced in large amounts. Thus, potentially large changes in the water mass distribution in the Eastern Atlantic could be produced by small changes in the recirculation of water from the DWBC, and negligible changes in the fluxes of NADW.

\section{3) Other sources of ambiguity on the interpretation of tracer distribution in terms of circulation}

The fundamental reasons why several circulations are consistent with the paleo-data in the model simulations presented in part $B$ are that passive tracers such as $\delta^{13} \mathrm{C}$ do not suffice to determine the absolute fluxes of water, and that they constrain the relative fluxes only locally. However, there are other factors which add to the ambiguity of the interpretation of the $\delta^{13} \mathrm{C}$ data in terms of circulation. These factors are less fundamental in the sense that they may be overcome as more data become available or they may be specific to the models we use, but they nonetheless represent serious obstacles.

\section{a/ Problems due to the lack of data or noise in the data}

Benthic foraminifera are not always very good "chemists" and record deep ocean geochemistry with some imprecision. Moreover, records are perturbed by fluxes of organic carbon in high productivity regions and by bioturbation in low sedimentation rate cores (see chapter II). Because it is difficult to quantify the effect of these processes, most paleoceanographic data are published without uncertainty estimates. However, in order to use the data quantitatively, one must estimate the uncertainties. For $\delta^{13} \mathrm{C}$, a rough uncertainty of $0.2 \%$ on average is reasonable, if somewhat arbitrary (see chapter II). $\delta^{13} \mathrm{C}$ values in the LGM deep Atlantic range from less than $-0.5 \%$ to more than $1 \%$ so the large scale signal is quite robust. Thus there is no 
doubt that the observed $\delta^{13} \mathrm{C}$ changes are real. There are still large areas such as the Western Atlantic, however, for which there are not many data. This, combined with the error bars, results in uncertainty in the inferred circulation. The principle of the propagation of uncertainties from tracer to circulation can be illustrated by the 1-box model depicted in Figure V-C-2. The ratio of the flux of water formed in the Southern Ocean Water over the flux of water formed in the North Atlantic is:

$$
\frac{\mathrm{Us}}{\mathrm{Un}}=\frac{\mathrm{Cn}-\mathrm{Ci}}{\mathrm{Ci}-\mathrm{Cs}}
$$

For typical $L G M$ values of $C_{n}=1 \%$, $C_{S}=-0.4 \%$ and $C_{i}=0.3 \%$, we obtain $U_{S} / U_{n}=1$; however, if $\mathrm{C}_{\mathrm{i}}$ is taken to be $0.5 \%$ instead of $0.3 \%$, $\mathrm{U}_{\mathrm{S}} / \mathrm{U}_{\mathrm{n}}=5 / 9 \approx 0.5$. The estimate of the ratio of the flux of water formed in the Southern Ocean Water over the flux of water formed in the North Atlantic can vary by a factor of two depending on a range of plausible values chosen for the interior concentration. Note that a $0.2 \%$ shift is within the data uncertainty. Small modifications of the tracer concentrations can result in very different estimates of the circulation, because the relationship between tracer and transport is nonlinear. Note that if the range of uncertainty in the observed data were more precisely known, it would be possible to estimate formally the range of possible circulations consistent with the tracer data.

Another difficulty arises because of the lack of reliable data in the South Atlantic. Different data sets suggest somewhat contradictory vertical profiles. We have seen that the boundary conditions at the southern edge of the model play an important role by setting the fluxes of tracer into the interior of the Atlantic. These fluxes appear to be poorly constrained by the available South Atlantic data even if one simulates the South Atlantic with a model with few degrees of freedom such as a 5-box model. Obtaining more and better data in the South Atlantic and in the Circumpolar Current should be a priority.

\section{b/ The ocean may not be in steady state balance}

An issue that is not addressed in the model simulations is that the circulation may not be in steady state at all. Climate has been fairly stable over the past 10000 years and it makes sense to model the pre-industrial oceanic circulation as a steady state process. However, there is good 
evidence that climate was much less stable during the LGM (Johnsen et al., 1992). Introducing non-stationary terms in the model would create more degrees of freedom and even smaller modifications of the modern ocean circulation would be necessary to accommodate the paleodata.

\section{c/ Model simplifications}

We have introduced a few simplifications in the model which could artificially add to the ambiguity of the problem of estimating the circulation from paleo-data.

For instance, the Indian and Pacific oceans, as well as the upper layers of the ocean, are not explicitly taken into account. The exchanges between the Atlantic and these basins are treated as variables to be estimated by the model from Atlantic data. Thus, the model runs are not constrained by data located in the Indian and Pacific oceans, or in the upper layers of the ocean. However, there are few data points there and it would be surprising if including them would significantly alter the various circulation schemes. The circulation and tracer field in these regions can probably be adjusted as in the model of the South Atlantic by changing the upwelling rate, for example. Moreover, there are some discrepancies between $\delta^{13} \mathrm{C}$ and Cadmium data in the Indian and Pacific Oceans (Boyle, 1992; see chapter II) which have not been sorted out yet.

Other neglected processes are the formation of Deep Water and the interaction between ocean and atmosphere. The atmospheric composition is quite well known for the LGM from ice core data, and would presumably put some constraints on the exchange of ${ }^{13} \mathrm{C}$ between the atmosphere and the ocean during formation of deep waters, and thus help to better define the

preformed values of $\delta^{13} \mathrm{C}$. However, these constraints are likely to be weak because of the uncertainties associated with gas exchanges and biological cycling.

In summary, the treatment of the Indian and Pacific oceans, the upper waters, and the formation of deep water as adjustable boundary conditions may result in the neglect of some constraints and thus in some overestimation of the uncertainties. However, we believe that this overestimation is slight and is largely compensated by the under-parameterization of other processes. 
The most obvious under-parameterization is the coarse resolution of the model. Conventional wisdom suggests that one needs a horizontal resolution better than $5^{\circ}$ to reproduce the ocean circulation in an even roughly realistic manner. Going from a $10^{\circ}$ horizontal resolution to $5^{\circ}$ would multiply the number of variables by four, but the number of measurements would remain roughly the same (a few data points have been lumped together to make them fit the $10^{\circ}$ grid; it would no longer be necessary to do so in a higher resolution grid and each data point would be taken into account separately so that the number of data points would slightly increase). So a higher resolution model would render the problem more undetermined. Similarly, we neglect the effect of the biological particle flux. This process is poorly known even in the modern ocean. Including it in the model would add further uncertainty. Finally, we treat $\delta^{13} \mathrm{C}$ as a tracer whereas in fact the ${ }^{13} \mathrm{C}$ concentration is the true tracer (see chapter II). Doing so is equivalent to assuming that the concentration of ${ }^{12} \mathrm{C}$ is a constant over the whole basin. A more rigorous approach would be to consider ${ }^{12} \mathrm{C}$ as another variable. This approach would probably not radically modify the ${ }^{13} \mathrm{C}$ balance but would introduce some uncertainty because there are no ${ }^{12} \mathrm{C}$ data for the LGM.

\section{Summary and concluding remarks}

Minor modifications of the modern North Atlantic circulation are sufficient to accommodate the LGM data and these modifications are also consistent with the South Atlantic data. Thus the modern ocean circulation is compatible with the paleo-data, in the context of a simple model of the ocean. However, circulations very different from the modern one are also possible, mostly because passive tracers like $\delta^{13} \mathrm{C}$ do not constrain the absolute fluxes, and only constrain the mass balance locally.

In the dynamical box model of the North Atlantic, Eastern Atlantic data do not constrain the strength of the DWBC because what happens in the interior of the ocean can be largely decoupled from the boundary current strength and thus from deep water formation rate. It might be possible to constrain the strength of NADW formation relative to AABW by looking at $\delta^{13} \mathrm{C}$ gradients close to the western boundary, as illustrated by a simple 2-box model. However, it is not clear 
whether a direct interpretation of the gradients is possible when circulation changes are combined with changes in preformed $\delta^{13} \mathrm{C}$ values.

In conclusion, there is no strong evidence from the paleo-tracers $\delta^{13} \mathrm{C}$ and $\delta^{18} \mathrm{O}$ for a reduced rate of formation of NADW during the LGM, and the modern ocean circulation appears to be consistent with these data. 


\section{Chapter VI - Summary and discussion.}

The distinction has been made throughout this work between water mass distributions and oceanic circulation.

Concerning the water mass distribution, inferred from $\delta^{13} \mathrm{C}$ data, it appears that the volume of $\mathrm{AABW}$ extended further north and that the volume of Lower NADW was reduced during the LGM, compared to the modern ocean. The extent of the difference between the glacial and the modern water mass distributions is not clear, however. One approach to quantifying these differences is to compare the position of the lines delimiting waters mostly composed of NADW and waters mostly composed of AABW in the glacial and in the modern oceans. This approach is inconclusive, however, because the $\mathrm{AABW}$ and the NADW $\delta^{13} \mathrm{C}$ end-members are poorly known in the glacial ocean.

An important point is that a change in the vertical distribution of $\delta^{13} \mathrm{C}$ at one given location does not necessarily indicate a change in the relative proportion of AABW and NADW. It may simply indicate a change in the composition of the end-members. More $\delta^{13} \mathrm{C}$ data are needed close to the regions of formation of deep water in order to better constrain the end-members.

The second part of this work concerns the interpretation of changes in the water mass distribution in terms of changes in the circulation of the deep ocean. In the paleoceanographic literature, no clear distinction is made between water mass distribution and ocean circulation. The reduced volume of Lower NADW during the LGM is generally interpreted as indicating a reduced rate of formation of deep water in the North Atlantic (Boyle and Keigwin, 1982; 1987; Duplessy et al., 1988). Using an inverse model of the Atlantic Ocean, it is shown in chapter V that, in fact, the interpretation of water mass distribution in terms of circulation is ambiguous.

Two almost identical circulations can maintain very different water mass distributions. There are only small differences between the modern ocean circulation, which results in the modern water mass distribution, and a glacial ocean circulation, which results in the LGM water mass distribution. The low $\delta^{13} \mathrm{C}$ values observed in the deep North Atlantic during the LGM are 
produced in the model simulation by a small northward flux of $\delta^{13} \mathrm{C}$ depleted waters across the equator; a significant reduction of the flux of Lower NADW is not required.

Two almost identical tracer distributions can be maintained by quite different oceanic circulations, one corresponding to a strong production of Lower NADW, and the other corresponding to a reduced production of Lower NADW. All that is required to maintain a given passive tracer distribution is that the flux divergence of this tracer vanishes in each box of the model. This condition does not suffice to constrain the absolute fluxes of water across the box interfaces; one can multiply all the water fluxes by any arbitrary constant, and support the same tracer distribution. Radioactive tracers, such as $\Delta^{14} \mathrm{C}$, are required to constrain the absolute fluxes of water. Passive tracers constrain the relative fluxes of water, but only locally. $\delta^{13} \mathrm{C}$ data in the Eastern Atlantic, for instance, constrain the relative fluxes of AABW and NADW in the Eastern Atlantic, but not the relative fluxes of AABW and NADW in the Western Atlantic. Most of the paleo-data come from the Eastern Atlantic, and thus provide only limited constraints on the ventilation of the Western Atlantic, where the strongest currents are expected (Stommel and Arons, 1960a\&b). Thus, transports in the DWBC are poorly constrained in the LGM Atlantic, and can vary over a wide range of values.

Therefore, it appears that the interpretation of water mass distribution, as evidenced by tracer data, in terms of circulation changes is ambiguous. Obtaining more $\delta^{13} \mathrm{C}$ data, or any other passive tracer data, will help to better define the water mass distribution, but will not remove the ambiguity of this interpretation.

The reduction of production of deep water in the North Atlantic during the LGM is a plausible scenario - a reduced flux of Lower NADW could presumably result in a smaller volume of NADW - but has yet to be confirmed by more conclusive data such as $\Delta^{14} \mathrm{C}$ data. GCMs show that the thermohaline circulation in the modern Atlantic contributes to the relatively warm climate of Northern Europe, and that a reduction in the rate of overturning in the Atlantic would result in a significant cooling of this continent (Rind et al., 1986). Thus, it could be argued on theoretical grounds that a reduction in the rate of formation of NADW is required to explain the cold climate of Europe during the Last Ice Age. Other explanations, however, are equally acceptable. The greater extent of continental glaciers and of sea-ice, for instance, resulted in an 
increased surface albedo during the LGM (Berger, 1988). Such an increased albedo must have been accompanied with reduced temperatures in the high latitudes of the northern hemisphere. This greater extent of the ice cover can be produced through a positive feedback mechanism, first proposed by Milankovitch (1941), which does not invoke a change in the oceanic heat transport. Schematically, the reduced high latitude summer insolation during glacial times, due to the periodic change in the earth's orbital parameter, permits an extension of the snow cover which results in an increase surface albedo, and subsequent cooling of the climate and extension of the snow cover. Thus a reduction in the oceanic heat flux is not needed to explain glacial-interglacial climatic changes in Northern Europe.

$\delta^{13} \mathrm{C}$ data are necessary to interpret $\Delta^{14} \mathrm{C}$ data, because of their ability to trace the various water masses. Indeed, the $\Delta^{14} \mathrm{C}$ signal in sea water is the sum of two contributions: the ageing of the water masses as they flow away from their sources, and the preformed values of $\Delta^{14} \mathrm{C}$ in the various water masses. The contribution of the preformed values of $\Delta^{14} \mathrm{C}$ can be removed using a passive tracer (Broecker et al., 1991) such as $\delta^{13} \mathrm{C}$. The remaining contribution, the ageing term, indicates the rate of ventilation of the deep ocean.

$\delta^{13} \mathrm{C}$ data, and $\delta^{18} \mathrm{O}$ data, can also be used to determine whether an oceanic circulation simulated by a forward numerical model is plausible, or not. Such a simulation has been attempted by Lautenschlager et al. (1992), using the General Circulation Model (GCM) of the Hamburg group. The $\delta^{13} \mathrm{C}$ distribution predicted by their model in the LGM ocean (Figure VI-1) is not consistent with the paleo-data. They obtain a $\delta^{13} \mathrm{C}$ minimum at about 500 meters depth, near the equator, in the Atlantic Ocean, which is not observed in any of the various reconstructions of the latitudinal distribution of $\delta^{13} \mathrm{C}$ (see chapter II). Lautenschlager et al. conclude that the circulation obtained by their model is inconsistent with the $\delta^{13} \mathrm{C}$ data, most probably because the fresh water fluxes at the air-sea interface, computed by an atmospheric GCM, are inadequate.

A possible application of $\delta^{13} \mathrm{C}$ data and $\delta^{18} \mathrm{O}$ data would be to assimilate them in a GCM similar to the one used by Lautenschlager et al. (1992). Using the adjoint method (Le Dimet and Talagrand, 1986), or other estimation methods, it would be possible to try to improve the estimate of the fresh water fluxes in order to make them consistent with the distribution of $\delta^{13} \mathrm{C}$ in the glacial ocean. Some significant technical difficulties would have to be overcome (see Tzipermann 

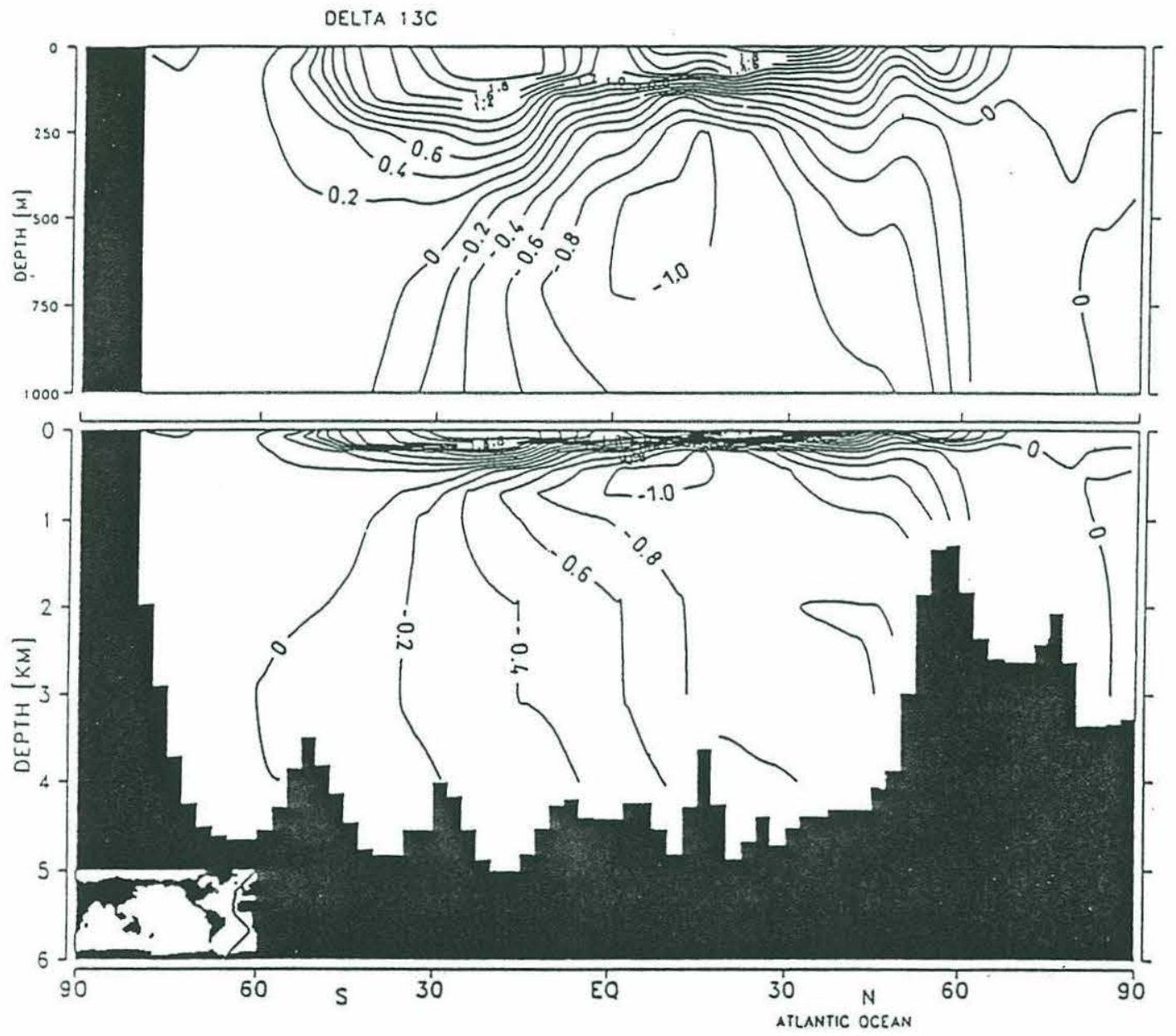

Figure VI-1: $\delta^{13} \mathrm{C}$ distribution predicted in the LGM Eastern Atlantic by an oceanic GCM. Contours in \%o. Figure from Lautenschlager et al. (1992). 
et al., 1992a\&b; Marotzke, 1992; for the problems encountered in modern ocean applications of the adjoint method). It would be worth the effort, however. As stated in the introduction of this thesis, one important objective of paleoceanography is to provide data in order to test climate models in circumstances very different from the present climate. Assimilating the paleo-data could confirm deficiencies in the fresh water fluxes estimated by atmospheric GCMs. It could also point toward some deficiencies in oceanic GCMs which have not been determined yet. Testing climate models with the Last Ice Age data has yet to be done. 


\section{Bibliography}

Anderson, L. A., and J. L. Sarmiento, 1994: Redfield ratios of remineralization determined by nutrient data analysis, Glob. Biogeochemic. Cycles, 8: 65-80.

Arakawa, A., and V. R. Lamb, 1977: Computational design of the basic dynamical processes of the UCLA general circulation model, in Methods in computational physics, J. Chang (eds.), 17, Academic Press, New York: pp. 173-265.

Bard, E., B. Hamelin, R. G. Fairbanks, and A. Zindler, 1990: Calibration of the ${ }^{14} \mathrm{C}$ time scale over the past 30,000 years using mass spectrometric U-Th ages from Barbados corals, Nature, 345: 405-409.

Barth, N., and C. Wunsch, 1990: Oceanographic experiment design by simulated annealing, J. Physic. Oceanogr., 20: 1249-1263.

Berger, A., 1988: Milankovitch theory and climate, Rev. of Geophysics, 26: 624-657.

Boyle, E. A., F. R. Schlater, and J. M. Edmond, 1976: On the marine geochemistry of cadmium, Nature, 263: 42-44.

Boyle, E. A., and L. D. Keigwin, 1982: Deep circulation of the North Atlantic over the last 200,000 years: geochemical evidence, Science, 218: 784-787.

Boyle, E. A., and L. D. Keigwin, 1987: North Atlantic thermohaline circulation during the past 20,000 years linked to high latitude surface temperature, Nature, 330: 35-40.

Boyle, E. A., 1990: Quaternary deepwater paleoceanography, Science, 249: 863-870.

Boyle, E. A., 1992: Cadmium and $\delta^{13} \mathrm{C}$ paleochemical ocean distributions during the stage 2 glacial maximum, Annu. Rev. Earth Planet. Sci., 20: 245-287.

Broecker, W. S., 1981: Geochemical tracers and ocean circulation, in Evolution of Physical Oceanography, B. A. Warren and C. Wunsch (eds.), the MIT Press, Cambridge, MA and London, England: pp. 434-460.

Broecker, W. S., and T.-H. Peng, 1982: Tracers in the sea, Eldigio Press, Palisades N.Y.: p. 690.

Broecker, W. S., D. Peteet, and D. Rind, 1985: Does the ocean-atmosphere system have more than one stable mode of operation?, Nature, 315: 21-26.

Broecker, W. S., T.-H. Peng, S. Trumbore, G. Bonani, and W. Wolfli, 1990: The distribution of radiocarbon in the glacial ocean, Glob. Biogeochem. Cycles, 4: 103-117. 
Bibliography

Broecker, W. S., W. M. Smethie, and G. Ostlund, 1991: Radiocarbon decay and oxygen utilization in the deep Atlantic Ocean, Glob. Biogeochem. Cycles, 5: 87-117.

Bruland, K. W., G. A. Knauer, and J. A. Martin, 1978: Cadmium in the northeast Pacific waters, Limno. Oceanogr., 23: 618-625.

Charles, C. D., and R. G. Fairbanks, 1992: Evidence from Southern Ocean sediments for the effect of North Atlantic deep-water flux on climate, Nature, 355: 416-419.

CLIMAP, 1976: Climate Long-range Investigation Mapping and Prediction: The surface of the ice-age earth, Science, 191: 1131-1137.

CLIMAP, 1981: Climate Long-range Investigation Mapping and Prediction, Leader A. McIntyre: Seasonal reconstructions of the earth's surface at the last glacial maximum, in Map and chart series 36 (text, maps, and microfiche), Geological Society of America.

Covey, C., 1988: Atmosphere and ocean heat transport: simulation versus observations, Climatic Change, 13: 149-159.

Curry, W. B., and G. P. Lohmann, 1985: Carbon deposition rates and deep water residence time in the equatorial Atlantic Ocean throughout the last 160,000 years, in The carbon cycle and atmospheric $\mathrm{CO}_{2}$ : Natural variations archean to present, E. Sundquist and W. S.

Broecker (eds.), Geophys. Monogr. Ser. 32, AGU, Washington D.C.: pp. 285-301.

Curry, W. B., and G. P. Lohmann, 1986: Late quaternary carbonate sedimentation at the Sierra Leone Rise (eastern equatorial Atlantic), Mar. Geol., 70: 223-250.

Curry, W. B., J.-C. Duplessy, L. D. Labeyrie, and N. J. Shackleton, 1988: Changes in the distribution of $\delta^{13} \mathrm{C}$ of deep water $\Sigma \mathrm{CO}_{2}$ between the last glaciation and the Holocene, Paleoceanography, 3: 317-341.

Curry, W. B., and G. P. Lohmann, 1990: Reconstructing past particle fluxes in the tropical Atlantic ocean, Paleoceanography, 5: 487-505.

Dansgaard, W., J. W. C. White, and S. J. Johnsen, 1989: The abrupt termination of the Younger Dryas climate event, Nature, 339: 532-534.

Duplessy, J.-C., N. J. Shackleton, R. G. Fairbanks, L. Labeyrie, D. Oppo, and N. Kallel, 1988: Deepwater source variations during the last climatic cycle and their impact on the global deepwater circulation, Paleoceanography, 3: 343-360.

Duplessy, J.-C., L. D. Labeyrie, A. Juillet-Leclerc, F. Maitre, J. Duprat, and M. 


\section{Bibliography}

Sarthein, 1991: Surface salinity reconstruction of the North Atlantic Ocean during the last glacial maximum, Oceanologica Acta, 14: 311-324.

Fairbanks, R. G., 1989: A 17,000-year glacio-eustatic sea level record: influence of glacial melting rates on the Younger Dryas event and deep-ocean circulation, Nature, 342: 637-642.

Fiadeiro, M. E., and G. Veronis, 1984: Obtaining velocities from tracer distributions, $J$. Phys. Oceanogr., 14: 1734-1746.

Fukumori, I., F. Martel, and C. Wunsch, 1991: The hydrography of the North Atlantic in the early 1980's. An atlas, Progress in Oceanography, 27: 1-100.

GEOSECS, 1980: sections and profiles, in GEOSECS Atlantic expedition, A. E. Bainbridge (eds.), 2, U.S. Government Printing Office, Washington D.C.: pp. 198.

Gill, P. E., M. H. Murray, and M. H. Wright, 1981: Practical optimization, Academic Press, London: p. 401.

Gill, A. E., 1982: Atmosphere-ocean dynamics, Academic Press, London: p. 662.

Hall, M. M., and H. L. Bryden, 1982: Direct estimates and mechanisms of ocean heat transport, Deep-Sea Res., 29: 339-359.

Hansen, J., and S. Lebedeff, 1987: Global trends of measured surface air temperature, $J$. Geophys. Res., 92: 13345-13372.

Hansen, J., and S. Lebedeff, 1988: Global surface air temperatures: update through 1987, Geophys. Res. Let., 15: 323-326.

IPCC, 1990: Climate Change, The Intergovernmental Panel on Climate Change scientific assessment, J. T. Houghton, G. J. Jenkins, J. J. Ephraums (eds.), University Press Cambridge, Cambridge U.K.: p. 365.

Johnsen, S. J., H. B. Clausen, W. Dansgaard, K. Fuhrer, N. Gundestrup, C. U. Hammer, P. Iversen, J. Jouzel, B. Stauffer, and J. P. Steffensen, 1992: Irregular glacial interstadials recorded in a new Greenland ice core, Nature, 359: 311-314.

Joussaume, S., R. Sadourny, and J. Jouzel, 1984: A general circulation model of water isotope cycles in the atmosphere, Nature, 311: 24-29.

Jouzel, J., N. I. Barkov, J. M. Barnola, M. Bender, J. Chappelaz, C. Genthon, V. M. Kotlyakov, V. Lipenkov, C. Lorius, J. R. Petit, D. Raynaud, G. Raisbeck, C. Ritz, T. Sowers, M. Stievenard, F. You, and P. You, 1993: 
Bibliography

Extending the Vostok ice core record of palaeoclimate to the penultimate glacial period, Nature, 364: 407-412.

Keffer, T., D. G. Martinson, and B. H. Corliss, 1988: The position of the Gulf Stream during quaternary glaciations, Science, 241: 440-442.

Keigwin, L. D., and E. A. Boyle, 1989: Late Quaternary paleochemistry of high-latitude surface waters, Palaeogeography Palaeoclimatology Palaeoecology, 73: 85-106.

Keir, R. S., 1988: On the late Pleistocene ocean geochemistry and circulation, Paleoceanography, 3: 413-446.

Kroopnick, P. M., 1985: The distribution of ${ }^{13} \mathrm{C}$ of $\Sigma \mathrm{CO}_{2}$ in the world oceans, Deep-Sea Res., 32: 57-84.

Kruger, J., 1993: Simulated annealing: a tool for data assimilation into an almost steady model state, J. Phys. Oceanogr., 23: 679-688.

Labeyrie, L. D., and J.-C. Duplessy, 1985: Changes in the ${ }^{13} \mathrm{C} /{ }^{12} \mathrm{C}$ ratio during the last 140000 years: high-latitude surface water records, Palaeogeography Palaeoclimatology

Palaeoecology, 50: 217-240.

Labeyrie, L. D., J.-C. Duplessy, J. Duprat, A. Juillet-Leclerc, J. Moyes, E. Michel, N. Kallel, and N. J. Shakleton, 1992: Changes in the vertical structure of the North Atlantic ocean between glacial and modern times, Quaternary Science Reviews, 11: 401-413.

Lautenschlager, M., U. Mikolajewicz, E. Maier-Reimer, and C. Heinze, 1992: Application of ocean models for the interpretation of atmospheric general circulation model experiments on the climate of the Last Glacial Maximum, Paleoceanography, 7: 769-782.

LeDimet, F. X., and O. Talagrand, 1986: Variational algorithms for analysis and assimilation of meteorological observations: theoritical aspects, Tellus, 38A: 97-110.

Lehman, S. J., and L. D. Keigwin, 1992: Sudden changes in north Atlantic circulation during the last deglaciation, Nature, 356: 757-762.

Levitus, S., 1982: Climatological atlas of the world ocean, US Government Printing Office, Washington, D.C.: p. 173.

Liebelt, P. B., 1967: An introduction to optimal estimation, Addison-Wesley, Reading, 


\section{Bibllography}

Mass.: p. 273.

Lohmann, G. P., and K. C. Lohmann, 1994: Vertical $\delta^{13} \mathrm{C}$ gradient in the South Atlantic during the Last Glacial Maximum recorded in benthic and planktonic foraminifera, article to be published in the proceedings of the symposium "The South Atlantic: present and past circulation", sponsored by the Oceanography Society, the World Ocean Circulacion Experiment, and the Scientific Committee on Oceanic Research.

Lutze, G. F., and H. Thiel, 1987: Cibicidoides wuellerstorfi and Planulina ariminensis, elevated epibenthic Foraminifera, in Beobachtungen an Benthos-Foraminiferen (Teilprojekt A3) Rep. Sonderforshungsbereich 313, A. V. Altenbach, G. F. Lutze and P. Weinhoiz (eds.), 6, Univ. Kiel, Kiel: pp. 17-30.

Mackensen, A., H.-W. Hubberten, T. Bickert, G. Fisher, and D. K. Fütterer, 1993: The $\delta^{13} \mathrm{C}$ in benthic foraminiferal tests of Fontbiota Wuellerstorfi (Schwager) relative to the $\delta^{13} \mathrm{C}$ of dissolved inorganic carbon in Southem Ocean deep water, Paleoceanography, 6: 587-610.

Manabe, S., and $\mathrm{K}$. Bryan, 1985: $\mathrm{CO}_{2}$-induced change in a coupled ocean-atmosphere model and its paleoclimatic implications, J. Geophysical Res., 90: 11689-11707.

Marotzke, J., 1992: The role of integration time in determining a steady state through data assimilation, J. Phys. Ocean., 22: 1556-1567.

Martel, F., and C. Wunsch, 1993: The North Atlantic circulation in the early 1980s-An estimate from inversion of a finite-difference model, J. Phys. Oceanogr., 23: 898-924.

Martin, J. H., R. M. Gordon, and S. E. Fitzwater, 1990: Iron in Antarctic waters, Nanure, 345: 156-158.

McCartney, M. S., S. L. Bennett, and M. E. Woodgate-Jones, 1991: Eastward flow through the Mid-Atlantic ridge ar $11^{\circ} \mathrm{N}$ and its influence on the abyss of the eastern basin, $J$. Physic. Oceanogr., 21: 1089-1121.

Mercier, H., 1986: Determining the general circulation of the ocean: a nonlinear inverse problem, J. Geophys. Res., 94: 5103-5109.

Mercier, $\mathrm{H}_{\text {. }}$ 1989: A study of the time-averaged circulation in the western North-Atlantic by simultaneous inversion of hydrographic and current meter data, Deep-Sea Res., 36: 297-313.

Mercier, H., M. Ollitrauit, and P.-Y. L. Traon, 1993: An inverse model of the North 


\section{Bibliography}

Atlantic general circulation using lagrangian float data, J. Phys. Ocean., 23: 689-715.

Milankovitch, M., 1941: Kanon der erdbestrahling, Canon of insolation and the ice-age problem, English translation by Israel Program for scientific translations, Jerusalem, 1969, $R$. Serbian Acad. spec. publ. 132, Sect. math. sci, 33:

Mix, A. C., 1987: The oxygen-isotope record of glaciation, in North America and adjacent oceans during the last deglaciation, W. F. Ruddiman and H. E. Wright (eds.), K-3, Geological Society of North America, Boulder, Colorado: pp. 111-135.

Nowlin, W. D., Jr., and J. M. Klinck, 1986: The physics of the Antarctic Circumpolar Current, Rev. of Geophysics, 24: 469-491.

Ochoa, J., and N. A. Bray, 1991: Water mass exchange in the gulf of Cadiz, Deep-Sea Res., 38: S465-S503.

Oppo, D. W., and S. J. Lehman, 1993: Mid-depth circulation of the subpolar North Atlantic during the last glacial maximum, Science, 259: 1148-1152.

Pedlosky, J., 1986: Geophysical fluid dynamics, second edition, Springer-Verlag, New York: p. 710.

Rind, D., D. Peteet, W. S. Broecker, A. McIntyre, and W. Ruddiman, 1986: W. Clim. Dynam., 1: 3-33.

Rintoul, S. R., and C. Wunsch, 1991: Mass, heat, oxygen and nutrient fluxes and budgets in the North Atlantic Ocean, Deep-Sea Res., 32: 57-84.

Roemmich, D., and C. Wunsch, 1985: Two transatlantic sections: meridional circulation and heat flux in the subtropical North Atlantic Ocean, Deep-Sea Res., 32: 619-664.

Sarthein, M., K. Winn, S. J. A. Jung, J.-C. Duplessy, L. Labeyrie, H. Erienkeuser, and G. Ganssen, 1994: Changes in east Atlantic deepwater circulation over the last 30,000 years: Eight time slice reconstructions, Paleoceanography, 9: 209-267.

Schlitzer, R., 1988: Modeling the nutrient and carbon cycles of the North Atantic, 1. Circulation, mixing coefficients, and heat fluxes, J. Geophys. Res., 93: 10699-10723.

Schlitzer, R, 1989: Modeling the nutrient and carbon cycles of the North Atlantic, 2. New production, particle fluxes, $\mathrm{CO}_{2}$ gas exchange, and the role of organic nutrients, J. Geophys.

Res., 94: 12781-12794. 


\section{Bibliography}

Schrag, D. P., and D. J. DePaolo, 1993: Determination of the $\delta^{18} \mathrm{O}$ of seawater in the deep ocean during the last glacial maximum, Paleoceanography, 8: 1-6.

Shackleton, N. J., 1974: Attainment of isotopic equilibrium between ocean water and the benthonic foraminiferal genus Uvigerina, isotopic changes in the ocean during the last glacial, Colloques Int. Centr. Nar. Rech. Sci., 219: 203-219.

Slowey, N. C., and W. B. Curry, 1992: Enhanced ventilation of the North Atlantic subtropical gyre thermocline during the last glaciation, Narure, 358: 665-668.

Stommel, H., and A. B. Arons, 1960a: On the abyssal circulation of the world ocean -I. Stationary planetary flow partems on a sphere, Deep-Sea Res., 6: 140-154.

Stommel, H., and A. B. Arons, 1960b: On the abyssal circulation of the world ocean -II. An idealized model of the circulation pattern and amplitude in oceanic basins, Deep-Sea Res., 6: 217-233.

Stuiver, M., and H. A. Polach, 1977: Discussion: reporting of ${ }^{14} \mathrm{C}$ data, Radiocarbon, 19: 355-363.

Stuiver, M., and H. G. Östlund, 1980: GEOSECS Atlantic radiocarbon, Radiocarbon, 22: $1-24$.

Talagrand, O., and P. Courtier, 1987: Variational assimilation of meteorological observations with the adjoint vorticity equation. I. Theory, Quat. J. Roy.Meteor. Soc., 113: 1311-1328.

Tarantola, A., and B. Valette, 1982a: Inverse problems = quest for information, $J$. Geophys., 50: 159-170.

Tarantola, A., and B. Valette, 1982b: Generalized nonlinear problems solved using the least-squares criterion, Rev. Geophys., 20: 219-232.

Tzipermann, E., and W. C. Thacker, 1989: An optimal control/adjoint equations approach to studying the oceanic general circulation, J. Phys. Oceanogr., 19: 1471-1485.

Tzipermann, E., W. C. Thacker, R. B. Long, and S. Hwang, 1992a: Oceanic data analysis using a general circulation model. Part I: simulations, J. Phys. Oceanogr., 22:

Tzipermann, E., W. C. Thacker, R. B. Long, S. Hwang, and S. Rintoul, 1992b: Oceanic data analysis using a general circulation model. Part II: A North Atlantic model, J. Phys. Oceanogr., 22: 1458-1485. 
Bibliography

Warren, B. A., 1981: Deep Circulation of the world ocean, in Evolution of Physical Oceanography, B. A. Warren and C. Wunsch (eds.), the MIT Press, Cambridge, MA and London, England: pp. 6-41.

Wunsch, C., 1977: Determining the general circulation of the oceans: a preliminary discussion, Science, 196: 871-875.

Wunsch, C., 1985: Can a tracer field be inverted for velocity?, J. Phys. Ocean., 15: 1521-1531.

Wunsch, C., 1990: Ocean data and ocean models, course notes.

Zahn, R., and A. C. Mix, 1991: Benthic foraminiferal $\delta^{18} \mathrm{O}$ in the ocean's temperature-salinity-density field: constraints on Ice Age thermohaline circulation, Paleoceanography, 6: 1-20. 
Appendix 1: Data compilation for the Last Ice Age, obtained from measurements in foraminifera shells (Cicidoides species only; the $\delta^{18} \mathrm{O}$ values are referenced to the Uvigerina species).

Columns 1 to 4: from left to right: name, latitude, longitude and depth of the core; Columns 5 and 6: LGM $\delta^{13} \mathrm{C}$ and LGM $\delta^{18} \mathrm{O}$ values in \%o;

Column 7: 1 indicates a core from the Eastern Atlantic, 0 indicates a core from the Western Atlantic;

Column 8: core-top $\delta^{13} \mathrm{C}$ value in \%o;

Columns 9 to 11: sources of the LGM $\delta^{13} \mathrm{C}$, LGM $\delta^{18} \mathrm{O}$, and core-top $\delta^{13} \mathrm{C}$ data; complete references indicated at the end of the appendix;

Column 12: comments. 


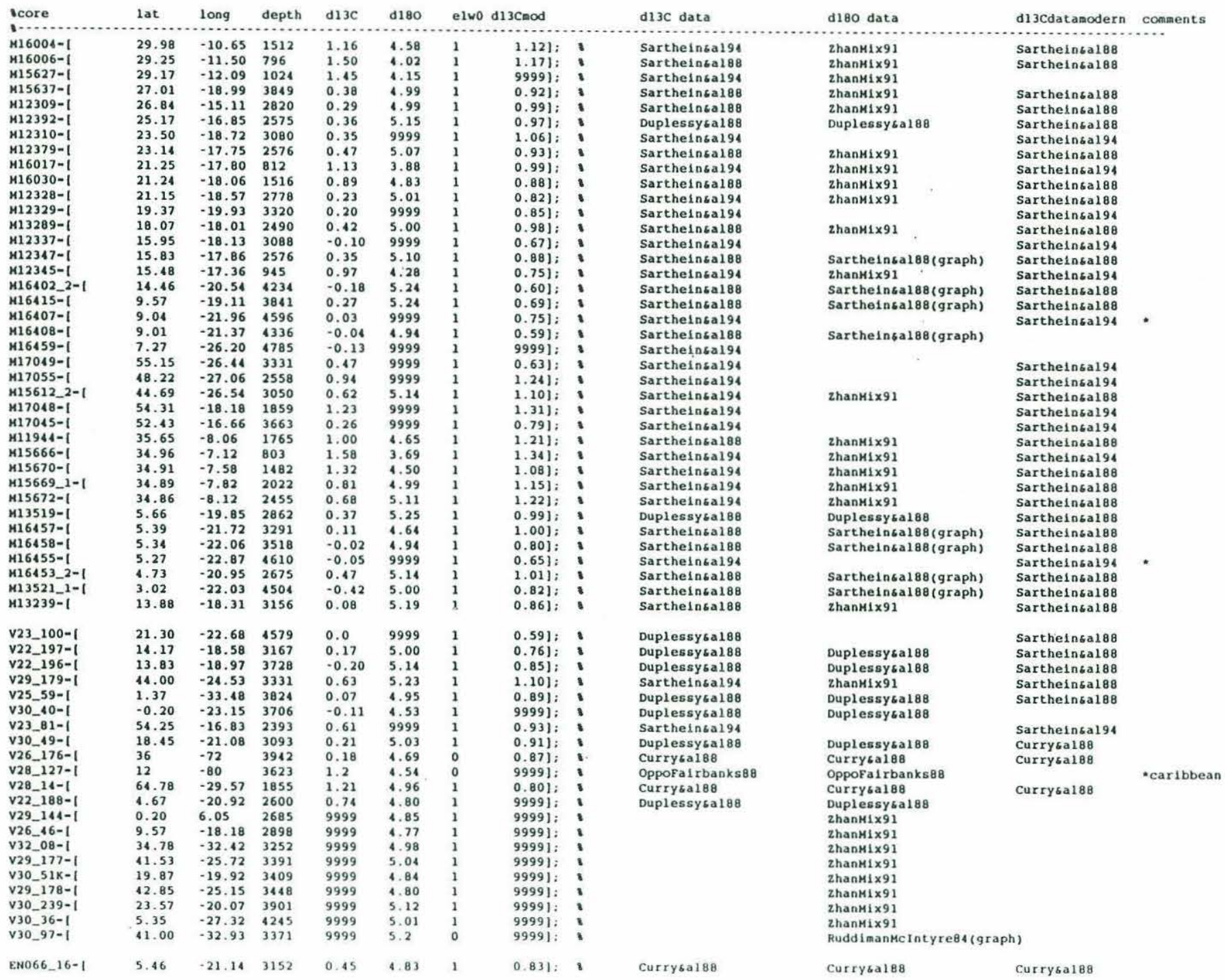




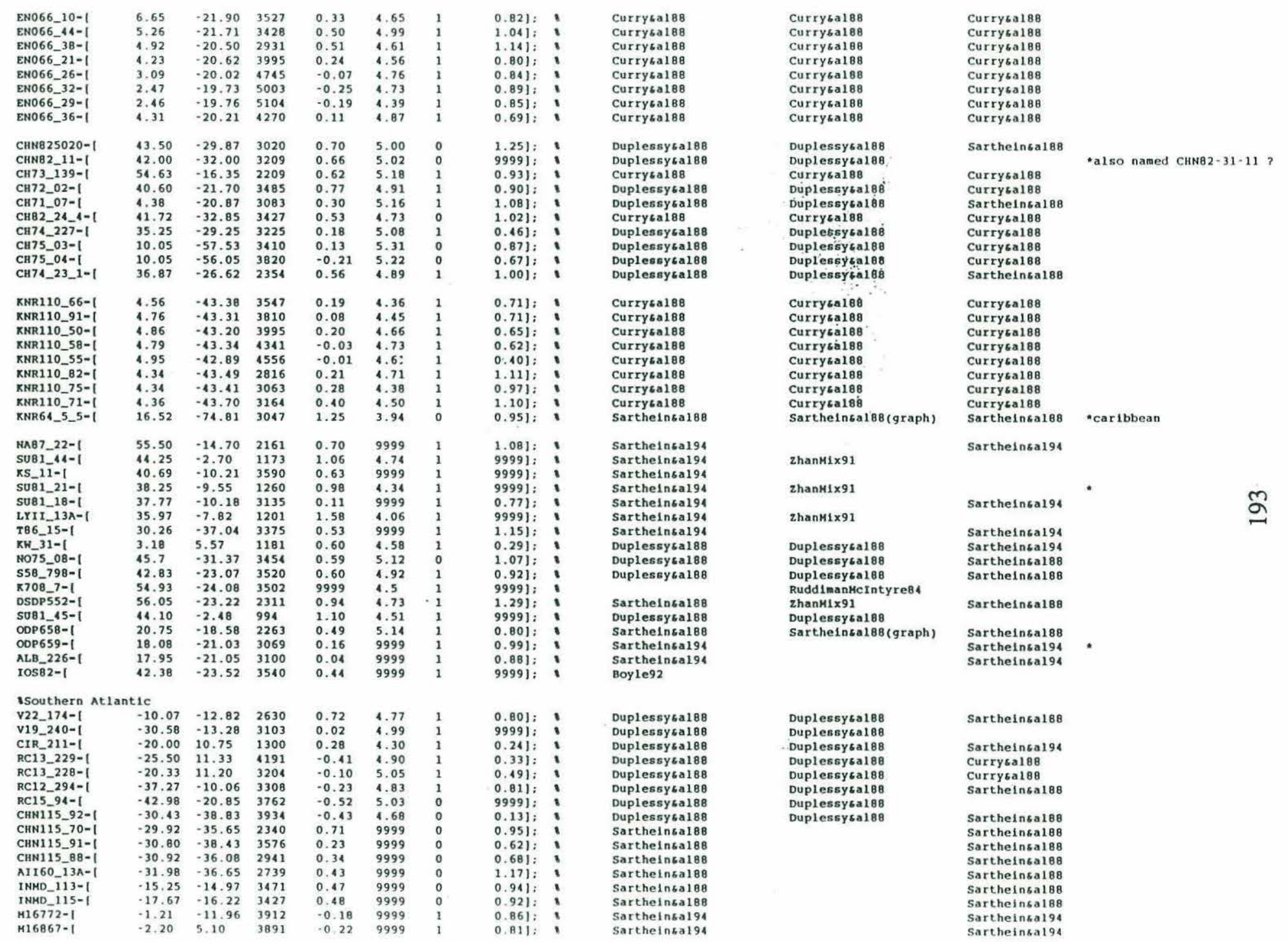




\begin{tabular}{|c|c|c|c|c|c|c|c|c|}
\hline $\begin{array}{l}\mathrm{BTT}_{\mathrm{N}-1}-1 \\
\mathrm{HG}_{2} 237\end{array}$ & $\begin{array}{l}-4.33 \\
-5.20\end{array}$ & $\begin{array}{l}10.43 \\
11.33\end{array}$ & $\begin{array}{l}1000 \\
1000\end{array}$ & $\begin{array}{l}0.24 \\
-0.10\end{array}$ & $\begin{array}{l}9999 \\
9999\end{array}$ & $\begin{array}{l}0.381: \\
-0.111:\end{array}$ & 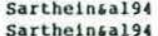 & $\begin{array}{l}\text { Curryba188 } \\
\text { Sartheinbal94 }\end{array}$ \\
\hline K1113-4-1 & -5.75 & -11.04 & 2374 & 0.45 & 9999 & $0.931 ;$ & Sarthelnbal94 & Sartheinsal94 \\
\hline RC11_83-1 & -41.30 & 14.48 & 4718 & -0.67 & 9999 & 99991: & Sarthe1nbal94 & \\
\hline
\end{tabular}

references:

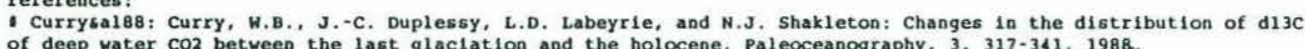
of deep water $\mathrm{CO}^{2}$ between the last glaciation and the holocene, Paleoceanography, $3,317-341,1988$.

- Sartheinaal88: Sarthein, K., K. HInn, J.C. Duplessy, and $K$. R. Fontugne: Global variations of surface ocean the last 21,000 years, paleoceanography, 3, 361-399, 1988 .

- Duplessyca188: Duplessy, J.C., N.J. Shackleton, R.G. Fairbanks, L.D. Labeyrie, D. Oppo, and N. Kal1e1: Deepwate Alobal deepwater circulation,

- ZhanH1x91: zahn, R., and A.C. M1x: Benthlc Foramin1feral d180, Paleoceanography, 6, 1-20, 1991.

- Sarthelncal96: Sarthe1n, H., K. Hinn, S.J.A. Jung, J-C Duplessy, L. Labeyrie, H. Erienkeuser, and G. Ganssen Changes in east At1antic deepwater c1rculation over the last 30,000 years: E1ght time silce reconstructions,

O Oppopalrbanks8: : Oppo, D.W.. and R.G. Falrbanks: Atlantic thermohaline c1rculation and atmospheric $\mathrm{CO}_{2}$. Paleoceanography, 5, p277-288. - Rudd1manKcIntyre84: Ruddiman, H.F., and $A$. HCIntyre: Ice-age theraal response and climat1c role of the surface Atlantic ocean, $40 \mathrm{~N}$ to $63 \mathrm{~N}$, Geological Soclety of America Bulletin, 95, 381-396, 1984.

- Shaketetonall18: Shakleton, N.J., and K.A. Hal1: Oxygen and carbon 1 sotope stat1graphy of Deep Sea pri111ng - Boyle92: Boyle, E.A: Cadmium and d13C paleochemical ocean distribution during the stage 2 glacial maximum, Annu, Rev. Planet. Sc1., 20, 245-87, 1992. 